\title{
Cultural group selection plays an essential role in explaining human cooperation: A sketch of the evidence
}

\author{
Peter Richerson \\ Department of Environmental Science and Policy, University of California- \\ Davis, Davis, CA 95616 \\ pjricherson@ucdavis.edu \\ www.des.ucdavis.edu/faculty/richerson/richerson.htm
}

\section{Ryan Baldini}

Graduate Group in Ecology, University of California-Davis, Davis, CA 95616 ryanbaldini@gmail.com https://sites.google.com/site/ryanbaldini/

\section{Adrian V. Bell \\ Department of Anthropology, University of Utah, Salt Lake City, UT 84112 av.bell@gmail.com http://adrianbell.wordpress.com/}

\section{Kathryn Demps}

Department of Anthropology, Boise State University, Boise, ID 83725 kathryndemps@boisestate.edu http://sspa.boisestate.edu/anthropology/faculty-and-staff/kathryndemps/

\section{Karl Frost}

Graduate Group in Ecology, University of California-Davis, Davis, CA 95616 kjfrost@ucdavis.edu https://sites.google.com/site/karljosephfrost/

\section{Vicken Hillis}

Department of Environmental Science and Policy, University of CaliforniaDavis, Davis, CA 95616

avhillis@ucdavis.edu http://vickenhillis.weebly.com

\section{Sarah Mathew}

School of Human Evolution and Social Change, Arizona State University, Tempe, AZ 85287

sarah.mathew@asu.eduｈttp://www.sarahmathew.net/Site/Home.htm

\author{
Emily K. Newton \\ Department of Psychology, Dominican University of California, San Rafael, CA \\ 94901 \\ emily.newton@dominican.edu http://emilyknewton.weebly.com/
}

\author{
Nicole Naar \\ Department of Anthropology, University of California-Davis, \\ Davis, CA 95616 \\ nanaar@ucdavis.edu

\section{Lesley Newson \\ Department of Environmental Science and Policy, University of California- Davis, Davis, CA 95616 \\ Ignewson@ucdavis.edu lesleynewson@gmail.edu https://www.researchgate.net/profile/Lesley_Newson/}

\section{Cody Ross}

Santa Fe Institute, Santa Fe, NM 87501

ctross@ucdavis.edu

http://scholar.google.com/citations?user=xSugEskAAAAJ

Paul E. Smaldino

Department of Anthropology, University of California-Davis, Davis, CA 95616 paul.smaldino@gmail.com http://www.smaldino.com/

\section{Timothy M. Waring}

School of Economics, University of Maine, Orono, ME 04469

timothy.waring@maine.edu http://timwaring.wordpress.com/

\section{Matthew Zefferman}

National Institute for Mathematical and Biological Synthesis, University of Tennessee, Knoxville, TN 37996

matt@zefferman.com http://www.zefferman.com/

\begin{abstract}
Human cooperation is highly unusual. We live in large groups composed mostly of non-relatives. Evolutionists have proposed a number of explanations for this pattern, including cultural group selection and extensions of more general processes such as reciprocity, kin selection, and multi-level selection acting on genes. Evolutionary processes are consilient; they affect several different empirical domains, such as patterns of behavior and the proximal drivers of that behavior. In this target article, we sketch the evidence from five domains that bear on the explanatory adequacy of cultural group selection and competing hypotheses to explain human cooperation. Does cultural transmission constitute an inheritance system that can evolve in a Darwinian fashion? Are the norms that underpin institutions among the cultural traits so transmitted? Do we observe sufficient variation at the level of groups of considerable size for group selection to be a plausible process? Do human groups compete, and do success and failure in competition depend upon cultural variation? Do we observe adaptations for cooperation in humans that most plausibly arose by cultural group selection? If the answer to one of these questions is "no," then we must look to other hypotheses. We present evidence, including quantitative evidence, that the answer to all of the questions is "yes" and argue that we must take the cultural group selection hypothesis seriously. If culturally transmitted systems of rules (institutions) that limit individual deviance organize cooperation in human societies, then it is not clear that any extant alternative to cultural group selection can be a complete explanation.
\end{abstract}

Keywords: competition; culture; evolution; group selection; heritable variation; institutions; norms 
Peter Richerson is Distinguished Professor Emeritus in the Department of Environmental Science and Policy at the University of California, Davis. He was trained as an entomologist and aquatic ecologist in the 1960s and acquired an interest in cultural evolution through teaching an interdisciplinary course on human ecology in the early 1970s. His work includes two books: Culture and the Evolutionary Process (University of Chicago Press, 1985) and Not By Genes Alone: How Culture Transformed Human Evolution (University of Chicago Press, 2005), coauthored with Robert Boyd.

Ryan BALDINI is an alumnus of the Graduate Group in Ecology at the University of California, Davis. His research focuses on human behavioral evolution, geneculture coevolution, and life history theory. He is currently using mathematical models to study rapid life history evolution in historical human populations.

ADrian V. Bell is Assistant Professor of Anthropology at the University of Utah. He is primarily interested in the cause and consequences of migration on human social evolution. Using theoretical and empirical approaches motivated by cultural evolutionary theory, he conducts fieldwork among the Tongan people of the South Pacific and migrant communities in the United States.

Kathryn Demps is an Assistant Professor of Anthropology at Boise State University. She specializes in human behavioral ecology, particularly resource extraction. She is interested in how people learn about and exploit local environments and how these behaviors change over time.

KARL Frost is a Ph.D. candidate in Ecology at the University of California, Davis. He researches the cultural evolution of prosociality via religion and ritual practices, using behavioral experiments, gene-culture coevolution models, and field research in Canada looking at environmental activism in the face of the tar sands oil industry and an antagonistic government. He also directs the Body Research Physical Theater and is interested in cross-cultural exchange of theater practice as theater anthropology and arts-science fusion.

VICKen HiLlis is a Postdoctoral Researcher in the Department of Environmental Science and Policy at the University of California, Davis. He received his Ph.D. in Ecology (emphasis on Environmental Policy and Human Ecology) at UC Davis. His research applies cultural evolutionary theory to environmental behavior and decision-making. He has examined agricultural sustainability in California and intergenerational cooperation using behavioral experiments in the lab. His work, combining theoretical, experimental, and fieldbased approaches, has been published in the Proceedings of the National Academy of Sciences, USA (PNAS), Ecology and Society, and other journals.

Sarah Mathew is an Assistant Professor in the School of Human Evolution and Social Change at Arizona State University. She studies the evolution of human cooperation and conducts fieldwork among the Turkana, an egalitarian pastoral group in northern Kenya, examining how they solve the collective action problem in largescale warfare. She currently is spearheading a threeyear field study among the Turkana, examining the evolutionary foundations of cooperation and moral judgments.

Emily K. Newton is an Assistant Professor of Psychology at the Dominican University of California. She studies the influence of social learning on the development of psychological understanding and prosocial behavior in young children. Her work has been published in Developmental Psychology and Infancy.

Nicole NaAr is a Ph.D. candidate in Anthropology at the University of California, Davis. Her research focuses on how community diversity affects fisheries management in Mexico, using theory and methods from cultural evolution, human behavioral ecology, and political ecology.

Lesley Newson is a Research Associate in the Department of Environmental Science and Policy at the University of California, Davis. She is interested in the continuing evolution of values that occurs after populations restructure and its members develop wider and shallower social networks.

Cody Ross is a Postdoctoral Fellow at the Santa Fe Institute and is the author of several publications in the area of molecular anthropology, gene-cultural coevolution, and human behavioral ecology. He is currently working on projects related to the inter-generational transmission of relational, embodied, and material wealth.

Paul E. Smaldino is a Postdoctoral Scholar in the Department of Anthropology at the University of California, Davis. His research focuses on how organizational patterns emerge in humans and other social organisms, with perspectives drawn from psychology, evolutionary ecology, and the social sciences. He is particularly interested in the use of computer simulations to uncover principles of social dynamics and evolution. He is the author of a previous BBS target article, "The cultural evolution of emergent group-level traits," published in 2014.

Timothy M. Waring is an Assistant Professor of socialecological systems modeling at the School of Economics, University of Maine. He studies the evolution of human culture and cooperation in environmental dilemmas. He leads a national network on evolutionary approaches to sustainability, funded through two working groups at the National Institute for Mathematical and Biological Synthesis and the National Socio-Environmental Synthesis Center. He was awarded a National Science Foundation CAREER grant for his research on the evolution of local food organizations in 2014.

Matthew Zefferman (né Zimmerman) is a Postdoctoral Research Fellow at the National Institute for Mathematical and Biological Synthesis, USA. His research focuses on cultural evolution and the origins of large-scale political institutions. 
Richerson et al.: Cultural group selection plays an essential role in explaining human cooperation

A tribe including many members who, from possessing in a high degree the spirit of patriotism, fidelity, obedience, courage, and sympathy, were always ready to aid one another, and to sacrifice themselves for the common good, would be victorious over most other tribes; and this would be natural selection.

— Charles Darwin (1874, pp. 178-79)

\section{Introduction}

Few scholars doubt that the scale of cooperation found in contemporary human societies is unusual. We typically live in large, rule-bound, anonymous societies in which the mean genetic relatedness between members is low (Moffett 2013). Ethnographic evidence from hunter-gatherer societies suggests that our Late Pleistocene ancestors already had many interactions with non-kin that were regulated by systems of norms and institutions, even in societies numbering only a few hundred to a few thousand (Hill et al. 2014). If that is the case, then there are two key evolutionary questions: (1) How did cooperation among nonkin evolve in small-scale societies in prehistoric times, and (2) how did small-scale societies evolve into much larger and more complex societies during the Holocene? We outline here the evidence that supports the hypothesis that cultural group selection (CGS) was and still is an important force in the evolution of human societies. We compare CGS to other evolutionary mechanisms that have been proposed to account for human sociality. See Boehm (2012), Bowles and Gintis (2011), and Cronk and Leech (2013) for reviews of the various mechanisms proposed to date.

Heretofore, the debate about the importance of CGS relative to competing explanations for human cooperation has not been fully engaged. Some debates have focused on narrow issues such as the interpretation of experimental results (Hagen \& Hammerstein 2006) and the assumptions of mathematical models (Boyd et al. 2011; Lehmann et al. 2008). Reviews of evolutionarily plausible accounts of human cooperation often do not discuss CGS (e.g., Sigmund 2007) or treat it as an unsupported conjecture or semantic error (e.g., West et al. 2007). Even the sympathetic commentator, E. A. Smith (2010), remarks that CGS is under-theorized and in need of much more empirical analysis. We provide a guide to the relevant theory and argue that the empirical evidence from a range of domains provides robust qualitative support for the point that CGS describes real and important processes in human social evolution. In many domains, the best evidence is quantitative. We cannot provide a precise quantitative account of the importance of CGS compared to competing (but generally not mutually exclusive) processes in the evolution of human cooperation. That is an ongoing project with recent pioneering studies in critical areas. We will discuss the following hypotheses:

1. Cultural group selection. The CGS hypothesis is an updated version of the one that Darwin (1874) formulated in The Descent of Man, the essentials of which are quoted in our epigraph (Richerson \& Boyd 2010). Darwin lacked the modern distinction between genes and culture, but in Descent he argued that the organic differences between human groups were small, while the differences in durable cultural features-customs and traditions were the terms he used-were large. He also noted that human groups - he uses the term tribe in the quote in our epigraph-compete. If this is the case, then group selection on cultural variation is an important force. We sketch the CGS hypothesis in more detail in section 2.

2. Culture doesn't evolve in the Darwinian sense. One critique of cultural evolutionary hypotheses generally is that transmitted culture, if it exists, lacks the properties to evolve in a Darwinian fashion at all, much less at the group level (Brown \& Richerson 2014; Pinker 2012; Tooby 2014; Tooby \& Cosmides 1992). The objection of some evolutionary biologists to CGS (e.g., West et al. 2011) seems to turn on a similar argument. If culture is taken to be a proximate mechanism rather than an ultimate cause, and if it is true that proximate mechanisms cannot influence the ultimate evolutionary processes, then culture cannot play any active role in the evolutionary process. For a general response to this argument, see Laland et al. (2011).

3. Kin selection and reciprocity suffice. Many alternatives to CGS argue that the familiar evolutionary processes acting on genes-reciprocity and inclusive fitness - which generally explain cooperation in nonhumans, are also sufficient to explain the case of human cooperation (e.g., Alexander 1987). Henrich et al. (2003) provide a useful discussion of the most prominent of these. The issue here is not whether effects such as nepotism, reputation building, and other mechanisms of cooperation supported by reciprocity and inclusive fitness exist - no one doubts this - but whether they are sufficient to explain the large-scale cooperation in human societies. Some hypotheses hold that CGS became important fairly late in human evolution and that a considerable amount of cooperation evolved earlier due to kin selection and reciprocity (Tomasello et al. 2012). Campbell (1975) suggested that CGS only became important once state-level societies developed, about 5,000 years ago. In simpler societies at least, individuals might bargain and negotiate their way to efficient social equilibria, as some game theorists argue (see Bowles \& Gintis 2011, Ch. 5 for a critical review).

4. Pleistocene social mechanisms mismatched in Holocene environments. A related alternative hypothesis holds that our innate social psychology evolved in the Pleistocene in societies that were so small that the familiar mechanisms mentioned previously could entirely account for their cooperative aspects. Subsequently, in the radically different environments of the Holocene, the cognitive mechanisms that evolved in the Pleistocene often caused people to behave in novel ways in environments that were outside the range of those in which the mechanism evolved. Some evolutionary psychologists argue that large-scale cooperation may be a by-product of a Pleistocene cognitive mechanism expressed in much larger, more anonymous Holocene societies (Delton et al. 2011; Krasnow et al. 2012; Tooby \& Cosmides 1989; but see Zefferman 2014a).

5. A role for genetic group selection. Some alternate theories invoke genetic group selection (on groups larger than those composed of close kin) as at least a partial explanation for human sociality (Rushton 1989; D. S. Wilson 2002; E. O. Wilson 2012). Several authors have imagined hybrid models in which culturally transmitted institutions reduce within-group phenotypic variation to such low 
Richerson et al.: Cultural group selection plays an essential role in explaining human cooperation

levels that modest amounts of between-group genetic variation can come under selection (Boehm 1997; Bowles \& Gintis 2011, Ch. 7; D.S. Wilson \& Kniffin 1999). We propose that CGS can exert selection on genes via culturally transmitted cost and benefit schedules (coevolutionary social selection). Culture-led gene-culture coevolution could produce much the same result as group selection more directly on genes.

6. Trigger hypotheses. Many hypotheses regarding human origins invoke some key selection event deep in our evolutionary history that set us on a path that led to modern human culture and societies. Proposals of this sort include: bipedality (Washburn 1959); intelligence (Pinker 2010); cooperative breeding (Burkart et al. 2009); cooperative hunting (Stanford 1999), language (Smith 2010); cooking (Wrangham 2009); and docility (Simon 1990). Such hypotheses are difficult to test independently of the coevolutionary extension of the CGS hypothesis. In living hunter-gatherers, and other modern humans, culture is closely involved in all of these phenomena (Hill et al. 2009). The paleoanthropological record is remarkably hard to interpret with regard to the order in which the various components of modern human sociality evolved and which components coevolved. Bipedal locomotion may have been necessary, but it was not a sufficient trigger because bipedality evolved in the Australopithecine lineage well before the record contains evidence of any advance in cultural behavior over that in nonhuman apes. On the other hand, even the most innatist theories of language evolution imagine a culture-led gene-culture coevolutionary process driving language evolution (Pinker \& Bloom 1990). Thus, we have little to say about hypotheses of this type.

In sum, if CGS is an active process in human social evolution, then we should see evidence that: (1) culture acts as an inheritance system; (2) group-variable culturally transmitted social norms and institutions are among the domains of culture so transmitted; and (3) success or failure in intergroup competition is frequently caused by institutional variation. As a result of these three features, we should also find evidence of: (4) group-level functionality in cultures, particularly in their social norms and institutions. The CGS hypothesis is thus vulnerable to disproof in several empirical domains. Competing hypotheses argue that cultural systems lack one or more of these features, or possess them to such a limited extent that the explanation for human sociality must depend in the main on other processes.

\section{The cultural group selection hypothesis}

In this section, we characterize the CGS hypothesis. Three distinct CGS processes exist, united by being driven by group-level heritable cultural variation, but differing in the mechanism of selection. Two of these forms are driven by human choices - choices in part based on prosocial emotions like Darwin's sympathy and patriotism. Because prosocial impulses play such a large role in CGS, it is important to account for their evolution. In the second part of this section, we describe the "tribal social instincts hypothesis," a modernization of Darwin's proposal, which can, in principle, explain the evolution of prosocial emotions.
Humans exhibit a wide variety of culturally transmitted organizational phenomena larger than, and different from, those reflecting biological relatedness and close pairwise partnerships. These include corporate kin groups, ethnicities, nations, classes, castes, religions, clubs, nations, and empires. CGS is potentially acting on more than one of these groups simultaneously, leading many authors to speak of multi-level selection. The most ancient groups are the ethnolinguistic tribes into which simpler societies are generally divided. As we discuss below, selection on tribes can account for the innate foundation of our social psychology via the process of gene-culture coevolution. The evolution of complex societies in the last five millennia has generated a diverse array of social groups that can vary culturally, compete, and be subject to group selection. We discuss business firms and religions as examples. While modern nations and the subgroups of which they are composed differ in many ways from ancestral tribes, they rest on the innate foundations that evolved from competition between ethnolinguistic units (Richerson \& Boyd 1999). Given their short history, selection on modern groups will not have had a major impact on genes, but we argue that it has been very important in the ongoing evolution of complex societies.

\subsection{How human culture facilitates CGS}

How do cultures generate the group-level variation that CGS requires, and what sort of selective processes act on that variation? In this section, we define the CGS hypothesis by reviewing the mechanisms that make cultural inheritance and cultural evolution quite different from genetic evolution.

The mechanisms that maintain intergroup variation are the following:

1. Accurate, rapid social learning. Human cognition includes adaptations for imitation and teaching that allow the cumulative evolution of complex traits, including norms and institutions that regulate social behavior (Tomasello 1999). See section 3.

2. Conformist social learning. If people tend to differentially copy locally common behaviors, the homogenizing effect of migration will be reduced and group differences will be maintained (Boyd \& Richerson 1985).

3. Coordination payoffs. If the payoff to any strategy depends on the local frequency of its use, then even subpopulations in identical environments may reach different stable equilibria over time. Frequency-dependent payoffs and multiple stable equilibria are probably very common in human social institutions (Cooper 1999).

4. Punishment of deviant behaviors. Punishment of those who engage in uncommon behaviors can stabilize essentially any norm within a group, regardless of its other adaptive consequences (Boyd \& Richerson 1992b). Since these norms will often be influenced by historical happenstance, groups will often end up with different sets of morally enforced customs. Punishment is a strong force if the punishing is cheap to the punishers but costly to the punished.

5. Strong prestige bias or one-to-many transmission. If people learn disproportionately from those who are most prestigious or successful in their local population, then the "effective size" of the cultural population may be 
Richerson et al.: Cultural group selection plays an essential role in explaining human cooperation

small and cultural drift will diversify populations (CavalliSforza \& Feldman 1981).

6. Symbolic markers of group boundaries. As groups differentiate, the importance of learning the locally proper behaviors and customs becomes increasingly important. If neutral marker traits correlate with behavioral norms that are difficult to observe, then selection may favor attentiveness to these markers as reliable signals of whom to imitate (McElreath et al. 2003). Dialect is an example (see sect. 4.2). This contributes to group differentiation by facilitating in-group assortment and reducing the chances that migrants are imitated, especially in those regions where neighboring groups interact.

7. Institutional complexity that generates inertia and reduces borrowing. Institutions are often a complex array of norms organized to solve cooperation dilemmas and navigate the intricacies of coordination (see sect. 4.3). The functioning of complex institutions is highly dependent on tacit knowledge (Aoki 2001). Even in-group members may have an incomplete explicit understanding of how an institution works, and the overall rules driving the institution may be distributed among the norms of various specialized participants. This makes the institution quite opaque to out-groups, limiting the diffusion of institutions between societies.

The high group-level variation between human groups sets the stage for the evolution of group-beneficial behaviors. There are three distinct processes by which groupbeneficial behavior can spread:

1. Natural selection. Groups exhibit variation in behaviors that affect the rate at which they grow, produce daughter groups, overcome resource constraints, avoid internal political conflict, succeed in war, and ultimately replace other groups. This provides a relatively slow form of selection for traits that contribute to group success (Soltis et al. 1995).

2. Selective imitation of successful groups. If people prefer to copy successful individuals, and if they have some contact with out-group members, then members of less successful groups will sometimes copy members of more successful groups. This favors the spread of group-beneficial norms and institutions (Boyd \& Richerson 2002). This force may be especially powerful if the new cultural characteristic is adopted by particularly influential group members who are more likely to be copied by others in their group.

3. Selective migration between groups. Individuals in dysfunctional groups may migrate to another group if they observe that the other group more successfully maintains behaviors that benefit all members (Boyd \& Richerson 2009). The resulting diminution of unsuccessful groups may weaken their competitive ability and/or encourage leaders to adopt successful foreign norms, strengthening the two processes defined previously.

All three selective processes will tend to reduce grouplevel variation. Thus, the standing group-level variation is a balance between variation-creating and variation-reducing forces. It is an empirical matter whether cultural evolutionary processes can maintain substantial between-group variation or whether, as is the case with genetic variation, appreciable group variation is hard to maintain. See section 4.1, particularly for a quantitative discussion of this issue.
Throughout the remainder of this article, we will generally use economic terms, such as "cost," "benefit," and "success," to characterize the contribution of traits to CGS. Evolutionary biologists generally express such terms in the currency of genetic fitness. Cultural evolution and gene-culture coevolution are considerably more complex. A cultural variant that attracts many imitators has a reproductive success which is not necessarily tied to biological reproduction. For example, prosocial leaders gain prestige, which may attract imitators not related genetically to the leader. In the case of the three mechanisms of CGS and the mechanism of gene-culture coevolution, we can use models to specify the costs, benefits, and success of cultural variants in terms of changes in their frequency. Model-fitting approaches have been used to estimate the strength of complex evolutionary forces in laboratory cultural evolution experiments (McElreath et al. 2008). So far, too few quantitative studies have been performed on CGS and competing processes to allow for much but qualitative judgments. One of our purposes here is to make the case that qualitative investigations already performed amply support the pursuit of more definitive quantitative data. We will discuss the relatively small number of pioneering quantitative studies of cultural evolution that have been conducted to date (see, e.g., our Figure 1 in section 4.1 of this article). This work has demonstrated the feasibility of quantitative work on every phase of cultural evolution. For example, ambitious efforts are underway to use historical and archaeological data to construct synthetic time series that will be suitable for quantitative model fitting investigations (Collard et al. 2010; Turchin 2009; Turchin et al. 2012).

Note that many cultural evolutionary processes generate cultural variation and cause evolution. CGS is only relevant to situations in which groups compete; it is not a complete theory of the evolution of cultural variation.

\subsection{The tribal social instincts hypothesis}

If the three CGS processes described in the previous section have operated over spans of time consistent with the evolution of genes, then culture-led gene-culture coevolution may have favored genes that encourage group advantageous behavior.

How did human culture become evolvable at the group level? Richerson and Boyd (1998) proposed a "tribal social instincts" hypothesis to account for the emergence of innate social predispositions that support the normand institution-bound social life of humans. Once humans could culturally transmit elementary norms, simple moral communities would exist. Moral norms that evolved under the influence of CGS would exert social selection on human genes via mechanisms such as selective child neglect, mate choice, and punishment (Feldman \& Laland 1996; Newson 2013). Perhaps the earliest cultural norms merely solidified the bonds of kinship and reciprocity that were evolving through participation in systems of cooperative breeding (Chapais 2008; Hrdy 2009). Infants younger than one year prefer puppets that are helpful to others over those that are antisocial (Hamlin et al. 2011), suggesting that our social predispositions are partly innate. See section 4.2 for more evidence on this point.

The complexity of technology that a group can maintain is a function of its size (Henrich 2004; Shennan 2001). 
Richerson et al.: Cultural group selection plays an essential role in explaining human cooperation

Docile individuals more inclined to conform to norms would find it easier to enter larger more norm-bound groups and hence be able to acquire more sophisticated technology (Simon 1990). A population of more docile individuals could then permit the cultural evolution of slightly more sophisticated and effective norms. This would allow groups to maintain more reliable cooperation and tools, which would, in turn, be favored by CGS acting on this variation.

A recent quantitative estimate of rates of cultural evolution using a large body of historical and archaeological data showed that it evolves considerably faster than genes (Perreault 2012). Cultural evolution will thus tend to be the leading process, and genetic evolution the lagging process, in gene-culture coevolution. Over repeated rounds of such coevolution, stretching back perhaps several hundred thousand years, our Pleistocene ancestors came to have institutionalized social systems with (if ethnographic analogs are reliable) complex sets of linked norms organizing tribal societies of a few hundred to a few thousand people (Hill et al. 2014). At some point, language became sufficiently rich that complex norms could be explained to children, and participants could make promises, negotiate conflicts, and discuss institutional innovations (see sect. 6.1).

Several hypotheses compete with this coevolutionary account. Ayala (2010) argues that natural selection acted directly on human cognitive capacities to the point that all of the innate underpinnings necessary for the maintenance of norms and institutions were in place before the cultural transmission of them was important. Other accounts root human sociality in genetically evolved skills for social exchange, with no important role for cultural evolution (Tooby \& Cosmides 1992).

Evidence currently exists that supports the hypothesis of culture-led gene-culture coevolution for a few simple genetic traits whose function is well known, but unfortunately not yet for genes related to behavior. The best understood examples of culture-led gene-culture coevolution are adult lactase persistence (Durham 1991) and the hemoglobin polymorphisms that confer resistance to malaria (Allison 1964). Recent molecular evidence suggests that the genes associated with these and other phenotypic changes swept to appreciable frequencies in the Holocene, as agricultural diets exerted selective pressures on digestive physiology (Itan et al. 2009; Tishkoff et al. 2007) and human populations became dense enough to carry epidemic diseases (Wolfe et al. 2007). The dramatic changes of modern environments are resulting in detectable selection on genes related to health and fertility (Byars et al. 2010; Kohler et al. 1999). Genome-wide scans for genes under selection and comparative studies on chimpanzee-human differences detect strong recent selection at a large number of loci affecting genes that are expressed in the nervous system and in hormone producing glands. These are candidates to test the tribal social instincts hypothesis and its competitors, but the specific functions of the selected alleles are as yet unknown (Laland et al. 2010; Ross \& Richerson 2014). For example, if genes for docility were selected early in the hominin lineage before we have evidence for sophisticated culture, they are less likely to have been a product of culture-led gene-culture coevolution than if they evolved in the last 150,000 years as culture increased to modern levels of sophistication.
Several possible cases of genes related to behavior coming under culture-induced selection in the Holocene have been proposed. Cochran and Harpending (2009) suggest that the rise of modern urban occupations have favored genes for higher intelligence in some populations. Chiao and Blizinsky (2010) argue that neural processing of social status cues differs between more and less hierarchical societies. The genetic mechanism proposed as a cause of such differences is variation in a serotonin transporter gene.

Genomic tools promise to substantially improve our ability to test the tribal social instincts hypothesis by allowing us to estimate the dates at which major selective events occurred (Richerson et al. 2010). Ongoing advances in whole genome sequencing will provide much larger and higher resolution samples of genomes. Improvements in statistical and modeling techniques promise to improve the resolution for detecting signatures of selection, and for discovering their selective consequences more easily (Pritchard et al. 2010). Over the next decade, we can expect a flood of new data that can be used to test hypotheses related to gene-culture coevolution.

\section{Culture acts as an inheritance system}

Two quite distinct lines of evidence show that human culture acts as an inheritance system. First, evidence from developmental studies shows how a cognitive system for imitation arises in the first 12 months of life and dovetails with adult behaviors that support children's social learning. Other apes have a much more rudimentary social learning system. Second, historical evidence shows that human culture exhibits a phylogenetic pattern of descent with modification in which ancestral commonalities can be traced back thousands of years. Together, these lines of evidence cast strong doubt on the idea that most human behavioral differences have arisen via an extreme version of the "evoked culture" hypothesis (Tooby \& Cosmides 1992). By "evoked culture," these authors mean that human minds have a universal psychological architecture, selected in Pleistocene environments, which allows for the expression of phenotypic differences during development in various evolutionarily relevant domains such as social exchange and mate choice. It is also difficult to see how the developmental and historical evidence can be reconciled with an extreme version of the later improvisational intelligence hypothesis of Cosmides and Tooby (2001). Individual humans might invent complex adaptations on the spot as needed, with little or no need to depend on cultural inherited traditions. If this were so, children would have little need to acquire complex adaptations with a system adapted for high-fidelity imitation. Nor would we observe deep phylogenetic patterns in comparative and historical data; high rates of individual improvisational innovation would rapidly erase any historical signal due to culture transmission by accurate imitation. This is not to defend a blank slate hypothesis. Gene-based cognitive constraints on cultural evolution are surely many. Nor do we mean to imply that humans are not highly intelligent. Nor are we even sure that anyone defends extreme versions of the evoked culture or improvisational intelligence hypotheses. It is easy to imagine that a phenomenon like religion is co-produced by genetically inherited cognitive biases and 
Richerson et al:: Cultural group selection plays an essential role in explaining human cooperation

the cultural transmission of specific religious doctrines (Gervais et al. 2011), and the great speed of cultural relative to genetic evolution no doubt owes much to our capacity for foresightful innovation (Perreault 2012).

\subsection{A cognitive system for accurate, voluminous cultural transmission}

Children have powerful cognitive systems evolved to acquire quite complex cultural knowledge from their elders. Adults and knowledgeable children also appear to be adapted to behave in ways that scaffold the learning of naive children (Csibra \& Gergely 2011; Dean et al. 2012; Kline 2015). The transmission of complex cultural information and its cumulative evolution requires accurate imitation (Tennie et al. 2009). Nonhuman social learners tested so far lack the skills necessary to acquire a large repertoire of complex skills, and there are so far no reports of nonhumans having acquired complex traits (traits composed of more than two or three separate innovations) by social learning. Human children routinely learn concepts with help from knowledgeable others that qualitatively transcend innate intuitions (Carey 2009). In the 1970s and 1980s, a highly innatist hypothesis for language learning was a major part of the cognitive revolution (Pinker 1994). It has failed to account, however, for the wide diversity of syntactic devices comparative linguists have since discovered (e.g., Evans \& Levinson 2009; Newmeyer, 2004). Most grammatical constructions appear to be cultural features underpinned only indirectly by rather general cognitive features (e.g., Hurford 2011). Research over the last two decades has produced a fairly detailed picture of how human imitation develops over the first 12 months of life (Carey 2009; Tomasello et al. 2005) and how older individuals support the imitative efforts of children (Csibra \& Gergely 2011). The same basic system seems to operate across all domains of culture (Harris 2012).

Human social learning contrasts sharply with that of chimpanzees and other nonhuman animals. Chimpanzee social learning focuses primarily on goal-related behaviors, such as retrieving food. In comparative studies with human children, chimpanzees tended to imitate only the behaviors necessary to reach a goal, while children diligently imitated all of an adult's behaviors whether or not they directly resulted in reaching a tangible goal (Whiten et al. 2009). If adults suggest that it is important, children will replicate adult actions in ritualistic detail (Herrmann et al. 2013). This finding initially seems counterintuitive-humans appear to unnecessarily "overimitate," while chimpanzees seem better able to boil down a stream of actions to only the necessary, goal-related behaviors. However, over-imitation is probably an essential adaptation for building a repertoire of elementary skills and maneuvers that will eventually be incorporated into complex crafts and social institutions (Harris 2012). Many cultural adaptations are so complex that the functional significance of many elements of them is opaque to learners and often to accomplished practitioners as well. Carefully following demonstrated recipes preserves functionality, albeit at some cost of replicating non-functional elements.

Thus, studies of child development strongly suggest that human social learning does accurately transmit cultural variants over a wide range of design space in many domains (Carey 2009). In section 4.2, we address the issue of the cultural transmission of social norms.
Many experimental studies of human social learning have been conducted on adults, mostly student volunteers (Kempe \& Mesoudi 2014). Individuals use a variety of strategies to acquire social information, tending to favor success-based strategies when such information is available to them; conformist strategies are typically employed when payoff-based information is unavailable or uninformative (Molleman et al. 2014).

\subsection{Descent with modification}

Darwinian evolutionary processes shape adaptations incrementally. Marginal change occurs over each small time step in history, followed by reasonably faithful transmission and further marginal changes. Over many such time steps, the evolving system can, but need not necessarily, change dramatically. Often successful lineages split, often repeatedly, to form many sister groups. The Romance language societies are an example. A necessary but not sufficient test of the CGS hypothesis is whether the social systems of human societies follow a phylogenetic pattern, the way genes do, thus demonstrating descent with modification. If the transformational processes operating in human history were as strong as the improvisational intelligence hypothesis (Cosmides \& Tooby 2001; Pinker 2010) holds, then humans would routinely switch such things as their political organization quite rapidly and in large jumps, thus not leaving a phylogenetic pattern. Similarly, the evoked culture hypothesis holds that human behavioral variation is based on a pan-human evolved psychology that is expressed in different ways in different environments (Tooby \& Cosmides 1992). If this hypothesis were true, phylogenetic patterns would also be weak or undetectable; variation would reflect current ecology, not cultural history. A pattern of descent with modification by itself does not test the CGS process against competing evolutionary processes acting at lower levels of organization than groups; it only establishes the action of an evolutionary rather than a non-evolutionary process.

Historical linguists established that languages evolved by descent with modification before Darwin and his contemporaries began to apply the idea to biological evolution (Müller 1862). Recently, phylogenetic methods, originally borrowed from biology, have been applied to detecting descent-with-modification patterns in cultural data (Mace \& Jordan 2011). These methods have reached a high degree of sophistication (e.g., Bouckaert et al. 2012). Language phylogenies appear to be a reasonable proxy for population movements, often matching phylogenetic patterns based on genes reasonably well (Cavalli-Sforza et al. 1988), albeit not perfectly.

The phylogenies of several large language families have been investigated, including Bantu (Holden 2002), Austronesian (Gray et al. 2009), Tupi (Walker et al. 2012), and Indo-European (Bouckaert et al. 2012). The Walker et al. study is notable for quantitatively estimating rates evolution in several Tupi cultural domains (usually less than five transitions in 10 millennia). From the correspondence of linguistic time-depth estimates and archaeological dates, each of these language families appears to constitute a cultural adaptive radiation based upon agricultural subsistence and associated social-organizational features. These innovations allowed their speakers to expand at the expense of hunter-gatherers in the Early and Middle Holocene. In the case of the eastern Austronesians, expansion was 
Richerson et al.: Cultural group selection plays an essential role in explaining human cooperation

partly to remote, previously uninhabited oceanic islands. Early farmers in Europe were clearly the result of a demic expansion (Haak et al. 2010). Currie and Mace (2009) found that the area over which a language is spoken is more closely related to its political complexity than to any other variable tested, consistent with the hypothesis that CGS on social organization features correlated with language played an important role in the creation of patterns of linguistic diversity. Historically attested expansions of empires, typically accomplished by a mixture of military conquest, assimilation of conquered peoples, and migration into the more prosperous dominant society, appear to exhibit all three CGS processes (Turchin 2006).

Language trees can be used as a foundation for studying other kinds of cultural evolution. Such studies show that features of social organization often include strong phylogenetic signals that are correlated with the linguistic signal. Again, this is a necessary but not sufficient condition for CGS to have been an important process. Guglielmino et al. (1995) studied 47 cultural traits, many of them related to social organization, in 277 African societies. They used correlation with language groups to estimate "vertical" (within-culture) transmission, similarity to neighboring groups to estimate "horizontal" diffusion from linguistically unrelated groups, and correlation with vegetation type to infer the effects of adaptation to local environments. They detected a strong effect of vertical inheritance of cultural traits related to family and kinship and the economy, and a medium vertical effect on traits related to social stratification and house type. The effect of horizontal diffusion was high on the division of labor by sex and house type. The direct effect of environments was strongest on economy-related traits and medium to low on other traits. In other words, the effect of either vertical or horizontal transmission was strong on all traits examined. Currie et al. (2010) used the rise and fall of political complexity in Austronesian societies to directly test the descent-with-modification hypothesis. They found that in the best-fitting model, political complexity increased and decreased in incremental steps. The second-best-fitting model allowed for decreases (but not increases) in complexity to skip intermediate steps.

As more historical and archaeological data are converted to time series suitable for quantitative analysis, tests of descent with modification will become more common and more precise (Turchin 2008). It should also be possible to estimate the parameters of mechanistic evolutionary models to such data to more directly test the CGS hypothesis and its competitors. See Bouckaert et al. (2012) for a simple example. Of particular interest will be investigations that trace the evolution of Roman law and similar institutions, as they were transmitted both vertically and horizontally. Certainly, no evidence carefully examined to date suggests that non-evolutionary patterns dominate the archaeological and historical record, as would be suggested by strong versions of the evoked culture and improvisational intelligence hypotheses.

\section{Culture exhibits group-level variation}

IfCGS processes are to operate on cultural variation, then we must document appreciable variation at the level of groups. Group selection on genes is generally implausible because migration between groups prevents substantial genetic variation from arising in groups larger than those composed of close kin. In section 2.1 we reviewed the mechanisms by which cultural systems can preserve much more variation between groups of unrelated or distantly related individuals than can genetic inheritance. Here we review the evidence that between-group cultural variation is large enough to support group selection and that the facility with which children learn the norms of their own group shows how the cultural inheritance system maintains CGS relevant variation.

\subsection{Quantitative evidence of cultural differences between groups}

The more groups differ, the more effective CGS is likely to be. The Price equation, a general depiction of selection operating on multiple levels, can be re-arranged to express the required conditions for a group-beneficial trait to be favored by selection in the face of individual-level selection against the trait (Bell et al. 2009; Bowles 2006):

$$
\frac{\text { Group Benefit }}{\text { Individual Cost }}>\frac{1-F_{\mathrm{ST}}}{F_{\mathrm{ST}}}
$$

where $F_{\mathrm{ST}}$ is the fraction of the total variance that is between groups. If groups do not differ at all for some trait, then $F_{\mathrm{ST}}=0$, and if the groups have nothing in common, then $F_{\mathrm{ST}}=1$. Developed to measure inbreeding and genetic differences between populations, $F_{\mathrm{ST}}$ can also be used to measure cultural differences. Equation (1) says that traits will be favored if they have zero individual costs and positive group benefits. Traits with some detrimental individual-level effect on reproduction and/or survival can also be favored if group benefits are sufficiently large to satisfy the inequality. Figure 1 describes the level of between-group variation required and plots the relationship between the minimum required benefit-to-cost ratio and the level of cultural differences represented in Equation (1). Note that the scope for group selection is sensitive to changes in low values of $F_{\mathrm{ST}}$, because the minimum condition declines geometrically with a linear increase in $F_{\mathrm{ST}}$. This relationship suggests that evolutionary forces that create relatively small differences between similar groups

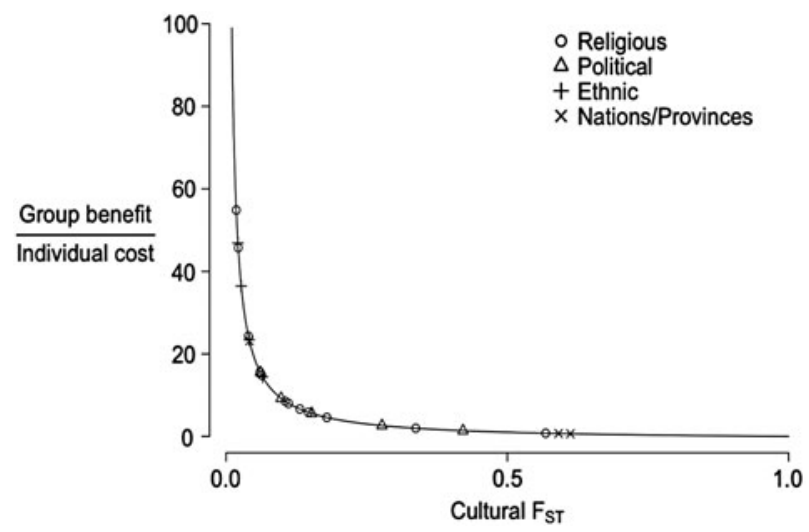

Figure 1. The scope for group selection. The curve is the minimum variation required for a group-beneficial trait to be favored by natural selection as a function of the level of cultural differences between groups $\left(F_{\mathrm{ST}}\right)$. The points are cultural $F_{\mathrm{ST}}$ measurements across several kinds of group identities. For details, see the appendix. 
Richerson et al.: Cultural group selection plays an essential role in explaining human cooperation

can provide some scope for group selection in cases where group benefits are high relative to costs.

Aside from a few cases (e.g., Edgerton 1971), computing cultural $F_{\mathrm{ST}}$ from ethnographic data is not possible because most ethnographers studying simple societies report only normative behavior and neglect individual variation. Ethnographers and historians tacitly assume that individual variation for normative behaviors is typically small within groups, is large between groups, and grows larger with time in most lineages. If this is correct, the scope for CGS is large. To test the assumption, we used publically available datasets to measure variation in normative beliefs and behaviors within and between contemporary populations. These data capture within- and betweengroup differences at various scales, from the national level to small political groups within larger communities.

We calculated the cultural $F_{\text {ST }}$ of a wide range of neighboring groups for a wide range of beliefs and behaviors (Fig. 1) using data from responses to international and state-level surveys, such as the World Values Survey, the AfroBarometer, the Public Policy Institute of California statewide surveys, the Scottish Health Survey, and the Anwar Sadat Chair Arab Public Opinion Surveys, among many others. Together, these datasets provide a comprehensive report of opinions and behaviors in group-level organizations that are important in human societies: political, religious, ethnic, geographic, and socioeconomic identities. Some measurements are based on responses to one or two questions in a survey, analogous to one or two loci for a genetic $F_{\mathrm{ST}}$. Others are based on many questions and thus reflect broader level differences between groups. All measurements select questions that relate to a particular theme relevant to group performance, such as attitudes toward immigration, a state-controlled economy, or the adoption of strict religious codes. Details can be found in the table in the appendix of this article.

Consistent with the hypothesis that individual variation for many normative beliefs and behaviors is small within groups and large between groups, we find large betweengroup differences among all groups of all scales (Fig. 1). Among small political or ethnic groups, nations, and religious majorities, we consistently report $F_{\mathrm{ST}}$ values greater than 0.01 , with some traits showing $F_{\mathrm{ST}}$ scores above 0.6. These values are important, as small increases in cultural $F_{\mathrm{ST}}$ beyond 0.01 increase the possibility for CGS dramatically. Our data reach well into the plateau of the L-shaped region of the group selection condition.

The large cultural differences measured in this analysis suggest a wide scope for CGS. Our average measurements of cultural $F_{\text {ST }}$ place the group-benefit-to-cost requirement well within range of some important cultural behaviors. As described in section 5 further on in this article, some cultural variants provide a distinct advantage over other groups, outweighing the individual costs of implementation. Bell et al. (2009) compared cultural $F_{\text {ST }}$ from the World Values Survey with genetic $F_{\text {ST }}$ from Cavalli-Sforza et al.'s (1994) classic study. For neighboring groups that might actually compete, cultural $F_{\mathrm{ST}}$ is approximately an order of magnitude larger that genetic $F_{\mathrm{ST}}$. These data suggest that the scope for CGS is substantially higher than for genetic group selection.

\subsection{The transmission of social norms}

Social norms are among the cultural traits that children readily acquire from adults and other children. Young children are strikingly inflexible with social norms and rules, resulting in young moral absolutists (Levy et al. 1995). Human children are also motivated to engage with others to pursue a shared goal without any reward, apart from the enjoyment of engaging with another person socially (Tomasello \& Carpenter 2007). For example, children as young as 14 months will readily provide instrumental help to an adult, and children of 18 months will help and cooperate with unfamiliar adults. Chimpanzees' abilities in this regard are more limited (Warneken \& Tomasello 2009). Attempts to raise chimpanzee infants as if they were children are unsuccessful because young chimpanzees rather quickly mature into independent individuals that are not very responsive to being taught social norms, much less adopting them spontaneously (e.g., Hayes 1951).

Not all potential providers of social learning are equal. Even very young children prefer teachers and social partners who belong to their own social group. For example, preschool children preferred to learn both linguistic and non-linguistic information from individuals who spoke with the same accent as themselves (Kinzler et al. 2011), and 12-month-olds preferentially copy the food selection choices of adult native speakers of their own language (Shutts et al. 2009). The effects of out-group discrimination and in-group preference based upon cultural categories like race are evident as early as 3 years of age, when children begin to implicitly associate same-race faces with positive facial expressions and different-race faces with negative facial expressions (Hugenberg \& Bodenhausen 2004). Children show adult levels of racial in-group bias by age 6 (Dunham et al. 2008), although language difference seems to be a more intuitively salient marker than differences in skin color (Kinzler et al. 2009).

By the time people are adults, much experimental work by social identity theorists shows that, in many social situations, individuals' behavior is substantially influenced by their affect-laden social group identifications (Haslam 2001). "Who are we?" is an important component of the question "who am I?" The accurate imitation of cultural variation, especially group identities and social norms, is the basis for heritable cultural variation between groups upon which CGS can act. Social learning biases, such as those described previously, act as a general force adapting culture to local environments. They tend to preserve locally successful social behaviors and reduce the diffusion of cultural variation from one social group to another (see sects. 2.1 and 6.1). Identification with a group sometimes results in derogation of other groups. Attitudes toward out-groups are certainly not invariably invidious, however, contrary to some theories of ethnocentrism (Brewer 2007).

Norm transmission has not been extensively studied in adults or undergraduate volunteers. However, early experiments showed a strong conformity effect (Jacobs \& Campbell 1961), and rather firm socialization pressure with normative content is observed in some experiments (Baum et al. 2004; 2012). Insko et al. (1983) demonstrated the evolution of institutions in a multi-generation experiment designed to mimic the origin of states. Efferson et al. (2008) showed experimentally that symbolic markers and in-group favoritism coevolve. Derex et al. (2014) showed that cultural transmission increases 
Richerson et al.: Cultural group selection plays an essential role in explaining human cooperation

substantially in between-group competition compared to within-group competition treatments.

\subsection{Institutions are group-level cultural traits}

Scott (2001, p. 48) provides a formal definition of institutions: "Institutions are social structures that have attained a high degree of resilience. [They] are composed of cultural-cognitive, normative, and regulative elements that, together with associated activities and resources, provide stability and meaning to social life." Institutions are important because they create and maintain variation between groups while simultaneously damping down variation among individuals within groups. Institutions only function when large numbers of people conform to them. Institutional economists often characterize institutions as equilibrium solutions to social games that include rewards and punishments that act to induce individual conformity to the institution's norms (Aoki 2001; Greif 2006). As a system of commonly accepted norms, they are a property of a group and often vary qualitatively from group to group. Institutions are probably an ancient feature of human social life, ancient enough to have generated the coevolution of genes related to social behavior. The social life of living hunter-gatherers is certainly heavily institutionalized (Hill et al. 2009).

Game theory tells us that in repeated games without fixed end points, the number of equilibria multiplies inordinately. This is easiest to see in the case of institutions involving moralistic punishment. If punishing is sufficiently cheap for punishers and sufficiently costly to the punished, punishment can stabilize any behavior (Boyd \& Richerson 1992b). The empirical literature on village-scale commons management is particularly well developed and shows that the institutional diversity predicted by theory is amply manifest on the ground (Ostrom 2005). Similarly, ethnographers have documented ample institutional diversity between small-scale societies that are our closest living approximations to Late Pleistocene societies (e.g., Jorgensen 1980). Modern institutions vary substantially cross-culturally (e.g., Nivette 2011).

Henrich et al. (2004; 2006) have systematically investigated cross-cultural variation using experimental games, including dictator, ultimatum, third-party punishment, and public goods games. These studies suggest that institutional variation creates substantial differences in behavior. For example, Paciotti et al. (2005) conducted ultimatum game experiments in a Tanzanian village with two ethnic groups. The Sukuma, who are an expanding population of agro-pastoral colonizers, were found to have one of the highest average offers in the ultimatum game ever measured. The Pimbwe, in contrast, made quite low offers. Interestingly, the Sukuma have a unique, elaborate institution of village governance, which the Pimbwe lack, and the Sukuma organize the provision of public goods much more effectively than the Pimbwe.

Herrmann et al. (2008) compared the behavior of participants in public goods game experiments in 16 diverse societies. Participants were given the opportunity to punish other members of their group. Groups from societies that achieved high rates of cooperation over the 10 rounds of the game had high rates of punishment of non-contributors. However, in some societies, participants sanctioned high contributors as well as low contributors. In such societies, rates of cooperation in the games were low. The authors also compared the 16 societies by using responses to the World Values Survey to construct indices of civic cooperation and rule of law. They found that societies where experiment participants used punishment to promote cooperation also scored higher on these indices than societies in which some experimental participants punished cooperators.

In section 2.1, we outlined how various mechanisms can generate and maintain between-group variation in the face of migration. One important institutional mechanism with substantial empirical support is the marking of group members through differences in dialect, dress, religion, and similar symbols. These markings are often readily observed and hard-to-fake symbols (e.g., Iannaccone 1994) that are emotionally salient to their bearers. Symbolic markers thus serve to identify members of distinct social groups and subgroups and to define who is and who is not entitled to benefits from the activities of the organization.

Institutions from one group are often quite difficult for other groups to copy. The tendency of cultural items to diffuse between groups is favored by "observability" and "trialability" (Rogers \& Shoemaker 1971). The institutions of another society are often hard to observe because outsiders can seldom see the entire system of rules working together. Institutions are hard to try out, because for an institution to be effective, some critical mass of group members has to adopt its rules. Considerable collective decision-making and learning effort must be expended to integrate a novel institution with the other institutions of the adopting society. Greif's (2006) study of the evolution of early modern Mediterranean trading systems notes that institutional evolution occurs when ongoing changes destabilize an equilibrium previously regulated by an institution. Evolution then takes the form of a collective, culturally local search for a replacement institution. For example, the Genoese trading system was long regulated by personal reputations. As the Genoese traders grew wealthy, reputation monitoring became too time-consuming to mesh with the increasing management responsibilities of traders. Eventually they settled on a familiar formal legal system modeled on Roman law as preserved by the Church in the form of canon law.

If culturally transmitted institutions had a major coevolutionary impact on the innate components of our social psychology (see sect. 2.2), then for some part of our evolutionary history humans must have lived in institutionally structured groups of a few hundred to several thousand individuals that were symbolically marked and shared a common identity. Some authors argue that institutions only arose in the Holocene (Runciman 2005), although archaeological evidence and ethnographic insights from living foragers suggests that tribal-scale societies were common in the Pleistocene. Even the Shoshone, often used as an example of the simplest sort of family-band society (Steward 1955), had supra-familial institutions involving upwards of 15 separate families to cooperatively hunt in winter camps and a system for incorporating stranger families into these camps (Murphy \& Murphy 1986); because of the high mobility of families, winter camps regularly incorporated co-ethnic strangers. The Ju/hoansi (!Kung), another primarily familyband society, frequently organize at the camp- and regional-level when drought forces them to aggregate at permanent water holes (Lee 1972). In 32 present-day foraging societies, Hill et al. (2011) found evidence of frequent inter-band relations, low group genetic relatedness of co-residents within a band, and substantial interaction networks consisting of 
Richerson et al.: Cultural group selection plays an essential role in explaining human cooperation

unrelated individuals, all structured by institutions that differ from society to society. Even "acephalous" societies (those lacking formal leadership roles) use egalitarian institutions to create societies with quite large-scale activities (Mathew \& Boyd 2011). See section 5.1.

How similar modern foragers are to Pleistocene foragers is difficult to say with certainty. However, a reasonable assumption is that Late Pleistocene foragers exhibited a range of institutional complexity comparable to modernday foragers. Archaeological data suggest that during the Middle Paleolithic to Upper Paleolithic transition, about 45 k.y.a, coherent cultural groups with stylistically different technology were moving about the Western Eurasian landscape (Tostevin 2013). African evidence suggests that human use of symbolic markers goes back to about 164 k.y.a (Marean 2010). CGS could well have been an active process as far back as symbolic marking is evident.

The strategies used to formulate tribal institutions are widely applied to modern organizations such as churches, villages, neighborhoods, clubs, business organizations, and bureaucracies. For example, class and regional variations are usually marked by linguistic differences (Labov 2001). These institutional differences among groups in turn facilitate CGS via any of the three processes identified in section 2.1. The increase in institutional size and complexity in many societies in the Holocene is striking, but the evidence does suggest that the organization of anonymous societies using institutions has roots in the Pleistocene.

A theoretically significant set of institutions are those that regulate family life and small-scale partnerships. Mathew et al. (2013) note that humans routinely engage in a broader set of small-scale cooperative activities than do our primate relatives, and they argue that institutionalized third-party rewards and punishments often regulate such activities. Marriage and kinship are examples. Almost all cultures have one or a small number of standard, legitimate marriage contracts. Mating according to such a contract confers social benefits of the cooperative breeding system on the couple, and mating outside it is often significantly sanctioned. Kinship institutions specify the responsibilities of family members toward each other. Dispute resolution is an example of the institutionalization of small-scale reciprocity. Many societies have such institutions of varying degrees of formality (Nader 1969). If human cooperation could rest entirely on innate adaptations for kin cooperation and reciprocity, then why should these intimate relationships require institutional support? More likely, institutional support is required if people are to enjoy the benefits of highly cooperative interactions even in small social units. Marriage is a credible signal of commitment, for example, because of institutionalized third-party enforcement. Large groups will benefit from maintaining institutions that encourage their members to cooperate at the level of small groups or families to the extent cooperation at such scales is important economically and to the quality of child rearing. For the case of marriage, see Henrich et al. (2012b); for dispute resolution, see Gambetta (1993).

\section{Social groups in competition}

Here we consider examples of group competition in modern humans through the three processes outlined in section 2.1: selective borrowing between groups, migration of individuals between groups, and selective extinction and proliferation of groups. This evidence suggests that CGS occurs wherever significant cultural variation exists between neighboring and competing groups.

\subsection{Warfare}

The two conditions needed for warfare to cause CGS are (1) that variation in cultural traits between groups influences variation in success in warfare, and (2) that variation in success in warfare influences the spread of cultural traits. Losers of military competitions are sometimes killed outright and are sometimes voluntarily or involuntarily incorporated into the victorious society. Success and failure are often due to variation in the competing societies' technology, norms, and institutions. Losers or potential losers will also often attempt to emulate the technology and institutions of stronger societies.

Humans can make war because we solve collective action problems at large scales. Combatants experience the hazards of war, but the benefits of victory are widely shared, even by those who avoid the risks. Human societies that successfully suppress and sanction within-group violence, can still suffer high mortality from intergroup conflict. For instance, in the Turkana, a tribe of about one million people living in northern Kenya, $1 \%$ of mortality among Turkana men is from violence among Turkana, whereas $50 \%$ is from inter-ethnic raids (Mathew \& Boyd 2011).

Formal models indicate that genetic group selection can only support warfare in groups of, at most, a few dozen individuals (Choi \& Bowles 2007). However, lethal intergroup violence occurs between large human groups within which genetic relatedness is low and individuals do not know each other personally. For example, one WWII engagement alone, the Battle of Stalingrad, involved more than 1.7 million soldiers and produced over two million military and civilian casualties (Glantz \& House 1998). CGS can favor the evolution of warfare between cultural groups comprising hundreds to millions of mostly genetically unrelated individuals.

Even in politically un-centralized societies, CGS on simple institutions can support large-scale conflicts. The Turkana frequently raid neighboring ethnic groups for cattle (Mathew \& Boyd 2011). The scale of such raids can be very large despite the absence of hierarchical institutions. The average raid includes 315 men, with some raids including up to a thousand. Most participants are not close kin, and participants come from a wide swathe of Turkana society, including multiple sub-territories. This means that reciprocity based on warriors' reputations cannot by itself maintain cooperation in the raiding party. Instead, the Turkana have cultural norms that discourage cowardice, norms that are enforced by peers who sanction violators in their local group. In vignette studies, Turkana raiders had very positive attitudes toward warriors who raided animals from another ethnic group and very negative attitudes toward those who raided another Turkana territory, even though the respondents' own territory would benefit from the spoils in both situations. This pattern strongly supports CGS operating among the Turkana and neighboring groups having similar patterns of ethnolinguistic-scale cattle raiding. The common ethnic identity of Turkana combined with effective norms 
Richerson et al.: Cultural group selection plays an essential role in explaining human cooperation

and institutions are the proximate cultural mechanisms that make large-scale raiding possible.

Archaeological and ethnographic data suggest that warfare occurred in a sufficiently wide range of societies for long enough (Gat 2008) to be a gene-culture coevolutionary force. Warfare occurs in societies pre-dating agriculture and sedentary living (Keeley 1997). Nevertheless, the direct evidence for warfare in the Pleistocene is very limited, and doubts about war's importance before the Holocene persist (Guthrie 2005).

As a consequence of warfare, the cultural traits of the successful groups often spread and the cultural traits of defeated groups and polities often decline. Similarly, groups with institutions that more effectively foster cooperation are most likely to be the victor (Turchin 2003; 2006). Victorious groups often expand their borders and absorb conquered peoples. The Mongols (Turchin 2006) and Nuer (Kelly 1985) are examples of militarily successful societies that absorbed defeated groups. Absorption may be passive, in which defeated people adopt the identity of the dominant group through marriage and/or migration, or it can be coercive. Slaves captured by the Comanche, and their subsequent offspring, became Comanche themselves after one or two generations (Hämäläinen 2008). Cultural systems associated with Islam and Christianity have also spread in part through military conquests, facilitated by coercive as well as voluntary conversion of peoples dominated by the victors. Soltis et al. (1995) estimated group extinction rates from the differential group extinction and conversion of losers in a sample of societies in Papua New Guinea. They found that the estimated extinction rates would allow a group beneficial cultural trait to spread to most of a meta-population in 500-1,000 years. This is consistent with the gradual increase in the complexity and size of societies over the last 10 millennia.

Variation in economic systems and political institutions between societies also affects military success. Agriculturalists find it easier to stockpile resources to feed standing armies than herders (Turchin 2006). Tilly (1992) argues that sovereign territorial states out-competed both empires and feudal societies because they were better able to raise capital for military campaigns.

Over the past 200 years, democracies have been replacing autocracies. Part of the reason may be because democracies have been more likely to win wars (Reiter \& Stam 2002). Democratic leaders seem to be better at picking the wars they fight, and they are more politically accountable when wars are lost. Soldiers from democratic nations also seem to fight more effectively than soldiers from non-democratic nations, even after controlling for wealth and other measures of power, perhaps because they more consistently report that they find their government's war policies legitimate (Reiter \& Stam 2002).

To summarize, warfare clearly demonstrates the requirements for CGS. Cultural traits that affect military success are highly variable, and success in war tends to spread such traits by all three forms of CGS.

\subsection{Competition between economic organizations}

Competition between human organizations is certainly not restricted to violent conflict. Economic competition is an important and typically peaceful form of CGS. Economic CGS has a long history (Curtin 1984) and is very important in modern societies. It is partly responsible for ongoing rapid evolution in the domains of organizational rules, structure, business practices, and technology.

Economists have noted that competition among firms creates a Darwinian dynamic (Nelson \& Winter 1982; Schumpeter et al. 1934; Veblen 1898). Well-run firms profit and survive, while poorly run firms with losses expire. This selection of firms in the market was part of what Schumpeter et al. (1934) called "creative destruction." Some of the success and failure of individual firms is caused by the structure, management style, institutions, and other aspects of the culture of the firm itself. Business organizations have been a focus of social identity research and research on the impact of social identity on organization performance (Ashforth et al. 2008). New employees acquire the firm's culture through formal and informal socialization and incorporate the firm's culture as part of their social identity. When those cultural differences influence a firm's success, CGS will occur. Nelson and Winter's (1982) highly cited classic argues that much of the important know-how influencing firm success is tacit, embedded in the skills of individuals and informal routines of interaction. This information is hard to acquire by borrowing from successful firms and even selective hiring. Hence, cultural differences between firms tend to persist, and differential expansion and contraction of firms is very active despite attempts to acquire the practices of more successful firms.

Economists routinely study all three mechanisms of CGS in competitive market systems. The differential proliferation (market entry and split) and extinction (market exit and bankruptcy) of firms influences the future population of firms and frequencies of business practices. Empirical studies of selection on economic organizations owe much to the pioneering work of Hannan and Freeman (1993), which has attracted thousands of citations. An example is the evolution of the synthetic dye industry in the 19th century (Murmann 2003). The earliest synthetic dye firms were British, but German firms lobbied provincial governments to support research chemists in their universities. The students of the chemists often found jobs in German dye companies, leading to the development of the first industrial research and development (R\&D) laboratories. As a result, German companies were able to out-innovate their British counterparts, and by 1900 they dominated the global market. Inspired by successful large-scale $R \& D$ during WWII, British and American governments, universities, and firms started to copy German R\&D institutions on a large scale (Murmann 2003).

The natural selection of firms occurs when corporate cultures do not match the needs of the external environment (e.g., McGovern 2007). Valuable information also flows between firms through selective imitation and best-practice adoption (e.g., Bolton 1993; Haunschild 1993) as well as patent licensing and corporate espionage. Information also migrates with employees through processes such as talent raiding (Gardner 2003) and spin-off companies. Klepper and Sleeper (2005) showed that, in the laser industry, spin-off companies do indeed inherit the capacities of their parent organizations.

Substantial quantitative evidence for CGS within economic systems comes from research on competition between technology firms. For example, Derfus et al. (2008) observed that the market for hard disk drives created a headlong competitive race to stay near the 
Richerson et al:: Cultural group selection plays an essential role in explaining human cooperation

forefront of technology; failure to keep up led to bankruptcy. Such competition led to various market-level outcomes, such as leapfrogging, in which organizations competing from a lagging position were more responsive to competition than better-positioned competitors (Barnett \& Pontikes 2008). A history of competitive success tends to create a tendency for increased mistakes. Higher failure and exit rates have been observed in companies with less competitive experience, such as monopolists (Barnett \& McKendrick 2004). Murmann (2003) found that the originally dominant British dye firms suffered through their lack of exposure to competition. A higher rate of market entry and exit in the German dye industry led to a greater exploration of different business practices, resulting in greater variation and stronger selection. The success of Silicon Valley derives from Valley-wide "ecosystemic" institutions that accelerate all three forms of CGS (Hwang \& Horowitt 2012). For example, new firms form easily in order to exploit promising ideas, and investors, entrepreneurs, and founding employees expect that most will fail. An efficient system of gossip ensures a general awareness of individuals' reputation, reducing the risk that the high mobility generated by rapid firm turnover will be exploited by unscrupulous individuals.

Modern nations intentionally regulate and promote CGS in the economic domain (Motta 2004). Regulators force businesses to compete through anti-monopoly legislation. Patent systems attempt to induce the socially optimal amount of R\&D. During the patent period, firms can recover large $R \& D$ costs, but eventually everyone can use the off-patent innovation. Collusion and anti-poaching agreements between companies are outlawed in order to guarantee competition. International free-trade agreements attempt to limit national protection of domestic companies at the expense of foreign competitors. Therefore, the mechanisms of CGS are not just present in modern economies, but are the deliberate focus of institutional design at the national and international level. In general terms, these policies, at least in principle, benefit ordinary citizens by tailoring economic group selection to meet common social goals. These national regulations may themselves constitute another level of organizational selection above the firm, in which governments, industries, and other domestic organizations are selected over time via differential success, learning, and migration between countries. The interplay between CGS at the level of the firm and the level of the nation is the subject of much national and international political discussion.

\subsection{Religious competition}

The action of CGS on variation in religious institutions has been observed in a number of historical and contemporary populations, often resulting in the expansion of one cultural group relative to others. Religious ethical systems are often integrated into the basic norms and institutions of a society. It is common for different religions to have different birth or death rates, and they often compete for converts.

Scholars have suggested many examples of the role of religion in competition between groups. The advent of "Axial Age" universalistic religions around 500 B.C.E. created overarching ethical systems. They introduced the innovation of doctrinal mode rituals that could unite formerly disparate cultures and thus favored expansion of empires to units large enough to resist Central Asian invaders, who had perfected horse archery (Turchin 2009; Whitehouse 2002). Christians in the Roman Empire were more likely to practice mutual aid and economic cooperation than non-Christians (Stark 1997). Christian cooperators positively assorted through association in spiritual communities. Non-cooperators were punished with ostracism. This combination led to greater success in dealing with resource scarcity and disease and thus to higher survival, reproduction, and conversion rates that eventually led to Christianity being the state religion of the Empire and its successor polities. Early Islam promoted strict sacred norms of fairness and unity among co-religionists, leading to its remarkably rapid expansion. Reduced small-scale tribal warfare, increased external defense, and the creation of institutions that greatly facilitated long distance trade facilitated material success, intergroup military success, and large conversion rates (Ibrahim 1990).

Recent history suggests that competition between groups based on variation of religious belief and ritual practice continues today. An analysis of demographic data from more than 800 communes in the United States over a 200-year period revealed a strong correlation between costly ritual displays and the longevity of the commune (Sosis \& Bressler 2003). Finke and Stark's (1992) study of U.S. religious history documented an overall increase in churchgoing since independence, as well as large shifts among denominations. Hout et al. (2001) conducted a pioneering quantitative micro-evolutionary study of the relative expansion and contraction of Protestant churches in the United States during the 20th century. They found that variation in birth-rates rather than conversion rates mainly drove the changes. Pronatal norms and strong cultural isolation are leading to rapid demographic expansion of the more traditional or "Old Order" Anabaptists (Kraybill \& Bowman 2001) relative to almost all other North American subcultures. The Anabaptist Hutterites are North America's most successful communal movement. They have very high population growth rates resulting from strongly pronatal norms and low apostasy, arguably caused by their strongly isolationist practices (Sato et al. 1994). The number of Evangelical Protestant churches increased relative to that of Roman Catholic churches in Latin America during the 20th century, mainly due to their stronger emphasis on missionary work (Stoll 1990).

Not surprisingly, given the breadth of evidence, religious variation is frequently cited as one of the most important examples of CGS (e.g., Wilson 2002).

\section{Evidence for group-level functionality}

If CGS has operated on human cultures directly, and indirectly on human genes via gene-culture coevolution, then we would expect to find clear examples of design for group function. Arguments for group selection in humans have often stressed the evidence from design (e.g., Wilson 2002), whereas we have so far stressed process-level evidence. However, the evidence from design is also important and compelling. We discuss language, social control, and religion as examples.

\subsection{Language}

Language reflects the operation of CGS in three different ways. First, language is necessary for sophisticated norms 
Richerson et al.: Cultural group selection plays an essential role in explaining human cooperation

and institutions to function. Second, language only functions if there is a considerable degree of trust among speakers, who comprise groups far larger than families. Third, language differences are among the most important markers of group boundaries, and limited linguistic communication makes the diffusion of institutions between societies more difficult.

Humans use language to formulate and transmit social norms and to operate institutional systems of reward and punishment that stabilize these norms. Maynard Smith and Szathmáry (1995) nominated language as the key innovation leading to human societies arriving at a new level of biological organization. Smith (2010) reviews the ample evidence that language plays an important role in solving collective action problems. Norms and institutions are articulated publicly so that individuals can understand them. Individuals who appear not to understand them are typically subjected to verbal reprimands. Institutional change is advocated and discussed in speeches and codified by verbal consensus. In complex societies, written records evolved to make possible quite intricate institutions. Even the most selfish political operators usually take care to frame their public arguments to give the impression that they are furthering the general welfare.

Much variation in norms and institutions is deeply embedded in the vocabulary and syntax of languages (Wierzbicka 1992). Such linguistically encoded information is often tacit knowledge that is shared by speakers but diffuses with difficulty to other languages. Language differences thus help preserve the between-group variation that CGS acts on. Expanding language families often mark the expansion of a complex of technical and social innovations; the Indo-European, Bantu, Tupi, and Austronesian families are examples, as we have noted in section 3.2.

The evolution of the capacity for languages that can serve cooperative functions is a considerable puzzle, because the existence of human language presupposes cooperation (Hurford 2007). Communication systems in animals are largely restricted to costly signals or situations where deceptive signals can be detected and punished (Lachmann et al. 2001). In such systems, very little information is transmitted and syntactic complexity is quite limited (Hurford 2007). Unless listeners are able to trust speakers more often than not, a complex symbolic language would be useless. If most speech acts were self-serving and/or deceptive, listeners would not bother to listen. Thus, at least a modicum of trust and cooperation seems necessary before a low-cost high-fidelity communication system could begin to evolve. Scholars often assume that language is the premier mechanism for cultural transmission. If so, how could culture itself, much less CGS, ever have gotten off the ground?

How could CGS have existed before language? A plausible answer is that culture was already tolerably complex before the capacity for language began to evolve. Stone tool manufacture may go back three million years, and edge-wear evidence suggests that early stone tools were used to make a toolkit of perishable materials such as wood and hides. Merlin Donald (1991) hypothesizes that Homo erectus and related Lower and Middle Pleistocene hominins were talented mimics, and that mimesis served as the evolutionary basis on which language could subsequently evolve. Donald reviews evidence that 19thcentury deaf-mutes could learn complex manual skills by imitation alone. They also acquired basic social norms and integrated themselves into complex institutions. Dances and similar motor rituals are among the things that pre-linguistic but highly mimetic humans could likely acquire. Such rituals today have powerful group-bonding effects and are therefore commonly incorporated into religious and secular patriotic celebrations (see sect. 6.3). Thus, a scenario in which the capacity for complex trustworthy language evolved by CGS and culture-led geneculture coevolution (see sect. 2.2), is plausible. All the recent evolutionary theories of the capacity for language, beginning with Pinker and Bloom's (1990) pioneering article, are culture-led gene-culture coevolutionary hypotheses. Theories differ mainly in the extent to which genes have responded to the coevolutionary pressure with language-specific cognitive structures.

Language diversity suggests that language is an important component of CGS. The stunning diversity of languages shows that differences between languages readily evolve (Nettle 1999). Why? Following the arguments for the efficiency of having innate, information-rich cognitive modules (Tooby \& Cosmides 1992), having tight constraints on languages ought to make them easier and quicker to learn both for children and for people wanting to be able to speak multiple languages. It is easy to imagine how tight, innate constraints might affect language (Sampson 2005), but no evidence of such constraints has been observed. Languages are highly variable in every element that has been studied closely (Newmeyer 2004). It might be that language differences, like other symbolic differences, evolve to limit communication between groups (see sect. 2.1). Sociolinguists find that small but socially significant dialect differences emerge rapidly along social fault lines, implicating the active evolution of symbolic marking rather than, or in addition to, drift-like differentiation of languages (Labov 2001). Leaders of dialect evolution are typically individuals with high local prestige. Social identity theorists have shown leader charisma to be essentially a recognition by followers that a leader is prototypical of the in-group (Steffens et al. 2014). The ensuing emotional identification of followers with their leaders probably leads to followers adopting the speech variants of such leaders. The evolution of symbolic boundaries to communication will help protect group-level cultural variation from the homogenizing effects of migration, and thus favor the operation of CGS.

Linguistic variation is extremely important in maintaining social structure and influencing social judgments, as we saw in the case of children's early learning biases (sect. 4.2). In small-scale societies, language differences generally mark the boundaries of groups that conflict (Nettle 1999). It is as if CGS favors not being able to understand those whom you likely cannot trust. Even at the scale of businesses and similar groups whose members speak the same language, group-specific jargon often distinguishes insiders from outsiders. Thus, an innate capacity for the rapid cultural evolution of linguistic and other symbolic differences plausibly coevolved with group selection on cultural variation (see sect. 2.2). Language variation helps maintain between-group variation in other cultural domains, contributing to CGS, but it is also likely to be a product of it.

\subsection{Crime, deviance, and punishment}

Violent or peaceful competition between organizations depends upon the preservation of between-organization 
Richerson et al.: Cultural group selection plays an essential role in explaining human cooperation

differences, and to compete successfully, an organization must solve dilemmas of cooperation among its members (Wilson 2002). CGS will tend to favor societies that produce public goods like defense, transportation infrastructure, and a fair legal system. One of the most important public goods produced in human societies are institutions of order and justice that protect individuals from predators and public goods from free-riders. All successful small-scale common property systems have institutions for monitoring and punishing defectors (Ostrom 1990). In contemporary societies, measures of culturally inherited trust are correlated with economic performance (Algan \& Cahuc 2010). Theoretical models (e.g., Boyd et al. 2003) and experiments (Fehr \& Gachter 2002) suggest that moralistic punishment can be a very effective mechanism for promoting cooperation. Thus, we expect that the quality of institutions regulating crime and deviance will play a significant role in the performance of human organizations. In experimental settings, people readily migrate from model societies that do not have sanctioning institutions to join ones that do (Gürerk et al. 2006). In the coevolutionary long run, social institutions of monitoring, reward, and punishment were probably the main factors exerting negative selection on genes that promote antisocial behavior and positive selection on genes that lead to prosocial dispositions (see sect. 2.2).

Punishers do not even need to be cooperative themselves to furnish some of the benefits of cooperation (Eldakar \& Wilson 2008), but many experiments have detected altruistic punishment (Fehr \& Gachter 2002). An important theme of our argument is that the question of altruism as a proximate mechanism is not central to the issue of CGS. CGS can favor reputational and other motivational systems that alter individual incentives, thereby reducing or eliminating the need for altruism (Fehr \& Henrich 2003; Panchanathan \& Boyd 2004). Altruistic behaviors have been the focus of models and experiments because they are the hardest ones to account for, not because they are empirically the most important. Careful experimental attempts to expose covert selfinterested motives have found support for an "empathy altruism” hypothesis (Batson 2011). In experimental treatments designed to induce research participants to empathize with someone who is going to suffer a harm, many people will volunteer to help the victim at a cost to themselves. Batson's program of experiments was designed to show that various egoistic hypotheses could not entirely explain these results. Models suggest that the evolution of altruistic punishment is likely to be frequency dependent (Boyd \& Richerson $1992 \mathrm{~b})$. If punishment is sufficiently costly to the punished and cheap to the punisher, a relatively few punishers suffice to encourage reluctant cooperators and would-be defectors to cooperate. Experimental studies using economic games support the findings of models. They find much individual variation both in propensities to cooperate and to punish non-cooperators (Henrich et al. 2010a; Kurzban \& Houser 2005). Only a minority of participants act altruistically. We are inclined to think that evolution has built an element of genuine empathy and true altruism into our social psychology because monitoring and punishment are costly to punishers and to punishees, but many people obey norms mainly because deviance is punished.

Historical and ethnographic data suggest that variation in the effectiveness of controlling self-seeking behavior is related to all three forms of group selection. For example, Turchin and Nefedov (2009) studied dynastic cycles in agrarian societies. Elite groups do provide services in such societies, but they have a tendency to grow and become increasingly corrupt over time. Corrupt elites tend to be replaced by competitors who provide better governance. Successful empires, such as those of ancient Rome and Han China, had more effective institutions for producing public goods and incorporating new populations into their expanding polities than their competitors. The increasing acceptance of the rule of law in Early Modern Europe is fundamental to that region's explosive demographic and economic growth (North et al. 2009). In the modern world, large numbers of migrants move from countries with low norms of civic cooperation and poor rule of law to countries with more civic cooperation and better rule of law (Boyd \& Richerson 2009). Thus, we have ample evidence that internal regulatory systems control deviance and solve dilemmas of cooperation to varying degrees of effectiveness. Much of the last five millennia of human history can be read as a slow, halting increase in the effectiveness of such institutions (e.g., Fukuyama 2011).

\subsection{Religion and ritual}

Religions furnish group-boundary-defining symbols, moral justifications for institutions of social control, and rituals that celebrate and facilitate an organization's solidarity. Religions contain effective mechanisms for reducing internal variation through sacred principles and norm-enforcing rules of reward, punishment, and exclusion, which bind participants into moral communities (Atran 2010; Graham \& Haidt 2010; Rappaport 1999). Numerous studies have found evidence for group-functional properties of religions (Atran 2002; Atran \& Norenzayan 2005; Boyer 2001).

The success of a number of key cultural innovations that generate well-documented associations between in-group prosociality and religious/ritual participation appear to be clear examples of group functionality (Henrich et al. 2010a; Norenzayan \& Shariff 2008). Belief in moralizing high gods is associated with cooperative altruism (Johnson 2005; Shariff et al. 2009) and willingness to punish non-cooperators (McKay et al. 2011). Ethnographic, historical, and experimental evidence suggest that synchronized dance and rhythmic ritual facilitate coordination and bonding of groups, and relieve low-level in-group conflict (Kirschner \& Tomasello 2010; McNeill 1995; Rappaport 1999). Theoretical arguments (Iannaccone 1992), supported by quantitative observations (Sosis \& Bressler 2003; Sosis \& Ruffle 2003), suggest that costly signals of group membership, such as hard-to-perform rituals and other sacrifices, help groups weed out free-riders and foster ingroup altruism. A common characteristic of religions is sacred rules (Durkheim 1912; Rappaport 1999). They supply a stable and enforceable moral code, held as inviolable by religious adherents (Atran 2010). These often manifest as prosocial rules, including norms of fairness and norms of group defense, and also as symbolic systems that demarcate in-group membership. At the same time, of course, religions (and other ideologies), when used to create in-group solidarity, sometimes create fear and hatred of out-groups (West 1941). CGS is not utopian!

Non-religious institutions also carry out rituals as part of their group culture, and they have similar effects. McNeill (1995) gives evidence that the ritual of close-order drill 
Richerson et al.: Cultural group selection plays an essential role in explaining human cooperation

reshaped warfare by facilitating in-group cooperation and camaraderie, leading to the higher success of groups that perform these seemingly functionally meaningless rituals.

\section{Conclusion}

The evidence we have outlined in this article makes the case that culture varies between human groups ranging in size from organizations within societies to ethnolinguistic tribes and nations, and even multi-ethnic empires. These groups are often symbolically marked, have more or less formal criteria for membership, and possess distinctive social institutions. This variation is transmitted to new generations by the human capacity to acquire norms and social institutions via imitation and teaching. Institutions include mechanisms of selective punishment and reward that reduce small-scale variation in behavior. Human organizations compete, often in highly organized ways, and the success and failure of groups in such competitions is often determined by institutional and other cultural differences. As Darwin (1874) argued in The Descent of Man, many human groups meet the basic criteria necessary for selection to operate: heritable variation and competition, the outcome of which is based on that heritable variation. Ethnographic evidence of the prerequisites for and operation of CGS in the simplest societies suggests that CGS has operated in our lineage for a few tens of millennia, if not longer. If so, the cooperative imperatives produced by rudimentary culturally transmitted institutions may well have shaped our innate social psychology. This is reflected by the observation that young children learn norms and act on them, but chimpanzee societies have, at best, rudimentary norms. Individual-level human decisions regarding the invention of new cultural variants, the spread of variants by diffusion, and the choices of group affiliation are likely to be strongly colored by moral intuitions like sympathy and patriotism. The current direct evidence for culture-led gene-culture coevolution is only strong for genes that do not directly affect behavior, but evidence regarding behavioral traits is likely to improve greatly in the next decade.

We submit that the evidence sketched here is more than sufficient to justify taking the CGS hypothesis seriously as a basic explanation for our species' highly unusual ability to create large societies with widespread cooperation between non-relatives. We do not see how any of the alternatives to CGS can easily account for the institutionalized cooperation that characterizes human societies. We certainly do not claim that a large body of studies has met the gold standard of accurately estimating the strength of any of the three CGS processes we have discussed. Nor have we estimated the strength of culture-based social selection on human genes. These projects are in their infancy. We have pointed to excellent pioneering studies showing that a proper quantitative investigation of cultural evolution and gene-culture coevolution is possible (e.g., Bouckaert et al. 2012; Hout et al. 2001; Itan et al. 2009). We need an empirical game theory that estimates costs and benefits generated by people playing various strategies in institutional games. Do individuals bear costs or neglect to take benefits that accrue to them as individuals in order to benefit their social groups? How large are such benefits and costs? Are organizations with many individuals who are willing to bear costs or forego benefits really more likely to win contests with other groups, or attract imitators and immigrants? How does rewarding individuals with prestige or symbolically with medals and prizes affect their genetic fitness or cultural success? The bad news is that the games people play are very complex, and many of those most relevant to human evolution took place many generations ago. The good news is that evolutionary biologists have struggled fairly successfully with similar complexities in organic evolution (Endler 1986).

\section{Appendix}

Table 1. Description of data with corresponding $\boldsymbol{F}_{S T}$ values plotted in Figure 1.

\begin{tabular}{|c|c|c|c|c|}
\hline Data description & Groups (sample size) & $\begin{array}{l}\text { Group type } \\
\text { (1=religious, } \\
2=\text { political, } \\
3=\text { ethnic, } \\
\text { 4=nations/ } \\
\text { provinces) }\end{array}$ & $F_{\mathrm{ST}}$ & Reference \\
\hline $\begin{array}{l}\text { Survey (Nov13-Nov24 2006). } \\
\text { Public opinion amidst rising } \\
\text { violence in Afghanistan. } \\
\text { Opinions on the US, Osama } \\
\text { Bin Laden, intervention by } \\
\text { NATO,... }\end{array}$ & $\begin{array}{l}\text { Pashtun (872), Tajik } \\
\quad \text { (718), Uzbek } \\
\quad(225), \text { Turkmen } \\
\quad(50), \text { Hazara (189) }\end{array}$ & 3 & 0.065 & $\begin{array}{l}\text { WPO-PIPA-“Afghan Approval of the Karzai } \\
\text { Government and Western Forces, Though } \\
\text { Still Strong, Is Declining” http://drum.lib. } \\
\text { umd.edu/handle/1903/10120 }\end{array}$ \\
\hline $\begin{array}{l}\text { Environmental attitudes in } \\
\text { California }\end{array}$ & $\begin{array}{l}\text { somewhat politically } \\
\text { liberal (473), } \\
\text { somewhat } \\
\text { conservative }(562)\end{array}$ & 2 & 0.098 & $\begin{array}{l}\text { Public Policy Institute of California, "PPIC } \\
\text { Statewide Survey: Californians and the } \\
\text { Environment" }\end{array}$ \\
\hline
\end{tabular}


Richerson et al.: Cultural group selection plays an essential role in explaining human cooperation

Table 1 (Continued)

\begin{tabular}{lll}
\hline \hline Data description & Groups (sample size) & Group type \\
& (1=religious, & \\
& $2=$ Rolitical, \\
& $3=$ ethnic, \\
& $4=$ nations/ \\
& provinces)
\end{tabular}

Environmental attitudes in California

State-level opinion on the allocation of resources by State and local governments

State-level opinion on the allocation of funds by State and local governments

Attitudes toward taxation, unions, immigrants, voting, special elections, education

2008 Survey. The role of clergy in the political system.

(1) Statement A - Religion must be respected, but clergy should not dictate the political system. (2) Statement B Clergy must play a greater role in our political system.

2008 Survey. The right of women Saudi Arabia (760), to work outside the home.

2008 Survey. The role of clergy in the political system. (1) Statement A - Religion must be respected, but clergy should not dictate the political system. (2) Statement B Clergy must play a greater role in our political system.

2008 Survey. The right of women Muslims (748), to work outside the home.

Likely scenario for peace or war Muslims (748), with Israel

June 2005 survey. Attitudes on international trade policies and the role of government among workers whose jobs were lost because of overseas trade or changing industry demand
Christians (92)

very politically liberal

(257), very

politically

conservative (338)

Caucasian or White

and Non-Hispanic

(1375), Hispanic or

Latino (638)

Born in the US

(1829), Not Born

in the US (662)

Affiliate a lot with the

Tea Party (92),

Does not affiliate

at all with the Tea

Party (426)

Saudi Arabia (760), Lebanon (600)

Lebanon (600)

Muslims (748),

Christians (92)

1

0.063

$4 \quad 0.591$

10.105

Democrat (306)
0.041

3

3

0.027

2

0.421

4

0.612
0.277
Republican (287),

\section{Christians (92)}

0.009

2

0.061
Public Policy Institute of California, "PPIC Statewide Survey: Californians and the Environment"

Public Policy Institute of California, "PPIC Statewide Survey: Californians and Their Government"

Public Policy Institute of California, "PPIC Statewide Survey: Californians and Their Government"

Field (California) Poll March 2011. San Francisco, CA: Field Research, Poll 08-05.
Shibley Telhami/Sadat Chair, University of Maryland - Professor Shibley Telhami, Principal Investigator. http://sadat.umd.edu/surveys/index.htm
Shibley Telhami/Sadat Chair, University of Maryland - Professor Shibley Telhami, Principal Investigator. http://sadat.umd.edu/surveys/index.htm Shibley Telhami/Sadat Chair, University of Maryland - Professor Shibley Telhami, Principal Investigator. http://sadat.umd.edu/surveys/index.htm

Shibley Telhami/Sadat Chair, University of Maryland - Professor Shibley Telhami, Principal Investigator. http://sadat.umd.edu/surveys/index.htm Shibley Telhami/Sadat Chair, University of Maryland - Professor Shibley Telhami, Principal Investigator. http://sadat.umd.edu/surveys/index.htm The PIPA/Knowledge Networks poll, The American Public on International Issues. 
Richerson et al.: Cultural group selection plays an essential role in explaining human cooperation

Table 1 (Continued)

\begin{tabular}{|c|c|c|c|c|}
\hline Data description & Groups (sample size) & $\begin{array}{l}\text { Group type } \\
\text { (1=religious, } \\
2=\text { political, } \\
3=\text { ethnic, } \\
4=\text { nations/ } \\
\text { provinces) }\end{array}$ & $F_{\mathrm{ST}}$ & Reference \\
\hline $\begin{array}{l}\text { July } 2004 \text {. Attitudes on the use of } \\
\text { detention and torture for the } \\
\text { war on terrorism }\end{array}$ & $\begin{array}{l}\text { Republican (126), } \\
\text { Democrat (165) }\end{array}$ & 2 & 0.152 & $\begin{array}{l}\text { The PIPA/Knowledge Networks poll, The } \\
\text { American Public on International Issues: } \\
\text { Americans on Detention, Torture, and the } \\
\text { War on Terrorism }\end{array}$ \\
\hline $\begin{array}{l}\text { September } 2007 \text {. Attitudes on } \\
\text { the further adoption of Sharia } \\
\text { law: As compared to current } \\
\text { Pakistan law, do you think that } \\
\text { Shari'a should play a larger } \\
\text { role, a smaller role, or about } \\
\text { the same role in Pakistan law } \\
\text { as it plays today? }\end{array}$ & $\begin{array}{l}\text { Pakistanis from the } \\
\text { provinces of } \\
\text { Punjab (358), Sind } \\
\text { (269), NWFP (79) } \\
\text { and Blouchistan } \\
\text { (34) }\end{array}$ & 4 & 0.041 & $\begin{array}{l}\text { WorldPublicOpinion.org, Pakistani Public } \\
\text { Opinion on Democracy, Islamic Militancy, } \\
\text { and Relations with the US; Sept. 12-28, } \\
2007\end{array}$ \\
\hline $\begin{array}{l}\text { June } 1982 \text {. Attitude toward } \\
\text { whether abortion should "be } \\
\text { left as it is, made easier to get, } \\
\text { or more difficult to get." }\end{array}$ & $\begin{array}{l}\text { Church of England } \\
\text { (653), Catholic } \\
\text { (189) }\end{array}$ & 1 & 0.060 & $\begin{array}{l}\text { Walker, N. and Marsh, C., Public Attitudes } \\
\text { towards Sentenced Offenders and } \\
\text { Abortion, } 1982 \text { [computer file]. Colchester, } \\
\text { Essex: UK Data Archive [distributor], } 1983 . \\
\text { SN: } 1898 .\end{array}$ \\
\hline $\begin{array}{l}\text { June 1982. Attitude toward } \\
\text { whether abortion should "be } \\
\text { left as it is, made easier to get, } \\
\text { or more difficult to get." }\end{array}$ & $\begin{array}{l}\text { Catholic (189), } \\
\text { Atheist/Agnostic } \\
(69)\end{array}$ & 1 & 0.146 & $\begin{array}{l}\text { Walker, N. and Marsh, C., Public Attitudes } \\
\text { towards Sentenced Offenders and } \\
\text { Abortion, } 1982 \text { [computer file]. Colchester, } \\
\text { Essex: UK Data Archive [distributor], } 1983 . \\
\text { SN: } 1898 .\end{array}$ \\
\hline $\begin{array}{l}\text { (1982). Whether the respondent } \\
\text { agrees to homosexual couples } \\
\text { living together }\end{array}$ & $\begin{array}{l}\text { Church of England } \\
\text { (644), Catholic } \\
\text { (188) }\end{array}$ & 1 & 0.018 & $\begin{array}{l}\text { Walker, N. and Marsh, C., Public Attitudes } \\
\text { towards Sentenced Offenders and } \\
\text { Abortion, } 1982 \text { [computer file]. Colchester, } \\
\text { Essex: UK Data Archive [distributor], } 1983 . \\
\text { SN: } 1898 .\end{array}$ \\
\hline $\begin{array}{l}\text { (1982). Whether the respondent } \\
\text { agrees to homosexual couples } \\
\text { living together }\end{array}$ & $\begin{array}{l}\text { Catholic (188), } \\
\text { Atheist/Agnostic } \\
(70)\end{array}$ & 1 & 0.040 & $\begin{array}{l}\text { Walker, N. and Marsh, C., Public Attitudes } \\
\text { towards Sentenced Offenders and } \\
\text { Abortion, } 1982 \text { [computer file]. Colchester, } \\
\text { Essex: UK Data Archive [distributor], } 1983 . \\
\text { SN: } 1898 .\end{array}$ \\
\hline $\begin{array}{l}\text { 2009. The respondent's opinion } \\
\text { regarding same-sex marriage } \\
\text { in California }\end{array}$ & $\begin{array}{l}\text { No preference/ } \\
\text { Atheist (99), } \\
\text { Catholic (149) }\end{array}$ & 1 & 0.021 & $\begin{array}{l}\text { Field Research Corporation, } 601 \text { California } \\
\text { Street, Suite } 900 \text { 022509, San Francisco, } \\
\text { CA 94108. Final Late February } 2009 \text { Field } \\
\text { Poll-Questionnaire }\end{array}$ \\
\hline $\begin{array}{l}\text { 2009. The respondent's opinion } \\
\text { regarding same-sex marriage } \\
\text { in California }\end{array}$ & $\begin{array}{l}\text { No preference/ } \\
\text { Atheist (99), } \\
\text { Protestant (190) }\end{array}$ & 1 & 0.131 & $\begin{array}{l}\text { Field Research Corporation, } 601 \text { California } \\
\text { Street, Suite } 900 \text { 022509, San Francisco, } \\
\text { CA 94108. Final Late February } 2009 \text { Field } \\
\text { Poll-Questionnaire }\end{array}$ \\
\hline $\begin{array}{l}\text { 2009. The respondent's opinion } \\
\text { regarding same-sex marriage } \\
\text { in California }\end{array}$ & $\begin{array}{l}\text { No preference/ } \\
\text { Atheist (99), } \\
\text { Jewish (81) Other } \\
\text { Christian }\end{array}$ & 1 & 0.179 & $\begin{array}{l}\text { Field Research Corporation, } 601 \text { California } \\
\text { Street, Suite } 900 \text { 022509, San Francisco, } \\
\text { CA 94108. Final Late February } 2009 \text { Field } \\
\text { Poll-Questionnaire }\end{array}$ \\
\hline $\begin{array}{l}\text { 2009. The respondent's opinion } \\
\text { regarding same-sex marriage } \\
\text { in California }\end{array}$ & $\begin{array}{l}\text { Hispanic (111), non- } \\
\text { Hispanic (547) }\end{array}$ & 3 & 0.021 & $\begin{array}{l}\text { Field Research Corporation, } 601 \text { California } \\
\text { Street, Suite } 900 \text { 022509, San Francisco, } \\
\text { CA 94108. Final Late February } 2009 \text { Field } \\
\text { Poll-Questionnaire }\end{array}$ \\
\hline $\begin{array}{l}\text { 2006. Opinions on conditions for } \\
\text { peace with Israel (if at all) }\end{array}$ & $\begin{array}{l}\text { Shia (145), Christian } \\
\quad(210)\end{array}$ & 1 & 0.337 & $\begin{array}{l}\text { Shibley Telhami/Sadat Chair, University of } \\
\text { Maryland - Professor Shibley Telhami, } \\
\text { Principal Investigator. } \\
\text { http://sadat.umd.edu/surveys/index.htm }\end{array}$ \\
\hline
\end{tabular}


Commentary/Richerson et al.: Cultural group selection plays an essential role in explaining human cooperation

Table 1 (Continued)

\begin{tabular}{|c|c|c|c|c|}
\hline Data description & Groups (sample size) & $\begin{array}{l}\text { Group type } \\
\text { (1=religious, } \\
2=\text { political, } \\
3=\text { ethnic, } \\
4=\text { nations/ } \\
\text { provinces) }\end{array}$ & $F_{\mathrm{ST}}$ & Reference \\
\hline
\end{tabular}

2006. Opinions on conditions for Shia (145), Druze

peace with Israel (if at all)

(27)

2006. Opinions on conditions for Shia (145), Sunni

(143)

peace with Israel (if at all)

2009. Opinions on good State governance in California

Conservative (314), Liberal (204)

2003. Georgia Poll. Do you favor or oppose government

Democrat (128), Republican (128)

involvement in health care?

2003. Georgia Poll. Do you favor Democrat (128), or oppose the U.S. Republican (128)

1

0.568

Shibley Telhami/Sadat Chair, University of Maryland - Professor Shibley Telhami, Principal Investigator.

http://sadat.umd.edu/surveys/index.htm

Shibley Telhami/Sadat Chair, University of Maryland - Professor Shibley Telhami, Principal Investigator. http://sadat.umd.edu/surveys/index.htm

Field Research Corporation, 601 California Street, Suite 900 100209, San Francisco, CA 94108. Final Late September/Early October 2009 Field Poll

Fall Georgia Poll, 2003, Survey Research Center, The University of Georgia, Athens, GA 30602 - James J. Bason, Director

Fall Georgia Poll, 2003, Survey Research Center, The University of Georgia, Athens, GA 30602 - James J. Bason, Director

government spending $\$ 15$

billion dollars on the AIDs epidemic in Africa?

2003. Georgia Poll. I worry that Democrat (140), pesticides on the foods I buy will seriously harm me or my family. Agree/Disagree
Republican (149)
Fall Georgia Poll, 2003, Survey Research Center, The University of Georgia, Athens, GA 30602 - James J. Bason, Director

\section{Open Peer Commentary}

\section{Testing the cultural group selection hypothesis in Northern Ghana and Oaxaca}

doi:10.1017/S0140525X15000047, e31

\section{Cristina Acedo-Carmona and Antoni Gomila \\ Human Evolution and Cognition, University of the Balearic Islands, Campus Carretera Valldemossa, 07122 Palma de Mallorca, Spain. \\ cacedo33@hotmail.com toni.gomila@uib.cat \\ www.evocog.org antonigomila.wordpress.com}

Abstract: We examine the cultural group selection (CGS) hypothesis in light of our fieldwork in Northern Ghana and Oaxaca, highly multiethnic regions. Our evidence fails to corroborate two central predictions of the hypothesis: that the cultural group is the unit of evolution, and that cultural homogenization is to be expected as the outcome of a selective process.

While we agree with Richerson et al. that prosocial emotions play a central role in fostering group cohesion (Acedo-Carmona \& Gomila 2013; 2014b), we disagree with them in their lack of enough attention to the internal complexity within social groups. Such internal structure, though, casts doubt on the role that group competition is required to play according to the cultural group selection (CGS) hypothesis, as well as on the role culture is assigned. Two arguments are developed to articulate this point, supported by fieldwork evidence from Northern Ghana and Oaxaca (México). These are regions characterized by the coexistence of multi-ethnic, multicultural groups and are therefore well-suited to put the predictions of CGS hypothesis to the test, which fail to get corroborated.

Argument 1. The CGS hypothesis contends that the cultural group is the relevant unit of selection for the evolution of cooperation. It follows from this that members of a group should cooperate equally with any member of the group, beyond kin. Instead, what we found in our fieldwork is that individuals within a group preferentially cooperate with the members of their respective "trust circles," and that these trust circles are small groups related to a past history of reciprocation and affiliation (Acedo-Carmona \& Gomila 2015). The cultural group is a much bigger unit, whose degree of internal cohesion derives from the topology of connections among these small groups (Acedo-Carmona \& Gomila 2014b).

This suggests that cultural groups are not the basic unit of selection of human societies - rather, small groups are, for the following reasons:

a. Even in contexts where no common culture exists, individuals develop social ties (Zhou et al. 2005) that make cooperation possible, such as trust relationships.

b. The social brain is ready to develop close relationships (Roberts et al. 2009). So, it is reasonable to think that psychological dispositions to create and maintain bonds in their immediate 
environment were selected (Henzi et al. 2007; Stiller \& Dunbar 2007), before competition among cultural groups can even start.

c. Evidence from Northern Ghana also suggests that small groups are more resistant and flexible at a time to face to hard challenges (Acedo-Carmona \& Gomila 2014a).

d. Even in contemporary societies, small groups continue playing a central role of structuring cooperation within the society (sociologists call this phenomenon "social capital"; Putnam 1993).

Selective processes at the cultural group level, therefore, cannot be basic, but dependent upon their respective internal structure of several groups, including kin-based groups and affiliation groups, with different degrees of internal cohesion and interconnection. For example, sometimes cultural groups merge into bigger ones, and sometimes groups split: Competition among groups may have little to do with these processes, but rather with internal processes at the small-group level.

Argument 2. The CGS hypothesis predicts regional cultural homogenization in the long run: If there is competition among co-local cultural groups, the successful one is deemed to prevail according to CGS, either by exclusion of the other, or by absorption/assimilation (by imitation of the most successful one, for instance). However, this is not what happened, either in Northern Ghana or in Oaxaca - on the contrary, both regions are inhabited by a plurality of cultural groups, which coexist and also cooperate, while keeping their distinctive ethnic identities (Acedo-Carmona $\&$ Gomila, 2015). In both cases, there are different origins, a settlement period, and a long history of interaction among the groups within the same region, while linguistic and cultural diversity continues, despite the fact that some ethnic groups do better than others in terms of status and welfare.

This suggests that the role of culture may have more to do with reinforcing group cohesion than promoting group competition. A shared culture may facilitate cooperation by inducing a positive expectation from individuals whose identities and values are shared (Tanis \& Postmes 2005). But in a non-instrumental way, it is not something one can choose. Culture migrations do not take place strategically because individuals by nature do not change their group identity (Bhugra \& Becker 2005). The affective sense of familiarity and belonging provided by cultural identity is too strong to allow an easy detachment toward another more advantageous culture. On the other hand, slow cultural changes take place even in successful cultural groups.

The CGS hypothesis emphasizes the well-known in-group/outgroup bias (Chen \& Xin Li 2009). However, the in-group bias that contributes to foster internal cohesion does not need to involve aversion to other groups (Mäs \& Dijkstra 2014). This aversion emerges only in cases of competition for resources (Foley \& Gamble 2009). From this point of view, cultural differentiation appears as the by-product of an adaptive process to a specific environment by a society (Alvard 2003), rather than a way to compete with other social groups in the first place, while keeping track of the basic forms of social cohesion (such as trust). This approach also may account for the diversity of existing cultures, even in the same territory: The prediction is that each society will take advantage of some of the opportunities of the environment, such as peasants versus shepherds or merchants versus state officials, as it happens in Northern Ghana (Acedo-Carmona \& Gomila 2014a). Competition derived from population explosion is a too recent phenomenon to have played an evolutionary role.

In summary, in our view, the cement of society is to be found in the basic human disposition to trust each other, which gives rise to small groups. Societies are constituted by these groups, and their interrelationships are different. Culture certainly molds these interrelationships and organization, according to tradition and environmental conditions. But its role in fostering cooperation does not seem to be primarily in providing a competitive advantage, as claimed by CGS.

\section{Cultural evolution need not imply group selection}

\author{
doi:10.1017/S0140525X15000059, e32
}

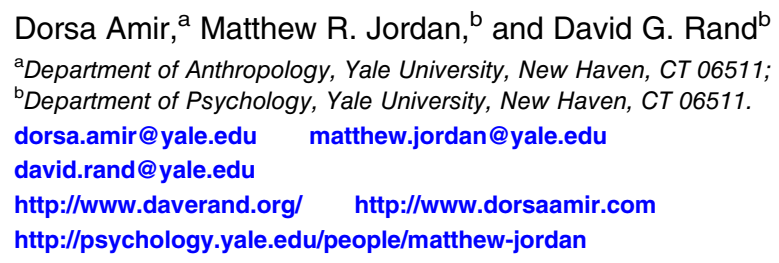

Abstract: Richerson et al. make a compelling case for cultural evolution. In focusing on cultural group selection, however, they neglect important individual-level accounts of cultural evolution. While scientific discourse typically links cultural evolution to group selection and genetic evolution to individual selection, this association is due to historical accident only. We thus call for more consideration of individual-level cultural evolution.

The target article provides compelling evidence that culture is a real phenomenon that varies across groups and evolves over time. The authors go on to argue that cultural evolution, with selection occurring at the level of the group, is necessary for explaining human cooperation. In doing so, however, they overlook the importance of individual-level cultural evolution.

Cultural evolution need not be focused on group-level selection. As long as variation exists in ideas (or strategies, in a gametheoretic context), and ideas or strategies that are more successful are more likely to be adopted by others, then cultural evolution is occurring. From a mathematical perspective, such a process is formally equivalent to (individual-level) genetic evolution, and it can explain both interpersonal cooperation and the emergence of institutions that govern human behavior.

With respect to human cooperation, consider the theoretical perspective offered by the social heuristics hypothesis ( $\mathrm{SHH}$ ), which combines cultural evolution with dual-process models of cognition. The $\mathrm{SHH}$ posits that individuals internalize strategies that are typically advantageous in daily life (Rand et al. 2014b) and carry these automatic and intuitive responses with them into atypical social situations (such as laboratory experiments). Therefore, imitation and learning, as shaped by one's physical and cultural environment, determine an individual's internalized norms and preferences. Such a process can lead to substantial variation in norms across groups (as different groups inhabit different environments) and support the evolution of cooperation in one-shot anonymous settings (via spillovers from the cooperation that is typically advantageous due to reciprocity, reputation, and punishment).

Empirical support for the SHH comes from evidence that experimentally inducing greater intuitive thinking promotes cooperation in one-shot anonymous economic games relative to inducing deliberation: Applying time constraints (Cone \& Rand 2014; Rand et al. 2012; Rand \& Kraft-Todd 2014; Rand et al. 2014a; 2014b), conceptually priming intuition (Lotz 2014; Rand et al. 2012), inducing cognitive load (Cornelissen et al. 2011; Roch et al. 2000; Schulz et al. 2014), or increasing the immediacy of payments (Dreber et al. 2014; Kovarik 2009) can increase participants' willingness to pay costs to provide benefits to strangers. Furthermore, cultural factors, such as trust of daily-life interaction partners, alter participants' intuitive responses as predicted by such a theory: Intuition only promotes cooperation among those who come from a culture of cooperation and trust, where cooperation is thus advantageous (Capraro \& Cococcioni 2015; Rand \& Kraft-Todd 2014). Direct evidence of the spillover effects that are at the heart of the SHH comes from laboratory experiments where participants are randomly assigned to interact under rules that make cooperation or defection advantageous: Participants experiencing a social environment that favors defection are dramatically less prosocial in subsequent one-shot anonymous interactions, but only if they rely on intuitive, heuristic processing (Peysakhovich \& Rand 2015). 
This theory accommodates the innate learning structures used in norm acquisition (Hamlin et al. 2011), while also explaining cross-cultural differences in norms and preferences (Ellingsen et al. 2012; Gächter et al. 2010; Henrich et al. 2001; 2010b; Herrmann et al. 2008). Importantly, the SHH accomplishes this without an appeal to group selection or intergroup conflict. An individual benefits from internalizing successful strategies, and in typical settings where mechanisms for the evolution of cooperation are operating, what is good for the individual winds up also being beneficial for the group. Thus, improving the group's welfare occurs as a side effect of selection maximizing individual payoffs, rather than as the cause of that selection.

What about institutions? By enforcing norms of cooperation, institutions play a critical role in stabilizing payoff structures that simultaneously optimize social and individual welfare. Can the emergence of such institutions be explained by individual-level forces? Indeed, it can. Consider the institution of democratic voting. Under a median voting rule, for instance, individuals engaged in a group cooperation endeavor each vote for a contribution amount, and then all participants are forced to contribute the amount specified by the median voter (Bernard et al. 2013; Deacon \& Shapiro 1975; Hauser et al. 2014; Walker et al. 2000). Under such an institution, even self-interested people would vote for contribution to a public good, because individuals earn higher payoffs in a group where everyone contributes. Since the institution forces all participants to behave in the same way, the free-rider problem is eliminated, and individual and collective interests are aligned. Furthermore, similar logic would cause individuallevel forces to favor joining or founding such an institution, rather than preferring a scenario where free-riding was possible (and thus cooperation was doomed). Indeed, experiments show that people "vote with their feet" and learn to choose institutions with sanctions over a sanction-free alternative (Gürerk et al. 2006; Rockenbach \& Milinski 2006). These studies capture the essential components of institutionalized cooperation: When institutions can homogenize individual behavior through norm enforcement, social welfare and individual welfare are maximized by the same strategy.

Thus, cultural evolution and learning need not be linked to grouplevel selection, and genetic evolution to individual-level selection, as is done in the target article (and in much of the literature on cultural evolution more broadly). Both of these dimensions of the debate regarding human evolution can vary independently (Rand \& Nowak 2013). A helpful analogy comes from American politics, where social and fiscal conservatism, two independent dimensions of political attitudes, tend to strongly co-vary because of the United States' two-party system. However, these two variables do not need to covary: An individual can be socially liberal and fiscally conservative (e.g., libertarian) or socially conservative and fiscally liberal. Similarly, scholars can advocate for the importance of cultural evolution without invoking group selection, an intellectual space which is often left unoccupied. It is largely due to historical accident that cultural evolution and group selection have come to be linked (the most successful proponents of the importance of culture are also advocates of group selection). Continuing to develop individual-level accounts of cultural evolution is essential for deepening our understanding of human evolution and prosociality.

\section{The burden of proof for a cultural group selection account}

\author{
doi:10.1017/S0140525X15000060, e33
}

\author{
Pat Barclay ${ }^{\mathrm{a}}$ and Daniel Brian Krupp ${ }^{\mathrm{b}, \mathrm{c}}$ \\ ${ }^{a}$ Department of Psychology, University of Guelph, Guelph, ON N1G 2W1, \\ Canada; ${ }^{\mathrm{b}}$ Program in Evolution and Governance, One Earth Future, \\ Broomfield, CO 80021; ' Department of Psychology, Queen's University, \\ Kingston, ON K7L 3N6, Canada. \\ barclayp@uoguelph.ca dbkrupp@saltlab.org \\ www.patbarclay.com http://www.saltlab.org
}

Abstract: Richerson et al. establish cultural group selection as a plausible force in human social evolution. However, they do not demonstrate its causal precedence for any trait, let alone its "essentialness." To do so, they must show that a particular group trait was caused by cultural transmission, and directly caused differences in group fitness.

Richerson et al. have done a tremendous job of establishing the plausibility of cultural group selection (CGS) as a process of social evolution. However, they have not shown that it is "essential" to human cooperation, or even that it has operated on any single trait. Instead, they show that fitness can be partitioned so as to satisfy the conditions for CGS. This is an altogether different - and much weaker - claim.

The total fitness of a population can be carved up in multiple ways and attributed to any number of traits, so long as fitness and trait values covary. This is one of the more pleasing results derived from the Price equation (e.g., Price 1972; see also Hamilton 1975; Marshall 2011; Queller 1992), and it should bury the notion that individual- and multi-level selection accounts are predictively different. An allele that is favored in inclusive fitness or neighbor-modulated models will also be favored in group selection models (and vice versa), because the frequency of that allele is positively correlated with fitness whether we conceive of it as affecting individuals or groups. If CGS favors the same fitness effects as selection on genes (not a given, of course), then the findings in the target article should not be news. The average fitness of individuals is necessarily correlated with the fitness of their groups.

But just because we could view selection as taking place at the group level does not mean we should. Although individual-level and multi-level fitness partitions are predictively equivalent, they are not causally so (Birch \& Okasha 2015; Okasha 2015; see also Okasha \& Paternotte 2012). Sometimes, group traits directly cause group fitness, but at other times the relationship between the two is merely correlational. Using the wrong fitness partition will lead one to infer causality where none exists and, consequently, mischaracterize the adaptation. If there is individual-level variation that directly causes variation in individual fitness (Fig. 1A), then an individual-level partition is best. Of course, individual variation in traits will also directly affect trait variation at the group level; this is what Williams (1966) meant in saying that "a herd of fleet deer" will simultaneously appear as a "fleet herd." In this instance, the target of selection is the fleetness of individuals, rather than that of the groups they make up. Conversely, trait variance at the group
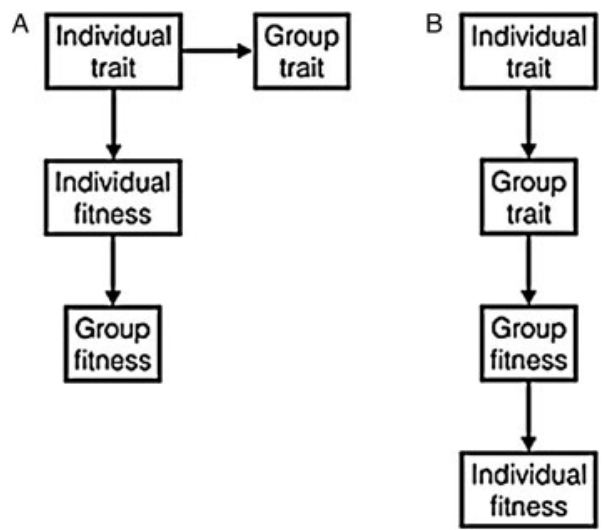

Figure 1 (Barclay \& Krupp). Causality in individual and group selection. Arrows show the direction of direct causal effects. (A) An individual-level partition is justified when the individual trait directly affects individual fitness and there is no direct relationship between the group trait and group fitness. This results in an individual adaptation. (B) A group-level partition is justified when only the group trait directly affects group fitness and there is no direct relationship between the individual trait and individual fitness. This results in a group adaptation. Adapted from Okasha (2015). 
Commentary/Richerson et al.: Cultural group selection plays an essential role in explaining human cooperation

level that directly causes variance in group fitness (Fig. 1B) is best handled by a group- or multi-level partition.

Consider warfare as an illustration. In typical battles where some live and die on each side, individual fitnesses cause group fitness through simple summation. In fights where the loser is annihilated (e.g., the losing battleship is sunk), however, individual fitness is directly caused by group fitness-individuals succeed or fail because their group succeeds or fails. A multi-level partition, such as CGS, is only warranted in the latter sort of case.

The burden of proof for a CGS account, then, is considerably heavier than what the target article presents. First, CGS needs to be tested on a case-by-case basis; it cannot be accepted wholesale because it cannot be distinguished from alternative fitness partitions in this way. A hypothesis for a particular group trait must be set up against alternative hypotheses. Second, the evidence must demonstrate the direct causality of the link between the group trait and group fitness (Fig. 1B), beyond mere correlation. Third, the evidence must show that the group trait has evolved and is maintained specifically by cultural transmission. While Richerson et al. do not ignore these issues, their "sketch of the evidence" falls short of making their case compelling.

Many alleged examples of group selection likely reflect individual-level adaptations. The requirements for group, as opposed to individual, adaptation are rather imposing: It is rare for individual human fates to be so intertwined with their group's fate that group success directly causes (rather than merely correlates with) individual success, such as all group members succeeding or failing together as a unit because of their group's composition. Generally, we can expect group traits to directly cause group adaptation only when partners are clonal or when there is extreme repression of competition (Gardner \& Grafen 2009; Okasha \& Paternotte 2012), neither of which describes the human condition well. The examples given by Richerson et al. do not come close to meeting this criterion. Conflicts that lead to the success or failure of entire groups would, but such circumstances are rare.

Moreover, many cases of ostensible cultural transmission are explainable as strategic, individual responses to existing socio-ecological circumstances. Violent defense of one's honor - a key aspect of the "Culture of Honor" - in the Southern United States is often given as a shining example of cultural transmission (e.g., Nisbett $\&$ Cohen 1996; Richerson \& Boyd 2005). However, current variation in income inequality fully accounts for any cultural difference in homicide rates between the northern and southern states (Daly \& Wilson 2010). Similarly, behavioral differences between collectivist and individualist cultures are supposedly maintained by internalized social norms and beliefs (e.g., Markus \& Kitayama 1991), yet these differences can disappear when the expectations of the different cultural groups are matched (Yamagishi et al. 2008).

Richerson et al. wave away alternative explanations by toppling a straw-man of "evoked culture" that they readily admit no one holds and by relegating as "narrow" the interpretation of experimental research. They also ignore the fact that CGS explains genetically detrimental helping by invoking the "mistakes" of an adaptive learning mechanism (Barclay \& Van Vugt 2015). More generally, the study of cooperation has long been hindered by confusion between proximate and ultimate causation (Barclay 2012; West et al. 2011), and we can now add to the list a persistent confusion over the utility of the group selection concept itself. We look forward to future work on CGS that convincingly demonstrates both the causal relationship between group traits and group fitness and the transmission of these traits by cultural means.

\section{The cooperative breeding perspective helps in pinning down when uniquely human evolutionary processes are necessary}

\author{
doi:10.1017/S0140525X15000072, e34
}

Judith Maria Burkart and Carel P. van Schaik
Anthropological Institute and Museum, University of Zurich.
Judith.Burkart@aim.uzh.ch vschaik@aim.uzh.ch
www.aim.uzh.ch

Abstract: The cultural group selection (CGS) approach provides a compelling explanation for recent changes in human societies, but has trouble explaining why our ancestors, rather than any other great ape, evolved into a hyper-cooperative niche. The cooperative breeding hypothesis can plug this gap and thus complement CGS, because recent comparative evidence suggests that it promoted proactive prosociality, social transmission, and communication in Pleistocene hominins.

Richerson et al. address two key changes in human evolution, the first being how cooperation could evolve in the small-scale Pleistocene societies of prehistoric times, and the second being how these small-scale societies successfully evolved into much larger and more complex societies during the Holocene. The authors' case for a role of cultural group selection (CGS) in the second transition is strong. However, we will argue that the adoption of cooperative breeding suffices to explain the origin of human hyper-cooperation in early forager societies, as it resulted in increased prosociality and social transmission and favored the emergence of language.

Richerson et al. mention cooperative breeding as a possible trigger of the process involving CGS, but argue that this alternative hypothesis is difficult to test independently. However, recent comparative work exploring the psychological and cognitive consequences of cooperative breeding in nonhuman primates now increasingly allows us to identify general patterns that reliably emerge whenever a primate species adopts cooperative breeding. It is thus most parsimonious to assume that such psychological and
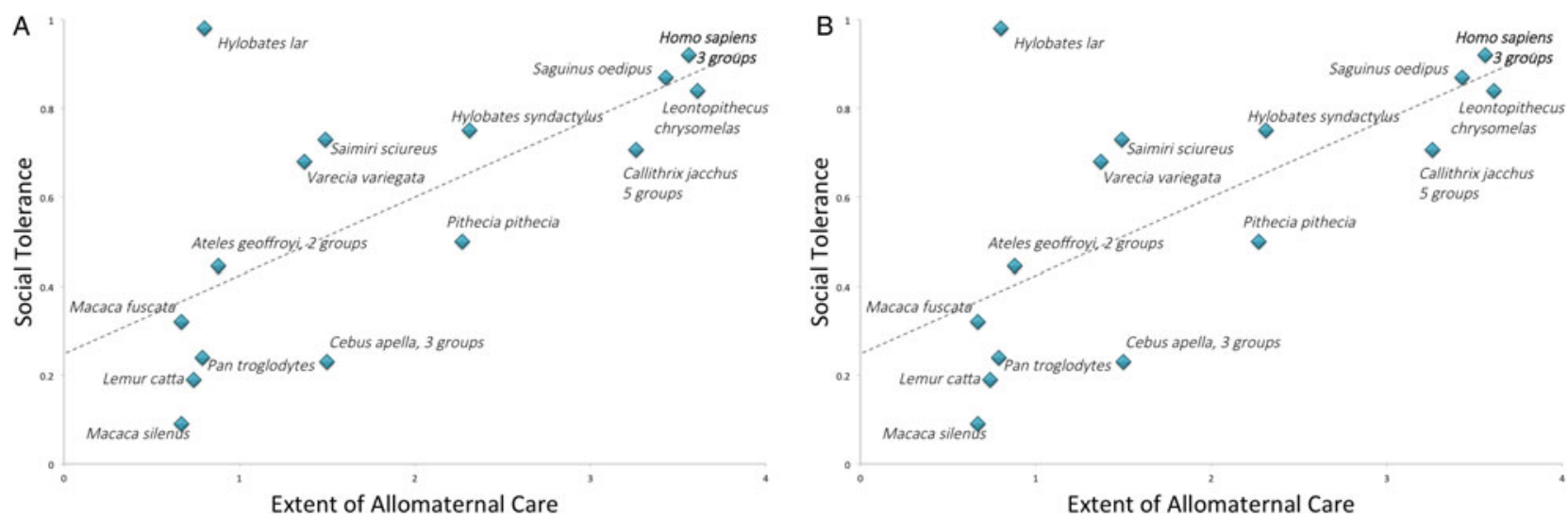

Figure 1 (Burkart \& van Schaik). The relationship between allomaternal care and (A) proactive prosociality and (B) social tolerance. Humans fit the general primate trend and do not represent an outlier. 
cognitive consequences also arose when our hominin ancestors, but none of the other great apes, started to engage in cooperative breeding (Hrdy 2009; Kramer 2010). Together, these consequences are likely to have paved the way for the emergence of CGS processes (van Schaik \& Burkart 2010).

Proactive prosociality. The first and perhaps most important consequence of cooperative breeding is that it leads to higher levels of proactive prosociality at the group level. To test this, we used the group service paradigm, in which an individual can provide food to other group members without receiving any food itself, and applied it to 24 groups of 15 species of nonhuman primates (Burkart et al. 2014). The results (Fig. 1A) show that the extent of allomaternal care directed at immatures is the best predictor of experimentally assessed proactive prosociality within a group, far better in fact than any other factor proposed so far, including brain size, social tolerance, cooperative hunting, or the presence of strong social bonds. Most importantly, humans perfectly fit the nonhuman primate regression, suggesting that we do not need a special explanation for our proactive prosociality.

Social transmission. Cooperatively breeding primates, the callitrichid monkeys, consistently show enhanced performance in social learning tasks (Burkart \& van Schaik 2010; Snowdon 2001) compared to their independently breeding sister taxa, most likely due to their high social tolerance (Coussi-Korbel \& Fragaszy 1995), which, like proactive prosociality, demonstrably increases with increasing levels of allomaternal care across primates (Fig. 1B). Indeed, callitrichid monkeys not only show high levels of social learning, but also the best evidence for teaching among nonhuman primates comes from callitrichids (Dell'Mour et al. 2009; Humle \& Snowdon 2008; Rapaport 2011), and not from the intelligent, yet independently breeding great apes (Boesch 1991; Humle et al. 2009; Lonsdorf 2006). Among non-primates, teaching is similarly over-represented in cooperatively breeding species (Byrne \& Rapaport 2011; Kline 2015).

Communication and language. Callitrichids also show remarkably complex vocal communication (Rukstalis et al. 2003; Zuberbühler 2012), with large vocal repertoires and unusual levels of vocal learning, both as immatures (including babbling) and as adults, as well as vocal control (Roy et al. 2011; Snowdon 2013). Intriguingly, and reflecting their prosocial attitude, cooperatively breeding monkeys regularly use food-offering calls (Vitale et al. 2003) and cooperative vocal communication (Takahashi et al. 2013), in that they take turns in extended sequences of call exchanges that are based on conversation rules strikingly similar to those used by humans (Stivers et al. 2009). Notably, apes altogether lack communicative turn taking. Information donation (in the form of functionally referential food calls, teaching, or vocal cooperation; Takahashi et al. 2013) is thus over-represented in cooperatively breeding primates compared to independently breeding ones. It is the basis for language, which explains why apes, who possess most basic cognitive requirements for language but largely lack this prosocial motivation (Tomasello 2007), never evolved language.

These broad evolutionary trends can explain why human psychology became different from that of the other great apes: Alone among great apes, our ancestors started to rear their offspring cooperatively. Extensive allomaternal care is a better explanation than the other source of human cooperation, male bonding and the resulting cooperative hunting and indirect reciprocity in food sharing, because in other male-bonded or cooperatively hunting species, we do not see similar prosocial tendencies, unless they also are cooperative breeders (Burkart et al. 2009; Burkart \& van Schaik 2010).

In sum, comparative evidence supports the idea that cooperative breeding installed in our ancestors the psychological predispositions that functioned as the pre-adaptive substrate upon which CGS could eventually be built: the design for group function (language - see target article, sect. 6.1) and mechanisms maintaining intergroup variation (social learning - sect. 2 ; prosociality-sect. 6.3). This argument enhances the plausibility of
CGS in the Holocene transition. First, without this foundation, CGS has a circularity problem. High-fidelity, low-cost signaling had to evolve first in order to allow gene-culture coevolutionary processes to install a more prosocial psychology, which in turn would have paved the way for high-fidelity, low-cost signaling and eventually language. Second, the adoption of cooperative breeding offers a plausible explanation for why our ancestors, but none of the other great apes, took this extraordinary trajectory. Thus, regardless of whether CGS is needed to explain the Holocene transition to large-scale societies, cooperative breeding is necessary to explain its operation in the first place.

\section{Mother-infant cultural group selection}

\section{doi:10.1017/S0140525X15000084, e35}

\section{James S. Chisholm, ${ }^{a}$ David A. Coall, ${ }^{b, c}$ and Leslie Atkinson ${ }^{d}$ ${ }^{a}$ School of Anatomy, Physiology, and Human Biology, University of Western

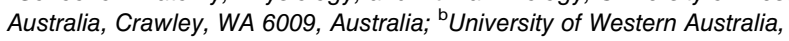 School of Psychiatry and Clinical Neurosciences, Crawley, WA 6009, Australia; 'School of Medical Sciences, Edith Cowan University, Joondalup, WA 6027, Australia; ${ }^{\mathrm{d} D e p a r t m e n t ~ o f ~ P s y c h o l o g y, ~ R y e r s o n ~ U n i v e r s i t y, ~ T o r o n t o, ~}$ ON M5B 2K3, Canada. \\ jchisholm@anhb.uwa.edu.au \\ http://www.uwa.edu.au/people/jim.chisholm \\ d.coall@ecu.edu.au \\ http://www.ecu.edu.au/schools/medical-sciences/staff/profiles/senior- \\ lecturers/dr-david-coall \\ atkinson@psych.ryerson.ca \\ http://www.ryerson.ca/psychology/faculty/atkinson/}

Abstract: Richerson et al. argue that "cultural group selection plays an essential role in explaining human cooperation." We believe that cooperation came first, making culture and thus cultural group selection possible. Cooperation and culture began - and begins - in mother-infant interaction.

Richerson et al. "do not see how any of the alternatives to CGS can easily account for the institutionalized cooperation that characterizes human societies" (target article, sect. 7, para. 2). While they make a solid case for the role of cultural group selection (CGS) in human evolution and history, we think it is the evolution of human cooperation that made the tribal social instincts and CGS possible in the first place, and that this occurred much earlier than the authors think. To explain, we refer to Weingarten and Chisholm's (2009) use of Bowlby's (1946) insight that "the psychological problem of ensuring persistent co-operative behaviour" (p. 62) in groups was solved by the evolution of the capacity of infants to develop, first, an attachment to (a powerful emotional valuation of) mothers and others, then, as they mature, to groups of others, group leaders, and group "policy" (i.e., belief/value system; Bowlby made specific reference to the cohesion, cooperation, and altruism of combat units in WWII).

From the perspective of attachment theory - and infants everyone's first group, tribe, society, institution, ethnolinguistic unit, and culture is the mother-infant group. (By mother we mean "mother and/or others who act like mothers.") Everyone's first experience of cooperation is in the resolution (or not) of parent-offspring conflict (Chisholm 2003). Because selection operates only on phenotypes, and phenotypes must develop before they are exposed to selection, we are skeptical of models of the evolution of cooperation that attribute fitness benefits to adults only. How did our adult ancestors learn to cooperate? What motivated them to cooperate?

Richerson et al. have little use for what they call "trigger hypotheses" for the evolution of cooperation because they are difficult to test and "even the most innatist theories [of language, for example] imagine a culture-led gene-culture coevolutionary process." We have little use for them because they are all about the behavior of adults-but do imagine a mother-infant culture-led 
coevolutionary process that set the stage for CGS. Like Richerson et al., we do not think that kin selection and reciprocity are sufficient to explain large-scale cooperation, but again, think they can explain where our cooperative motivations came from and how they were exposed to selection - because many of the "mechanisms that maintain intergroup variation" are already part of the attachment process. To wit, infants are capable of accurate, rapid, social learning; infants conform their behavior to their mothers' behavior; the infant-mother group is a moral community, based on trust (security) and norms of interaction, and infants and mothers punish each other for "deviant" behavior with resistance, displays of anger, withdrawal, and so forth; infants learn disproportionately from their "prestigious," all-powerful, all-resources-controlling mothers; and infants engage in "dances and similar motor rituals" with their mothers (Beebe \& Lachmann 2014; Trevarthen 2007; Tronick 2007). Moreover, oxytocin and subcortical brain systems subserve both infant and adult attachment behavior (Lane et al. 2013; Nelson \& Panksepp 1998; Schore 2013; Strathearn 2011) and trust in experimental games played by adults (Baumgartner et al. 2008; Kosfeld et al. 2005). The basic mammalian social emotions (Panksepp 2011) provide the utility function-emotional costs and benefits-guiding human choice, by infants in the attachment process and adults in cooperation with each other.

Because there is no point in thinking unless one already has something of value to think about, the basic mammalian, "bottom-up," subcortical emotions have also been implicated in the development of "top-down," neocortical thinking (Damasio 1994; 1999), of which theory mind is an early example. Theory of mind - and related constructs like intersubjectivity (Trevarthen 2011) and "time travel" (Suddendorf 2013)-enables infants to learn through (not just from) mothers by inferring (thinking about) their mothers' beliefs, desires, and intentions/actions in order to "choose" their own next action on the basis of their innate value system, the basic mammalian social emotions. Theory of mind is the beginning of culture - shared or "we" understanding/intentionality (Tomasello et al. 2005). It also varies as a function of attachment security (Fonagy \& Target 1997; Fonagy et al. 2007; Meins et al. 2002). As Hobson (2004, p. 94) put it, "symbolizing, language and thought are possible only because of the nature of the emotional connection between one person and another, and because of each person's involvement with a shared world." The cultural origin of human cognition is in the co-construction of meaning by mother and infant. We therefore partly disagree with Richerson et al.'s statement that "Perhaps the earliest cultural norms merely solidified the bonds of kinship and reciprocity that were evolving through participation in systems of cooperative breeding" (sect. 2.2, para. 2). (Again, how did kin and cooperative breeders learn to cooperate? What made them want to?) We think the earliest cultural norms evolved through mother-infant cooperation.

This brings us to "docility," one of Richerson et al.'s "trigger hypotheses": "Docile individuals more inclined to conform to norms would find it easier to enter larger, more norm-bound groups" (sect. 2.2, para. 3). If "docile" means less fearful and aggressive, we entirely agree, for two reasons. First, secure attachment is associated with less fear and aggression (Cassidy 2008; Fearon et al. 2010). Second, the Russian farm fox studies (Belyaev et al. 1985; Trut 1999) show that artificial selection for "tameness" (reduced fear/aggression) succeeded, not because "genes for" tameness were selected, but because of the delayed development of fearful/ aggressive behavior in tame strains compared to non-selected, wild strains. As a correlated by-product, the "docile" strains also showed increased social-cognitive skills (Hare et al. 2005). Weingarten and Chisholm (2009) proposed that the evolution of delayed development in humans might have had the same effect, potentially explaining how human adults came to be (relatively) docile.

In conclusion, we believe that the tribal social instincts and CGS were important very early in human evolution as our infant ancestors evolved the enhanced social-cognitive skills (theory of mind) that enabled them to "bargain and negotiate" their way through parent-offspring conflict. The outcome, everyone's first culture-mother-infant culture-repeated in every mother-infant group for hundreds of thousands of years, resulted in culture-led, gene-culture coevolution, and CGS. We are thus inclined to agree with Ayala (2010): Complex cumulative culture may well be an exaptation.

\section{Intergroup competition may not be needed for shaping group cooperation and cultural group selection}

\author{
doi:10.1017/S0140525X15000096, e36
}

\section{Carsten K. W. De Dreu a and Daniel Balliet ${ }^{\mathrm{b}}$ \\ ${ }^{\mathrm{a} D e p a r t m e n t ~ o f ~ P s y c h o l o g y, ~ a n d ~ C e n t e r ~ f o r ~ E x p e r i m e n t a l ~ E c o n o m i c s ~ a n d ~}$ Political Decision Making (CREED), University of Amsterdam, Weesperplein 4, 1018 XA, Amsterdam, The Netherlands; ${ }^{\mathrm{b}}$ Department of Social and Organizational Psychology, VU University Amsterdam, 1081 BT, Amsterdam, The Netherlands. \\ c.k.w.dedreu@uva.nl_d.p.balliet@vu.nl}

Abstract: Because intergroup interactions often are mixed-motive rather than strictly zero-sum, groups often negotiate settlements that enable both cultures to thrive. Moreover, group prosperity rests on in-group love (rather than out-group hate) that emerges also absent intergroup competition or comparison. It follows that cultural group selection (CGS) reflects group effectiveness in organizing in-group trust and cooperation, rather than winning (in)direct intergroup competitions.

Richerson et al. have identified five conditions for cultural group selection (CGS) to be considered as a viable possibility to explain cultural variation across groups and societies. One such condition is intergroup competition: "while modern nations and the subgroups ... differ in many ways from ancestral tribes, they rest on the innate foundations that evolved from competition between ethnolinguistic units" (sect. 2, para. 2), and "the success and failure of groups in such competitions is often determined by institutional and other cultural differences" (sect. 7, para. 1). The authors reason that intergroup competition for scarce resources such as cattle and territory, market share, or religious supporters motivates within-group cooperation, and creates cultural practices and culture-specific institutions. Intergroup competition allows some tribes, firms, or religions to survive and prosper, while others are marginalized. Ultimately, cultural norms and institutions are thought to provide selection pressures for genetic adaptations for social behavior, including parochial altruism.

Here we question the importance of intergroup competition in CGS and culture-led genetic evolution of adaptations for prosocial behavior. First, we take issue with the tendency in evolutionary theory in general, and in CGS in particular, to conceptualize intergroup competition as a one-dimensional and zero-sum game. Similar to inter-individual interactions (Deutsch 1973; Schelling 1960), intergroup competition can vary in the degree of corresponding versus non-corresponding interests (Bornstein 2003). In fact, CGS relies on variable-sum Prisoner's Dilemma game and Ultimatum Bargaining games when testing (parts of) CGS theory (Bowles \& Gintis 2011; Hagen \& Hammerstein 2006; Henrich 2004). Furthermore, intergroup competition often is multi-dimensional so that losses on one issue can be, and often are, offset by gains on another issue (Aaldering \& De Dreu 2012; De Dreu \& Carnevale 2003). Accordingly, there are many alternative solutions to intergroup competition than "winnertakes-all," including leaving the scene (withdrawal from the battlefield, entering a different market), or negotiating a settlement (De Dreu 2010; Pruitt \& Rubin 1986). Indeed, tribes and small-scale societies not only raid but also trade (Horan et al. 2005), firms merge much more often through negotiated deals than through hostile take-overs (Martynova \& Renneboog 2008), and disputes 
between countries may involve some hostilities and barbed-wired fences, but rarely lead to warfare (Jones et al. 1996). Thus, intergroup interactions often involve some corresponding interests and can and are settled peacefully through negotiated deals that allow both sides, and their respective cultures, to survive and prosper. As such, it is difficult to see whether and how intergroup competition is, or has been, a powerful selective pressure on the evolution of group culture, and hence, culture-led genetic evolution.

Second, Richerson et al. suggest that this intergroup competition and CGS could explain cultural and genetic adaptations for parochial cooperation. While it is unclear exactly what types of adaptations can be hypothesized to exist via culture-led gene-culture coevolution, research on the proximate mechanisms underlying group cooperation does not seem to contain the footprint of intergroup competition as a strong selection pressure shaping group cooperation. Social psychology has discovered that parochial altruism can be motivated by both in-group love and out-group hate (Brewer 1999; Greenwald \& Pettigrew 2014). In-group love refers to the tendency to sacrifice immediate self-interest to benefit the in-group and its members; out-group hate refers to the tendency to sacrifice immediate self-interest to harm, derogate, and aggress competing out-groups and their members (Allport 1954; Brewer 1999; De Dreu et al. 2014). In-group love and out-group hate are conceptually and empirically distinct - in intergroup competition, humans may be motivated by in-group love, by out-group hate, or by some combination of both (De Dreu et al. 2014). Absent intergroup competition, or even the presence of other groups, humans may still be motivated by in-group love but, obviously, not by out-group hate. Intergroup competition is but one of the many drivers of in-group love.

Moreover, a meta-analysis of studies using cooperation games in psychology, economics, and sociology revealed robust intergroup discrimination in cooperation being due to in-group love rather than out-group hate (Balliet et al. 2014). Rivaling outgroups were treated the same way as unfamiliar strangers, suggesting that for cooperation it matters whether someone belongs to one's own group or not. Indeed, intergroup competition strengthens in-group love rather than out-group hate (Halevy et al. 2008; 2012), both among adults (De Dreu et al. 2014; Halevy et al. 2008) and young children (Buttelmann \& Böhm 2014). In fact, oxytocin, an evolutionary ancient neuropeptide pivotal in social bonding, modulates in-group love rather than out-group hate (De Dreu et al. 2010; 2011; Shalvi \& De Dreu 2014). Thus, in-group love can emerge both in the presence and absence of intergroup competition, and intergroup competition motivates in-group love more than out-group hate. Accordingly, there is substantial room for negotiated settlements and peaceful coexistence of (culturally) different groups and societies, especially when between group interactions are multi-dimensional and variable-sum.

Third and finally, intergroup competition may not be necessary to explain the (genetic) evolution of psychological adaptations supporting (parochial) cooperation. For example, group membership can be a cue that another person is part of an individual's social network and people may cooperate with in-group members to increase their reputation to gain indirect benefits from others and avoid being excluded from the group. Intergroup competition, or even intergroup comparisons, are not needed to bring about these processes (see Balliet et al. 2014; Yamagishi et al. 1999). Accordingly, a more parsimonious and empirically more valid perspective may be that cultural group selection is the outcome of individuals and their groups seeking to survive and prosper, and who, therefore, create norms and cultural practices that sustain and promote biologically prepared tendencies for in-group love. Some groups properly adapt to a changing environment (the entrance of competing out-groups included), and others are less able to adapt to changes in the environment (crop failures and earthquakes included). Whether exogenous pressures are social or non-social in nature, groups that organize and sustain ingroup love survive, prosper, and spread, and with them their culture. In short, in-group love, not intergroup competition, is driving cultural group selection.

\section{Human evolutionary history and contemporary evolutionary theory provide insight when assessing cultural group selection}

doi:10.1017/S0140525X15000102, e37

\section{Agustin Fuentes and Marc Kissel \\ Department of Anthropology, University of Notre Dame, Notre Dame, IN 46556. afuentes@nd.edu mkissel@nd.edu http://anthropology.nd.edu/faculty-and-staff/faculty-by-alpha/agustin- fuentes/ http://anthropology.nd.edu/faculty-and-staff/faculty-by-alpha/marc- kissel/}

Abstract: Richerson et al. provide a much needed roadmap for assessing cultural group selection (CGS) theory and for applying it to understanding variation between contemporary human groups. However, the current proposal lacks connection to relevant evidence from the human evolutionary record and requires a better integration with contemporary evolutionary theory. The article also misapplies the $F_{s t}$ statistic.

We agree that cultural group selection (CGS) has been under-theorized and applaud the attempt to develop a more formalized framework. However, there are two areas where the current effort falls short. The first is the lack of engagement with research on the development of complex cooperation and shared intentionality in our evolutionary history. The second area is twofold and involves the lack of explicit connection to many processes in the Extended Evolutionary Synthesis (EES; e.g., Laland et al. 2014; 2015), most of which can assist in developing robust CGS arguments. Richerson et al. also measure and describe cultural variation and culture groups, the key elements in CGS, using radically different, and often non-compatible, scales and definitions, relying on a weak analogy use of the $F_{s t}$ statistic. These flaws undermine the strength of the argument, but are not lethal and can be corrected.

Richerson et al. seek support for their assertions with data from contemporary psychology, ecology, economics, and cultural anthropology, but do so without providing engagement with the evidence for when or how human cooperation began, data that exist in the archaeological and fossil record. The drive toward complex cooperation and the incipient patterns of CGS are significantly older than suggested in the target article. There is evidence of complexity in cooperative behaviors deep in Hominoid (ape) history and complex cooperation between non-kin individuals is part of an adaptive complex that predates the appearance of the genus Homo (Herrmann et al. 2007; MacKinnon \& Fuentes 2011; Malone et al. 2012). In order to most effectively contextualize the emergence and role of CGS in the genus Homo, we must examine the Pleistocene evolutionary and archaeological record, which provides material evidence that can support these claims. The patterns and processes invoked in explaining CGS require that we have a working knowledge of the material, behavior, and ecological features of the expansive human niche across the Pleistocene. Recent work (Andersson et al. 2014; Fuentes 2015; Grove \& Coward 2008; Sterelny \& Hiscock 2014; Stout 2012; Whiten \& Erdal 2012) demonstrates that symbol-making and socio-cognitive niche construction played a key role in our lineage's success in the terminal Pleistocene and is directly relevant to understanding the evolutionary landscape of interactions within and between human groups. This lack of engagement (there are only a few paragraphs with scant citations) represents a missed opportunity to demonstrate the evolutionary history and processes central in developing the capacity for, and effects of, CGS across the evolution of the genus Homo. Combining the material record, niche 
construction theory, and paleoanthropological data sets creates a key venue to test the theories presented in the article.

For example, there is archaeological evidence for symbol construction, formation, and use that predates the appearance of Homo sapiens (circa 200,000 years ago). Initial results from our broad-scale assessment of these data indicate support for Richerson et al.'s assertion that CGS was active "as far back as symbolic marking is evident." While they suggest symbol-making may go back to 164 k.y.a., there are earlier signs such as the engraved bones from Bilzingsleben (Mania \& Mania 1988) which may date to 350 k.y.a. Ochre use older than 200 k.y.a has been uncovered at sites in Zambia (Clark \& Brown 2001), Sudan (Peer et al. 2004), and the Netherlands (Roebroeks et al. 2012), suggesting complex behavior and possible CGS at earlier dates. We suggest that key aspects of CGS emerged previous to identifiable symbol-making and then coevolved, via niche construction and selection (alongside drift and gene flow), with that capacity to develop a baseline for the radical leaps in complexity in the last 20,000 years that Richerson et al. focus on.

While we are supportive of the CGS concept, there is a lack of explicit connectivity to the Extended Evolutionary Synthesis (EES). Despite introducing three forms of cultural group selection, Richerson and colleagues retain a reliance on selectionbased processes as the key architect of function. This misses the opportunity to engage with niche construction, evo-devo, phenotypic plasticity, and other processes in the EES under which "Organisms are constructed in development, not simply 'programmed' to develop by genes. Living things do not evolve to fit into pre-existing environments, but co-construct and coevolve with their environments, in the process changing the structure of ecosystems" (Laland et al. 2014, p. 162). The processes in the EES, in addition to selection models, and multiple patterns of evolutionarily relevant inheritance (genetic, epigenetic, behavioral, and symbolic; e.g., Jablonka \& Lamb 2005) are central to the development of a more robust CGS approach. The current article does invoke non-genetic inheritance and implicitly uses a form of niche construction but does so without serious connection to the theoretical toolkit available. CGS will be more robust if it were explicitly connected to the EES.

We would be remiss if we did not note that cultural $F_{\mathrm{ST}}$ does not function in the same way as a genetic $F_{\mathrm{ST}}$ does. $F_{\mathrm{ST}}$ measures the variation that exists when a metapopulation lives in discrete groups. However, the assumption that responses to one or two questions on a survey are analogous to "one or two loci for a genetic $F_{\mathrm{ST}}$ " is not correct. Variance used to calculate $F_{\mathrm{ST}}$ in genetic or morphological contexts are based on physically definable measurements, but the answers by Saudi and Lebanese citizens to one of two questions about the role of clergy in the political system are not truly quantitative measures. They are influenced by multiple contextual and contingent (and linguistic) cultural variables and cannot be treated as independent material measures analogous to the number of alleles at a locus or inter-parietal breadth on a cranium. It is not clear that a cultural $F_{\mathrm{ST}}$ measures what a genetic one does or that such a measure is actually useful for assessing CGS.

CGS is relevant and should be incorporated into our understanding of human evolution, but approaches and analyses need to be rooted in a contemporary evolutionary theory and the archaeological record, be untangled from certain assumptions about selection and fitness, and seek models and assessments appropriate for cultural processes.

\section{Societal threat as a moderator of cultural group selection}

doi:10.1017/S0140525X15000114, e38

Michele J. Gelfand, ${ }^{a}$ Patrick Roos, ${ }^{b}$ Dana Nau, ${ }^{c}$ Jesse
Harrington, ${ }^{a}$ Yan Mu, ${ }^{a}$ and Joshua Jackson ${ }^{a}$
aDepartment of Psychology, University of Maryland, College Park, MD 20742; ${ }^{\mathrm{b}}$ Miner \& Kasch, 334 Scherer Lane, Severna Park, MD 21146; ' ${ }^{\circ}$ Department of Computer Science and Institute for Systems Research, University of Maryland, College Park, MD 20742.

mgelfand@umd.edu

www.gelfand.umd.edu

patroos@gmail.com

nau@cs.umd.edu

http://www.cs.umd.edu/users/nau

jesserh@umd.edu

https://sites.google.com/site/jharringtonumd/

yanmu@umd.edu

jjackso1@umd.edu

Abstract: As scholars have rushed to either prove or refute cultural group selection (CGS), the debate lacks sufficient consideration of CGS's potential moderators. We argue that pressures for CGS are particularly strong when groups face ecological and human-made threat. Field, experimental, computational, and genetic evidence are presented to substantiate this claim.

The debate on cultural group selection (CGS) would be more fruitful if it considered the role of human ecology as a critical moderator of CGS. In particular, we argue that ecological and historical threats to human societies strengthen CGS pressures. As we summarize below, there is substantial evidence from field, experimental, computational, and genetic studies suggesting that exposure to threat increases the strength of social norms and punishment of deviance, which are conditions that Richerson et al. identify as important mechanisms that maintain intergroup variation. Hence, ecological and historical threats can critically moderate the strength of CGS pressures and may provide insight into precisely where, when, and to what extent CGS occurs.

Field research across 33 nations first documented the connection between ecological and historical threat and the degree of norm strength in groups (Gelfand et al. 2011). Ecological and human-made threats were theorized to increase the need for clear rules and coordination for the purpose of survival - whether it is to reduce chaos in nations that have high population density, to effectively deal with resource scarcity, to coordinate in the face of natural disasters, to defend against territorial threats, and/ or to contain the spread of infectious disease. Societies facing these ecological and human challenges therefore develop strong norms and sanctions for deviance (i.e., are tight) to enhance coordination and deal effectively with such threats. By contrast, societies with less threat have a lower need for coordination, and hence are loose, that is, have weaker norms and much higher tolerance for deviant behavior. Using archival and survey data, we tested this hypothesis across a wide range of variables and found that societies facing ecological and historical threats do indeed develop stronger norms and punishment of deviance (Gelfand et al. 2011).

Further research confirmed this relationship at the state level in the United States. Harrington and Gelfand (2014) found that tighter states (e.g., those which have strong norms and punishment of deviance) have a greater degree of natural disasters and environmental vulnerabilities, fewer resources (indicated by greater rates of food insecurity), a greater incidence of disease and health vulnerabilities, and perceive a greater degree of external threat, reflected in the desire for more national defense spending and greater rates of military recruitment, as compared to loose states. (i.e., those which have weaker norms and less punishment of deviance). Individuals in tighter states also had higher levels of conscientiousness - a personality dimension that reflects greater impulse control, cautiousness, desire for orderliness, and conformity to norms - and lower openness - a personality dimension that reflects non-traditional values and beliefs, interest and curiosity toward new ideas, and tolerance for other cultures (John et al. 2008). Tighter states also had significantly lower residential mobility and supported more isolationist policies (such as buying American products exclusively and supporting government restriction of imported products) compared to loose states. 
Beyond these correlational results, evolutionary game theoretical (EGT) models show that groups that face a high degree of threat require stronger norms and greater punishment of deviance in order to survive (Roos et al. 2015). Following existing EGT models (Hilbe \& Traulsen 2012; Roos et al. 2014), we examined the evolution of strategies for cooperation as well as strategies for a subsequent punishment phase in cooperation and coordination games. We found that exposing populations to higher degrees of threat-implemented through lower base-rate payoffs - led to an increase in the survival of agents that adhered to the cooperation or coordination norm and those that punished norm-deviating behaviors. Under low threat, the evolutionary pressures resulted in a more diverse mix of all possible strategies. Besides showing that strong norms and punishment of deviance emerges under chronic levels of high threat, these models also found that temporary increases in threat cause norm strength to increase until the threat subsides. Given that conformity and punishment are important pressures for CGS (per Richerson et al.), it follows that increases in ecological and historical threat contribute to the conditions for CGS to occur.

Our electroencephalography work has also found neurobiological differences in the detection of deviant behaviors among tight and loose groups (Mu et al. 2015). Chinese samples, which reported higher degrees of territorial threats, had stronger neurological responses (e.g., greater $\mathrm{N} 400$ ) in the frontal region when viewing social norms violations (e.g., Bob is in the library, he is shouting as compared to Bob is in the library he is studying) in comparison with U.S. samples. Moreover, stronger N400 responses mediated cultural differences in a number of attitudes and behaviors, including higher self-control and ethnocentrism, but lower creativity, among Chinese as compared to U.S. groups. More generally, pronounced neural reactions to norm violations should enable groups to enforce social norms and punish deviant behavior, which, according Richerson et al., should further enhance intragroup cohesion and intergroup variation.

Relatedly, using genetic data, Mrazek et al. (2013) argue that higher frequencies of short (S) allele in the 5-HTTLPR polymorphism of the serotonin transporter gene (SLC6A4) persist in regions of the world prone to ecological threat because heightened threat sensitivity may be adaptive in such regions. Because the $\mathrm{S}$ allele has been associated with more aversive reactions to moral violations in groups, they suggest that threat-driven genetic variance in the $\mathrm{S}$ allele may underlie differences in moral attitudes across cultures. Their path model linking ecological threat, genetic variability of the $\mathrm{S}$ allele, the strength of social norms, and reactions to moral violations provides plausible evidence for this argument.

In sum, as scholars have rushed to either prove or refute cultural group selection, few have explored it as a phenomenon that is modified by the ecological and historical contexts in which human groups are embedded. Considering the role of societal threat in theories of CGS could help resolve the debate of where, to what extent, and how CGS occurs.

\section{A framework for modeling human evolution}

doi:10.1017/S0140525X15000126, e39

\section{Herbert Gintis \\ Santa Fe Institute, 1399 Hyde Park Road, Santa Fe, NM 87501. hgintis@comcast.net \\ http://people.umass.edu/gintis}

Abstract: Culture-led gene-culture coevolution is a framework within which substantive explanations of human evolution must be located. It is not itself an explanation. Explanations depend on such concrete historical evolutionary factors such as the control of fire, collective child- rearing, lethal weapon technology, altruistic cooperation and punishment, and the mastery of complex collaboration protocols leading to an effective division of social labor.

The target article nicely elaborates the strong relationship between cultural group selection and gene-culture coevolution. Richerson et al. correctly observe that gene-culture coevolution significantly strengthens genetic group selection models. However, there really are no purely genetic group selection models in the literature. All such models employ the phenotypic gambit (Grafen 1984), in which complex transmission processes are treated as though they were the product of a single allele of the genome. Such models represent equally any transmission process, cultural, genetic, or interaction between the two, that depend only on vertical transmission from parents to offspring (Gintis 2014).

The target article's contention that cultural group selection is "a basic explanation for our species' highly unusual ability to create large societies with widespread cooperation between non-relatives" (sect. 7, para. 2) is incorrect. Cultural group selection is not an explanation of anything. Rather, it is a framework within which such an explanation can fruitfully be developed. The main features of human cooperation, which include collective child-rearing without a reproductive division of labor (Hrdy 2000; Wilson 2012), hunting large game with lethal weapons (Wrangham \& Carmody 2010), altruistic cooperation and punishment (Bowles \& Gintis 2011), as well as collaborative skills depending on a theory of mind (Tomasello 2008), follow from the particular evolutionary history of our species.

Curiously, the authors suggest that there is evidence for culture-led gene-culture coevolution only "for a few simple genetic traits" (sect. 2.2, para. 6). In fact, there is overwhelming evidence for this process as central to the constitution of Homo sapiens, a few pieces of which I will cite here.

Human Self-domestication: Darwin noticed that selective breeding of mammals for tameness entailed a pattern of similar side-effects of domestication to human society in distinct species. Darwin even suggested that, "Man in many respects may be compared with those animals which have been long domesticated" (Darwin 1871, Ch. 7, p. 172). Belyaev (1979) corroborated this insight, studying captive silver foxes bred for tameness. These animals developed humanly attractive faces with short snouts, floppy ears, patches of white fur on their heads, and curly tails (Gibbons 2014). More recently, Cieri et al. (2014) documented domesticated syndrome changes in human evolution since the Middle Stone Age and Upper Paleolithic, and Wilkins et al. (2014) have proposed a general genetic model explaining the domestication phenomenon.

This is evidence for a very straightforward culture-led group selection mechanism in which an increasingly complex division of labor and social norms that rewarded cooperation (Tomasello 2014) favored genetic changes that produced a more domesticated and prosocial human disposition.

Control of Fire and the Reorganization of the Human Upper Torso: Prior to the control of fire, hominins inhabited trees at night as a defense against predators. Because predators have an instinctive fear of fire, the control of fire permitted hominins, who were already bipedal, to abandon climbing almost completely (Wrangham \& Carmody 2010). The lack of need for brachiation freed the hand, arms, and shoulders of proto-humans to evolve for other purposes (Gintis et al. 2015).

Lethal Weapons and the Physiology of Throwing: Hominins developed the use of long-range projectile weaponry, and such techniques were central to human social life (Bingham 1999; Wilkins et al. 2012). Humans are unique in possessing the neural machinery for rapid manual-brachial movements that allows for precision stone-throwing, which depends on the brain's capacity to orchestrate a series of rapidly changing muscle movements (Calvin 1983). 
Roach et al. (2013) showed that Homo erectus had already evolved this capacity for accurate overhead throwing. Humans are unique in possessing a torso musculature optimized for the powerful and accurate throwing of projectile weapons.

Language and the Physiology of Communication: The increased social importance of communication in human society rewarded genetic changes that facilitate speech. Regions in the motor cortex expanded in early humans to facilitate speech production. Concurrently, nerves and muscles to the mouth, larynx, and tongue became more numerous to handle the complexities of speech (Jurmain et al. 1997). Parts of the cerebral cortex, Broca's and Wernicke's areas, which do not exist or are relatively small in other primates, are large in humans and permit grammatical speech and comprehension (Belin et al. 2000; Binder et al. 1997).

Modern humans have a larynx low in the throat, a position that allows the throat to serve as a resonating chamber capable of a great number of sounds (Relethford 2007). The first hominids that had skeletal structures supporting this laryngeal placement were the Homo heidelbergensis, who lived from 800,000 to 100,000 years ago. In addition, the production of consonants requires a short oral cavity, whereas our nearest primate relatives have much too long an oral cavity for this purpose. The position of the hyoid bone, which is a point of attachment for a tongue muscle, developed in Homo sapiens in a manner permitting highly precise and flexible tongue movements.

\section{How evolved psychological mechanisms empower cultural group selection}

doi:10.1017/S0140525X15000138, e40

\author{
Joseph Henrich ${ }^{\mathrm{a}, \mathrm{b}}$ and Robert Boyd ${ }^{\mathrm{c}}$ \\ ${ }^{a}$ Department of Human Evolutionary Biology, Harvard University, Cambridge, \\ MA 02138; ${ }^{\mathrm{b}}$ Department of Psychology, Department of Economics, University \\ of British Columbia, Vancouver, BC V6T 1Z4, Canada; ' $S$ chool of Human \\ Evolution and Social Change, Arizona State University, Tempe, AZ 85287. \\ joseph.henrich@gmail.com robert.t.boyd@gmail.com \\ http://www2.psych.ubc.ca/ henrich/ \\ http://robboyd.abcs.asu.edu/
} practices can evolve in ways that more effectively tap into a wide variety of evolved psychological mechanisms to foster group-beneficial behavior. The more powerful such evolved mechanisms are, the more effectively culture can potentially harness and manipulate them to generate greater phenotypic variation across groups, thereby fueling cultural group selection.

Many critics of the approach taken by Richerson et. al. incorrectly believe that evolutionary mechanisms regulating reciprocity, reputation, life history, and behavior toward kin necessarily represent alternatives to cultural group selection as explanations for cooperative behavior, and that evidence for these mechanisms constitutes evidence against a role for cultural group selection (e.g., Lamba \& Mace 2011; Pinker 2012). To the contrary, intergroup competition will favor those group-beneficial cultural traits - including social norms, beliefs, and practices - that most effectively infiltrate and exploit aspects of our evolved psychology (N. Henrich \& Henrich 2007; Richerson \& Boyd 1999). Rituals, religious beliefs, marriage norms, and kinship systems all tap into how the mind works in different ways, and if these traits vary in ways that influence the success of groups in competition, then cultural group selection can shape human social behavior. The following examples illustrate this point.

The kinship systems that dominate life in small-scale societies variously harness, extend, and suppress evolved psychological mechanisms for dealing with relatives (Mathew et al. 2013). By building on the intuitions and motivations supplied by our evolved kin psychology, cultural evolution can, for example, spread social norms for treating distant cousins more like genetic siblings, thereby fostering greater cooperation while inhibiting sex and marriage. Such kinship norms often exploit our proximate kin identification mechanisms by influencing patterns of residence and daily routines (e.g., who eats together), and labeling (e.g., calling some cousins "brothers"). Incest taboos can activate a proximate mechanism for innate incest aversion by prescribing the co-rearing of cousins in the same extended household. Moreover, third parties readily acquire such norms because they already have compatible intuitions about how others "should" behave toward their siblings (J. Henrich 2016).

Widespread unilineal clan organizations are particularly interesting. Though clearly rooted in kin psychology, they devalue half of one's genealogical relatives in order to foster greater cooperation with the other half. Among foragers in Indonesia, field studies show that patriclan membership predicts large-scale cooperation in whale hunting better than genealogical kinship (Alvard 2011). In Australian foragers, ethno-historical and linguistic reconstructions suggest that patrilineal clan organizations spread only in the last 6,000 years, probably via various forms of intergroup competition (Evans \& McConvell 1998; J. Henrich 2016).

Cultural evolution may also empower direct reciprocity (N. Henrich \& Henrich 2007). Theoretical work (Boyd \& Lorderbaum 1987) reveals that the success of reciprocating strategies depends on the particular constellation of other strategies present (e.g., see Zefferman [2014a] on Delton et al. [2011]). The combinatorial explosion of possibilities in this complex multi-dimensional space of possible strategies means that it is unlikely that a jukebox-like psychology could effectively address this challenge. Thus, it is not surprising that outside of humans reciprocity is rare and limited to low cost behaviors (Clutton-Brock 2009).

Cultural evolution, however, may explain why reciprocity is so powerful in humans (Boyd \& Mathew 2015; N. Henrich \& Henrich 2007). Social norms provide shared standards of acceptable behavior, allowing third parties to assist in identifying and punishing defectors. Intergroup competition can favor those social norms which maximize the effectiveness of direct reciprocity under particular conditions, and this may help explain why the importance of direct reciprocity varies so dramatically among societies (Fiske 1992). Thus, it may be cultural evolution that turns direct reciprocity from the flimsy and relatively unimportant meta-strategy that we see in other animals into a powerful force for cooperation.

Reputation underpins many models of cooperation (Barclay 2013; Panchanathan \& Boyd 2004). However, such models are incomplete because they leave unspecified where the required reputational standards come from. Reputational standards are culturally transmitted (Salali et al. 2015) and vary dramatically among societies (Bell et al. 2009), even among societies facing similar ecological circumstances (Edgerton 1971; McElreath 2004). Across societies, reputations are influenced not only by cooperative actions like contributing to village feasts or leading the charge against the village in the next valley, but also practices like female infibulation, funerary cannibalism, ritual participation, and food taboos. Thus, any explanation that "reputation explains cooperation" needs a theory for why reputational standards vary so dramatically among societies, and why group-beneficial behaviors often generate good reputations. Driven by intergroup competition, cultural evolution may favor some elements of reputational content (e.g., for bravery in warfare) over other elements.

Finally, environmental cues may evoke evolved psychological responses that influence human sociality. For example, some argue that cues received early in life evoke either a "fast" or "slow" life history strategy (McCullough et al. 2013), with cues of safety, security, and stability favoring "slow" life histories, and greater cooperativeness. Building on this, some argue, often in opposition to cultural evolutionary accounts, that such life history switches account for between-group variation in 
prosociality and the growth of moralizing religions (Baumard et al. 2015).

However, what's missed is that such evoked responses are precisely the kind of psychological switches that cultural group selection could harness. If slow life history strategies favor greater cooperation, then cultural group selection will favor sets of norms that stabilize families, provide social safety nets, reduce disease threats, or do whatever most effectively throws the switch in ways that foster success in intergroup competition. The existence of such switches can actually increase the variation among groups in phenotypes, fueling the engine of cultural group selection. The spread of normative monogamous marriage provides an example of an institution that harnesses various evolved mechanisms to increase paternal investment, household relatedness, and infant/child survival while reducing male-male competition (Henrich et al. 2012b). This culturally evolved package is precisely the kind of institution that could throw the slow life history "switch" and magnify the power of cultural group selection.

Overall, the existing evidence for the importance of kinship, reciprocity, reputation, and evoked responses for human cooperation and sociality contributes to a prima facie case for cultural group selection by providing psychological mechanisms that can be exploited by relatively weak social norms to generate big differences in phenotypes between groups, thereby powering up cultural group selection. We urge researchers to consider a more integrative approach, one that synthesizes genetic and cultural evolution.

\section{Does cultural group selection explain the evolution of pet-keeping?}

\author{
doi:10.1017/S0140525X1500014X, e41
}

\section{Harold Herzog}

Department of Psychology, Western Carolina University, Cullowhee, NC 28723.

herzog@email.wcu.edu

http://paws.wcu.edu/herzog/

Abstract: Pet-keeping is highly variable across cultures in both frequency and form. Cultural group selection offers a plausible explanation for the development and spread of this uniquely human phenomenon in that pet-keeping involves an inheritance system, socially transmitted norms and preferences, substantial between-group variation, and (albeit indirectly) intergroup competition.

In the target article, Richerson et al. argue that cultural group selection (CGS) was essential for the evolution of the high level of cooperation seen in our species, as well as the development of institutions such as religions and businesses. Here I make the case that CGS also provides a coherent explanation for the evolution of a uniquely human form of social relationships-pet-keeping. Pet-keeping poses an evolutionary problem. There is no evidence that pets increase the reproductive potential of their owners, and the putative health and psychological benefits of living with animals are not as well-established as commonly believed (Herzog 2011). Maintaining a companion animal can be costly, and devoting affection and resources on members of another species that have no apparent function cannot be explained by kin selection or reciprocity.

Several lines of evidence suggest that CGS may have played a role in the emergence and spread of pet-keeping. First, petkeeping occurs in humans only. While nonhumans occasionally form attachments to members of a different species, these unusual relationships always seem to involve some human intervention such as occurs in zoos, wildlife parks, and households (Herzog 2014). In completely natural environments, "animal odd couples" analogous to pet-keeping are extremely rare and probably non-existent. Nonhuman species do possess prosocial motivational systems such as parental care and empathy that are prerequisites for the formation of interspecific attachments (e.g., Preston \& De Waal 2002). But the fact that long-term affectionate relationships between non-conspecifics have not been observed in the wild, even in nonhuman apes, suggests that petkeeping requires the degree of rapid social learning found only in our species.

Second, unlike language, music, and prohibitions against sex with first-degree relatives, pet-keeping is not a human universal. Indeed, the frequency and form of pet-keeping vary widely among cultures. Indigenous peoples of Amazonia, for example, commonly make pets of captured wild animals such as monkeys and birds. This practice, however, is rare among tribal peoples of New Guinea (Diamond 1993). In some cultures, pet-keeping is unknown, and some languages have no word that corresponds to "pet." Cross-cultural studies reveal that practices common in modern Western societies, such as letting animals live in our homes, allowing them to sleep in our beds, and considering them full-fledged family members are cultural anomalies (Gray \& Young 2010). Further, social norms governing relationships with pets can shift dramatically over time. In Medieval Europe, cats were routinely subjected to torture and mass slaughter, as they were linked with witchcraft. Their image only began to change in the mid-17th century, with the complete transformation of the cat from pariah to household pet occurring by the end of the 18th century (Bradshaw 2013).

Third, preferences for pets are transmitted vertically from parent to child and horizontally across cultural landscapes. A recent study found that mothers who had pets in their childhood, tended to raise their offspring with pets (Westgarth et al. 2010). Sudden shifts in the popularity of types of pets occur across cultural groups via social transmission. In the United States, examples include transient enthusiasms for baby turtles, caged songbirds, ferrets, mice, and pot-bellied pigs. Using 60 million American Kennel Club dog registrations between 1926 and 2005 as data, my colleagues and I have shown that shifts in choices for canine companions are influenced by the mechanisms Richerson et al. propose as sources of intergroup variation. These include rapid social learning, conformity, and the influence of prestigious models. We have found that dog breed preferences are influenced by the decisions of others (Herzog et al. 2004); are unrelated to breed-characteristic qualities such as desirable behavioral traits and frequency of genetic diseases (Ghirlanda et al. 2013); and result in fads in which the more quickly a breed becomes popular, the faster its appeal subsequently declines (Acerbi et al. 2012; Herzog 2006). In addition, long-lasting changes in the popularity of types of dogs are sometimes instigated by media exposure (films with canine movie stars) (Ghirlanda et al. 2014). In short, pet-keeping follows the laws of fashion.

Fourth, while it is unlikely that cultural differences in petkeeping are the direct result of intergroup competition, competitive forces do shape aspects of human-animal interactions that are precursors to affection. The use of hunting dogs varies widely among indigenous peoples of the Neotropics. Dogs substantially increase the success of Mayangna hunters in Nicaragua, and while the Mayangna are not attached to adult dogs, they do name their dogs and play with puppies (Koster 2009). The acquisition of horses by Apache and Comanche Indians in the 17th century profoundly changed the nature of tribal warfare on the Great Plains of the United States (Hämäläinen 2003).

Finally, pet-keeping practices are affected by some of the institutions that Richerson et al. believe arose via CGS, for example, religion and businesses. On the multi-ethnic island of Sri Lanka, $79 \%$ of Buddhist households include a dog, compared to Muslim households, of which only $4 \%$ do (Knobel 2009). In the United States, over the last two decades, the proportion of homes with companion animals has increased only slightly while the amount of money Americans spend on their pets has risen 
Commentary/Richerson et al.: Cultural group selection plays an essential role in explaining human cooperation

fivefold. It is likely that efforts by industry trade groups such as the Pet Products Manufacturers Association and the American Veterinary Medical Association to promote the benefits of pet ownership have contributed to the recent cultural trend sometimes referred to as the "humanization of pets."

In sum, pet-keeping shows the marks of a cultural group-selected institution. It involves an inheritance system, socially transmitted norms, a high degree of between-group variation, and (albeit indirect) intergroup competition. Certainly, pet-keeping has evolutionary and neurobiological roots (see, e.g., Archer 1997; Stoeckel et al. 2014). But the large individual and cultural differences observed in attachment to animals suggest that learning and culture play a larger role than biology when it comes to bringing them into our lives. CGS offers an elegant explanation for an important cultural phenomenon that has, until recently, been neglected by social and behavioral scientists.

\section{Frozen cultural plasticity}

\section{doi:10.1017/S0140525X15000151, e42}

\section{Petr Houdek ${ }^{\mathrm{a}, \mathrm{b}, \mathrm{c}}$ and Julie Novakova ${ }^{\mathrm{b}}$ \\ ${ }^{\mathrm{a}}$ Faculty of Social and Economic Studies, J. E. Purkyne University in Usti nad Labem, CZ-40096 Usti nad Labem, Czech Republic; ' Department of Philosophy and History of Science, Faculty of Science, Charles University in Prague, CZ-12808 Prague 2, Czech Republic; ' Faculty of Business Administration, University of Economics, Prague, CZ-130 67 Prague 3, Czech Republic. \\ petr.houdek@gmail.com julie.novakova@gmail.com http://web.natur.cuni.cz/ houdek3/}

Abstract: We discuss cultural group selection under the view of the frozen plasticity theory and the different explanatory power and predictions of this framework. We present evidence that cultural adaptations and their influence on the degree of cooperation may be more complex than presented by Richerson et al., and conclude with the gene-environmentculture relationship and its impacts on cultural group selection.

Richerson et al. present compelling evidence for cultural group selection acting as a major constituent of human cooperation; however, we argue that it would be more beneficial to view cultural evolution in light of more complex evolutionary models, such as the punctuated equilibrium theory or the frozen plasticity theory (Flegr 2010): frequency-dependent selection of cultural norms and, mostly, punctuated equilibrium pattern of cultural evolution (in contrast to the simple gradualist approach in Richerson et al.'s target article).

The frozen plasticity framework takes into account the relations between the cultural norms, which are seldom unrelated to each other (a cultural trait can be a positive norm in one context, but a harmful one in another context). The result could be that the more institutions a culture develops, the more "frozen" it becomes as the traits interact, and it becomes increasingly difficult for any new (better) variant to become established, especially if the culture is isolated.

An example of our point consists in thriving Neolithic agricultural societies, which formed large and successful autocratic cultures, but in which high power inequality and rent-seeking emerged. These factors eventually became barriers to their further development and prevented efficient cooperation, as the magnitude of relationships between various institutions grew so large that any change would require too large a restructuralization of the status quo (Olsson \& Paik 2013). Major institutional changes are likely only after the occurrence of large-scale events or disasters - natural as well as socioeconomic. (An example of this is that, in the aftermath of World War II, Japan ended state Shintoism and hundreds of religious groups sprang up in a very quick succession; Iannaccone 1998.) But there is no place for such developments in Richerson et al.'s framework; in their view, societies implement cooperative norms and irreversibly move toward prosperity.

We would like to stress that adjustment toward better-performing norms ("cultural plasticity") is more intricate than Richerson et al. suggest. We show this in the following sections.

The unbearable lightness of good institutions. Culture adoption elasticity is highly dependent on the geographical, historical, and intragroup factors and on development of competing cultures, but the direction of influence is not straightforward (Acemoglu et al. 2005). For example, Europeans were more likely to introduce extractive colonial regimes in relatively richer societies or regions where they could not settle. These regions struggle with poverty now. On the contrary, in relatively poor regions Europeans settled in large numbers and implemented institutions that encourage prosperity today (Acemoglu et al. 2001; 2002). Richerson et al. would view the story in terms of pure natural selection, but their general theory does not distinguish among factors such as culture and institutions versus human capital (Chanda et al. 2014; Glaeser et al. 2004) versus genetic diversity (Ashraf \& Galor 2013; Cook 2014), which co-influence intergroup competition.

Moreover, similar types of culture originating from one ancestral culture may choose similar environments to colonize, thereby creating a logically consistent phylogenetic tree while not excluding the evoked culture hypothesis. The character of natural environment may have influenced the relative ability of different cultures to expand and prevail over others; thus institutional variation could be only a derivative force in intergroup competition (Spolaore \& Wacziarg 2013).

(Mal)adaptivity of institutions and norms in cooperation. Richerson et al. neglect to stress that some norms can be beneficial on a certain level and harmful on another by moving a culture to the non-cooperative equilibrium. They mention the benefits of institutionalized norms like honor and bravery to intergroup warfare; however, these can act harmfully at the intragroup level. The culture of honor within high castes in India is related to a low degree of coordination (Brooks et al. 2014).

Norms that had become "frozen" may have a detrimental effect in many modern societies, expressed, for instance, in the higher prevalence of antisocial punishment (Kuběna et al. 2014) in countries whose strong emphasis on traditions may have been beneficial previously (Gintis 2008; Herrmann et al. 2008). While some norms could have played an essential role in forming human cooperation, many likely ceased fulfilling this role due to the decreased adaptivity of the trait-network. Important examples of these phenomena could be religious norms (Norenzayan et al. 2016).

Selection forces acting on meta-norms. Richerson et al. establish the government as an intelligent designer. Their examples include patent law and antimonopoly law. However, these traits are more in accord with our argument that social norms become "frozen" in time. They persist largely due to powerful special interest groups, even if they become harmful for the society. Patent law in its current form impedes rather than promotes innovation (Boldrin \& Levine 2008), and antimonopoly law is often a tool of adjustment of government politics (Armentano 1986) or a product of lobby groups' pressure.

The meta-norms (i.e., norms promoting punishment of defectors and punishment-shirkers alike) are subject to selection as well. Prietula and Conway (2009) note that an established or internalized meta-norm could easily become a "trap" and stop being beneficial.

Gene-culture coevolution. Genetic diversity has greater effects on comparative development than Richerson et al. admit. Genetic make-up can influence aspects of cultural evolution, which along with resource accessibility may in turn give advantage to bearers of certain genetic markers in a gene-culture coevolutionary process, for example, in the interaction of scents and genetic make-up (Havlícek \& Roberts 2013). Loci related to olfaction, immunity, and metabolism (connected to subsistence strategies) are among those most positively selected in recent human history (Akey 2009).

Conclusion. We fully support cultural selection as the principal explanation for the degree of cooperation among humans (see a 


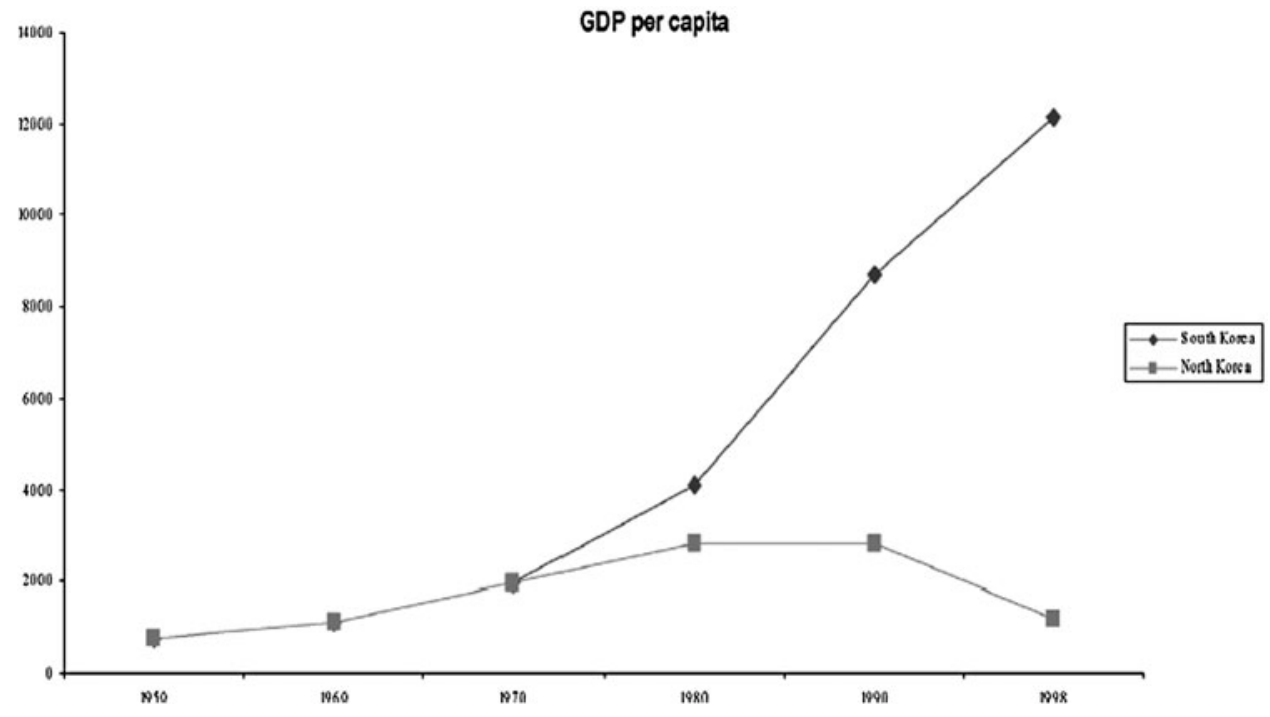

Figure 1 (Houdek \& Novakova). Gross domestic product (GDP) per capita in North and South Korea, 1950-98. Reprinted with permission from Acemoglu et al. (2005).

contemporary example in our Fig. 1: North and South Koreans shared the same history and cultural, linguistic, and geographic roots, but institutional differences dramatically diverged the development of their countries). Nevertheless, we suggest a rigorous analysis of the political and population structure of societies throughout their history to assess whether "cultural bottlenecks" and founder effects influence the plasticity of cultural traits and can explain human cooperation in greater detail.

\section{The sketch is blank: No evidence for an explanatory role for cultural group selection}

doi:10.1017/S0140525X15000163, e43

\section{Max M. Krasnow ${ }^{a}$ and Andrew W. Delton ${ }^{b}$ \\ a Department of Psychology, Harvard University, Cambridge, MA 02138; ${ }^{\mathrm{b}}$ Department of Political Science, College of Business, Stony Brook University, Stony Brook, NY 11794-4392. \\ krasnow@fas.harvard.edu andrew.delton@stonybrook.edu http://projects.iq.harvard.edu/epl/people/max-krasnow http://andrewdelton.com}

Abstract: As evidence that cultural group selection has occurred, Richerson et al. simply retrodict that humans use language, punish each other, and have religion. This is a meager empirical haul after 30 years. This contrasts sharply with the adaptationist approach to human behavior - evolutionary psychology - which has produced scores of novel, specific, and empirically confirmed predictions.

The adaptationist approach of George Williams (1966) revolutionized evolutionary biology. It replaced a loose willingness to assume that every trait of an organism had an evolved function with, instead, a rigorous focus on characterizing adaptations - traits of organisms shaped by natural selection to solve an adaptive problem. Not all traits are adaptations: some are merely incidental by-products of adaptations and need no special adaptive explanation. Blood contains adaptations for the transport of oxygen and carbon dioxide; its transport properties were crafted by natural selection. Its red color when oxygenated is a by-product of its chemistry and needs no adaptive explanation. In Williams' words, "adaptation is a special and onerous concept that should be used only where it is really necessary” (p. 4). Researchers must carefully test whether a trait is in fact an adaptation and not recognize adaptation in "purely fortuitous effects" or invoke natural selection "to resolve problems that do not exist" (p. 4). The adaptationist program has been enormously productive: By the 1990s, after 30 years of work, the fruit of this approach already filled textbooks (e.g., Alcock 1998; Krebs \& Davies 1993), and it is now the background assumption of virtually all research in animal behavior (Alcock 2001).

Williams had a further insight: Selection is most efficient at the lowest, most faithfully replicating levels in the hierarchy of life. Genes reproduce more faithfully than individuals, who reproduce more faithfully than groups. Thus, adaptions generally arise in the service of transmitting the genes that build them, not in the service of perpetuating the groups where individuals carrying those genes reside. But, as Williams noted, a herd of fleet deer is also a fleet herd. So, how was fleetness built? Parsimony says that, because genic selection is more powerful, unless herd fleetness shows special design that could not be preserved simply by the differential reproduction of speedier deer, group selection is not doing any explanatory work. Group selection should only be invoked with clear evidence of group-level adaptation, not merely group-level phenomena, an error repeatedly made by the target article.

Theories of cultural group selection, gene-culture coevolution, and dual inheritance are now at least 30 years old (e.g., Boyd \& Richerson 1985). Thus, we think it is fair to question their empirical yield. Have these theories made detailed, unique, and novel predictions confirmed with evidence showing specifically groupfunctional adaptation? Or has group-functional adaptation been applied when not necessary to solve problems that do not exist?

Our reading of the target article's section 6 suggests the latter. The best evidence offered for cultural group selection is the retrodiction that humans have language, sometimes punish each other, and have religion. There are no novel predictions made by cultural selection theory. (Simply re-describing known facts with the terminology of cultural group selection is not a novel prediction.) More critically, no evidence is offered that these traits arise from cultural group selection; we argue that apparent group-level effects are by-products arising from individual-level benefits when this alternative is taken seriously.

The evoked culture and cognitive niche (a.k.a. improvisational intelligence) hypotheses that Richerson et al. discard are two instances of this alternative. Evoked culture hypotheses propose facultative cognitive mechanisms that modulate behavior based on available information (Tooby \& Cosmides 1992). These 
mechanisms evolve when, ancestrally, different behaviors are best in different physical and social ecologies. This was surely the case for punishment, as the utility of using coercive power to get one's way would have ancestrally varied between people (e.g., aggression pays more for the strong; Sell et al. 2009) and between ecologies (e.g., aggression pays more when there are gains it can recruit; Krasnow et al. 2012). Cognitive niche hypotheses propose mechanisms that extract information from the environment and make appropriate inferences (Tooby \& DeVore 1987).

Importantly, these theories are not the straw-men reported in the target article. Why should a mechanism for exploiting the cognitive niche rely on solitary innovation when a clearly superior design would also target information in the minds of others? Why should a mechanism for evoked culture be unable to operate outside the parameter range under which it evolved? Our mechanisms of diet choice gravitate to big macs and cheesecake despite none existing during our evolutionary history. Although police forces and the rule of law did not exist in Paleolithic society, our evolved psychology of personal aggression can be exploited by these institutions to evoke cultures that never existed before. To the extent that our evolved social psychology is richly articulated, features of this evoked culture can act as further inputs to other facultative mechanisms and evoke further novel cultural features (Tooby \& Cosmides 1992).

A final note: We are surprised that a paper discussing the taxonomy of group selection and cultural coevolutionary models can so repeatedly mistake simple cultural change for cultural group selection. There is no dispute that cultures change over time. This is, in fact, the predicted result when creatures exploiting the cognitive niche leave enduring traces in their societies that evoke new cultures down the line (Pinker 2012). But as Williams argued, adaptation is an onerous concept. It is no less onerous for culturally group selected adaptations. The burden has not been met here.

In our view, the jury is still out on the explanatory and predictive power of cultural group selection theory. Luckily, it is not the only approach on offer. About 30 years ago another theoretical approach emerged as the confluence of adaptationism and cognitive science: evolutionary psychology (e.g., Daly \& Wilson 1988; Tooby \& Cosmides 1989). Although Richerson and colleagues criticize evolutionary psychological approaches, it is evolutionary psychology that has inspired scores of novel, specific, and empirically confirmed predictions. The many empirical successes of evolutionary psychology have filled textbooks, handbooks, and hundreds of journal articles (e.g., Buss 2005; 2014; Buss \& Reeve 2003). Although cultural group selection theory may yet yield insights, at the moment it is evolutionary psychology that provides a generative, testable, and productive framework for understanding the human mind and the cultures it creates.

\section{The empirical evidence that does not support cultural group selection models for the evolution of human cooperation}

doi:10.1017/S0140525X15000175, e44

\section{Shakti Lamba \\ Centre for Ecology and Conservation, College for Life and Environmental Sciences, University of Exeter, Penryn Campus, Penryn, Cornwall TR10 9FE, United Kingdom. \\ s.lamba@exeter.ac.uk \\ http://biosciences.exeter.ac.uk/staff/index.php?web_id=Shakti_Lamba}

Abstract: I outline key empirical evidence from my research and that of other scholars, testing the role of cultural group selection (CGS) in the evolution of human cooperation, which Richerson et al. failed to mention and which fails to support the CGS hypothesis.
Much of my research has focused on seriously testing the cultural group selection (CGS) hypothesis for the evolution of large-scale cooperation in humans as presented by Richerson et al. CGS is an elegant idea that captured my imagination, among those of many others. But a scientist must always remain open to the possibility that even the most elegant of ideas can fail to explain the real world. As Thomas Huxley said, "the great tragedy of Science [is] the slaying of a beautiful hypothesis by an ugly fact" (Huxley 1894, p. 244).

I agree with Richerson et al. that the issue is not whether cooperation based on reciprocity and inclusive fitness exists, but rather whether these explanations can account for large-scale cooperation in humans. I also agree that group selection on cultural variation is theoretically possible, but that the extent of variation, heritability, and competition will determine whether it is an empirically important force or not. Richerson et al. ask, "Are the norms that underpin institutions among the cultural traits so transmitted? Do we observe sufficient variation at the level of groups of considerable size for group selection to be a plausible process?" (target article, Abstract). They state that "If the answer to one of these questions is 'no,' then we must look to other hypotheses" (Abstract). My research, as well as that of others, suggests that the answer to both these questions may be "no."

A series of empirical field studies I conducted in rural India demonstrate that: (1) individuals do not exhibit a strong tendency to culturally transmit cooperative behaviour (Lamba 2014); (2) behavioural variation observed across human groups is at least in part driven by demographic and ecological processes rather than cultural processes (Lamba \& Mace 2011; 2012; 2013); and (3) individuals are more selfish in larger populations and less likely to copy increasingly cooperative behaviour (Lamba 2014; Lamba \& Mace 2011). Thus, I have tested the first two of the three axioms on which CGS is based, that is, cultural heritability, variation, and competition.

1. People do not tend to copy cooperative behaviour. A central assumption of CGS models is that individuals copy the behaviour of others and acquire cooperative strategies via social learning. I tested this assumption across 14 populations of the same society, the Pahari Korwa of India (Lamba 2014). A series of public goods games, played with real money and for high stakes, across these populations found little evidence that people used either pay-off biased learning or conformity to copy others' cooperative behaviour. The tendency to copy others' behaviour was highly variable and individuals were less likely to copy more cooperative strategies, that is, behaviour that bore higher costs by depositing more money into a group pot. To my knowledge, this is the only quantitative study conducted in real-world populations that tests whether individuals socially transmit cooperative behaviour, and it suggests that they do not. Hence, the only current quantitative empirical evidence testing whether cooperative behaviour is culturally transmitted does not support this idea.

2. Behavioural variation across human groups is at least partly driven by demographic and ecological processes. Richerson et al. state that "it is an empirical matter whether cultural evolutionary processes can maintain substantial between-group variation" (sect. 2.1, para. 3). They cite studies suggesting that behavioural variation across human groups is driven by corresponding variation in cultural norms of cooperation (Henrich et al. 2004; 2006; Herrmann et al. 2008). The studies (Henrich et al. 2004; 2006; 2010a; Herrmann et al. 2008) administered economic games in up to 16 societies and found that game behaviour varied across them. However, they sampled only one or a few populations per society and therefore did not include population (or village) replicates from each society. Thus, they cannot distinguish whether cultural norms or any of the many other variables that vary between the study populations (e.g., population demographics, habitat variation, climate) cause the observed behavioural differences. Building on their work, we administered public goods games, a naturalistic measure of cooperative behaviour involving the sharing of salt (Lamba \& Mace 2011), and ultimatum games (Lamba \& Mace 2013) in up to 21 populations of the same society, the Pahari Korwa of India. For all three measures, we found significant and substantial behavioural variation 
across these multiple populations of the same ethno-linguistic group, and we found that some of this variation was explained by demographic factors such as population size, age, and sex. Hence, individuals from different villages did not share a common cultural norm of cooperation despite their common ethnicity, and their behaviour varied in response to the demographic and ecological features of their environment. Our results are bolstered by similar findings in other societies (Falk \& Zehnder 2013; Gurven 2004; Gurven et al. 2008; Nettle et al. 2011; Tognetti et al. 2012). Since demographic characteristics of populations are likely to change during individuals' lifetimes, we may also expect their behaviour to change in response. And if individuals' cooperativeness is likely to change across their lifetimes, then we should not expect culturally heritable variation in the behaviour of human groups across generations.

3. Individuals are more selfish in larger populations and less likely to copy increasingly costly cooperative behaviour. We found that individuals took more salt for themselves and left less for others in larger villages (Lamba \& Mace 2011). Individuals were also less likely to copy more cooperative behaviour (Lamba 2014). While CGS models invoke cultural transmission to explain the evolution of large-scale cooperation, empirical data suggest that cultural transmission is less likely as behaviour gets more cooperative and cooperation is less likely in large populations.

In conclusion, if individuals do not copy cooperative behaviour, the behavioural variation between groups is unstable due to the demographic changes that groups experience over time, and behaviour is more selfish in larger populations, then we must question the existence of stably transmitted cultural norms of cooperation. There is published empirical evidence which Richerson et al. fail to mention, suggesting that the answer to more than one of their questions may be "no." Therefore, by their own admission, we must also look to hypotheses other than CGS.

\section{The role of cultural group selection in explaining human cooperation is a hard case to prove}

doi:10.1017/S0140525X15000187, e45

Ruth Mace and Antonio S. Silva

Department of Anthropology, University College London, London WC1H OBW, United Kingdom.

r.mace@ucl.ac.uk

http://www.ucl.ac.uk/anthropology/people/academic_staff/r_mace

antonio.silva.09@ucl.ac.uk

http://www.ucl.ac.uk/anthropology/people/graduate_students/a_silva

Abstract: We believe cultural group selection is an elegant theoretical framework to study the evolution of complex human behaviours, including large-scale cooperation. However, the empirical evidence on key theoretical issues-such as levels of within- and between-group variation and effects of intergroup competition - is so far patchy, with no clear case where all the relevant assumptions and predictions of cultural group selection are met, to the exclusion of other explanations.

We believe cultural group selection (CGS) is an elegant theoretical framework for studying the evolution of complex human behaviour. The target article sets out to present evidence that culturally evolutionary processes have resulted in the selection of large-scale cooperation as a group-level adaptation. However, the empirical evidence presented does, in our opinion, still fail to conclusively demonstrate that CGS explains human cooperation better than the competing hypotheses.

Variation in cooperative behaviour-frequently measured through economic games-across different cultures (Henrich et al. 2005; 2006; 2010a) is put forward as evidence that cooperation is a stable, persistent, and group-level trait on which selection at the level of the group could act. However, as we and others have argued elsewhere, high levels of variation in cooperative behaviour are also found within ethnic groups (Lamba \& Mace 2011; 2012; Nettle et al. 2011), suggesting that variation attributed to different cultural norms could in fact reflect individual adaptations to different environments. The $F_{\mathrm{ST}}$ data presented in the article supports the idea that countries differ in some beliefs and values, so at least one of the assumptions of CGS is met. However the beliefs and values examined are not associated with actual cooperative behaviours; for example, demonstrating that there is significant country-level variation in attitudes toward abortion has arguably little relevance to the argument at stake. And has there been enough time for countries to be subject to selection on the basis of this trait?

Cultural difference is one of the foundational assumptions of anthropology, so we should expect to find it. We agree that institutions are likely to be of particular interest in this field and the most promising candidates for cultural traits may be those classic subjects of anthropological investigation such as kinship norms, political systems, and religion, which are generally grouplevel traits, almost by definition. Religion often has the characteristics of a trait designed for group promotion and cohesion (e.g., costly rituals, apostasy punishment, and clear between-group demarcation) making it a strong candidate for spread through intergroup competition. Political systems have to be properties of the group rather than the individual, so group selection should be in operation in these cases (Currie \& Mace 2009; Currie et al 2010). Normative marriage and dispersal strategies may only be effective if the rest of your local population shares your understanding of the rules; strong frequency-dependent selection could maintain variation between groups, so such traits would also be promising cases to seek evidence for CGS. While language is a classic frequency-dependent group trait, the exact design of particular languages is not generally considered to confer any particular benefit over any other, therefore it is unlikely that CGS is operating on languages.

A key aspect of CGS is the idea that traits that provide an advantage to groups, even if at a cost to the individual, are selected for in a situation of intergroup competition. Our attempts at testing this hypothesis for the evolution of parochial altruism the combination of in-group altruism and out-group hostility due to intergroup competition and conflict-using naturalistic field experiments in Northern Ireland, found little evidence to support this idea. Individual and neighbourhood exposure to conflict explained a reduction in out-group cooperation, but crucially had either no effect or also reduced cooperation toward the in-group. In addition, no significant differences in cooperation were found between the two cultural groups when taking into account individual socio-economic characteristics, which in turn best predicted the variation in cooperative behaviour (Silva \& Mace 2014; 2015).

Richerson et al.'s article aims to present the evidence in favour of CGS, hence its adversarial approach rather than the more scientific approach of falsification. However there is still a large empirical project to be done in order to identify the domains in which CGS may be an important evolutionary force. Context is likely to be fundamental, as it is in any evolutionary scenario. Different traits will have different patterns of transmission and different selective forces acting upon them, and benefits may be operationalized at the level of the individual or of the society, or both. We are at a stage of investigation of this topic where we find one specific assumption may be met in one case and another assumption may be met for a different trait or in a different kind of a group; but a consistent story showing the wide applicability of CGS to the evolution of large-scale cooperative behaviour is still lacking, other than at an anecdotal level. 
Commentary/Richerson et al.: Cultural group selection plays an essential role in explaining human cooperation

\section{The disunity of cultural group selection}

doi:10.1017/S0140525X15000199, e46

\section{Olivier Morin}

Konrad Lorenz Institute, 3400 Klosterneuburg, Austria. olivier.morin@kli.ac.at

http://sites.google.com/site/sitedoliviermorin/

Abstract: I argue that demographic selection, migration, and cultural diffusion, three mechanisms of institutional change, have little in common. Two of these lack the key features associated with group selection: they do not present us with group-level selection pressures counteracting individual-level ones, need not produce behavioral altruism, and do not require competition between groups whose members cooperate preferentially with one another. Cultural norms vary, change, and influence cooperation; but that is not group selection.

I agree with Richerson et al.'s stimulating article on its most important point: Human coalitions and institutions have a long cultural history. Their evolution may be driven by three different mechanisms (sect. 2.1.): the differential growth of biological groups; migrations from one group to another; and imitation of norms and other institutional arrangements. The authors' version of cultural group selection (CGS) makes use of all three mechanisms, presenting them as three complementary facets of a unified theory. I will argue that they have little in common (see Fig. 1).

In the first form of CGS distinguished in the target article, groups are defined by three overlapping properties: (i) by descent (you are a group member if you are descended from group members); (ii) by preferential cooperation (group members tend to help one another rather than non-members); and (iii) by cultural links (they make use of the same institutional innovations). In this scenario, you cannot vote with your feet by going to a new group and becoming part of it; neither would you help someone who does not share your values, or fight someone who does. When the last two criteria are relaxed, two new types of CGS result. Type 2 CGS is migration, when individuals vote with their feet to join the societies that offer them the best deal (Boyd \& Richerson 2009). Here, groups are defined by culture and preferential cooperation alone. I am a member of the group of people whose norms I share and whose members I choose to cooperate with-even if I was not born there. Finally, in the third form of CGS (selective imitation) preferential cooperation must also be dropped, as is obvious from Richerson et al.'s logic and examples. Group members are defined by their common adoption of some institutional innovation - that is all: for example, when American firms copy the institutions of German research and development, they do so strictly for their own benefit, not as a way to ally themselves with German industry.

One can use CGS to predict, or explain, two very different things: (1) the evolution of altruistic behavior, and (2) equilibrium selection (André \& Morin 2011; Boyd \& Richerson 1990). Behavioral altruism is an uncompensated sacrifice from the individual to the group, with fitness consequences. Equilibrium selection is a process that favors the most stable forms of cooperation, including non-altruistic, mutually beneficial cooperation. Migration and imitation are more likely to work toward equilibrium selection: We can expect people to join the societies, or adopt the institutions, that give them a better deal. Type 1 CGS is more conducive to true altruism, because it short-cuts adaptive individual decisions. Much of the appeal of CGS is due to Type 1 CGS, and to the promise of finding a scientific rationale for altruism, as a way of backing attacks against standard rational choice theory (Fehr \& Fischbacher 2003; Sober \& Wilson 1999). Yet, much of its plausibility derives from models of the second type, where mutually profitable forms of cooperation spread and no genuine altruism results. The authors' last two types of CGS, thus, do not seem capable of delivering what CGS is widely thought to explain, namely, altruism (André \& Morin 2011; Morin 2014). Tellingly, the authors refrain from making any specific prediction regarding behavioral altruism (sect. 6.2.).

Both Type 2 and Type 3 CGS (migration and imitation) are "driven by human choices" (sect. 2, para. 1), as opposed to the relatively autonomous demographic dynamics that prevail in Type 1 CGS (birth-rates, wars, etc.). Types 2 and 3 of CGS must, at least occasionally, create group-level selection pressures that are fully aligned with individual-level selection pressures, and thus undistinguishable from them (unless we assume that human

\begin{tabular}{|c|c|c|c|c|c|c|}
\hline \multirow{2}{*}{ Type of "CGS" } & \multicolumn{2}{|c|}{ Group membership by... } & \multicolumn{3}{c|}{ Causes and effects of CGS } \\
\cline { 2 - 7 } & $\begin{array}{c}\text { Common } \\
\text { descent? }\end{array}$ & $\begin{array}{c}\text { Preferential } \\
\text { inside-group } \\
\text { cooperation? }\end{array}$ & $\begin{array}{c}\text { Cultural } \\
\text { similarity? }\end{array}$ & $\begin{array}{c}\text { Competing } \\
\text { coalitions? }\end{array}$ & Main causal force & $\begin{array}{c}\text { Resulting } \\
\text { cooperation }\end{array}$ \\
\hline $\begin{array}{c}\text { 1. Demographic } \\
\text { growth and } \\
\text { extinction }\end{array}$ & Yes & Yes & Yes & Yes & $\begin{array}{c}\text { Demography } \\
\text { (fertility, wars) }\end{array}$ & $\begin{array}{c}\text { Altruistic } \\
\text { (or mutually } \\
\text { beneficial) }\end{array}$ \\
\hline $\begin{array}{c}\text { 2. Migrations } \\
\text { between groups }\end{array}$ & No & Yes & Yes & Yes & Individual choices & $\begin{array}{c}\text { Mutually } \\
\text { beneficial }\end{array}$ \\
\hline $\begin{array}{c}\text { 3. Diffusion of } \\
\text { institutions and } \\
\text { norms }\end{array}$ & No & No & Yes & No & $\begin{array}{c}\text { Individual and } \\
\text { collective choices }\end{array}$ & $\begin{array}{c}\text { Mutually } \\
\text { beneficial }\end{array}$ \\
\hline
\end{tabular}

Figure 1 (Morin). Three processes that, for Richerson et al., qualify as "cultural group selection," and my interpretation of the assumptions and consequences behind each. 
decision-making is so maladaptive as to decreases individual fitness systematically). People will vote with their feet: they will join the societies that promise them the best deal. They are likely to adopt institutional innovations that serve everyone's interests. Indeed, most of the examples cited by Richerson et al. concern mutually beneficial forms of cooperation. One can still use groups as units of accounting, but the causality resides entirely in individual choices. Contrary to what is stated in section 4.1, CGS is not, then, characterized by group-level selection pressures counteracting individual-level pressures, pushing individuals to sacrifice their own fitness for the group.

The disunity of CGS is deepened by the authors' inclusion of Type 3 CGS. Cultural diffusion need involve no demographic change, and no change in the composition of coalitions. The manner of cooperation is all that changes. When we use money, majority voting, or the academic practice of research seminars, we are not ipso facto allying ourselves with most of the people who use those practices. A state that decides to allow the legal use of class actions is not forming a coalition with the other states that allow it, or against those that do not. Here, we have sheer equilibrium selection with no differential growth, and no competition between coalitions. Group selection without all this is Hamlet without the prince.

What is wrong with heterogeneous theories? Science needs lumpers as well as splitters; but to test three very different hypotheses as if they were one, is to test only their weakest common denominator. Here, we are left with the uncontroversial view that coalitions or institutions vary and evolve. Richerson et al.'s more substantive suggestion is that useful institutions can only spread through some form of group selection - but to make this point, they dilute the concept of CGS to such an extent that it loses coherence and explanatory heft.

\section{Social selection is a powerful explanation for prosociality}

\section{doi:10.1017/S0140525X15000308, e47}

\section{Randolph M. Nesse}

Center for Evolution and Medicine, Arizona State University, Tempe, AZ 85287-4501.

nesse@asu.edu

http://RandolphNesse.com

Abstract: Cultural group selection helps explain human cooperation, but social selection offers a complementary, more powerful explanation. Just as sexual selection shapes extreme traits that increase matings, social selection shapes extreme traits that make individuals preferred social partners. Self-interested partner choices create strong and possibly runaway selection for prosocial traits, without requiring group selection, kin selection, or reciprocity.

Cultural group selection (CGS) does indeed, as the target article argues, play an important role in explaining human cooperation. However, social selection offers a powerful explanation of extreme human prosociality that contradicts Richerson et al.'s second thesis that only CGS "can easily account for the institutionalized cooperation that characterizes human societies" (sect. 7, para. 2).

The authors document transmissible substantial variations between cultural groups that account for the growth and persistence of some human groups at the expense of other groups. Examples from religious groups confirm the theory. The Shakers had 6,000 members in the mid-19th century but the norm of celibacy shrank the group by outmigration and lack of reproduction, so only one small community remains. In contrast, the Catholic Church's prohibition of birth control fosters group growth.

CGS not only occurs, but also it can act on between-group variations in cooperativeness to help explain human prosociality. Groups with norms enforcing cooperation tend to expand and displace other groups, although, as the target article notes, complexities arise because of the costs of cooperating, the costs of punishing defectors, and movement of individuals and norms between groups. Nonetheless, CGS offers a plausible explanation for cooperation within groups, and tendencies for conflict between groups. Once established, cultural norms for in-group cooperation create forces of natural selection that give advantages to individuals with tendencies to learn and conform to those norms, as well as to their groups, in a recursive cycle that gives rise to capacities for complex culture.

CGS is not, however, the only plausible explanation for extreme human prosociality, and it does little to explain how the process gets going. Social selection can explain extreme human prosocial traits that CGS cannot. This is not Herbert Simon's social selection or that of Joan Roughgarden; it is the idea, first explored in depth in a pair of papers by Mary Jane West-Eberhard, that describe how self-interested social choices create strong selection forces that may account for extreme prosociality and other traits that are otherwise difficult to explain (West-Eberhard 1979; 1983; cf. Nesse 2009). She defines social selection in relation to its exemplar and subtype, sexual selection:

Sexual selection refers to the subset of social competition in which the resource at stake is mates. And social selection is differential reproductive success (ultimately, differential gene replication) due to differential success in social competition, whatever the resource at stake. (WestEberhard 1979, p. 158)

Peahen preferences for peacocks with flamboyant tails give a fitness advantage to males with extreme traits, and to females who prefer males with extreme traits. Positive feedback between selection for the trait and the preference increases the prevalence of alleles that increase mating success until the fitness costs to health and survival outweigh their mating benefits.

West-Eberhard's core insight was that social selection occurs for competition over resources other than matings. A recent review noted, "It would have made as much sense for Darwin (and everybody since) to distinguish between selection by choice versus competition rather than on sexual versus nonsexual selection" (Lyon \& Montgomerie 2012, p. 5). Social selection for competition opens up a whole world of important explanations, but partner choice is more important for explaining cooperation. Despite formal models (Frank 2006; Tanaka 1996; Wolf et al. 1999) and detailed descriptions with examples from many species (Flinn \& Alexander 2007; Lyon \& Montgomerie 2012; Tobias et al. 2012), social selection has been neglected as an explanation for human prosociality.

As many have noted, most social exchanges are mutualisms or are readily explained by kin selection or reciprocity. However, some extreme human prosocial traits still need explanation. CGS has a hard time explaining the pervasiveness and intensity of guilt, motivations for reparations, extreme sensitivity to what others think, concern for other's welfare, pity, commitment, empathy, philanthropy, and pride in generosity. Such traits are, however, expected outcomes from social selection that gives advantages to those preferred as relationship partners or group members.

People prefer partners and group members who have plentiful resources that they generously and reliably share (albeit selectively) with their partners. Thus, to be preferred as a partner requires striving to get resources and a reputation for sharing them. The fitness benefits are not as direct as increased matings, but they are more pervasive. Day by day, reputations and relationships grow and fade as the result of actions small and large. The most desirable relationship partners pair with each other, and this selective association gives them a selective advantage. As West and colleagues note in a critique of trait group selection models, "An alternative is to state as simply as possible what they aremodels of nonrandom assortment of altruistic genes" (West et al. 2007, p. 11). 
Commentary/Richerson et al.: Cultural group selection plays an essential role in explaining human cooperation

The benefits experienced by prosocial individuals, and the benefits of preferring such individuals as partners or group members, shape social traits as extreme and costly as a peacock's tail. This requires no kin selection, reciprocity, group selection, or CGS, although all of those phenomena are also involved in selecting for prosocial traits.

Social selection has been modeled in economic terms as partner choice that shapes advertisements for generosity (Noë et al. 2001) that can become more honest and costly than intended. However, effort invested in carefully assessing partners and making oneself a preferred partner is only the start. Models describing whether to stay or walk away from an existing relationship illustrate how walking away can be inexpensive or even beneficial for the punisher but devastating to the person punished, efficiently enforcing social norms (Aktipis 2004). Shunning and solitary confinement are cruel for good evolutionary reasons.

Careful selection of partners and group members offers little advantage in a species unless differentiated relationships offer a net advantage, some more than others. After a tipping point was reached, however, investments in careful partner choice and traits that make one a preferred partner could coevolve in a possibly runaway process that may have been crucial in making culture possible (Nesse 2010). Social selection may explain how self-interested social choices could have created selection forces by which humans domesticated themselves.

\section{Is cultural group selection enough?}

\author{
doi:10.1017/S0140525X15000205, e48
}

\section{Dwight Read}

Department of Anthropology and Department of Statistics, University of California, Los Angeles, Los Angeles, CA 90095.

dread@anthro.ucla.edu

https://ucla.academia.edu/DwightRead

Abstract: Richerson et al. propose cultural group selection (CGS) as the basis for understanding the evolution of cultural systems. Their proposal does not take into account the nature of cultural idea systems as being constituted at an organizational, rather than an individual level. The sealing partners of the Netsilik Inuit exemplify the problem with their account.

Though recognizing that cultural group selection (CGS) "is not a complete theory of the evolution of cultural variation" (sect. 2.1, para. 5), Richerson et al. consider CGS to be a major player and list four of its prerequisites. All of these are accepted by most anthropologists and sociologists because cultural systems, including norms and institutions, are extended across generations through enculturation, and culture has long been considered to constitute an "extrasomatic adaptation" (White 1959, p. 9), with functional differences the basis for the outcome of competition between groups (see, e.g., Evans-Pritchard's [1940] account of the conflict between the Nuer and the Dinka).

Similarly, the authors' "necessary but not sufficient test of the CGS hypothesis," namely, that "social systems of human societies follow a phylogenetic pattern" (sect. 3.2 , para. 1) is easily passed by cultural and social systems. For example, the historical pattern for the appearance and spread of kinship terminologies as part of the colonization of the Pacific Oceanic Islands, first by Melanesians and then by Polynesians, can be presented as a phylogeny organized around structural differences in the terminologies (Read 2013; see Fig. 1 here). However, these differences do not emerge from changes at the behavioral level assumed by their model of cultural evolution, but are organizational changes. Kinship terminologies, with their algebraic-like structure (Leaf \& Read 2012; Read 1984; 2012; Read et al. 2014) are symbolic, computational systems with an underlying, generative logic and no more emergent from behavior than is arithmetic (contra

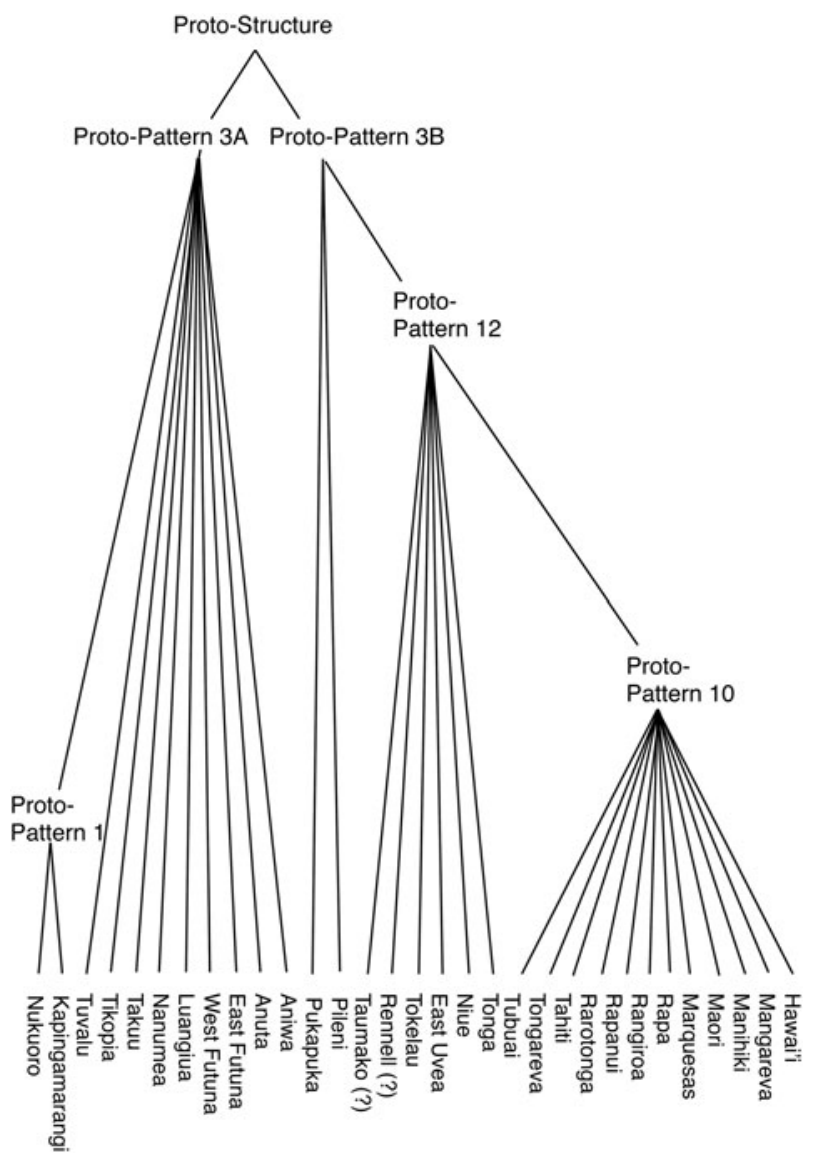

Figure 1 (Read). Phylogeny for Polynesian societies based on the reconstructed sequence of changes in the structural patterns for the sibling kin terms from a sample of the Polynesian kinship terminologies. The specific patterns, based on attributes associated with the sibling kin terms, are discussed in Read (2013). The left-to-right positions of the branch points in the phylogeny correspond to the relative time-sequence of the colonization of the islands corresponding to the societies listed on the right side of the figure (see Figure 7 in Read [2013] for a map showing the colonization sequence). (Reproduced from Read 2013, Figure 9, with permission of the publisher.)

Smaldino 2014)-hence, their evolutionary change is at the global level of organization and not the population level of individual traits (see Lane et al. 2009).

CGS shares with biological group selection the same problem of porous boundaries. If homogenization of between-group traits occurs on a time scale shorter than that needed for the consequences of between-group competition to materialize, then group competition will be obviated. Biological group competition "solves" the porous boundary problem through coupling selection for traits that maintain non-porous boundaries with biological group competition. In extreme form, this leads to the formation of biological species. The functional equivalent for CGS would be a group-level, cultural system with boundaries resistant to the introduction of competing cultural traits.

Consider the cultural adaptation of the Netsilik Inuit of Hudson Bay to Arctic conditions (Balikci 1970). Their adaptation included a culturally prescribed system of sealing partners central to procuring and sharing seal meat in the winter months. The system of sealing partners was but one of several functionally integrated cultural idea systems (see Leaf \& Read 2012) that regulated, among other things, female infanticide, post-marital residence, and preferential cousin marriage. Jointly, these idea systems framed the behavioral patterns and modes of social organization 
necessary for the survival of the Netsilik Inuit under Arctic conditions (Balikci 1970; Read 2005).

The Netsilik adaptation centered on three resources: (1) salmon, (2) caribou, and (3) seals. Seals were obtained in the winter months when a seal surfaced in one of the breathing holes it had to keep open in the pack ice in order to breathe. Because there was no way to know which breathing hole a seal would use, the Netsilik increased substantially their odds of obtaining a seal by stationing about 20 hunters at different breathing holes. Each hunter had 12 sealing partners in the camp, with each partner corresponding to a part of the seal, plus one part of the seal for the hunter and one for his children. When a seal was killed, the hunter's wife distributed the parts of the seal to the wives of the partners in accordance with the part of the seal represented by a partner. The partners were determined at birth by the boy's mother from among distant (cultural) kin-with kin being those recognized by reference through one of their kin terms, such as in the English expression, "he is my uncle."

The system of sealing partners expresses the cultural meaning of being a seal hunter and makes the actions of others, as hunters, predictable, hence forming the basis for cooperation among the sealing partners. In this sense their cultural idea system was a social contract to which the Netsilik adhered. The social contract specified that a seal, through the act of the hunter, became collectively owned by the hunter and his partners, and only they had rights to the seal. Collective ownership was enacted by distribution of the seal meat, based on another cultural idea system that defined a man as the procurer of resources and his wife as manager of the resources he procured $(\operatorname{Read} 2005)$.

Accordingly, the seal was butchered by the hunter's wife and distributed to the wives of the sealing partners. The system of sealing partners was, in effect, the antithesis of sharing through the individual traits of altruism and cooperation since individual benefits arose through collective ownership and rules of sharing expressed through the social contract. From an evolutionary perspective, "it is possible to have a stable social contract for food sharing" even if "the food is implicitly owned by every individual who goes hunting" (Taylor 2014, p. 71).

In their social contract, the Netsilik did not trust a hunter to be altruistic or to voluntarily cooperate, and they instead created an organizational system that depended on neither of these. Cheating, in the sense of opting out of acting in accordance with being a sealing partner, was not a viable option in a context in which individual hunters would likely not survive without averaging hunting risks over a pool of hunters. Consequently, the conceptual boundary for the organization of sealing partners was impervious, given the technologies available to them, to any competing, individual strategy. Cultural idea systems like this operate and evolve at an organizational, rather than an individual, level with the consequence that "human sociocultural organizations ... [have] representations, rules, relationships, management processes and function associated with these organizations, which are different from, and have vastly more transformative and generative capability than, those at the individual level" (Lane et al. 2009, p. 35).

\section{Cultural differentiation does not entail group- level structure: The case for geographically explicit analysis}

\author{
doi:10.1017/S0140525X15000217, e49
}

\footnotetext{
Robert Malcolm Ross ${ }^{\mathrm{a}, \mathrm{b}}$ and Quentin Douglas Atkinson ${ }^{\mathrm{c}, \mathrm{d}}$

${ }^{a}$ Department of Psychology, Royal Holloway, University of London, Egham, Surry TW20 OEX, United Kingdom; ${ }^{\mathrm{b}}$ ARC Centre of Excellence in Cognition and its Disorders, and Department of Cognitive Science, Macquarie University, Sydney, NSW 2109, Australia; ${ }^{\circ}$ Department of Psychology, University of
}

Auckland, Private Bag 92019, Auckland 1142, New Zealand; 'Max Planck Institute for the Science of Human History, D-07745 Jena, Germany. robross45@yahoo.com.au q.atkinson@auckland.ac.nz http://www.ccd.edu.au/people/profile.php?memberlD=595 http://www.fos.auckland.ac.nz/ quentinatkinson/ Quentin_Atkinsons_Website/Home.html

Abstract: Richerson et al. argue that relatively large cultural $F_{\mathrm{ST}}$ values provide evidence for group structure and therefore scope for group selection. However, recent research on spatial patterns of cultural variation demonstrates that, as in the genetic case, apparent group structure can be a consequence of geographic clines, not group barriers. Such a pattern limits the scope for cultural group selection.

Richerson et al. present a wide-ranging synthesis of evidence for cultural group selection. An innovative feature of their argument is that they draw attention to the importance of quantifying the apportionment of cultural variation within and between groups. They calculate $F_{\text {ST }}$ values for 29 aspects of culture between neighbouring groups and argue that relatively large cultural $F_{\mathrm{ST}}$ values provide scope for cultural group selection. In addition, they cite Bell et al.'s (2009) finding that average cultural $F_{\text {ST }}$ values (as indexed by responses to the World Values Survey) are greater for neighbouring groups than average genetic $F_{\mathrm{ST}}$ values as evidence that there is greater potential for cultural group selection than genetic group selection. While we agree that this framework for quantifying cultural variation holds considerable potential, we challenge Richerson et al.'s interpretation of the existing empirical data.

The $F_{\text {ST }}$ statistic belongs to a broad family of "fixation indices" statistics developed by population geneticists to study genetic differentiation between populations (Holsinger \& Weir 2009). Several studies have demonstrated that human genetic variation is predominantly clinal, with differentiation between populations being strongly predicted by geographic distance across a variety of biological markers (Handley et al. 2007). Discontinuities do exist (typically associated with geographic obstacles), but many apparent genetic barriers have proven to be artefacts of heterogeneous spatial sampling (Handley et al. 2007). A clinal pattern of variation is consistent with an "isolation by distance" (IBD) model (Wright 1943), in which individuals tend to migrate limited distances relative to the total geographic range of the species. Under IBD, a gradual blending of one population into the next is predicted, rather than group barriers. Nevertheless, two sampling locations can produce significant $F_{\text {ST }}$ values simply due to their geographic separation.

None of the 29 cultural $F_{\text {ST }}$ estimates reported by Richerson et al. or the cultural $F_{\mathrm{ST}}$ estimates reported by Bell et al. (2009) have been analysed within a spatially explicit framework. This renders these estimates difficult to interpret, since, as in the genetic case, apparent population structure could be an artefact of discontinuous spatial sampling, rather than cultural barriers.

Recently, we published a study that quantified the extent to which geography and group affiliation independently predict cultural differentiation between ethnolinguistic groups (Ross et al. 2013). We used geographic coordinates and coded narrative elements of 700 versions of a folktale from 31 European groups, analysing both individual folktales and group-level differentiation using $\Phi_{\mathrm{ST}}$, a fixation index that is closely related to $F_{\mathrm{ST}}$ (Holsinger \& Weir 2009). We found significant differentiation between groups with an average $\Phi_{\mathrm{ST}}$ of 0.091 , indicating that, on average, $9.1 \%$ of the variation between individual folktales was between groups, which is considerably higher than the genetic differentiation found between comparable European populations (Lao et al. 2008; Novembre et al. 2008). However, incorporating geography into the analysis revealed that at the level of individual folktales, geographic distance explains considerably more variation between folktales than group boundaries (6.6\% of variance vs. $3.7 \%)$. Such a pattern of predominantly clinal variation is consistent with IBD- 
like cultural diffusion processes. This means that geographically close individuals/tales from neighbouring groups tend to be more similar than is suggested by the relatively large average cultural $\Phi_{\mathrm{ST}}$, thereby limiting the scope for cultural group selection.

Two recent studies speak to the generality of our findings and the $F_{\mathrm{ST}}$ estimates from Richerson et al. First, Brown et al. (2014) examined group-level folk song differentiation among nine indigenous ethnolinguistic groups in Taiwan. They found significant cultural differentiation between groups. Intriguingly, however, cultural $\Phi_{\mathrm{ST}}$ was found to be an order of magnitude smaller than genetic $\Phi_{\text {ST }}$ for the same groups, contra Bell et al.'s (2009) hypothesis that human groups generally show a higher degree of cultural differentiation than genetic differentiation. This result is particularly pertinent to discussions about human evolution, because the folk song data are drawn from small-scale indigenous societies whose lifestyles and group structure better approximate those of our ancestors than the large-scale multiethnic nation states studied by Bell et al. (2009). Second, Shennan et al. (2015) examined individual level variation and group-level differentiation in two material culture complexes - pottery and personal ornaments - from 361 sites of 22 putative Neolithic cultural groups in Europe. At the level of individual artefacts, cultural affiliation was an independent predictor of pottery variation, while geography was not; but both cultural affiliation and geography were independent predictors of ornament variation. At the group level, they found significant cultural differentiation, with geography predicting differentiation in ornaments but not pottery.

This result suggests that the relative influence of cultural barriers and geographic effects can vary across different cultural markers in comparable populations, just as is the case for different genetic markers (i.e., autosomal DNA, mitochondrial DNA, and Y-chromosome; Jobling 2012). Caution is therefore warranted in making generalizations about the relationships between groups on the basis of analysis of a limited range of cultural traits.

While we support the rigorous analysis of empirical data to quantify cultural variation, the evidence we present here suggests that cultural differentiation between groups varies considerably across cultural domains and spatial scales and is often best explained in terms of geographic clines - a pattern that suggests IBD-like cultural processes and limited scope for cultural group selection between neighbours. We note that, in the absence of stable individual level trait differences between neighbouring groups, the most important forms of variation for cultural group selection may be group-level traits (Smaldino 2014), such as the presence or absence of particular religious or political institutions, rather than the values, stories, songs, or material possessions of individuals. Further work that examines individual variation and group-level differentiation using a geographically explicit framework across the full spectrum of aspects of human culture is needed.

\section{When is the spread of a cultural trait due to cultural group selection? The case of religious syncretism}

doi:10.1017/S0140525X15000229, e50

\section{Carlos Santana, Raj Patel, Shereen Chang and Michael Weisberg \\ Department of Philosophy, University of Pennsylvania, Philadelphia, PA 19104-6304. \\ csantana@sas.upenn.edu pater@sas.upenn.edu \\ shereenc@sas.upenn.edu weisberg@phil.upenn.edu \\ http://www.phil.upenn.edu/ weisberg/Home.html}

Abstract: The reproduction of cultural systems in cases where cultural group selection may occur is typically incomplete, with only certain cultural traits being adopted by less successful cultural groups. Why a particular trait and not another is transmitted might not be explained by cultural group selection. We explore this issue through the case of religious syncretism.

Richerson et al. give compelling evidence that the conditions for Darwinian evolution are sometimes met in the case of cultural groups. By presenting evidence for cultural group variation, inheritance, and selection, the authors succeed in rendering the cultural group selection (CGS) hypothesis more plausible, and we agree that CGS may partly explain cooperation and other cultural traits. We remain unconvinced, however, that the evidence demonstrates that CGS is a particularly strong force in cultural evolution because selection mechanisms - conquest, imitation, and migration-frequently do not involve selection between cultural groups, but instead work at the level of individual cultural traits.

Consider a case where the conditions favorable to CGS are met: Distinct cultural groups compete, and a group achieves greater success due to cultural variation. According to CGS, we should expect the cultural traits underpinning that group's success to spread. Presumably, such transmission occurs either by complete assimilation to the successful group or by selective adoption of traits, with preference for traits specifically contributing to competitive success.

Total transmission is rarely the case. Even when it is clear that selective conquest, migration, or imitation has occurred, these selective forces rarely involve the total replacement of all the features of the less successful group with all the features of the more successful group. Instead, cultural groups in competitive situations adopt only some features of competing groups, or more frequently, new hybrid cultural traits emerge through the fusion of features from multiple groups. In any particular case, there are various potential reasons for why one trait, and not another, was adopted after a process of conquest, imitation, or migration. While CGS may be a factor, we can also appeal to other factors not accounted for by CGS, including the direct appeal of that trait or pure chance. To be convinced that CGS is a strong force in cultural evolution, we need evidence that cultural borrowing in cases of conquest, imitation, and migration is predominantly driven by contribution to group-level differential success, and not by other potential explanations.

We speculate that CGS is not among the strongest forces driving which cultural traits spread in such cases. To see why, let us examine a paradigmatic case. The authors (Richerson et al.) consider the spread of religion as a flagship case of CGS, noting that features of Christianity contributed to the success of Christian groups, so those cultural features spread both through successful conquest and through comparative success in situations of resource scarcity. A closer inspection, however, calls this story into question. The spread of a religion does not entail the spread of all its cultural features. On the contrary, it has long been recognized that the spread of religion in the face of differential success of cultural groups involves syncretism, defined as "a type of acceptance characterized by the conscious adaptation of an alien form or idea in terms of some indigenous counterpart" instead of the replacement of the indigenous trait by the foreign one (Madsen 1967, p. 369). Consider the spread of Roman Catholicism to what is now Mexico. Invading European cultural groups out-competed the pre-Columbian cultural groups of Mexico, and part of the conquest involved the adoption of Catholicism by native groups. The adoption, however, was syncretic - the surface trappings of Catholicism became part of local culture, but indigenous groups were selective about which Catholic practices they adopted, and also selectively retained native practices by giving them a nominally Catholic guise. Well-known examples include the retention of indigenous holidays such as Día de Muertos, the continuance of traditional practices such as curanderismo, and the thinly-veiled re-presentation of indigenous deities as Catholic saints such as La Virgen de Guadalupe.

How does syncretism of this sort tell against CGS? Syncretism demonstrates that the spread of a religion due to the dominance of a cultural group does not entail the successful reproduction of any 
particular aspect of the cultural systems associated with that group. Although, for instance, Mayan communities adopted the practice of saint worship from the conquering Spanish, Mayan saint worship tends to "evince a more profound parochialism than did saint worship in 16th-century Spain. Cosmologically, Maya saints have become decidedly local personages relatively independent of the remote ... God" (Watanabe 1990, p. 137). This is telling. The conquest of New Spain was facilitated by the parochialism of indigenous cultural groups; the fractious independent city states of Mexico could not compete with a nation-state built around a common identity. Nevertheless, this cooperative feature of Spanish Catholic culture was resisted by many of the conquered peoples, even while cultural traits irrelevant to differential success (e.g., the names of deities) were adopted. In short, the veneer of Catholicism spread, but for CGS, it is not the veneer that matters, but rather the values and practices which contributed to differential group success. Which values and practices were adopted, however, seems to have little to do with whether they contributed to success, but instead with how those practices individually appealed to the conquered peoples.

Other cases of the spread of religion by conquest, imitation, and migration exhibit the same pattern, as do, we would argue, cases of cultural transmission in other domains. To a large degree then, which cultural traits spread in cases of differential competitive success is explained by factors other than the role those traits played in that success. To determine the relative strength of CGS in relation to other cultural evolutionary forces, we need not only to establish that conditions favorable to CGS are met, but also to examine particular instances of cultural transmission. This would involve systematically sampling traits from the more successful cultural system, then determining for each individual sample whether it spread and, if so, the extent to which CGS contributed. By establishing a theoretical background against which we can perform this sort of investigation into the contributions of CGS to the evolution of human cultural traits, Richerson et al. open the doors to research that may help us better understand many of the most notable features of culture.

\section{Cultural group selection in the light of the selection of extended behavioral patterns}

\section{doi:10.1017/S0140525X15000230, e51}

\section{Carsta Simon}

Department of Behavioral Science, Oslo and Akershus University College, 0130 Oslo, Norway.

carsta.simon@hioa.no

https://www.hioa.no/tilsatt/csimon

\begin{abstract}
The cultural group selection hypothesis is supported by considerations of short-term and long-term behavioral patterns of group members, and the short-term and long-term consequences of that behavior. The key to understanding cooperation lies in understanding that the effect of an extended behavioral pattern does not equal-and might even be opposite to - the added effects of its parts.
\end{abstract}

Richerson et al. argue that widespread cooperation between human non-relatives and Darwin's observation that individuals "sacrifice themselves for the common good" (Darwin 1874, pp. 178-79; see epigram at start of target article) are best explained by a mechanism of cultural group selection (CGS). In the following, I elaborate on how ontogenetic mechanisms may also have contributed to our organization in groups and how both cooperation and the sub-phenomenon of "self-sacrifice" may be understood as parts of temporally extended behavioral patterns exhibited either by a group or by individuals.

Behavior of groups, as well as of individuals, takes time. Since time is limited, possible activities compete. Natural selection chooses those activities that correlate either with Phylogenetically
Important Events (PIE) that ultimately enhance fitness or ontogenetic proxies of these PIEs (Baum 2012). Temporally extended activities have parts that consist of smaller-scale behavioral patterns. Thus, natural selection acts on behavioral patterns of varying complexity. Those patterns might be extended in time, as well as in social space; that is, they can be exhibited by an individual or by a group. More local behavioral patterns have other consequences than more extended patterns embracing those local patterns. To estimate an activity's effect on an individual's or a group's fitness, we need to weigh the long-term effects of the sum of local acts against the long-term effect of the behavioral pattern as a whole. The key to understanding cooperation or selfsacrifice lies in the realization that the effect of the pattern as a whole does not equal the added effects of its parts, let alone does a particular part affect fitness in the same direction as the whole pattern. Focusing on the consequences of parts (i.e., single local acts) can lead us up the garden path when explaining cooperation.

The following two examples illustrate how the effect of an individual's local act can differ from the effect of an individual's or a group's extended behavioral pattern: An individual's local act, such as an alcoholic's acceptance of a drink at a party, is promptly followed by relaxed and friendly social interactions, that is, proxies of fitness-enhancing PIEs. We call the acceptance of the drink "impulsive." The alcoholic's refusal of the drink is promptly followed by proxies for fitness-decreasing PIEs such as social disapproval or simply the absence of relaxed social interactions. If an alcoholic refuses a drink, we say that he or she is showing "selfcontrol." Despite the short-term consequences, an extended pattern of self-controlled behavior leads to enhanced fitness. Even if every single refusal of a drink is punished by proxies for fitness-decreasing PIEs, the extended pattern of abstinence from alcohol will correlate with being healthier, having more well-functioning relationships, and a better economy-self-controlled behavior (as an extended pattern) enhances fitness, assessed in the long run (Baum 2015; Rachlin 2004).

A parallel mechanism selects the behavior of a group. Each particular engagement in a cooperative activity, such as recycling, might be locally costly for the individual. If, however, enough group members cooperate, those activities lead to fitness-enhancing PIEs or their proxies. The effect of the more extended behavioral pattern is in the interest of the individual as well as of the group. Of course, those individuals who in fact recycled are not necessarily those whose fitness actually benefits. In most instances, however, the whole group benefits, which means that those who contributed at their own immediate cost often do indeed benefit in the long run. An individual's acts that lead-if they are not punished-to PIEs advantageous for his or her own fitness but disadvantageous for the fitness of other group members, are labeled "selfish," whereas acts that are in the short run costly for the individual but beneficial for the group - including the individual - are called "cooperative." How many instances of "self-sacrifice" are advantageous for the individual's fitness can be understood when analyzing them as parts of rates of cooperative behavior, which correlate with rates of consequences, such as reciprocity.

Even if altruistic behavior is not central to their argument, Richerson et al. "are inclined to think that evolution has built an element of $[\ldots]$ true altruism into our social psychology" (sect. 6.2 , para. 2). If "true altruism" is defined by the absence of fitness-enhancing consequences on the actor's behavior, here, Richerson et al.'s account is not reconcilable with the view of altruistic behavior as a temporally extended behavioral pattern that may be learned over an individual's lifetime (Rachlin 2002). An individual act of cooperation, as well as of altruism, each of which might even be followed by fitness-decreasing PIEs in the short run, can nevertheless be fitness-beneficial when repeated over time. Just as biological selection has acted on groups of organs constituting organisms, extended behavioral patterns (i.e., groups of acts) can be selected together. 
Commentary/Richerson et al.: Cultural group selection plays an essential role in explaining human cooperation

How do the abstract patterns of behavior resist being broken up by selfish behavior?

Richerson et al. posit that group-variable culturally transmitted social norms with group-level functionality are evidence of CGS. Teaching and following norms and rules are practices that function to overcome selfish and impulsive behavior. We have rules such as "brush your teeth twice a day" to ensure that our offspring act in a self-controlled manner and to maximize their fitness in the long run, and we have rules such as "help others" to ensure the coherence of the group.

In different environmental situations, natural selection occurring within groups and between groups may have different relative strengths. Even if cooperation, defined as "working together toward a shared aim" (Wehmeier and Hornby 2000), was selected because it commonly enhanced the individual's long-term fitness, it neither follows that cooperation enhances all cooperators' fitness, nor that all acts that we classify as parts of cooperative behavior do so. A truly self-sacrificing act can come about because it is generally advantageous to cooperate, and making decisions on a case-by-case basis is costly. Just as a single drink in a pattern of abstinence does not significantly change the correlation between that pattern and PIEs (e.g., good health), a single fitnessreducing act of an individual will not significantly change the correlation of cooperative behavior and its fitness-enhancing effect resulting from the group's collaboration.

Richerson et al. offer a coherent framework which is supported, complemented, and potentially slightly modified by the proposed considerations of behavioral patterns and their consequences in different time frames.

\section{ACKNOWLEDGMENT}

I wish to thank William M. Baum, Per Holth, Kalliu Couto, Florian Lange, and Johan Viklund for helpful feedback on earlier drafts of this commentary.

\section{Self-interested agents create, maintain, and modify group-functional culture}

doi:10.1017/S0140525X15000242, e52

\section{Manvir Singh, Luke Glowacki, and Richard W. Wrangham \\ Department of Human Evolutionary Biology, Harvard University, Peabody Museum, Cambridge, MA 02138. \\ manvirsingh@fas.harvard.eduｇlowacki@fas.harvard.edu wrangham@fas.harvard.edu http://www.manvir.org http://scholar.harvard.edu/glowacki/home}

Abstract: We agree that institutions and rules are crucial for explaining human sociality, but we question the claim of there not being "alternatives to CGS [that] can easily account for the institutionalized cooperation that characterizes human societies" (target article, sect. 7). Hypothesizing that self-interested individuals coercively and collaboratively create rules, we propose that agent-based hypotheses offer viable alternatives to cultural group selection (CGS).

Richerson et al. argue that cultural systems, such as prosocial religion and institutions that maintain social order, play a vital role in enabling the cooperation of both small- and large-scale human societies. We agree. But we are unconvinced that cultural group selection (CGS) is the only hypothesis able to account for the existence of such phenomena. CGS leads to group-functional culture by selecting on cultural variation (Boyd \& Richerson 2010), but such selection presupposes that group-functional equilibria exist already, suggesting that other processes, including within-group dynamics, contribute to the emergence of groupfunctional culture. Since researchers have long recognized that individuals with the requisite power coercively and consensually create rules, we propose a complementary "rule-making hypothesis" for group-adaptive institutions. Understanding the contribution of such agent-based social processes is essential for evaluating the value and limits of CGS theory.

Focusing specifically on rules and institutions that control social deviance (one of Richerson et al.'s three examples of group-adaptive culture; see sect. 6.2), we describe two manifestations of this rule-making hypothesis. The first emerges when asymmetries in power or influence allow parties to create and impose rules that satisfy their own interests. Depending on whether the interests of these coercive authorities coincide with those of the group, these individuals will sometimes but not always create and maintain rules with group-level benefits. An alternative form of this hypothesis develops when power is more evenly distributed. In these cases, coordinated groups of individuals can consensually create, modify, and enforce rules that control defection.

Individuals can have key roles in producing group-functional rules if they have sufficient power or influence to institute cultural changes and enforce new rules. Schapera (1970) observed that Tswana tribal chiefs drove cultural change by introducing innovations ranging from single rules, such as the banning of beer, to infrastructural changes, like the establishment of a police system. The chiefs' objectives in creating rules included quelling social disturbance, reducing poverty, and collectivizing otherwise divergent interests - goals with explicit group-level benefits. Functional rules can also stem from leaders dealing with novel forms of conflict. Llewellyn and Hoebel (1941), in their ethnography of the Cheyenne legal system, described how tensions arose after one man borrowed another individual's horse without permission. The military chiefs eased the friction between the parties and then established a rule: "Now we shall make a new rule. There shall be no more borrowing of horses without asking ... [If] the taker tries to keep them, we will give him a whipping” (p. 128). Considering the role of coercive rule-makers offers an alternative explanation not only for the origin of group-functional culture, but also for its maintenance, because powerful rule-makers should continue to enforce group-functional rules as long as the rules satisfy their own perceived self-interest. Moreover, the coercive authority dynamic accounts for the ubiquity of institutions that asymmetrically benefit the powerful, spanning etiquette norms (e.g., Trobriand Islanders: Malinowski 1926) and food taboos (e.g., the Etoro: Kelly 1980; the Aranda: Spencer \& Gillen 1927; and the Sanumá: Taylor 1981).

Coercive rule-makers who have the power to change rules should create group-functional culture when their own interests coincide with those of their groups. In some cases, these overlapping interests stem from coercive rule-makers preferring groups with cooperative, compliant individuals. Elders of the Kurnai of Australia demanded that young boys "listen to, and obey the old men" and "live peaceably with their friends" (Howitt 1885, p. 316), rules that improve the welfare of the elders while also creating group-level benefits. Studies of punishment in animal societies show that CGS is not necessary for the coercive enforcement of group-adaptive behavior (Singh and Boomsma 2015). Reeve (1992) found that naked mole rat queens harass lazy subordinates, while Flack et al. $(2005 ; 2006)$ noted that dominant macaques police conflicts among subordinates, stabilizing their own high status while reducing within-group conflict.

To this point, we have illustrated the ability of powerful individuals to create group-adaptive rules. However, the development of group-functional culture does not require that novelty originate with coercive authorities. Parties of similarly powerful individuals can also establish and maintain rules to control each other's behavior. Systems of rules that emerged across camps during the California Gold Rush represent a well-studied example of self-interested individuals coordinating to create and enforce novel institutions of social order. Despite the absence of organized state law, miners residing at promising digs convened and instituted majority-approved rules to protect mining rights and safety (McDowell 2004). Violations of those rules spurred injured parties to seek redress through the support of the community, who used coordi- 
nated punishment to enforce rules. Ostrom's (1990) review of small-scale institutions that manage common pool resources similarly exemplifies how the coordination of self-interested agents can produce group-functional culture. Fishermen in Alanya, Turkey, for example, took 10 years to perfect an institution to control overharvesting and conflict, with a built-in means of motivating individuals to monitor and enforce violations of mutually recognized rules. Last, group consent can interact with authorities to produce group-functional culture. Among the Enga of New Guinea, leaders presented cultural innovations to their groups, though group consensus ultimately governed adoption. As Wiessner (2002) summarized, "The innovations promoted were ones that leaders felt could be played to their own advantage; the innovations that stuck were those that worked for the individual and the group" (p. 251).

Here we briefly reviewed evidence that individuals motivated by self-interest can create group-functional culture through coercion, consensus, or both. Although our discussion is largely restricted to rules controlling deviance, these arguments apply to the development of other domains of group-functional culture as well, such as institutions that reward otherwise costly behaviors (Glowacki \& Wrangham 2013) and prosocial religion (Norenzayan et al. 2016). Only after recognizing complementary and alternative hypotheses for group-functional culture, as well as the criteria for testing among them, will we be able to assess Richerson et al.'s claim that CGS is uniquely important in generating the cultural systems underlying human cooperation.

\section{The selective social learner as an agent of cultural group selection}

\author{
doi:10.1017/S0140525X15000254, e53
}

\section{Sarah Suárez and Melissa Koenig \\ Institute of Child Development, University of Minnesota, Minneapolis, MN 55436. \\ suare062@umn.edu mkoenig@umn.edu \\ http://www.cehd.umn.edu/icd/people/faculty/cpsy/koenig.html}

\begin{abstract}
Developmental research characterizes even the youngest learners as critical and selective, capable of preserving or culling cultural information on the bases of informant accuracy, reasoning, or coherence. We suggest that Richerson et al. adjust their account of social learning in cultural group selection (CGS) by taking into consideration the role of the selective learner in the cultural inheritance system.
\end{abstract}

Richerson et al.'s discussion rightly characterizes social learning as one of the key facilitators of cultural group selection (CGS). While we agree with the idea that social learning is essential for cultural inheritance, and serves as a source of cultural variation and selection, we believe that the authors' discussion has underspecified the significant role of the rational, critical, and selective social learner in these evolutionary processes.

We would first like to note that behavioral imitation, which the authors focus on, is not the only form of social learning that could reduce intergroup cultural variation. Cultural variation between groups is a starting point not only for the spread of group-beneficial behaviors but also for group-beneficial beliefs, ideas, or concepts. Contact with out-group scientific knowledge, for example, could result in the adoption of this cultural information by less successful groups. In such cases, learning would not be primarily imitative in nature - one could not describe cross-cultural learning about microbes, for example, as primarily imitative in nature, and yet this has reduced between-group variation in cultural beliefs about the unseen causes of disease.

That said, the selective social learner is influenced by factors beyond those cited by Richerson et al. (those being a penchant for conformity, the sway of prestige, affinity for cultural norms, or group membership biases). A growing body of developmental research characterizes even the youngest social learners as active participants in the learning process, whose selectivity can act to preserve or cull testimonial information on the basis of its accuracy, support, and coherence with other beliefs (Stephens et al. 2015). These belief-formation processes are supported by children's rapid assessments of others' competence, expertise and trustworthiness. Monitoring the content and coherence of messages protects learners against misinformation, alerting them to conflicts between incoming messages and their already established knowledge and beliefs (Mercier \& Sperber 2011). For example, infants detect overt labeling errors and anomalous referential actions (Gliga \& Csibra 2009). They expect humans with appropriate information to speak truthfully, and actively correct inaccurate labelers (Koenig \& Echols 2003). In imitation research, children have been found to give priority to the approach taken by a successful individual over an unsuccessful group, showing that in learning actions, successful outcomes often trump conventional means (Scofield et al. 2013; Seston \& Kelemen 2014; Wilks et al. 2015). Further research suggests that the detection of inaccuracy might lead to enhanced memory for incompetent sources and poor memory for the information they present (Corriveau \& Harris 2009; Koenig \& Woodward 2010; Sabbagh \& Shafman 2009).

Even more relevant to CGS, research in this field suggests that children make rational learning decisions on the basis of whether or not the messages they receive from informants violate their existing understanding of the world (Sobel \& Kushnir 2013). Preschoolers, for example, have been known to preferentially learn from more expert (Koenig \& Harris 2005; Koenig \& Jaswal 2011), more grammatically proficient (Corriveau et al. 2011; Sobel \& Macris 2013), and logically consistent informants (Doebel et al. 2011). They also flexibly adjust their trust in informants' testimony based on their understanding of what is improbable and impossible (Corriveau et al. 2014; Shtulman \& Carey 2007; Woolley \& Ghossainy 2013). Young learners' reliance on their own understanding of what is correct, true, or possible should not be underestimated: Their preferences for native-accented speakers (Corriveau et al. 2013), adults (Jaswal \& Neely 2006), and familiar speakers (Corriveau \& Harris 2009) are reversed if they learn that such sources have proven inaccurate. This is not to say that children are always critical in the face of message conflicts: Preschoolers have been found to trust a deceptive adult who provides inaccurate information about an object's location (Jaswal 2010; Jaswal et al. 2010). Interestingly, this may be because preschoolers are more sensitive to violations of commonly held cultural knowledge (Stephens \& Koenig 2015; Koenig \& Stephens 2014).

As children develop, they incorporate their growing knowledge and experience about speakers and the world to evaluate new information. Considerations about message coherence and plausibility are taken into account along with other factors - including, as Richerson et al. mention, informant prestige, cultural conventions, conformity, normativity, group membership, and so on. We suspect that children appraise social learning situations based on their recognition of both cultural and epistemic norms, with priority afforded to each based on the situation (Hodges 2014), type of testimony or information being exchanged (Stephens \& Koenig 2015), and the values endorsed in their environment (Reifen Tagar et al. 2014).

How exactly does this selective learner play a role in CGS? Richerson et al. describe three forms of social learning that help maintain or reduce group-level cultural variation: (1) accurate, rapid social learning; (2) conformist social learning, described as mechanisms that maintain intergroup variation of cultural norms and behaviors; and (3) selective imitation of cultural behaviors, described as a process by which intergroup variation is reduced. Along these lines, we would argue that the selective learner's ability to critically evaluate cultural information on the basis of its accuracy, support, and coherence contributes to CGS in two main ways. First, as previously described, the social learner can 
reduce intergroup cultural variation by rationally evaluating outgroup cultural information and adopting that which is deemed to be not only useful, but reliable. Second, the selective social learner is able to ensure accurate cultural transmission not only through imitative or conformist strategies, but also by evaluating various forms of cultural information on the bases of accuracy, logic, and internal coherence. Thus, when a 16-month-old corrects an informant who labels a shoe as a ball, upholding culturally specific labels, what may appear to be an early tendency toward conformity may involve critical appraisals of messages, along with epistemic inferences about the informant.

In sum, we suggest that Richerson and colleagues take into further consideration the role of the rational and selective social learner, who can critically evaluate cultural information and adjust their own learning accordingly.

\section{Human cooperation shows the distinctive signatures of adaptations to small-scale social life}

doi:10.1017/S0140525X15000266, e54

\author{
John Tooby ${ }^{\mathrm{a}}$ and Leda Cosmides ${ }^{\mathrm{b}}$ \\ ${ }^{a}$ Center for Evolutionary Psychology, and Department of Anthropology, \\ University of California, Santa Barbara, Santa Barbara, CA 93106; ' ${ }^{\mathrm{C} C e n t e r}$ for \\ Evolutionary Psychology, and Department of Psychology and Brain Sciences, \\ University of California, Santa Barbara, Santa Barbara, CA 93106. \\ tooby@anth.ucsb.edu \\ cosmides@psych.ucsb.edu \\ www.cep.ucsb.edu
}

\begin{abstract}
The properties of individual carbon atoms allow them to chain into complex molecules of immense length. They are not limited to structures involving only a few atoms. The design features of our evolved neural adaptations appear similarly extensible. Individuals with forager brains can link themselves together into unprecedentedly large cooperative structures without the need for large group-beneficial modifications to evolved human design. Roles need only be intelligible to our social program logic, and judged better than alternatives.
\end{abstract}

The title of the target article advances the bold claim that: "Cultural group selection [CGS] plays an essential role in explaining human cooperation." By the end of the target article, the argument has been watered down: "evidence ... [justifies] taking the CGS hypothesis seriously as [one] basic explanation" (sect. 7, para. 2 , emphasis added). That is, vaguely characterized nonquantitative facts about humans do not prove the impossibility of CGS influencing the distribution of cooperative behavior, however minutely. We agree it is possible. Richerson et al. similarly retreat from CGS being an essential explanation for human cooperation, substituting the claim that it is an explanation for "our species' highly unusual ability to create large societies with widespread cooperation" (sect. 7, para. 2, emphasis added).

Because "large societies" are a recent evolutionary novelty, it follows that selection in ancestral small-scale societies was what predominantly forged the (genetically) evolved mechanisms that make cooperation in modern large-scale societies possible. Indeed, the context in which something evolves (e.g., cooperative mechanisms in foragers living in small-scale societies) does not predict its capacity to scale (e.g., cooperation in mass societies). Vision that evolved to see things closer than 3 miles can see for light-years; language capacities that evolved to allow our ancestors to speak to hundreds now allow us to speak to millions.

CGS proponents find the existence of cooperation in mass societies a self-evident evolutionary puzzle because the numbers involved evoke the impression that selection is not at equilibrium (which it need not be). In contrast, we think researchers need to carefully characterize the computational architectures of our evolved array of neural adaptations for exchange, delayed implicit reciprocity, risk-pooling, alliances, coalitions, coordination (such as theory of mind), bargaining, aggression, mateship, parenting, kin selection, partner choice, reputation, externality-management, social learning, and so on, together with their interactions. Only then can you know whether any puzzling residue of "group-beneficial behavior" in modern societies remains, requiring further explanation.

The dazzlingly extended forms of modern cooperation we see today (Adam Smith's division of labor supporting globe-spanning trade) appear differentially built out of adaptations for small-scale sociality that modularly scale, such as exchange - rather than the marginal benevolence of Smith's butcher, brewer, and baker. Evidence indicates that political attitudes toward welfare and redistribution reflect a specialized forager psychology of sharing for variance reduction (Petersen et al. 2012) and resource-conflict (Petersen et al. 2013). Societies that attempted to harness general benevolence to organize institutions and production - the USSR, East Germany, China, Cambodia, North Korea, Cuba - were spectacular cooperative failures. That they functioned at all depended on other scalable small-scale specializations - aggressive threats (conditional punishment), hierarchy, dominance, coalitions, and so forth.

Even ancestral foragers had institutions (enduring coordination with different roles). We do not understand why individually selected psychological adaptations for cooperation, coordination, coalitions, theory of mind, metarepresentations (i.e., $x$ is a rule), intelligent instrumental reasoning (that allows locally contingent tailoring of actions to goals), social learning, a social psychology that understands and deploys incentives, hierarchies (and so on) are considered inadequate to explain institutions, then or now. It is puzzling why the authors believe that modern institutions cannot be far better explained without recourse to CGS, by the combined operation of these neural adaptations in dense, persisting social networks of intelligent, cultural agents (Boyer \& Petersen 2011). When the interlinked cognitive niche adaptations (Tooby \& DeVore 1987; see also, Pinker 2010) such as intelligence, language, and culture are added, it is difficult to see any obvious cooperative anomalies.

Gene-culture coevolution proponents claim to see overwhelming evidence of group-beneficial, individually costly behaviors in large societies that cannot be explained by (their computationally impoverished models of) reciprocity. For example, many results are interpreted as showing prosocial, other-regarding preferences purportedly inconsistent with individual selection, including a taste for fairness, excess generosity, and a failure to uniformly act with short-run selfishness. These preferences, together with a taste for altruistically punishing fairness norm-violators, are believed to work together to make people sacrifice their individual interests for the benefit of the group - which then helps groups in intergroup competition. However, the supporting experimental findings typically involve constrained choices that conflate hypotheses, rather than test them cleanly. When these defects are removed from experimental designs, supporting results collapse. For example, when subjects have the added choice of taking from others as well as giving, they no longer give in dictator games (List 2007). Young children, purportedly averse to unfair divisions, will choose to pay a cost to reduce the welfare of others when given the chance (Sheskin et al. 2014). In previous experimental designs testing for third-party punishment of unfair dividers, the only choices available were to punish or not. When this demand-characteristic is removed by adding the option of rewarding unfairness, average "altruistic" punishment approaches zero (Pedersen et al. 2013). Where partners can defect on both the subject and third parties, subjects punish those who defect on them personally (Krasnow et al. 2012). Moreover, they only punish those they subsequently choose to interact with, not those who could only harm others. This indicates that punishment is a tool of negotiation, and not primarily designed to altruistically uphold group norms.

Finally, the models typically used to represent individually selected strategies of social interaction ("reciprocity") are strikingly 
impoverished (e.g., Cooperate, Defect), are largely free of social ecology and computation, and rest on many implausible assumptions. They need to be replaced with an adaptationist game theory. For example, to determine whether to cooperate, agents need to know whether an interaction is one-shot or repeated. When this aspect of real-world cooperation is added to simulations of reciprocity, then "excess" generosity reliably coevolves with reciprocity, eliminating observed generosity as an evolutionary puzzle (Delton et al. 2011). The rapid cultural dynamics of moral norms (think French Revolution) seem far better explained by rivalrous agents opportunistically seeking allies to jockey for self-advantageous norms (Tooby \& Cosmides 2010), than by the slow accumulation of group-benefiting norms through some groups doing better than others. In 1789, institutions that developed over a thousand years were swept away in a thousand days. Thus, we may find that the code and open parameters of evolved programs underlie the combinatorial rules and building blocks of institutional cooperation.

\section{Cultural group selection is plausible, but the predictions of its hypotheses should be tested with real-world data}

doi:10.1017/S0140525X15000278, e55

\section{Peter Turchin ${ }^{a}$ and Thomas E. Currie ${ }^{b}$ \\ ${ }^{a}$ Department of Ecology and Evolutionary Biology, University of Connecticut,

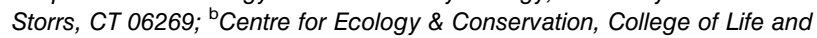 Environmental Sciences, University of Exeter, Penryn Campus, Cornwall, TR10 9FE, United Kingdom. \\ peter.turchin@uconn.edu T.Currie@exeter.ac.uk http://cliodynamics.info \\ http://biosciences.exeter.ac.uk/staff/index.php?web_id=Thomas_Currie}

Abstract: The evidence compiled in the target article demonstrates that the assumptions of cultural group selection (CGS) theory are often met, and it is therefore a useful framework for generating plausible hypotheses. However, more can be said about how we can test the predictions of CGS hypotheses against competing explanations using historical, archaeological, and anthropological data.

Scientific theories such as cultural group selection (CGS) must be assessed in two ways. First, the basic underlying assumptions on which an idea rests should be shown to be coherent and realistic. Second, the theory should generate hypotheses with testable predictions about phenomena in the real world that we should observe if the theory is correct. In the target article, Richerson et al. do an excellent job of demonstrating that, indeed, the assumptions of CGS theory are often met, and it is therefore a useful framework for generating plausible hypotheses. In particular, the properties of cultural inheritance, processes such as conformity and frequency dependence, and the ubiquity and importance of institutions enable the maintenance of variation between groups upon which selection can act even in the face of physical migration between groups (a large hurdle facing genetic group selection models). The importance of this should not be underestimated, given the somewhat controversial and divisive history of this subject.

However, we feel there is more that can be said about how we can test the predictions of CGS hypotheses as explanations of human cooperation, specifically with respect to testing them against competing explanations. CGS is an overarching framework that can generate more specific hypotheses that can be tested against alternatives. Let's consider the following: over the last 10,000 years, the scale of human cooperation has increased by several orders of magnitude: from small-scale groups of some hundreds of foragers to large modern states with populations of hundreds of millions. Social scientists have advanced a multitude of theories explaining this "major evolutionary transition" (sensu Maynard Smith \& Szathmáry 1995). Such theories tend to come in several flavors (Carballo et al. 2014). "Functionalist" (or "voluntaristic") explanations emphasize benefits of cooperation to all: buffering environmental risk, managing competition and efficient allocation of resources, producing public goods such as an irrigation system, and capturing returns to scale in, for example, economic production (Johnson \& Earle 2000). In contrast, "conflict" explanations focus on the dark side of large-scale sociality: class struggle and exploitation, warfare and conquest (e.g., Carneiro 1970). CGS theory can combine these functionalist and conflict elements, but in a highly specific way: Cooperation within societies evolves as a result of conflict and competition between societies.

It is possible (indeed likely) that the best explanatory model will combine more than one mechanism, with different factors, perhaps, interacting in nonlinear, synergistic ways. Evaluation of such complex quantitative explanations is not a problem for modern methods of analysis, especially when combined with a program of building mathematical models that explicitly incorporate such interactions. In our own research we have made a number of steps in this direction. In a recent paper (Turchin et al. 2013) we examined whether increased competition between groups due to the development of horse-based forms of warfare (i.e., involving chariots, cavalry, etc.) was an important force in the historical emergence of very large-scale human societies ("empires"). Following the logic of CGS (or multi-level selection more generally), we constructed an agent-based computer simulation in which "cooperative" cultural traits were only selected for due to the beneficial effects they had in competition between groups (without between-group competition, there was a heavy bias against developing such traits). We were able to test the predictions of this model against historical data about the spatial distribution of empires over a 3,000-year period. Encouragingly, the predictions of the model showed a good match to the real data. Furthermore, turning off some of the important parameters in the models produced a large drop-off in the match between simulations and data. This indicates that our hypothesis is at least a plausible explanation for the evolution of socio-political complexity. This model is admittedly a gross simplification of the actual historical process, and these results are still somewhat preliminary; however, this work does demonstrate the ability to quantitatively test the predictions of hypotheses informed by CGS, using the empirical record of past human societies.

The next step is to test this hypothesis more explicitly against other alternative explanations, including those not motivated by CGS. An important point here is that different theories make very different predictions as to where, when, and under what circumstances we should see the rise of large-scale societies in the archaeological and historical record, and such things as the order in which different aspects of societies emerge. So far the progress in testing such theories has been slow. Yet the huge corpus of historical and archaeological information provides us with a remarkable empirical resource for testing theories and rejecting empirically inadequate explanations. The key is transforming the wealth of information into a systematic form that facilitates the kinds of analyses we described above. Currently, we are collaborating with colleagues from across multiple disciplines and around the world to develop a databank of coded and quantitative historical and archaeological information about past societies (Seshat: Global History Databank: http://seshatdatabank.info/), with which hypotheses about cultural evolution and human history can be tested, including those informed by CGS theory (Turchin et al. 2015). For example, in one project we are assessing the idea that competition between groups led to increased egalitarianism in human groups, particularly beginning with developments of several "axial-age" religions (Bellah 2011). Importantly, this idea will be rigorously tested against other competing explanations, for example, the idea that religion is the "opiate of the masses," by which elites keep the majority of the population 
subservient. Rather than the common approach of the social sciences or humanities where the world is interpreted according to a particular theoretical perspective, we follow the approach in which it is ultimately the data that decide which hypothesis provides the best explanation.

In summary, we contend that CGS has been shown to be a very productive generator of testable hypotheses. This theoretical framework is capable of producing novel, even unexpected predictions that can be then contrasted with predictions made by alternative theories using historical and archaeological data. Whether hypotheses derived from CGS theory are "true" or not, this framework has already demonstrated its value as a productive research program in the sense of the philosopher of science Imre Lakatos (1978).

\section{Multi-level selection, social signaling, and the evolution of human suffering gestures: The example of pain behaviors}

\section{doi:10.1017/S0140525X1500028X, e56}

\section{Jacob M. Vigil and Eric Kruger \\ Department of Psychology, University of New Mexico, Albuquerque, NM, 87131-1161. \\ vigilJ@unm.edu \\ esk@unm.edu \\ http://www.unm.edu/ psych/faculty/sm_vigil.html}

\begin{abstract}
Pain suffering has been naturally selected to be experienced and expressed within a wider social system. The communication of pain improves group coordination and decision-making about engaging in resource dependent and potentially risky endeavors. Recent findings warrant the development of a cohesive framework for understanding the reciprocal nature of pain expression and individual and group-level outcomes that can generate novel predictions on the heuristical expression of human suffering in naturalistic and clinical settings.
\end{abstract}

Richerson et al. present an interesting take on the contemporary movement to understand the complexity of human behavior via multi-level selection forces. Their arguments in favor of multilevel selection by way of cultural group fitness, though speculative, highlight the potential application in evaluating the social structure as a potent mediator in the selection of characteristics of human psychology. It may in fact be the case that a multitude of basic biological systems have been influenced by both individual- and group-level selection forces due to the reciprocal nature on individual and group fitness outcomes. One domain of mental and physical health functioning that likely evolved within the constraints of embedded social communication is the experience and demonstration of visceral suffering in its various forms (e.g., anxiety, sadness) including pain. In this commentary, we propose to extend Richerson et al.'s thesis by showing how human pain perception could have been influenced by multilevel selection, a thesis that, if true, has broad implications for the future directions of evolutionary psychology and the clinical health sciences.

There is no doubt that the behavioral expression of physical discomfort has both intra- and interpersonal functions that directly impact the individual's fitness. Some intrapersonal functions of pain perception include the ability to detect and discriminate environmental threats, to serve as a warning system, to promote selfawareness (e.g., to attend to and protect an injured body-part), and to facilitate operant learning (e.g., to avoid dangerous stimuli; Eccleston \& Crombez 1999). Relative to other species, these intrapersonal functions alone do not explain why humans express so many and such varied non-vital suffering behaviors, including those associated with chronic, psychosomatic, and emotion-induced pain conditions. One likely explanation for the adaptive benefits of pain expression lies in the social-signaling properties of pain behaviors (Vigil \& Strenth 2014).

The social-signaling perspective maintains that pain behaviors are a pinnacle demonstration of vulnerability, which humans use and direct toward selective social partners to gain logistical and emotional social support in times of need (Vigil 2009). Expressive pain behaviors also allow sufferers to assess the altruistic reliability of the targets of their expression (e.g., friends, domestic partners or health care providers). In other words, persons who respond to a solicitation evoked by a pain expression are perceived as potentially more reliable social partners. To date, this social-signaling perspective of pain perception has been used (with relative success) for predicting individual differences that influence the expression of pain in various social contexts, such as audience characteristics (Vigil \& Alcock 2014; Vigil \& Coulombe 2011; Vigil et al. 2014b) and the simulated presence of another person (e.g., the sound of a stranger's voice; see Vigil et al. 2014d).

Still, there is no reason to assume that the social-signaling functions of pain are limited to individual-level fitness incentives alone. Thus, additional selection forces acting at the group level may have played a secondary or coevolutionary role in the selection of pain's perception and expression. As Richerson et al. and others have noted (Geary 2010; Geary et al. 2003), humans evolved in an environment of coalitional competition whereby social cooperation was used to form alliances for protecting oneself from and competing against rival groups for control of ecologically relevant resources (e.g., food and mates). The coordination of group activities was such a predominant feature throughout human evolution that it may make sense to conceptualize the group as a valid unit of selection. This is especially true if the success of group activities depends on communication among members. Given that the expression of pain can be understood as an individual's perception of threat and state of debilitation, the aggregate effect of multiple individuals expressing pain should ultimately influence a group's behavior. Based on this, the selection of individual characteristics and attributes that bolster selective communication of pain within a group should enable improved group coordination and decision-making. Likewise, groups that are better able to assess external threats (predators, neighboring groups, or parasites) and in-group capabilities should have a greater chance of survival. From an evolutionary perspective, this hypothesis makes intuitive sense, given that humans are the quintessential social animal and competition between groups is dependent on achieving effective group-level consensus on resource expenditure and risk-taking (e.g., engaging in new hunting tactics, migration, coalitional warfare).

Complementary to this hypothesis is the role that empathy plays during interpersonal communication. Expressed empathy could be understood as a key behavioral mediator subserving the interpretation of individual-level vulnerability and threat (i.e., pain) in coalition members. Said differently, for pain's expression to find an effective target, the receiver must be able to internalize the current state (and changes in that state) of physical and mental prowess of the signaler. This combination of pain and empathetic signaling (e.g., behavioral mimicry) distributed among group members enables group coordination. This hypothesis is supported by recent findings suggesting that people express empathy systematically with the formation of social identities creating an intragroup empathy bias-the increasing empathy between in-group members greater than that of out-group members (Cikara et al. 2011; 2014). The mutual expression of pain and empathy enables in-group members to use this information in making effective decisions. In line with this, our lab has found that momentary pain reporting is influenced by the quantity and quality of the individual's peer relationships (Vigil et al. 2013), relationships with pair-bonding partners (Vigil et al. 2014c), and other types of co-residents (Vigil et al. 2014a). These findings suggest that pain has been naturally selected to be expressed systematically within a broader social networking system. Further, recent work has explicated a neurobiological relationship 
between social and physical pain. Panksepp (1998) originally proposed the idea of social pain, and since then, numerous studies have demonstrated a cognitive and behavioral overlap between the shared aspects of physical and social pain (Eisenberger \& Cole 2012; Macdonald \& Leary 2005).

On the balance, the diverse research findings from numerous lines of inquiry suggests a need for further empirical and experimental studies that would add to a developing framework for understanding how individual behavior is reciprocally shaped by both individual and group outcomes. Pain behaviors enjoy a rich variability that may not be attributed to individual factors alone, and this new framework may offer alternative means for generating predictions of the evolved nature of human suffering.

\section{Clarifying the time frame and units of selection in the cultural group selection hypothesis}

\author{
doi:10.1017/S0140525X15000291, e57
}

\author{
Andrew Whiten ${ }^{\mathrm{a}}$ and David Erdal ${ }^{\mathrm{b}}$ \\ ${ }^{a}$ Centre for Social Learning and Cognitive Evolution, School of Psychology \& \\ Neuroscience, University of St. Andrews, St. Andrews KY16 9JP, United \\ Kingdom; ' School of Management, University of St. Andrews, St. Andrews, \\ KY16 9RJ, United Kingdom. \\ aw2@st-andrews.ac.uk david@erdal.org.uk \\ www.st-andrews.ac.uk/profile/aw2
}

\begin{abstract}
We raise and discuss two issues for clarification. First, over what timescale is cultural group selection proposed to have been active? Unprecedented cooperation is inferred to have arisen in hunter-gather life well before the recent period on which Richerson et al. focus. Second, what is the unit of selection? Groups (of human individuals)? Or cultural entities along the lines of "meme-complexes"?
\end{abstract}

We congratulate authors Richerson et al. on the scope and scale of the wide-ranging literature they have brought to bear on their thesis. This offers an impressive scholarly review of evidence bearing on the nature of human cultural evolution. Nevertheless, we find it difficult to judge yet how compelling is the resulting case for cultural group selection (CGS) specifically, because of a lack of clarity on some core issues. Here we focus on two.

The first concerns the period of human evolution that Richerson et al. aspire to address, which remains surprisingly ambiguous in the target article. Much of the article focuses on recent times, notably the Holocene. At one point they speculate that CGS may have been important "as far back" as symbolic evolution developments, at around 160 k.y.a. But given that the authors start their article by taking the distinctiveness of human cooperation as the remarkable phenomenon they wish to explain, it seems to us that they should be more seriously addressing the earlier periods of hunting and gathering, including necessarily highly cooperative big-game hunting, which a variety of evidence suggests was already significant between 500 k.y.a. and 1 m.y.a. at least (Whiten \& Erdal 2012). This is important not only because of its relevance to the authors' central concerns, but also because the socio-cognitive processes selected for over this vast timescale are likely to have left a significant legacy in the architecture of the human mind, including its cooperative and cultural dispositions.

The ethnographic, archaeological, and comparative evidence that over the years we (Erdal \& Whiten 1996; Whiten \& Erdal 2012) and others have marshalled indicate that this long hunter-gatherer era was already marked by unprecedented egalitarianism, generalized sharing, and coupled, enhanced levels of cooperation - the core phenomenon the authors profess to wish to explain - of various kinds. These have included cooperation in each of the enterprises of hunting (including manufacturing the weapons and traps utilized) and gathering, but also the ways in which these activities, typically undertaken through division of labour between separately foraging parties, are integrated over space and time and coordinated to permit generalized sharing of spoils at campsites, coupled with information exchange between hunters and gatherers that enhances subsequent foraging effectiveness. Among the many other forms of enhanced cooperation are shared child care and inter-band marriage arrangements. The result is that interdependence in hunter-gatherers displays unique levels and forms among primates, such that through the exploitation of its socio-cognitive niche, the band effectively acts like a unified predatory organism, able to outcompete such "professional predators" as the large social carnivores of Africa. It is thus the earlier evolution of these distinctive cooperative and other characteristics, in small bands numbering about 30 individuals, that begs explanation. Given these were surely already enmeshed in complex cumulative cultures, shouldn't the analysis of Richerson et al. be focused more on the social dynamics and selective regimes that have operated in tens of thousands of generations of hunting and gathering?

The unique forms and degree of interdependence among the members of a human hunter-gatherer band-which mean that the inclusive fitness of individuals is highly contingent on their contributions to the effectiveness of the group as a whole-could perhaps be argued to provide an unusually supportive context for the operation of genetic group selection. However, we are here talking instead of cultural group selection, and this leads us to the second issue we wish to raise, which is the more general one of what exactly are the replicating (even if with varying fidelity) entities that are proposed to be exposed to differential selection in the CGS theory? We feel this remains surprisingly unclear in this article. At times the authors write as if it is cultural "groups" of people, with some groups outcompeting or absorbing others. But should not the unit of replication instead be a cultural entity, more along the lines of (although not necessarily isomorphic with) - what have earlier been called "memes" or "meme-complexes" (e.g., Aunger 2000)? The latter concept of a set of co-adapted cultural elements (Dawkins 1976, pp. 212-213) is presumably where the "group" aspect of CGS would get purchase? And for CGS to occur, these meme-complexes would somehow need to spread preferentially across populations, as when cultural complexes such as religions like Catholicism, or even national cultures in the case of large scale invasions like that of the Roman Empire, spread through new populations. Selection involving the differential reproductive success of cultural entities (group-level selection where "group" is akin to "meme-complex") is a very different phenomenon to selection involving differential reproductive success of groups of individuals. The two are not mutually exclusive but it needs to be clearer whether CGS is conceptualized as involving both types of competition or one in particular?

If the focus of the "group" term in CGS is cultural complexes, perhaps such phenomena have indeed characterized only the relatively recent phases of human cultural evolution that the authors focus on, particularly the Holocene. But is such a conclusion based only on the fact that we have rich, relevant evidence about cultural change across this recent period? We know all too little about the processes that characterized the earlier and vastly longer period of our hunter-gatherer history we alluded to earlier. Indeed, we know all too little about the nature of cultural change from all the studies undertaken in the recent ethnographic past. From what we do know (e.g., Hewlett et al. 2011; Jordan 2015), it is far from apparent that "group" needs inserting in "cultural selection" to characterize the processes at work. 
Response/Richerson et al.: Cultural group selection plays an essential role in explaining human cooperation

\section{Authors' Response}

\section{Cultural group selection follows Darwin's classic syllogism for the operation of selection}

\author{
doi:10.1017/S0140525X15000606, e58
}

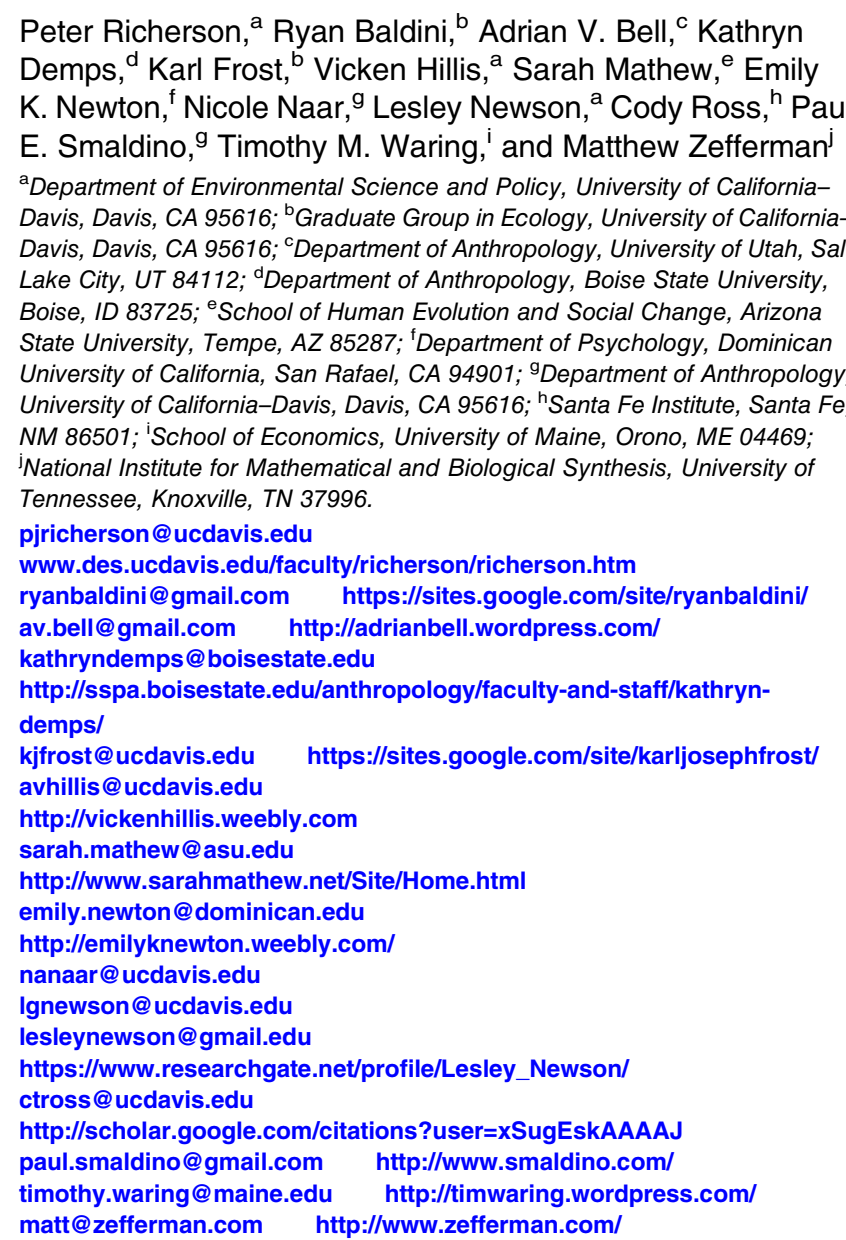

Abstract: The main objective of our target article was to sketch the empirical case for the importance of selection at the level of groups on cultural variation. Such variation is massive in humans, but modest or absent in other species. Group selection processes acting on this variation is a framework for developing explanations of the unusual level of cooperation between non-relatives found in our species. Our case for cultural group selection (CGS) followed Darwin's classic syllogism regarding natural selection: If variation exists at the level of groups, if this variation is heritable, and if it plays a role in the success or failure of competing groups, then selection will operate at the level of groups. We outlined the relevant domains where such evidence can be sought and characterized the main conclusions of work in those domains. Most commentators agree that CGS plays some role in human evolution, although some were considerably more skeptical. Some contributed additional empirical cases. Some raised issues of the scope of CGS explanations versus competing ones.

\section{R1. Introduction}

We thank our commentators for their interesting and thoughtful reflections on our target article. Since commentaries often made more than one point regarding cultural group selection (CGS), in this Response we discuss some of them in more than one section. Our discussion is organized in the following sections, reflecting 13 themes:

Section R2. CGS is a framework.

Section R3. Measuring cultural variation.

Section R4. Little unites the three forms of CGS.

Section R5. Institutions are important.

Section R6. The relationship between psychological processes and cultural evolution.

Section R7. Individual-level processes are important.

Section R8. On what units does CGS operate?

Section R9. Variations in the strength of CGS.

Section R10. Ancient preadaptations.

Section R11. Pleistocene adaptations generated by CGS.

Section R12. Relationship of CGS to the expanded evolutionary synthesis.

Section R13. Selection of extended behavior patterns.

Section R14. The evidence is incomplete.

\section{R2. CGS is a framework}

Commentators Gintis and Mace \& Silva observe that CGS is a framework rather than an explanation. Barclay \& Krupp seem to be expressing the same idea when they describe CGS as a plausible force while arguing that its action needs to be demonstrated in actual cases.

We agree. We see evolutionary theory as a framework, an accounting system, for keeping track of heritable variation through time. A CGS framework is based on the empirical data strongly suggesting that heritable cultural variation often exists between groups at scales larger than groups linked by kinship or a history of reciprocation (target article, sect. 4). Thus, such groups will often respond to some form of selection and many case studies suggest that they do (sects. 5 and 6). The CGS framework has been used to develop explanations for concrete phenomena, for example, the expansion of the Nuer at the expense of the Dinka in the Southern Sudan in the 19th century. Kelly (1985) developed a CGS explanation for the Nuer expansion based on the classic ethnography of Evans-Pritchard (1940). Kelly argued that the bride-price system of the Nuer caused them to count descent more deeply than the Dinka, which made it possible for them to mobilize larger groups of fighters than the Dinka. Hence the Nuer more frequently won fights than the Dinka and incorporated defeated Dinka into their tribes. It is only space constraint and the patience of readers that prevented us from offering many more, and more detailed, examples. The empirical argument in our article is that keeping accounts of group-level cultural variation, and on the impact of cultures selecting genes, is frequently necessary to explain events in human evolution.

Turchin \& Currie also use the concept of a framework to describe CGS. They and colleagues are building a historical database in which they expect that the CGS framework will frequently need to be employed to explain features of human history. Other historically minded scholars have adopted the cultural evolutionary framework exactly because it offers a disciplined method for investigating 
Response/Richerson et al.: Cultural group selection plays an essential role in explaining human cooperation

and comparing quite concrete cases (e.g., Steinmo 2010). It is in the details of such cases that researchers can establish (or not) that an observed cultural difference between groups is actually affecting the outcome of their competition in a manner consistent with an explanation rooted in CGS, as Barclay \& Krupp correctly insist must be done.

\section{R3. Measuring cultural variation}

Three commentaries include remarks on the difficulty of accurately measuring cultural variation: Mace \& Silva, Fuentes \& Kissel, and Ross \& Atkinson. All three critique our use of the $F_{\mathrm{ST}}$ statistic in particular (also see related discussions in sect. R8.) This is an important point. The most ambitious attempt to catalog the world's living cultures was the descriptive work of classical ethnographers. This led to the development of an important database, the Human Relations Area Files (http:/hraf.yale.edu/), that has supported a great deal of quantitative comparative work. The descriptive work on cultural evolution and gene-culture coevolution has been conducted by historians, archaeologists, and geneticists. This is likewise being compiled into databases (target article, sect. 3.2).

Unfortunately, information on variation within societies is very scanty compared to estimates of between-society variation. The individual-level data that we used in section 4.1 to estimate $F_{\mathrm{ST}}$ between various cultural units provide a large number of responses collected from large samples, but we cannot be certain about the extent to which the traits represent culturally heritable variation as opposed to variation influenced by direct environmental effects or genetic variation. We did choose questions (see the appendix at the end of the target article) that seem intuitively to be cultural and related to norms and institutions. It is also undoubtedly the case that competition between the political units known as "countries" is seldom the key level at which selection takes place. Cultural evolutionists have a big task before them to get better estimates of the heritable components of within- and between-group variations.

Despite these limitations, we maintain that our cultural $F_{\text {ST }}$ estimates provide readily available evidence that between-group variation is sufficient for selection to act at the group level (Rogers 1990). Two specific critiques of cultural $F_{\text {ST }}$ require further discussion, however: First, the applicability of the traits measured to CGS, and second, whether variation along geographic clines, not nominal groups, affect the scope of CGS.

Mace \& Silva and Fuentes \& Kissel worry about whether our cultural $F_{\mathrm{ST}}$ measurements assess variation relevant to cooperation. We note that, (1) many of the cultural traits from which we derived cultural $F_{\mathrm{ST}}$ estimates do reflect cooperative altruism, as is made clear in the appendix. For example, we include questions about willingness to sacrifice personal resources for a public good, specifically in the domain of climate change, government involvement in health care, and taxation by the state. Further, (2) we note that there is no reason why we would need to limit cultural $F_{\text {ST }}$ calculations to beliefs or behaviors related to altruism or cooperation. Every cultural or genetic trait can have its own evolutionary trajectory, and this trajectory can be decomposed using the Price equation (Price 1970) into change due to individual-level and group-level effects.
Moreover, altruistic traits are arguably the least interesting traits with which one could study the relative effects of individual-level and group-level selection quantitatively. By definition, the regression of individual-level fitness on individual-level allele frequency is negative for any altruistic trait (a result from Price 1972). The adaptive evolution of an altruistic trait therefore must be due to group-level selection (although not necessarily CGS). Investigation of intergroup partitioning of variance in beliefs related to abortion is interesting in that such beliefs relate very directly to fitness outcomes and could, in principle, be studied quantitatively.

Further, broader cultural differences between groups, or measurements across a range of cultural traits, may approximate the relevant suite of differences resulting in a cultural expansion due to group selection. In this view, our measurements of $F_{\mathrm{ST}}$ are trying to estimate the theoretically important heritable variation that selection might be acting on. Some of that variation is, of course, generated by a complex variety of processes. For example, the military expansion of the Nuer at the expense of the Dinka (Kelly 1985) is presumably not just due to differences in political organization. Differences in other cultural traits, such as subsistence strategy and bride wealth, are likely also important. Likewise, modern political groups differ on a large range of possibly integrated issues that may cause one group to increase and the other to dwindle. The multidimensional quality of cultural expansions often requires us to try to identify and quantify the heritable variation that selection may be acting on. Similar levels of complexity are seen with genetic variation. Genetic $F_{\mathrm{ST}}$ measurements must also concede to complexity. Genetic quantitative characters are influenced by multiple contextual and contingent environmental variables within the processes of, for example, gene regulation, phenotypic plasticity, development, and epigenetics more generally. See our discussion of the problems biologists have linking genes to phenotypes in section R14, which is quite parallel to the difficulties in linking questionnaire responses to behavior.

Ross \& Atkinson raise the issues of nominal groups and spatial variation. Our measure of cultural $F_{\mathrm{ST}}$ is a direct expression of the partitioning of variation in questionnaire responses across the classification of individuals into discrete categories or groups. We have computed cultural $F_{\text {ST }}$ values between ethnolinguistic, political, and religious groups (appendix), adding to other work computing cultural $F_{\mathrm{ST}}$ between a larger sample of nations and four smallscale East-African societies (Bell et al. 2009, Tables S1 and S2). These categories are in some sense ad hoc, in that we could classify these same individuals into any number of other categories/groups and produce a different $F_{\text {ST }}$ value. However, the data we used were obtained from opinion surveys which sampled units that the survey formulators considered to be meaningful social units. We make no ontological assumption that empirical group boundaries are discrete, or that any one particular group classification is true. We simply illustrate that, if we assume a given classification of individuals into groups, we can calculate the scope for group selection to act on the focal cultural trait as a function of this classification. It is true that many human groups have formal criteria for membership and are often symbolically marked by language, dialect, and other differences. Such groups often compete (target article, sect. 5). In section 2.1 we discussed how symbolic 
Response/Richerson et al.: Cultural group selection plays an essential role in explaining human cooperation

marking can create boundaries to the flow of culture between groups. These boundaries are never completely impermeable. The project of understanding just how cultural diffusion is regulated from an evolutionary point of view is in its infancy (Jordan 2015), although a considerable literature exists on how symbolic and formal boundaries affect the diffusion of innovations.

Ross \& Atkinson's finding that geographic variation explains more variation in folktales than currently observed "group boundaries," shows that defining the group structure of a human population is complicated, but we cannot conclude from this that it is a worthless endeavor. Clinal geographic structure is a kind of group structure, as is nominal group identification. A cultural $F_{\mathrm{ST}}$ for a given trait could be calculated using nominal group membership (religion, political party, etc.), or using classification based on geographic structure to any arbitrary level of geographic resolution (nation, state, county, city, postal code, area code, city block, etc.), or even on classification based on the interaction of geographic structure and nominal group membership. The scope of group selection to operate on the given cultural trait could be analyzed in light of each of these classifications. Of course, to understand the system under consideration requires more than estimating the scope for selection. We have to move from the scope for selection to the concrete norms, institutions, and payoffs to variant behaviors to understand what is evolving and why. Folktales may encode norms and be relevant to some CGS process acting on the groups in which they become common, or they could merely spread because individuals find some of them sufficiently entertaining. See also Barclay \& Krupp's comments on the need to understand how a cultural trait affects the success of individuals and/or groups in particular cases.

\section{R4. Little unites the three forms of CGS}

The study of cultural evolution has long been bedeviled by the complexity of the selective processes operating on cultural variation. Natural selection is important; the cultural traits of individuals who survive and reproduce are more likely to be passed on than those of individuals who do not thrive. But the capacity of individuals to decide (consciously or unconsciously) what cultural variants to learn and what to pass on is also important. Individuals also influence the innovation of new cultural variants. Moreover, collective decision-making occurs in many societies using institutions like tribal councils and parliaments. All of these processes act as "forces" influencing the direction of cultural evolution. Cavalli-Sforza and Feldman (1981) called these latter types of forces "cultural selection," and Boyd and Richerson (1985) talked about "decisionmaking forces" arising from individuals choosing how to behave given the choices offered by their culture and the opportunity to innovate. Morin is quite right that one form of CGS we describe, the differential growth, proliferation, and extinction of cultural groups, is a form of natural selection. The other two, differential imitation and differential migration, are selective decision-making processes, not natural selection. The main thing all three have in common is that they are driven by variation among groups. We are not wedded to any particular terminology in this matter. If people feel more comfortable seeing these as three distinctive processes rather than ones with enough family resemblance to merit the CGS term, we have no objection. At any rate, we hope that the similarities and differences are spelled out well enough in section 2.

Morin's more substantive claims are debatable. He argues that natural section on cultural variation is more likely to favor altruism (traits costly at the individual level) than selective imitation and selective migration because the latter are based on individual decisions. There are two problems with this argument. First, imitation and migration decisions are typically made in the face of considerable causal opacity. The decision maker, even if rational and self-interested, cannot understand all of the causal connections between an institution and the benefits it confers. The imitator and especially the migrant make their decisions on the basis of results, not causal pathways. If a measure of altruism is essential to make an institution work, at least some imitators or migrants may acquire the habit of altruism by imitation or teaching. It is good to recall that if a group selection process is favoring altruism, it will be because, averaged over all the altruists in the meta-population, altruistic types do better than non-altruists. In currently highly successful populations, altruists may be relatively numerous, even if not as successful as some of the rarer non-altruists in the successful population. Unless immigrants or imitators can compute the advantages of altruism versus defection rather accurately, they are likely to often imitate the more numerous altruists. Second, recall the tribal social instincts hypothesis (sect. 2.2). Humans are plausibly well-adapted genetically to living in societies in which a measure of altruism is necessary to produce the benefits of cooperation. In public goods game experiments, cooperation gets established if a sufficient number of "leaders" pay costs to discourage selfish behavior, using whatever tools the experimenters give them, such as punishment or communication (e.g., Baum et al. 2012). A minority of people are rationally selfish and a plurality are conditional cooperators, yet in the laboratory with enough tools to work with, altruists can establish near perfect cooperation. This result is common in Western samples, but worldwide behavior is more variable (Herrmann et al. 2008). This finding is consistent with theoretical analyses which show that selection for altruism tends to be frequency dependent (Boyd \& Richerson 1992b). For example, if the costs to altruistic punishers for punishing a given defector are low relative to the cost imposed on the defectors, the frequency of altruistic punishers at equilibrium can be small in proportion. At least in the lab and in theory, a sufficient minority of altruists is necessary to generate cooperation in sizeable groups of non-relatives. There may well be a genetic basis for this altruism driven by culture-led gene-cultural coevolution (sect. 2.2).

Morin's remark that acquisition of behaviors by diffusion will not necessarily lead to demographic consequences is technically true but misleading. Borrowers can acquire a neutral cultural variant that is statistically but not causally related to the success of the donor society. Normally, borrowers expect, or at least hope, that the cultural trait they have copied will have a similar positive effect on the functioning of their society. Once a variant is transferred, it will often have similar effects in the host society as it did in the donor society. However, we should not underestimate the problems of mismatches with other institutions, with 
Response/Richerson et al.: Cultural group selection plays an essential role in explaining human cooperation

other aspects of culture, and with the environment. The decidedly mixed results of efforts to transfer modern Western institutions to the former European colonies after WWII testify to the problem (Gibson et al. 2005). We do not see the relevance of any alliance between the donor and borrowing society. Competing organizations often borrow institutional and military innovations from rivals (see sect. $5.2)$.

\section{R5. Institutions are important}

Several commentaries underscore the importance of institutions. Institutions only operate when a substantial number of people obey their dictates; hence they are inherently group-level traits (sect. 4.3). At the same time, the schedule of rewards offered for conformity to the institution damps down individual-level variation in behavior in the groups that are operating the institution. The punishment of non-conformists prevents them from benefitting from the rewards the institution offers. Institutions vary from group to group. CGS may well act on other sorts of cultural variation, but if institutions exist they are prime candidates to evolve by such processes.

Read argues that CGS does not take into account that cultural idea systems are constituted at an organizational rather than an individual level. Perhaps we were not sufficiently clear, but, in the main, what institutions do is create organizations. Kinship systems as idea systems are used by many peoples to create kin-based organizations such as clans. It is the concrete organizations that actually compete for resources. Ideas may compete directly to be represented in human brains, as in the cross-cultural borrowing we discuss. Presumably, the attractiveness of the institutions of other societies is mostly related to potential adopters' estimates of how much an institution would improve the functioning of their own society. The Netsilik seal-hunting partnership cultural idea system is an institution in our terms, and the Netsilik use it over and over again to create concrete partnerships. Mace \& Silva underscore the important point that kinship norms, political systems, and religion are generally group-level traits.

Houdek \& Novakova introduce the interesting idea of frozen plasticity. Their idea is that as societies become more institutionally complex, interactions between them make it increasingly difficult to change dysfunctional institutions. Eventually, large-scale events like the loss of wars or major natural disasters lead to a punctuated restructuring of social institutions during which time innovations are made, often prosocial ones. Turchin (2006) makes a similar point. We did not intend to imply that the CGS framework is committed to steady and irreversible progress toward better institutions, and if we seem to have done so, we apologize. The stylized facts suggest a trend in the Holocene toward ever more complex societies supporting an ever larger human population, but a more detailed view reveals this global trend to be frequently interrupted by local crises, retrenchment, collapse, and the social extinction of whole societies. A mature theory will have to explain both the trend line and the substantial deviations around it. We might imagine that in crises involving societies with more or less equally complex institutions, the societies with the least frozen and least dysfunctional institutions innovate their way out of the crisis, while their marginally more dysfunctional competitors perish. In this way, on the multi-millennial time scale, the capacity of societies to manage institutional complexity has gradually improved, whereas on the millennial time scale much more complex historical processes dominate the dynamics (e.g., Davies 2011).

\section{R6. The relationship between psychological processes and cultural evolution}

Several commentaries discuss the relationship between psychological processes and CGS, either as alternatives or complements. In our review of the CGS framework (sect. 2 ), we noted the many ways in which the population-level processes of CGS depend upon individual-level psychological processes. In our empirical discussion, we focused on the early-developing cognitive structures that support the human social learning system (sect. 3.1) and the role of norm acquisition in making institutions possible (sect. 4.2).

Henrich \& Boyd's commentary is very close to our view on the relationship between psychological and CGS processes. The important point is that that norms operate at the individual level by reinforcing, co-opting, and modifying gene-based predispositions. For example, Nisbett and Cohen (1996) compared levels of the hormones cortisol and testosterone in Southern and Northern undergraduates (from the U.S.) when exposed to an insult. Southern participants disproportionately subscribed to a culture of honor norm, which on other behavioral measures indicated that Southerners react much more aggressively to mild insults than Northerners. The hormone measures show how emotional centers in the brain stem can be recruited by a culturally transmitted norm (Panksepp \& Biven 2012). Nisbett and Cohen review the historical data related to the evolution of the Southern Culture of Honor and the child-rearing practices that cause it to develop.

When considering gene-based predispositions, it must be remembered that by the end of infancy humans have developed a sophisticated capacity for social learning (sect. 3.1). The genetic and cultural contributions to adult cognitive structures will therefore co-develop from early childhood as well as coevolve in evolutionary time. We as yet do not know in much mechanistic detail how cognition develops or how it evolved. Features like incest avoidance, kin recognition, and basic emotions are probably widely shared with other species, making animal models relevant to the discussion. Historic information extracted from the genome, together with improvements in the conventional paleoanthropological record, may one day tell us much more about how human brains and cognition evolved (Richerson et al. 2010). Cross-cultural developmental studies, which Henrich \& Boyd and their co-workers are pioneering, will help tease apart genetic and cultural contributions to development. We think we know enough to be confident that the outline of the relationship between genes and culture in development that Henrich \& Boyd outline is correct but our current understanding resolves few details.

We are sympathetic to Tooby \& Cosmides' argument that much is built on gene-based social psychological innovations that evolved during the Pleistocene. We briefly reviewed the "tribal social instincts" hypothesis in section 2.2 and discuss it in relation to the sufficiency of individual- 
Response/Richerson et al.: Cultural group selection plays an essential role in explaining human cooperation

level processes in sections R7 here and 2.2 in the target article. Tooby \& Cosmides argue that individuals with neural adaptations evolved in Pleistocene forager societies can self-organize into large cooperative structures without the aid of cultural evolutionary processes. They suggest that ancestral foraging societies were small enough that individual-level social skills are sufficient to explain their social structure, and that these skills likewise suffice to explain the much larger scale and more complex societies of today.

Tooby \& Cosmides question the realism of experimental games that find altruistic or other prosocial behavior. All experiments involve simplifications. It is not clear that the experimental game studies they cite in support of their argument are any more realistic. See Zefferman's (2014a) critique of a simulation study by Delton et al. (2011) in this regard (see also Delton \& Krasnow 2014; Zefferman $2014 \mathrm{~b}$ ). The most ambitious attempts to relate experimental games to the real world are the cross-cultural studies of Henrich et al. (2004; 2010a) (see sects. 6.2, 6.3, and 4.3 in the target article). These studies were conducted mostly in institutionally simple hunting and gathering, herding, and horticultural societies by ethnographers of the people studied. This design was adopted so as to be able to infer from the extensive ethnographic knowledge whether or not game behavior matched behavior in the society concerned. They summarize their main findings as follows:

We draw two lessons from the experimental results: first, there is no society in which experimental behavior is even roughly consistent with the canonical model of purely self-interested actors; second, there is much more variation between groups than has previously been reported, and this variation correlates with differences in patterns of interaction found in everyday life. (Henrich et al. 2004, p. 5, emphasis ours)

In section 6.2 we also briefly discussed the social psychologist Daniel Batson's (2011) experimental program designed to test whether psychological altruism was necessary to explain participants' behavior in circumstances where they could volunteer to aid someone in need. Batson's experiments used deception to create scenarios that were as realistic as possible, and he found evidence that some participants behaved altruistically. Tooby \& Cosmides' discussions of List (2007) and Sheskin et al. (2014) are incomplete. In List's taking treatments, dictator giving was substantially reduced relative to the standard dictator game, but the amount of taking was quite modest. In a treatment where dictators and their partners had to work to earn their money, very few dictators chose to take at all. List emphasizes that prosocial morality plays a big role in his results. In Sheskin et al.'s study, young children, but not older ones, chose to pay a cost to reduce the welfare of others relative to their own. Whether this change with age was a product of an innate developmental process or socialization, or both, is unanswered by this study.

We agree that ancestral forager societies were small compared to the giant societies in which many humans lived during the last 5,000 years, but we question the suggestion that they were so small that individual-level social skills can explain their social structure. Human forager societies are already large compared to chimpanzee and bonobo communities. Forager societies have culturally variable norms and institutions regulating family life and interfamily cooperation. As Hill et al. (2014) show, the band scale units of forager tribes are fluid, and cooperation with many distantly related or unrelated people is common. In ethnographic cases, and by at least the Upper Paleolithic, trade networks linked local groups on a subcontinental scale. It is true that these institutions can be scaled up considerably with a more productive economy than hunting and gathering. The Turkana, in our example in sect. 5.1, use a subsistence economy based on livestock raising and trade to support a society numbering around one million with institutions hardly different from those of most forager societies. It is the largest society known to us that remains without formal leadership institutions, although people vary greatly in prestige. Most societies larger than a few thousand people evolve formal institutions of leadership and governance. We argue that culture-led gene-culture coevolution had extensively remodeled our social psychology some time before 50,000 years ago. We agree with Tooby $\&$ Cosmides that major new genetic adaptations supporting the formation of giant societies are unlikely to have occurred during the last 5,000 years (although minor adjustments may have). It seems likely that large-scale societies arose through a process of institutional innovation, which we understand in some detail in particular cases (e.g., Currie et al. 2010). In section 6, we pointed to evidence that language, social control, and religion and ritual all show evidence of "design" features consistent with the operation of CGS.

Tooby \& Cosmides and Krasnow \& Delton seem to suggest that the study of cultural evolution should be postponed until research in evolutionary psychology has revealed whether or not the patterns observed can be entirely explained by evolved cognitive mechanisms. But that is not how science works. Darwin's explanation of evolution based on heritable variation was valuable even though his theory of inheritance was wrong. Even today our understanding of the genetic inheritance system is incomplete. Krasnow \& Delton contrast the "detailed, unique, and novel predictions" of Evolutionary Psychology ${ }^{1}$ with the "retrodictions" of CGS of facts we already know, such as why humans have languages. Privileging prediction over retrodiction in in this way leads to some odd conclusions. Would they disparage Darwin's work on the Origin of Species because he was merely retrodicting facts he already knew - that species existed and are generally well adapted to their environments? Science often makes good progress when some components of the phenomenon being studied have to be treated like black boxes. Such investigations motivate and guide the science that aims to open up the boxes.

We are as enthusiastic about the adaptationist approach to human behavior as Krasnow \& Delton. Boyd and Richerson's (1985) book is basically an extended evolutionary analysis of how human culture functions as an adaptation. To do this analysis properly, you have to take into account the population-level properties of culture, just as you have to take the population-level properties of genes into account to understand organic evolution. The theoretical work outlined in sect. 2 suggests that one peculiarity of the cultural inheritance system is that it can support considerable variation at the level of groups, and accordingly that it can support the evolution of group functions if there is competition between groups. The target article is mostly an empirical sketch of the evidence available on these axiomatic points. Krasnow \& Delton do not impugn this 
Response/Richerson et al.: Cultural group selection plays an essential role in explaining human cooperation

evidence. Their objections depend upon an argument based on G. C. Williams' (1966) gene-centered view of evolution, a view that he subsequently modified (Williams 1992) to include the idea of multi-level selection, based on the work of Price $(1970 ; 1972)$. Maynard Smith and Szathmáry (1995) attributed the major transitions of evolution (including human origins) to rare innovations that led to the operation of selection at higher levels. Regarding parsimony, recall Einstein's dictum "Everything should be made as simple as possible, but no simpler." Trying to understand human evolution without taking into account the population-level properties of culture is to use too simple a framework.

The field of Evolutionary Psychology, pioneered by Tooby and Cosmides (1989; 1992), has received much criticism for the narrowness of its program (e.g., Bolhuis et al. 2011), but their enterprise fits well into a larger evolutionary science of human behavior along the lines of Henrich \& Boyd's commentary. Barrett's (2015) recent book, and work such as Gervais et al. (2011), show how naturally psychological and population based approaches fit together. In this regard Buss' (2014) textbook Evolutionary Psychology, cited by Krasnow \& Delton, is interesting in that it does not contain a discussion of the human social learning system that has been well described by developmental and comparative psychologists (sect. 3.1). This is an odd omission, given that the human social learning system is perhaps the best-described human cognitive module known to have evolved considerably during the Pleistocene. There is also no discussion of institutions or norms in Buss (2014).

We appreciate Suárez \& Koenig's raising the evidence on the selectivity of even young children's social learning. The broader theory of cultural evolution (e.g., Boyd \& Richerson 1985; Cavalli-Sforza \& Feldman 1981) developed mathematical models to investigate the effect on social learning selectivity based on genetic predispositions and social learning selectivity based on earlier acquired culture. These early models of bias were derived from the literature on persuasion and the acquisition of innovations by adults. The more recent studies of social learning in children have filled important gaps. The idea that children are evaluating potential role models for expertise, truthfulness, conformity to norms, and affiliation with the child's group are important planks in the Tribal Social Instincts hypothesis, showing that children do favor prosocial and in-group models. This, we suggest, reflects an evolutionary history of living in norm- and institution-bound societies, which create a tendency for norms and institutions to culturally evolve in prosocial directions. We are also interested in the variation in children's social learning biases. In section R4 we noted that young adults vary substantially in their strategies in the Public Goods Game. When these young adults were younger, would they have had different social learning biases that led them to acquire a different set of moral norms?

This is consistent with the idea that selection of prosocial tendencies under CGS will be frequency dependent. The rational component of children's imitation may tend to smooth out intergroup differences. However, it is important to remember the observation of Harris (2012) that in many matters children have so little knowledge that they must accept the testimony of adults. In the case of complex artifacts and institutions, even adult experts do not seem to have deep causal knowledge of how these cultural constructs function (Sterelny 2012). Such causal opacity will remove much of the influence of rational choices, and causal clarity will make such choices very powerful. In laboratory experiments with young adults, payoffbiased social learning strategies are commonly used if sufficient information is available, and more conformity is used when information is less definitive (McElreath et al. 2008).

Vigil \& Kruger's suggestion that honest communication of pain is an important part of social life is plausible. It parallels the argument we made for the case of language in section 6.1. More generally, it is common for people to signal their emotional states, positive as well as negative. Signaling systems in which self-interested deception is possible can evolve only if there is a certain level of trust between senders and receivers of signals, especially if the signals are inexpensive. Expressions of pain often trigger helping behavior in others. This is a reliable reaction. It has led to the development of the strategy of terrorist bombers to set off a second bomb as people rush in to aid the victims of the first. Images of the pain suffered by a particular group are used to recruit charitable donations and volunteers to aid them in natural disasters and wars. Vigil \& Kruger provide evidence that people who identify with an in-group can be powerfully affected by images of harm done to in-group members. At the same time, some people exaggerate their suffering to attract aid they do not deserve, and such behavior is disparaged and policed much like any other lie.

\section{R7. Individual-level processes are important}

Several commentaries remind us that cultural evolution includes many phenomena that are distantly, if at all, connected to group-level processes. Most cultural evolutionary analysis asks how individual-level decisions affect the evolution of culture where the agents making the decisions are assumed to be boundedly rational individuals pursuing their inclusive fitness interest (e.g., Boyd \& Richerson 1985). In this regard we endorse many of the remarks in the commentaries of Amir, Jordan, \& Rand (Amir et al.); Singh, Glowacki, \& Wrangham (Singh et al.); Tooby \& Cosmides; Houdek \& Novakova; and Nesse. However, when it comes to human social life, we are convinced that individual-level processes are often heavily influenced by the products of CGS processes.

Amir et al. present an excellent model of individual decision-making that we think is largely compatible with CGS. In their social heuristics hypothesis (SHH), Amir et al. argue that individuals internalize the strategies that are typically advantageous in daily life. We argue that institutions substantially determine which strategies are typically advantageous. Institutions are group-level traits that evolve due to CGS (sect. 4.3). Amir et al. give the example of how voting institutions might guide individual behavior. Importantly, voting institutions, and social choice institutions more generally, vary widely across groups, even across modern democratic states. While individual behavior might be explained by SHH, given a particular set of voting institutions, voting institutions themselves are group-level traits because an individual cannot unilaterally decide to use different voting rules than the rest of her group. Since voting institutions can only change at the group 
Response/Richerson et al.: Cultural group selection plays an essential role in explaining human cooperation

level, they are subject to CGS. If, as Amir et al. suggest, individuals can migrate to join groups with voting institutions more to their liking, this would be a mechanism by which CGS could operate (sect. 2.1). Additionally, Amir et al.'s idea that institutions homogenize individual behavior is precisely what potentiates CGS processes by suppressing individual-level variation and creating group-level variation. Because sanctions and rewards can stabilize institutions that have highly variable functionality, institutional innovators cannot easily move from suboptimal to optimal institutions (see sect. 4.3). In summary, we see SHH as a theory to explain human behavior, given variation in norms, institutions, and innate predispositions, whereas CGS and gene-culture coevolution aim to explain norms, institutions, and innate predispositions themselves. An important future project might be formally integrating ideas from $\mathrm{SHH}$ and CGS to more tightly link gene-culture coevolutionary models with the findings of cross-cultural experimental psychology and economics.

Both Singh et al., and Houdek \& Novakova make claims about how endogenous processes of cultural change will affect the emergence of group-beneficial institutions. Singh et al. argue that endogenous processes of cultural change can lead to norms that are group beneficial because the interest of powerful leaders align with the interest of the group. They therefore argue that CGS is unnecessary for explaining group-beneficial institutions. In contrast, Houdek \& Novakova point to the several pathways by which the action of self-interested actors can wreck the success of a group. We agree that punishment can sustain antisocial behavior as readily as it can sustain prosocial behavior and know that history is rife with examples of coercive, extractive institutions that were not optimal for the group, but benefited the people imposing these rules (e.g., Turchin \& Nefedov 2009). And we argue that this is exactly why the processes of CGS are needed to explain the unusual level of prosocial outcomes that we do see in human societies, such as property rights, redistributive mechanisms, safety nets for those struck by misfortunes, military defense, and public capital facilities.

We think the diversity of opinion on this issue reflects the reality that both self-interest and cooperation do occur. Self-interested actors maneuver rules to their advantage, as evidenced by the corruption that plagues many institutions. Furthermore, individuals with more bargaining power in a situation - people with wealth, influence, coercive authority, weapons, private militias, family ties, social connections, and so forth - will try to extract more for themselves. Such a situation often leads to rules and outcomes that are not beneficial for the group. In fact, it is seldom (if ever) the case that rules and outcomes that are optimal for the group are achieved.

If self-interested leaders often create rules that also happen to benefit the group, as Singh et al. argue, shouldn't group-beneficial outcomes be widespread in animals? Hierarchies and power differences are extremely common in social animals. Yet, the presence of powerful individuals with coercive capacity has not created animal societies with complex rules for solving public goods problems, or enforcing property rights, or any of the myriad other forms of cooperation commonplace in human societies. When cooperative outcomes are observed in animal societies, they are typically among kin. Singh et al. do not consider the full strategy space of rules that are available to self-interested leaders. They bring up the example of Cheyenne leaders, who implemented a rule regarding thieving of horses: "Now we shall make a new rule. There shall be no more borrowing of horses without asking... [If] the taker tries to keep them, we will give him a whipping." They conclude that the leaders impose such a rule because preventing theft and maintaining social order benefits the leaders. But wouldn't a rule in which the leader takes this stolen horse for himself benefit the leader even more? What about a rule requiring such thieves to hand over their wives to the leader? Or a rule that says that the punishment only applies to people the leader does not like? Let us assume for a moment that leaders do always attempt to establish rules in their own interest. Nevertheless, they will occasionally make rules favoring group interests either because of an alignment of elite and non-elite preferences, or because of errors of judgment or exogenous changes. No matter the intent of the original rule-makers, CGS processes will act to favor such rules and disfavor rules that are actually selfish. We do not want to say that the interests of leaders and followers never align without evolved institutional incentives, just that this mechanism alone can only account for the limited amounts cooperation found in societies with dominance hierarchies.

Tooby \& Cosmides speculate that modern institutions can be far better explained by neural adaptations in dense persisting social networks of intelligent cultural agents than by CGS. Once again, one cannot discuss individual behavior without considering the possibility that humans have adapted by gene-culture coevolution to live in societies shaped by CGS. As we discussed in section R4, it takes a largish minority of altruistic punishers to establish cooperation in laboratory public goods games, consistent with CGS models (Boyd et al. 2003). In societies with poor institutions, experimental participants can't establish cooperation using the punishment tool (Herrmann et al. 2008). Leaders are leaders either by virtue of coercive power or by grants of prestige from their followers, or both. Prestige is a strong motivator of behavior (Henrich \& GilWhite 2001), and even the powerful may act in the group interest to gain prestige. Also, selfish political operators can't advocate for new institutions based on selfish advantage unless they are dictators. To the extent that they need the consent or at least the acquiescence of others, they will at least need to convince followers that the innovation is in the group's interest even if the group is only a narrow ruling class. If the new institution turns out to be only in the leaders' interests, their prestige and perhaps authority will often by diminished by unpopularity, resistance, and revolt (Insko et al. 1983). Thus, endogenous processes can aid in the creation of group-beneficial institutions, but for this to work on any scale, the prosocial instincts would have had to be favored by some process like CGS acting via gene-culture coevolution in the past.

Nesse brings up the very important concept of social selection. Certainly many non-human social phenomena are shaped by social selection. Indeed, it seems likely that in any social species social selection plays an important role. For example, the mutual grooming seen in many social mammals must be the product of social selection disfavoring individuals who were unacceptably rough or ticklish. The very ubiquity of social selection argues against it being capable, on its own, of generating societies of the size and complexity of human societies. Runaway sexual 
Response/Richerson et al.: Cultural group selection plays an essential role in explaining human cooperation

selection is a common phenomenon. If a runaway form of partner choice social selection were possible, shouldn't we see many non-human societies with extensive cooperation between non-relatives? As we argued in sections 2, 4.3, and 6.2 of the target article, culturally transmitted institutions act to greatly amplify social section. They are rules that deliver rewards and sanctions to individuals, they vary substantially from society to society, and they influence the outcome of inter-organizational competition. We think it is CGS on institutions that put humans on a runaway path to semi-domestication. Pleistocene culture-driven geneculture coevolution would have favored the evolution of guilt, pity, commitment, empathy, and generosity, much as Darwin (1874) imagined in the epigraph from The Descent of Man at the start of our target article. As we elaborate in sections R10 here and 4.3 in the target article, even the ubiquitous simple partnerships, like marriage and trade, are heavily institutionalized even in simple societies.

Lamba describes a field study of variation in cooperation (measured by an experimental public goods game) in a sample of 14 villages within an ethnic group in India (Lamba \& Mace 2011). Some of this variation could be explained statistically by population size, age, and sex, which Lamba attributes causally to individual-level effects. As far as we can see, it could also involve some component of micro-cultural variation, for example, norms of cooperation might differ by gender. However, all experimental game studies we know of find ample individual-level variation, including the studies of Henrich et al. (2004; 2006; 2010a). Henrich et al. (2012a) report that the variation between communities, estimated for some but not all of their samples of societies, is approximately the same as the variation that Lamba and Mace (2011) reported and is rather smaller than the between-cultures variation in the Henrich et al. studies. We do not doubt that as the scale of measurement of variation shrinks, individual-level effects will become increasingly important, all the more so to the extent that cultural variation is concentrated at a larger scale. If people in a regional-scale society all operate under the same institutions, perhaps the main sources of individual variation in behavior are environmental and genetic. Lamba (2014) also reports finding only modest effects of cultural transmission as measured by changes in individuals' behavior between two rounds of a one-shot public goods game. In the second round, some individuals tended to conform to the mean contribution in the first round and some tended to adopt the payoffmaximizing contribution, but there was a strong mode at no change. This finding is in line with laboratory studies of social learning (e.g., McElreath et al. 2008). A major limitation of such studies is that one brief social learning experience cannot be expected to have a large impact on habits and norms that have been acquired over a lifetime. The experiments reported in section 4.2 of the target article show that children acquire norms quite readily.

\section{R8. On what units does CGS operate?}

Some authors raise important issues concerning the units and levels of selection. As we remarked in section 1, the CGS framework can be applied to many different concrete entities; it includes the concept of multi-level selection. It is applicable to easily copied fragments of culture (which
Whiten \& Erdal call “meme-complexes"); to structural elements within a population, such as business organizations (discussed in sect. 5.2); to ethnolinguistic groups, corporate kin groups, clubs, nations, religions; and even to multiethnic empires (Turchin 2009).

Whiten \& Erdal suggest that the relevant groups for CGS should be groups of cultural replicators (meme-complexes), rather than groups of people. While meme-complexes, like other cultural traits, can vary between groups or increase and decrease in frequency as part of a CGS process, in the CGS framework, they are not the groups themselves. However, if meme-complexes are self-reinforcing, they may become particularly stable within groups once adopted, similar to Smaldino's (2014) concept of a "group level trait." Groups may, as a result, become strongly influenced by particular meme-complexes, and cultural group selection may operate on meme-complexes with particular force.

When applying the CGS framework to a particular case, we think it is as important to analyze the ecology of competition and cooperation when defining groups as it is to analyze the patterns of cultural variation. CGS is likely to be operating where there is a coincidence of a pattern of cultural variation and a pattern of conflict. Lamba seems to suggest that ethnolinguistic groups have a privileged position in the CGS framework. That is not our view. She finds considerable variation in cooperative behavior within the ethnic group she studied, partly related to demographic and ecological processes. This is not surprising once one takes into account the relevant scales of collective action for the traits she examines. For example, Lamba describes an experiment where villagers are asked to divide a measure of salt between themselves and other villagers. If salt division is a collective action problem typically encountered at the scale of villages, then villages would be the relevant group in the CGS framework. The ethnolinguistic group would not be the relevant group unless the problem of salt division could be solved by collective action at that larger scale. Similarly, one of the best studied possible examples of CGS is warfare between clans within ethnic groups in Highland New Guinea (Soltis et al. 1995). Mace \& Silva and Acedo-Carmona \& Gomila also describe field situations in which the locus of cooperation and conflict is at smaller scales than what a Western anthropologist might define as a cultural group. In section 5.1 we described the cooperation and competition among the Turkana which are organized at the level of a large ethnic group. In section 5.2 we discussed business organizations that are typically subnational in scale, although large modern firms are often multinational in their operations and multi-ethnic in their staff. Clearly, human societies are both complex and diverse. Doing full justice to this diversity is beyond the scope of our target article, but we would not want to be misunderstood as thinking that the CGS approach is committed to a particular claim about the kinds of groups that compete.

Acedo-Carmona \& Gomila raise the important issue of the persistence of medium-scale diversity within state-level societies. They argue that CGS predicts regional cultural homogenization in the long run, and that such homogenization does not occur in their cases in Oaxaca and Northern Ghana. This is not quite right because, while it is true that, in a stable environment, a simple CGS model predicts that competing groups will have similar group success at equilibrium, social evolution is a complex design problem 
Response/Richerson et al.: Cultural group selection plays an essential role in explaining human cooperation

(Boyd \& Richerson 1992a). There are likely to be many equally successful but rather different ways of organizing societies. Furthermore, medium-sized organizations often evolve better institutions from the bottom up than can be created by top-down organizations like nation-states (Ostrom 2005). CGS for cooperative institutions at the scale of neighborhoods, villages, churches, business organizations, and the like probably often favors institutions that protect the local autonomy of medium-sized organizations which are successful at solving a particular problem. For example, around Lake Titicaca, Peruvian lakeside villagers actively defend fishing territories that are completely illegal under Peruvian law. Yet they succeed in defending these rights against trespass by neighboring villagers, interference from state officials, and use by state-sanctioned private companies (Levieil \& Orlove 1990). Multiple equilibria in complex systems and relative efficiency of mediumscale organization will therefore counter the homogenizing tendency of CGS and preserve medium-scale diversity within bigger units like states or ethnolinguistic groups.

Santana, Patel, Chang, \& Weisberg (Santana et al.) make a similar argument focused on the phenomenon of religious syncretism. They point out that selective conquest, migration, or imitation seldom results in total replacement of pre-existing culture. More often there is a new hybrid culture, a process sometimes called "ethnogenesis." Among other things, ethnogenesis offers the possibility of cultural recombination, discovering favorable new combinations of cultural traits not present in either of the contributing lineages. As Santana et al. correctly observe, there must be evidence that traits acquired or retained during ethnogenesis actually had group functional effects that drove acquisition or retention if we are to attribute their evolution to CGS. We pointed to literatures in section 5 that document how competing organizations use techniques such as head-hunting and espionage in highly organized ways to acquire the successful techniques of rivals. (For the borrowing of military technology, see Horowitz [2010].) Still, Santana et al. are surely correct that many cultural traits spread between groups or are retained in ethnogenesis for their individual-level functions, not to mention the frequent diffusion of harmful practices such as tobacco smoking and the consumption of fast food. The quantitative study they suggest to examine the levels of organization that are affected by a sample of diffusing innovations would be very interesting.

Santana et al.'s case of the Catholic veneer adopted by local Mexican communities is an interesting example related to those described by Acedo-Carmona \& Gomila. As Foster's (1960) classic account of America's Spanish heritage documented, the pattern of highly heterodox local beliefs in America replicates a pattern found in Spain, despite energetic and largely successful attempts by colonial authorities to prevent the spread of unorthodox religious ideas from Spain to the New World. Again, local differentiation of beliefs could be related to multi-level CGS favoring one set of institutions at the village scale correlated with local heterodox religious beliefs and another associated with Catholic orthodoxy among national elites. As we discuss in section 6.3, religions seem to function to provide symbolic group boundaries, moral justifications for institutions, and rituals that express group solidarity. The "veneer" inter-level relationship between elite Catholic orthodoxy and village-level heterodoxy is particularly well studied in the case of the New Mexican Pueblos. The Pueblo peoples resisted Spanish domination violently, culminating in a spectacularly, if temporarily, successful revolt in 1680. The Spanish returned 12 years later much chastened. "Veneer" Catholicism symbolized the Puebloans' acquiescence to Spanish overlordship in exchange for a Spanish tolerance of substantial Pueblo autonomy, an arrangement that persisted under Mexican and U.S. rule (Simmons 1979).

Regarding Mace \& Silva's Northern Ireland case study (Silva \& Mace 2014), we appreciate their raising the issue of parochial altruism. Some plausible CGS models do predict the evolution of a combination of in-group cooperation and out-group hate, but in section 4.2 we dissociated ourselves from the view that this relationship is a necessary product of CGS in general. Empirically, no law-like association between in-group cooperation and out-group dislike has been found (Brewer 2007). This is not hard to understand. Peaceful relations between groups make possible gains from trade while warring with neighbors is generally negative sum, costly to both sides. Only in very asymmetric conflicts or surprise attacks are aggressors reasonably certain to come out ahead and in the case of surprise attacks, victory is usually temporary. Large organizations, like states and empires, often offer mediation or legal services to settle disputes between the smaller organizations they contain. When such services are efficient and just, the constituent organizations are typically happy with the results (North et al. 2009). In simple societies, the lack of mediation institutions can lead a society toward extinction, at least in a competitive environment (Knauft 1985). Even in cases where enmities are raw and deep-seated, as in Northern Ireland, mediation by the United Kingdom and Ireland, assisted by the United States, eventually damped down violent conflict there.

De Dreu \& Balliet's point that intergroup interactions are mixed motive rather than zero sum is likewise appreciated. Groups that trade and make peace may also be competitors. Thus, firms in an industry may come together to form a trade group that lobbies, advertises, and does research to benefit the industry as a whole, while still competing vigorously on price, quality, and so forth. In our example of the 19th century dye industry in section 5.2, it was cooperation between competing German firms to lobby states to create research professorships in chemistry that gave German firms collectively a decisive advantage over their British rivals. Societies find it expedient to ally with others to fight a threatening common enemy even if they continue to compete vigorously in other spheres. Any organization that does not have a robust set of institutions to make possible trade and alliance is liable to be outcompeted by ones that do. The Comanche dominated the Southern Plains of North America militarily for a couple of centuries in part because they made alliances and trade relations with other Indian tribes and with Europeans (Hämäläinen 2008). A tight coupling between in-group love and out-group hate would make alliance-forming difficult, something that CGS is likely to disfavor, all else equal. Models also exist which show cooperation arising in a game to survive in harsh unstable environments, even in the absence of conflict between groups (Smaldino et al. 2013). A failure to cooperate may lead to group extinction due to a failure to extract sufficient resources to maintain a viable population, even in the absence of competition. We do not classify this as a form of CGS because it does not 
Response/Richerson et al.: Cultural group selection plays an essential role in explaining human cooperation

involve between-group variation and inter-group competition, but that does not mean it is unimportant in favoring group-level cooperation. This process would also tend to produce in-group love but not out-group hate.

Herzog proposes that pet-keeping can best be explained by CGS. He presents no obvious group fitness benefits of pet-keeping. The best evidence provided suggests only that, as the author states, pet-keeping is like fashion, and it spreads through cultural transmission. It is influenced by parental preferences, prestige, and conformity. Petkeeping is apparently not a cultural trait that is appreciably influenced by CGS processes. The fact that people readily form attachments to pets illustrates how flexible the human attachment system is, but the capacity to form attachments to social groups is a much more consequential example for CGS processes.

\section{R9. Variations in the strength of CGS}

Commentators Gelfand, Roos, Nau, Harrington, Mu, \& Jackson's (Gelfand et al.'s) point that the strength of CGS processes will vary as a function of the threats a society faces makes sense. Some of the changes in the commitment to groups are short term in nature, for example, the compulsory mobilization of a society fighting an existential war. The Mu et al. (2015) study shows that priming subjects to think of an out-group threat immediately increases cooperation. These are the sorts of psychological predispositions that might result from a long evolutionary history of group living (sect. 2.2). Boehm's (1984) study of Montenegrin feuding described institutions for suspending feuds when it was necessary to raise a tribal army to fend off Muslim invaders. Montenegrins' willingness to suspend feuds was no doubt aided by the clear danger to the whole society posed by potential Muslim conquest. Other changes are longer term, as evidenced by institutional differences across American states. Some scholars have argued that these differences have a historical character derived from patterns of colonial immigration from different parts of Britain (Fischer 1989; Nisbett \& Cohen 1996). Immigrants may also settle in regions and situations to which their norms and institutions are adapted, making the correlations in the $\mathrm{Mu}$ et al. study hard to interpret causally, as Gelfand et al. recognize. The role of possible genetic adaptations to living in tight versus loose societies is interesting, but the arrow of causation also has to be established. Human genetic diversity is strongly correlated with distance from Africa, implying that drift was a strong factor in our evolution (Pickrell \& Reich 2014). Institutions might coevolve with gene frequency differences that were a product of chance factors. Ancient DNA can be used to characterize the genotypes of people before and during a putative culture-caused selective sweep and hence rule out the possibility that a pre-existing genetic difference influenced cultural evolution (Itan et al. 2009).

\section{R10. Ancient preadaptations}

It goes without saying that human culture and CGS processes rest on ancient roots. Even something as novel as our capacity to form large-scale societies is likely to be a mere modification of traits present in our ancestors.
Chisholm, Coall, \& Atkinson (Chisholm et al.) offer mother-infant attachment as an example. This is a bond that is practically required by mammals and is thus phylogenetically ancient. It is one that has been remodeled for many different purposes in the course of social evolution, just as Chisholm et al. argue. See also Panksepp and Biven (2012, Chapters 8-10). Attachment to groups, for example, looks as if it depends upon some of the same subcortical roots as individual-individual attachments (Haslam 2001).

Burkart \& van Schaik argue that cooperative breeding provides a much more recent precursor of why humans rather than some other great ape evolved into the hyper-cooperative niche. The comparative primatology of cooperative breeding is certainly striking, as their experiments show. Both generalized cooperation and social learning appear to be enhanced in cooperative breeders. Cooperative breeding is certainly a candidate to have favored more cooperation in early hominins and to have amplified the advantages of social learning by exposing selective learners to a greater number of potentially useful cultural variants. Larger social groups could also have favored improved communication. Our only worry is our original one in the target article (sect. 1) that the paleoanthropological record is almost silent on the evolution of social organization. Stiner and Kuhn (2009) argue from diet and artifact types that a sexual division of labor did not evolve until the Upper Paleolithic, suggesting that large-brained hominins, closely related to modern humans, had very different social organization.

Human cooperative breeding is heavily institutionalized in ethnographically known small-scale societies by rules about kinship and marriage. Mathew et al. (2013) suggest that the human capacity to cooperate in many different activities is based on our ability to create institutions to manage diverse forms of cooperation (see sect. 4.3 of the target article). Thus, an alternative hypothesis is that simple institutions preceded cooperative breeding in humans and made it possible. Perhaps families were one of the earliest social institutions. On present evidence both hypotheses seem equally plausible, not to mention any number of more complex coevolutionary scenarios one can imagine in which genes and culture played alternating leading and lagging roles.

\section{R11. Pleistocene adaptations generated by CGS}

Commentators Gintis, Fuentes \& Kissel, and Whiten \& Erdal observe that the target article includes little mention of CGS and culture-driven gene-culture coevolution in the Pleistocene. They outline several examples of adaptations that may very well have been built by these processes far earlier than the examples we discuss. We agree that these are good candidates to be explained by CGS and culturedriven gene-culture coevolution along the lines of the tribal social instincts hypothesis (sect. 2.2). However, in this article we emphasized empirical evidence that is as close to unassailable as it is possible to get. Inevitably, this resulted in an emphasis on more recent cases. Humans seem to have been quite rare before about 50,000 years ago (Li \& Durbin 2011). Hence, the paleoanthropological data record is scanty and hard to interpret. Language, institutions, and other traits that are central to the operation of CGS leave poor proxies in that record. The temporal resolution of the record is poor, so it is hard to be sure whether 
Response/Richerson et al.: Cultural group selection plays an essential role in explaining human cooperation

cultural evolution is the leading or lagging variable in coevolutionary events. Ongoing improvements in the paleoanthropological record will help make hypotheses about deep time events more testable, and there is great promise for extracting more genetic history from living autosomal DNA, supplemented by recovery of subfossil DNA (Richerson et al. 2010). Gintis, Fuentes \& Kissel, and Whiten \& Erdal raise questions about the most important events in our evolutionary history. We look forward to advances that will help us better understand them.

\section{R12. Relationship of CGS to the expanded evolutionary synthesis}

We thank Fuentes \& Kissel for raising the issue of the relationship between CGS and the extended evolutionary synthesis (EES). We are quite willing to include CGS as part of the EES. Cultural evolutionists often cite and are cited by other EES authors. For example, we cited Laland et al. $(2010 ; 2011)$ in our target article, and both of these papers cite numerous publications by other cultural evolutionists. If our tribal social instincts hypothesis is correct, the evolution of the cultural inheritance system under CGS had major impacts on the genetic component of our social psychology, not to mention many other aspects of our phenotype. Two of the three CGS mechanisms we discuss are based on selective decisions, not natural selection, and as we have remarked here in section R4, individual and collective decisions regarding institutional innovation and adoption are potentially biased in a prosocial direction due to the ancient remodeling of our social psychology by CGS. In section 6.2, we discussed the genetic evolutionary effect that punishment on deviants would have if indeed there is (or was) genetic variation in tendencies to deviate from the strictures of norms and institutions. Barclay \& Krupp defend a decidedly nonEES picture in their remark that proponents of CGS are confusing proximate and ultimate causation. Exactly who is confused is the central issue in the debate over EES. For those who accept the evidence for culture-led geneculture coevolution, it is the opponents of the CGS who are confused (Laland et al. 2011).

\section{R13. Selection of extended behavior patterns}

Simon makes a useful point in her commentary that selection on patterns of behavior has to consider the effects on fitness that accrue over time and across individuals. Individuals may pay a cost for being prosocial at one time but be rewarded later because they had achieved a reputation for generosity. Carriers of a cultural variant or genotype encouraging generosity may incur a net lifetime cost not paid by the ungenerous. In the case of such an altruistic groupselected trait, generosity may increase because even though it is counter-selected within every group where it is present, the success of groups with many generous types relative to those with few can result in the generous trait increasing in frequency in the meta-population. Thus, Simon is not quite correct to say that "cooperation ... was selected because it commonly enhanced the individual's long-term fitness" in the sense that there is, by definition, always a withingroup disadvantage to altruistic generosity.
Much of evolutionary theory is devoted to understanding how selection trades off costs and benefits of extended patterns of behavior. Life history theory studies how the effect of sacrifice of reproduction at one stage of the life history can or cannot be favored by increased reproduction at another stage. Evolution in temporally varying environments will tend to favor types that have lower mean fitness in good times but that resist having very low fitness in bad times. We did not consider these important issues in our target article in favor of issues more directly relevant to the evolution of cooperation. As Equation 1 in section 4.1 depicts, in the context of multi-level selection, the ratio of group benefits to individual costs that can be favored by group selection depends upon the ratio of within-group to between-group heritable variation for the trait concerned.

\section{R14. The evidence is incomplete}

Commentators Mace \& Silva, Barclay \& Krupp, and Turchin \& Currie remark that the evidence supporting CGS is inadequate to support any strong claims for the utility of the framework. Mace \& Silva point out that the case for CGS to the exclusion of other explanations is hard to prove. We feel that there is no need to prove the case to the exclusion of all other processes that influence or may influence human behavior. Given that human societies are complex and diverse, we do not expect a single model derived from the CGS framework, or any other framework, to provide a complete and universal explanation. In many cases, competing hypotheses or models are not mutually exclusive.

Barclay \& Krupp say that we have established that CGS processes are plausible, but that we have not shown that a particular group trait was caused by cultural transmission and directly caused differences in group fitness. We did point to evidence that norms are transmitted (see sects. 3 and 4.2) and that the outcomes of competition are often explained by institutional and other cultural differences between groups (sect. 5). Institutions are group-level traits by their nature (see sect. R5 here). We have pointed to a small selection of concrete empirical cases in our article and additional ones in this response, but Barclay \& Krupp are right that in no particular case can we put all the pieces of a complete CGS explanation together. This is a serious problem in the science of evolution generally (Brandon 1994). Biologists know a lot about genetic transmission, mainly from laboratory studies, and a lot about the evolution of phenotypic characters in the wild, but in most cases, connecting genes to phenotypic characters has proven difficult. Many good studies of the transmission of norms have been done, and the role of institutions in inter-organizational competition has also been wellstudied. But it is hard to conceive of a study that would allow us to understand how a complex institution is built from the ground up by the social learning of norms. The complexity of cultural inheritance and of cultural evolution precludes easy progress on many important questions. Evolutionary biology is still a thriving scientific field 155 years after the Origin of Species; we do not think the study of cultural evolution will be a perfected science a century from now. 
References/Richerson et al.: Cultural group selection plays an essential role in explaining human cooperation

In this regard, we enthusiastically endorse the path forward painted by Turchin \& Currie. The gold standard in empirical inference has become fitting competing models to data using techniques such as Markov Chain Monte Carlo simulations and testing the goodness of fit of these models to data using Bayesian and information theoretic measures (e.g., Gerbault et al. 2014). Advances in genomics have furnished huge amounts of historical data on the course of evolution. Human languages, living and dead, have long been mined for human historical information, and to some extant non-linguistic data have been used as well (see sect. 3.2). As Turchin \& Currie suggest, archaeological and historical data can surely be assembled into databases that can support model-fitting and model-comparison approaches.

\section{R15. Conclusion}

Our commentators made many useful addenda to the main argument in the target article but also introduced a number of caveats. They retained varying degrees of skepticism about the reality of the CGS processes. However, none of the skeptics addressed the empirical foundations for applying the Darwinian syllogism to human groups, except in special cases. In the target article we presented evidence that:

1. Human groups often differ culturally.

2. Cultural variation is transmitted from generation to generation by social learning.

3. Success in intergroup competition is frequently determined by cultural differences.

We included only a small proportion of the vast amount of evidence that exists to support these statements. We attempted to select the best and admit that the quality of this evidence varies. However, consider the possibility of applying the CGS framework to non-human species, even the other great apes. Chimpanzee groups have appreciable amounts of cultural variation, including traditional differences in social customs like grooming (Whiten et al. 1999). Chimpanzees are comparatively good social learners, as judged by the standards of other non-human animals, albeit much less adept than human children (Dean et al. 2012; Whiten et al. 2009). Chimpanzee communities certainly compete vigorously. But so far as we are aware, there is no evidence that success in competition is influenced by a cultural trait. Some whale and dolphin species also have significant cultural traditions. Some group selection on them is conceivable and the current evidence is suggestive (Whitehead \& Rendell 2015). Most nonhuman social learning systems are considerably less sophisticated than those of chimpanzees and dolphins. No other vertebrate species has societies that compare to those of humans in size and organizational complexity. Certainly, skeptics about the importance of CGS processes in other species can raise strong doubts about their role. Only in humans is there abundant, if not always unassailable, evidence for all of the legs of the Darwinian syllogism operating at the group level.

\section{NOTE}

1. Krasnow \& Delton represent a particular, highly controversial brand of evolutionary psychology developed by Tooby \& Cosmides. It has become conventional to use capitals to distinguish the particular brand from the generic field. Accordingly, whenever "Evolutionary Psychology" appears capitalized in our text, we are referring to this term in Tooby \& Cosmides' sense.

\section{References}

[The letters "a" and " $r$ " before author's initials stand for target article and response references, respectively]

Aaldering, H. \& De Dreu, C. K. W. (2012) When doves fly high and hawks lay low: Constituent status differentials affect representative negotiation. Group Processes and Intergroup Relations 15:713-24. [CKWDD]

Acedo-Carmona, C. \& Gomila, A. (2013) Trust and cooperation: A new experimental approach. Annals of the New York Academy of Sciences 1299:77-83. [CA-C]

Acedo-Carmona, C. \& Gomila, A. (2014a) Diversidad étnica, clanes y familias en Ghana: Plataforma económica, social y cultural. In: Periferias, Fronteras y Diálogos. Actas del XIII Congreso de Antropología de la Federación de Asociaciones de Antropología del Estado Español, ed. N. Lundsteen, U. MartínezVeiga \& J. Palomera, pp. 2519-45. Universitat Rovira i Virgili. Available at: http://wwwa.fundacio.urv.cat/congres-antropologia/. [CA-C]

Acedo-Carmona, C. \& Gomila, A. (2014b) Personal trust increases cooperation beyond general trust. PLOS ONE 9(8):e105559. [CA-C]

Acedo-Carmona, C. \& Gomila, A. (2015) Trust matters: A cross-cultural comparison of Northern Ghana and Oaxaca groups. Frontiers in Psychology 6(661). doi: 10.3389/fpsyg.2015.00661. [CA-C]

Acemoglu, D., Johnson, S. \& Robinson, J. A. (2001) The colonial origins of comparative development: An empirical investigation. American Economic Review 91(5):1369-401. doi: 10.1257/aer.91.5.1369. [PH]

Acemoglu, D., Johnson, S. \& Robinson, J. A. (2002) Reversal of fortune: Geography and institutions in the making of the modern world income distribution. The Quarterly Journal of Economics 117(4):1231-94. doi: 10.1162/ 003355302320935025 . [PH]

Acemoglu, D., Johnson, S. \& Robinson, J. A. (2005) Institutions as a fundamental cause of long-run growth. In: Handbook of economic growth, vol. 1, Part A, ed. A. Philippe \& N. D. Steven, pp. 385-72. Elsevier. [PH]

Acerbi, A., Ghirlanda, S. \& Enquist, M. (2012) The logic of fashion cycles. PLoS One 7(3):e32541. [HH]

Akey, J. M. (2009) Constructing genomic maps of positive selection in humans: Where do we go from here? Genome Research 19(5):711-22. doi: 10.1101/ gr.086652.108. [PH]

Aktipis, C. A. (2004) Know when to walk away: Contingent movement and the evolution of cooperation. Journal of Theoretical Biology 231(2):249-60. [RMN]

Alcock, J. (1998) Animal behavior, 6th edition. Sinauer. [MMK]

Alcock, J. (2001) The triumph of sociobiology. Oxford University Press. [MMK] Alexander, R. D. (1987) The biology of moral systems. Aldine de Gruyter. [aPR] Algan, Y. \& Cahuc, P. (2010) Inherited trust and growth. The American Economic Review 100(5):2060-92. doi: 10.2307/41038755. [aPR]

Allison, A. C. (1964) Polymorphism and natural selection in human populations. Cold Spring Harbor Symposia on Quantitative Biology 29:137-49. doi: 10.1101/ sqb.1964.029.01.018. [aPR]

Allport, G. W. (1954) The nature of prejudice. Addison-Wesley. [CKWDD]

Alvard, M. (2011) Genetic and cultural kinship among the Lamaleran whale hunters. Human Nature 22(1-2):89-107. doi: 10.1007/s12110-011-9104-x. [JH]

Alvard, M. S. (2003) The adaptive nature of culture. Evolutionary Anthropology 12:136-49. [CA-C]

Andersson, C., Törnberg, A. \& Törnberg, P. (2014) An evolutionary developmental approach to cultural evolution. Current Anthropology 55(2):154-74. [AF]
[A

André, J.-B. \& Morin, O. (2011) Questioning the cultural evolution of altruism. Journal of Evolutionary Biology 24(12):2531-42. [OM]

Aoki, M. (2001) Toward a comparative institutional analysis. MIT Press. [aPR]

Archer, J. (1997) Why do people love their pets? Evolution and Human Behavior 18(4):237-59. [HH]

Armentano, D. T. (1986) Antitrust policy: The case for repeal. Cato Institute. $\quad[\mathrm{PH}]$

Ashforth, B. E., Harrison, S. H. \& Corley, K. G. (2008) Identification in organizations: An examination of four fundamental questions. Journal of Management 34(3):325-74. doi: 10.1177/0149206308316059. [aPR]

Ashraf, Q. \& Galor, O. (2013) The "Out of Africa" hypothesis, human genetic diversity, and comparative economic development. American Economic Review 103(1):1-46. doi: 10.1257/aer.103.1.1. [PH]

Atran, S. (2002) In gods we trust. Oxford University Press. [aPR]

Atran, S. (2010) Talking to the enemy: Faith, brotherhood, and the (un)making of terrorists. HarperCollins. [aPR] 
References/Richerson et al.: Cultural group selection plays an essential role in explaining human cooperation

Atran, S. \& Norenzayan, A. (2005) Religion's evolutionary landscape: Counterintuition, commitment, compassion, communion. Behavioral and Brain Sciences 27(6):713-70. doi: 10.1017/s0140525x04000172. [aPR]

Aunger, R., ed. (2000) Darwinizing culture: The status of memetics as a science. Oxford University Press. [AW]

Ayala, F. J. (2010) The difference of being human: Morality. Proceedings of the National Academy of Sciences USA 107(Suppl. 2):8897-901. [aPR, JSC]

Balikci, A. (1970) The Netsilik Eskimo. Doubleday. [DR]

Balliet, D., Wu, Y. \& De Dreu, C. K. W. (2014) In-group favoritism and cooperation: A meta-analysis. Psychological Bulletin 140:1556-81. [CKWDD]

Barclay, P. (2012) Proximate and ultimate causes of strong reciprocity and punishment. Behavioral and Brain Sciences 35(1):16-17. [PB]

Barclay, P. (2013) Strategies for cooperation in biological markets, especially for humans. Evolution and Human Behavior 34(3):164-75. doi: 10.1016/j.evolhumbehav.2013.02.002. [JH]

Barclay, P. \& Van Vugt, M. (2015) The evolutionary psychology of human prosociality: Adaptations, mistakes, and byproducts. In: Oxford handbook of prosocial behavior, ed. D. Schroeder \& W. Graziano, pp. 37-60. Oxford University Press. [PB]

Barnett, W. P. \& McKendrick, D. G. (2004) Why are some organizations more competitive than others? Evidence from a changing global market. Administrative Science Quarterly 49(4):535-71. [aPR]

Barnett, W. P. \& Pontikes, E. G. (2008) The Red Queen, success bias, and organizational inertia. Management Science 54(7):1237-51. doi: 10.1287/ mnsc.1070.0808. [aPR]

Barrett, H. C. (2015) The shape of thought: How mental adaptations evolve. Oxford University Press. [rPR]

Batson, C. D. (2011) Altruism in humans. Oxford University Press. [arPR]

Baum, W. M. (2012) Rethinking reinforcement: Allocation, induction, and contingency. Journal of the Experimental Analysis of Behavior 97(1):101-24. [CSi]

Baum, W. M. (2015) Driven by consequences: The multiscale molar view of choice. Managerial and Decision Economics 9999: doi: 10.1002/mde.2713. [CSi]

Baum, W. M., Paciotti, B., Richerson, P., Lubell, M. \& McElreath, R. (2012) Cooperation due to cultural norms, not individual reputation. Behavioural Processes 91(1):90-93. doi: 10.1016/j.beproc.2012.06.001. [arPR]

Baum, W. M., Richerson, P. J., Efferson, C. M. \& Paciotti, B. M. (2004) An experimental model of cultural evolution including traditions of rule giving and rule following. Evolution and Human Behavior 25:305-26. [aPR]

Baumard, N., Hyafil, A., Morris, I. \& Boyer, P. (2015) Increased affluence explains the emergence of ascetic wisdoms and moralizing religions. Current Biology 25(1):10-15. doi: 10.1016/j.cub.2014.10.063. [JH]

Baumgartner, T., Heinrichs, M., Vonlanthen, A., Fischbacher, U. \& Fehr, E. (2008) Oxytocin shapes the neural circuitry of trust and trust adaptation in humans. Neuron 58(4):639-50. [JSC]

Beebe, B. \& Lachmann, F. M. (2014) The origins of attachment. Routledge. [JSC]

Belin, P., Zatorre, R. J., Lafaille, P., Ahad, P. \& Pike, B. (2000) Voice-selective areas in human auditory cortex. Nature 403:309-12. [HG]

Bell, A. V., Richerson, P. J. \& McElreath, R. (2009) Culture rather than genes provides greater scope for the evolution of large-scale human prosociality. Proceedings of the National Academy of Sciences USA 106(42):17671-74. doi: 10.1073/pnas.0903232106. [arPR, JH, RMR]

Bellah, R. N. (2011) Religion in human evolution: From the Paleolithic to the Axial Age. Harvard University Press. [PT]

Belyaev, D. K. (1979) Destabilizing selection as a factor in domestication. Journal of Heredity 70:301-308. [HG]

Belyaev, D. K., Plyusnina, I. Z. \& Trut, L. N. (1985) Domestication of the silver fox (Vulpes fulvus Desm): Changes in physiological boundaries of the sensitive period of primary socialization. Applied Animal Behavior Science 13:359-70. [JSC]

Bernard, M., Dreber, A., Strimling, P. \& Eriksson, K. (2013) The subgroup problem When can binding voting on extractions from a common pool resource overcome the tragedy of the commons? Journal of Economic Behavior and Organization 91:122-30. [DA]

Bhugra, D. \& Becker, M. A. (2005) Migration, cultural bereavement and cultural identity. World Psychiatry 4(1):18-24. [CA-C]

Binder, J. R., Frost, J. A., Hammeke, T. A., Cox, R. W., Rao, S. M. \& Prieto, T. (1997) Human brain language areas identified by functional magnetic resonance imaging. Journal of Neuroscience 17:353-62. [HG]

Bingham, P. M. (1999) Human uniqueness: A general theory. Quarterly Review of Biology 74(2):133-69. [HG]

Birch, J. \& Okasha, S. (2015) Kin selection and its critics. BioScience 65:22-32. [PB]

Boehm, C. (1984) Blood revenge. The anthropology of feuding in Montenegro and other tribal societies. University Press of Kansas. [rPR]

Boehm, C. (1997) Impact of the human egalitarian syndrome on Darwinian selection mechanics. The American Naturalist 150(Suppl.):S100-21. [aPR]

Boehm, C. (2012) Moral origins: The evolution of virtue, altruism, and shame. Basic Books. [aPR
Boesch, C. (1991) Teaching among wild chimpanzees. Animal Behaviour 41:53032. [JMB]

Boldrin, M. \& Levine, D. K. (2008) Against intellectual monopoly. Cambridge University Press. $[\mathrm{PH}]$

Bolhuis, J. J., Brown, G. R., Richardson, R. C. \& Laland, K. N. (2011) Darwin in mind: New opportunities for evolutionary psychology. PLoS Biology 9(7): e1001109. doi: 10.1371/journal.pbio.1001109. [rPR]

Bolton, M. K. (1993) Imitation versus innovation: Lessons to be learned from the Japanese. Organizational Dynamics 21(3):30-45. [aPR]

Bornstein, G. (2003) Intergroup conflict: Individual, group, and collective interests. Personality and social psychology review 7(2):129-45. [CKWDD]

Bouckaert, R., Lemey, P., Dunn, M., Greenhill, S. J., Alekseyenko, A. V., Drummond, A. J., Gray, R. D., Suchard, M. A. \& Atkinson, Q. D. (2012) Mapping the origins and expansion of the Indo-European language family. Science 337 (6097):957-60. doi: 10.1126/science.1219669. [aPR]

Bowlby, J. (1946) Psychology and democracy. Political Quarterly 17:61-76. [JSC]

Bowles, S. (2006) Group competition, reproductive leveling, and the evolution of human altruism. Science 314:1569-72. [aPR]

Bowles, S. \& Gintis, H. (2011) A cooperative species: Human reciprocity and its evolution. Princeton University Press. [aPR, CKWDD, HG]

Boyd, R., Gintis, H., Bowles, S. \& Richerson, P. J. (2003) The evolution of altruistic punishment. Proceedings of the National Academy of Sciences USA 100:353135. $[\mathrm{aPR}]$

Boyd, R. \& Lorderbaum, J. P. (1987) No pure strategy is evolutionarily stable in the repeated Prisoner's Dilemma game. Nature 32(6117):58-59. [JH]

Boyd, R. \& Mathew, S. (2015) The evolution of language may require third-party monitoring and sanctions. Evolution and Human Behavior 36(6):475-79. [JH]

Boyd, R. \& Richerson, P. J. (1985) Culture and the evolutionary process. University of Chicago Press. [arPR, MMK]

Boyd, R. \& Richerson, P. J. (1990) Group selection among alternative evolutionarily stable strategies. Journal of Theoretical Biology 145(3):331-42. [OM]

Boyd, R. \& Richerson, P. J. (1992a) How microevolutionary processes give rise to history. In: History and evolution, ed. M. H. Nitecki \& D. V. Nitecki, pp. 179209. State University of New York Press. [rPR]

Boyd, R. \& Richerson, P. J. (1992b) Punishment allows the evolution of cooperation (or anything else) in sizable groups. Ethology and Sociobiology 13(3):171-95. [arPR]

Boyd, R. \& Richerson, P. J. (2002) Group beneficial norms can spread rapidly in a structured population. Journal of Theoretical Biology 215:287-96. [aPR]

Boyd, R. \& Richerson, P. J. (2009) Voting with your feet: Payoff biased migration and the evolution of group beneficial behavior. Journal of Theoretical Biology 257 (2):331-39. doi: 10.1016/j.jtbi.2008.12.007. [aPR, OM]

Boyd, R. \& Richerson, P. J. (2010) Transmission coupling mechanisms: Cultural group selection. Philosophical Transactions of the Royal Society of London, Series B: Biological Sciences 365(1559):3787-95. doi:10.1098/rstb.2010.0046. [MS]

Boyd, R., Richerson, P. J. \& Henrich, J. (2011) Rapid cultural adaptation can facilitate the evolution of large-scale cooperation. Behavioral Ecology and Sociobiology 65:431-44. [aPR]

Boyer, P. (2001) Religion explained: The evolutionary origins of religious thought. Basic Books. [aPR]

Boyer, P. \& Petersen, M. B. (2011) The naturalness of (many) social institutions Journal of Institutional Economics 8(1):1-25. [JT]

Bradshaw, J. (2013) Cat sense: How the new feline science can make you a better friend to your pet. Basic Books. [HH]

Brandon, R. N. (1994) Theory and experiment in evolutionary biology. Synthese 99 (1):59-73. doi: 10.2307/20117886. [rPR]

Brewer, M. B. (1999) The psychology of prejudice: Ingroup love or outgroup hate? Journal of Social Issues 55:429-44. [CKWDD]

Brewer, M. B. (2007) The importance of being we: Human nature and intergroup relations. American Psychologist 62(8):728-38. doi: dx.doi.org/10.1037/0003066X.62.8.728. [arPR]

Brooks, B. A., Hoff, K. \& Pandey, P. (2014) Insult versus accident: Caste culture and the efficiency of coordination. Available at: http://faculty.chicagobooth.edu/ workshops/micro/past/pdf/brooksinsultvsaccident.pdf. [PH]

Brown, G. \& Richerson, P. (2014) Applying evolutionary theory to human behaviour: Past differences and current debates. Journal of Bioeconomics 16(2):105-28. doi: 10.1007/s10818-013-9166-4. [aPR]

Brown, S., Savage, P. E., Ko, A. M., Stoneking, M., Ko, Y. C., Loo, J. H. \& Trejaut, J A. (2014) Correlations in the population structure of music, genes and language. Proceedings of the Royal Society B: Biological Sciences 281(1774):e20132072. doi: 10.1098/rspb.2013.2072. [RMR]

Burkart, J. M., Allon, O., Amici, F., Fichtel, C., Finkenwirth, C., Heschl, A., Huber J., Isler, K., Kosonen, Z. K., Martins, E., Meulman, E. J., Richiger, R., Rueth, K., Spillmann, B., Wiesendanger, S. \& van Schaik, C. P. (2014) The evolutionary origin of human hyper-cooperation. Nature Communications 5, Article no. 4747. (Online article, published August 27, 2014). [JMB] 
Burkart, J. M., Hrdy, S. B. \& Van Schaik, C. P. (2009) Cooperative breeding and human cognitive evolution. Evolutionary Anthropology 18:175-86. [aPR, $\mathrm{JMB}]$

Burkart, J. M. \& van Schaik, C. P. (2010) Cognitive consequences of cooperative breeding in primates. Animal Cognition 13:1-19. [JMB]

Buss, D. M., ed. (2005) The handbook of evolutionary psychology. Wiley. [MMK]

Buss, D. M. (2014) Evolutionary psychology, 5th edition. Pearson. [MMK, rPR]

Buss, D. M. \& Reeve, H. K. (2003) Evolutionary psychology and developmental dynamics. Psychological Bulletin 129:848-53. [MMK]

Buttelmann, D. \& Böhm, R. (2014) The ontogeny of the motivation that underlies ingroup bias. Psychological Science 25:921-27. [CKWDD]

Byars, S. G., Ewbank, D., Govindaraju, D. R. \& Stearns, S. C. (2010) Natural selection in a contemporary human population. Proceedings of the National Academy of Science USA 107:1787-92. doi: 10.1073/pnas.0906199106. [aPR]

Byrne, R. W. \& Rapaport, L. G. (2011) What are we learning from teaching? Animal Behaviour 82(5):1207-11. doi: 10.1016/j.anbehav.2011.08.018. [JMB]

Calvin, W. H. (1983) A stone's throw and its launch window: Timing precision and its implications for language and hominid brains.Journal of Theoretical Biology 104:121-35. [HG]

Campbell, D. T. (1975) On the conflicts between biological and social evolution and between psychology and moral tradition. American Psychologist 30 (12):1103-26. [aPR]

Capraro, V. \& Cococcioni, G. (2015) Social setting, intuition, and experience in lab experiments interact to shape cooperative decision-making. Available at Social Science Research Network (SSRN): http://ssrn.com/abstract=2559182. [DA]

Carballo, D. M., Roscoe, P. \& Feinman, G. M. (2014) Cooperation and collective action in the cultural evolution of complex societies. Journal of Archaeological Method and Theory 21:98-133. [PT]

Carey, S. (2009) The origin of concepts. Oxford University Press. [aPR]

Carneiro, R. L. (1970) A theory of the origin of the state. Science 169:733-38. [PT]

Cassidy, J. (2008) The nature of the child's ties. In: Handbook of attachment: Theory, research, and clinical applications, ed. J. Cassidy \& P. R. Shaver, pp. 3-22. Guilford Press. [JSC]

Cavalli-Sforza, L. L. \& Feldman, M. W. (1981) Cultural transmission and evolution: A quantitative approach. Princeton University Press. [arPR]

Cavalli-Sforza, L. L., Menozzi, P. \& Piazza, A. (1994) The history and geography of human genes. Princeton University Press. [aPR]

Cavalli-Sforza, L. L., Piazza, A., Menozzi, P. \& Mountain, J. (1988) Reconstruction of human evolution: Bringing together genetic, archaeological, and linguistic data. Proceedings of the National Academy of Sciences USA 85(16):6002-6006. [aPR]

Chanda, A., Cook, C. J. \& Putterman, L. (2014) Persistence of fortune: Accounting for population movements, there was no post-Columbian reversal. American Economic Journal: Macroeconomics 6(3):1-28. doi: 10.1257/mac.6.3.1. [PH]

Chapais, B. (2008) Primeval kinship: How pair-bonding gave birth to human society. Harvard University Press. [aPR]

Chen, Y. \& Xin Li, S. (2009) Group identity and social preferences. The American Economic Review 99(1):431-57. [CA-C]

Chiao, J. Y. \& Blizinsky, K. D. (2010) Culture-gene coevolution of individualismcollectivism and the serotonin transporter gene. Proceedings of the Royal Society B: Biological Sciences 277(1681):529-37. doi: 10.1098/rspb.2009.1650. [aPR]

Chisholm, J. S. (2003) Uncertainty, contingency and attachment: A life history theory of theory of mind. In: From mating to mentality: Evaluating evolutionary psychology, ed. K. Sterelny \& J. Fitness, pp. 125-53. Psychology Press. [JSC]

Choi, J.-K. \& Bowles, S. (2007) The coevolution of parochial altruism and war. Science 318(5850):636-40. [aPR]

Cieri, R. L., Churchill, S. E., Franciscus, R. G., Tan, J. \& Hare, B. (2014) Craniofacial feminization, social tolerance, and the origins of behavioral modernity. Current Anthropology 55(4):419-43. [HG]

Cikara, M., Bruneau, E., Van Bavel, J. J. \& Saxe, R. (2014) Their pain gives us pleasure: How intergroup dynamics shape empathic failures and counter-empathic responses. Journal of Experimental Social Psychology 55:110-25. doi:10.1016/j.jesp.2014.06.007. [JMV]

Cikara, M., Bruneau, E. G. \& Saxe, R. R. (2011) Us and them: Intergroup failures of empathy. Current Directions in Psychological Science 20(3):149-53. doi:10.1177/0963721411408713. [JMV]

Clark, J. D. \& Brown, K. S. (2001) The Twin Rivers Kopje, Zambia: Stratigraphy, fauna, and artefact assemblages from the 1954 and 1956 excavations. Journal of Archaeological Science 28(3):305-30. [AF]

Clutton-Brock, T. (2009) Cooperation between non-kin in animal societies. Nature $462(7269): 51-57 . \quad[\mathrm{JH}]$

Cochran, G. \& Harpending, H. (2009) The 10,000 year explosion: How civilization accelerated human evolution. Basic Books. [aPR]

Collard, M., Edinborough, K., Shennan, S. \& Thomas, M. G. (2010) Radiocarbon evidence indicates that migrants introduced farming to Britain. Journal of $\mathrm{A} r$ chaeological Science 37(4):866-70. doi: dx.doi.org/10.1016/j.jas.2009.11.016. [aPR]
Cone, J. \& Rand, D. G. (2014) Time pressure increases cooperation in competitively framed social dilemmas. PLOS ONE 9(12):e115756. doi: 10.1371/journal. pone.0115756. [DA]

Cook, C. J. (2014) The role of lactase persistence in precolonial development. Journal of Economic Growth 19(4):369-406. doi: 10.1007/s10887-0149109-5. [PH]

Cooper, R. W. (1999) Coordination games: Complementarities and macroeconomics. Cambridge University Press. [aPR]

Cornelissen, G., Dewitte, S. \& Warlop, L. (2011) Are social value orientations expressed automatically? Decision making in the Dictator Game. Personality and Social Psychology Bulletin 37(8):1080-90. [DA]

Corriveau, K. H., Chen, E. E. \& Harris, P. L. (2014) Judgments about fact and fiction by children from religious and nonreligious backgrounds. Cognitive Science 39(2):353-82. [SS]

Corriveau, K. H. \& Harris, P. L. (2009) Preschoolers continue to trust a more accurate informant 1 week after exposure to accuracy information. Developmental Science 12(1):188-93. [SS]

Corriveau, K. H., Kinzler, K. D. \& Harris, P. L. (2013) Accuracy trumps accent in children's endorsement of object labels. Developmental psychology $49(3): 470 . \quad[\mathrm{SS}]$

Corriveau, K. H., Pickard, K. \& Harris, P. L. (2011) Preschoolers trust particular informants when learning new names and new morphological forms. British Journal of Developmental Psychology 29(1):46-63. [SS]

Cosmides, L. \& Tooby, J. (2001) Unravelling the enigma of human intelligence: Evolutionary psychology and the multimodular mind. In: The evolution of intelligence, ed. R. J. Sternberg \& J. C. Kaufman, pp. 145-99. Erlbaum. [aPR]

Coussi-Korbel, S. \& Fragaszy, D. M. (1995) On the relation between social dynamics and social learning. Animal Behaviour 50:1441-53. [JMB]

Cronk, L. \& Leech, B. H. (2013) Meeting at Grand Central: Understanding the evolutionary roots of cooperation. Princeton University Press. [aPR]

Csibra, G. \& Gergely, G. (2011) Natural pedagogy as an evolutionary adaptation. Philosophical Transactions of the Royal Society B 366:1149-57. [aPR]

Currie, T. E., Greenhill, S. J., Gray, R. D., Hasegawa, T. \& Mace, R. (2010) Rise and fall of political complexity in island South-East Asia and the Pacific. Nature 467 (7317):801-804. [arPR, RM]

Currie, T. E. \& Mace, R. (2009) Political complexity predicts the spread of ethnolinguistic groups. Proceedings of the National Academy of Sciences USA 106(18):7339-44. doi: 10.1073/pnas.0804698106. [aPR, RM]

Curtin, P. D. (1984) Cross-cultural trade in world history. Cambridge University Press. $[\mathrm{aPR}]$

Daly, M. \& Wilson, M. (1988) Homicide. Aldine de Gruyter. [MMK]

Daly, M. \& Wilson, M. (2010) Cultural inertia, economic incentives, and the persistence of "Southern violence." In: Evolution, culture, and the human mind, ed. M. Schaller, A. Norenzayan, S. Heine, T. Yamagishi \& T. Kameda, pp. 229-41. Taylor \& Francis. $\quad[\mathrm{PB}]$

Damasio, A. (1994) Descartes' error: Emotion, reason, and the human brain Putnam. [JSC]

Damasio, A. (1999) The feeling of what happens: Body and emotion in the making of consciousness. Harcourt Brace. [JSC]

Darwin, C. (1871) The descent of man, and selection in relation to sex. Murray. [HG]

Darwin, C. (1874) The descent of man and selection in relation to sex, 2nd edition. American Home Library. [CSi, arPR]

Davies, N. (2011) Vanished kingdoms: The rise and fall of states and nations. Viking. $\quad[\mathrm{rPR}]$

Dawkins, R. (1976) The selfish gene. Oxford University Press. [AW]

Deacon, R. \& Shapiro, P. (1975) Private preference for collective goods revealed through voting on referenda. The American Economic Review 65(5):943-55. [DA]

Dean, L. G., Kendal, R. L., Schapiro, S. J., Thierry, B. \& Laland, K. N. (2012) Identification of the social and cognitive processes underlying human cumulative culture. Science 335(6072):1114-18. doi: 10.1126/science.1213969. [arPR]

De Dreu, C. K. W. (2010) Social conflict: The emergence and consequences of struggle and negotiation. In: Handbook of social psychology, vol. 2, 5th edition, ed. S. T. Fiske, D. T. Gilbert, \& G. Lindzey, pp. 983-1023. Wiley. [CKWDD]

De Dreu, C. K. W., Balliet, D. \& Halevy, N. (2014) Parochial cooperation in humans: Forms and functions of self-sacrifice in intergroup competition and conflict. In Advances in motivational science, vol. 1, ed. A. J. Elliot, pp. 1-47. Elsevier. [CKWDD]

De Dreu, C. K. W. \& Carnevale, P. J. D. (2003) Motivational bases of information processing and strategy in conflict and negotiation. In: Advances in experimental social psychology, vol. 35, ed. M. P. Zanna, pp. 235-91. Academic Press. [CKWDD]

De Dreu, C. K. W., Greer, L. L., Handgraaf, M. J. J., Shalvi, S., Van Kleef, G. A., Baas, M., Ten Velden, F. S., Van Dijk, E. \& Feith, S. W. W. (2010) The neuropeptide oxytocin regulates parochial altruism in intergroup conflict among humans. Science 328:1408-11. [CKWDD] 
De Dreu, C. K. W., Greer, L. L., Van Kleef, G. A., Shalvi, S. \& Handgraaf, M. J. J (2011) Oxytocin promotes human ethnocentrism. Proceedings of the National Academy of Sciences USA 108:1262-66. [CKWDD]

Dell'Mour, V., Range, F. \& Huber, L. (2009) Social learning and mother's behavior in manipulative tasks in infant marmosets. American Journal of Primatology 71(6):503-509. [JMB]

Delton, A. W. \& Krasnow, M. M. (2014) An independent replication that the evolution of direct reciprocity under uncertainty explains one-shot cooperation: Commentary on Zefferman. Evolution and Human Behavior 35(6):547-48. doi: http://dx.doi.org/10.1016/j.evolhumbehav.2014.07.004. [rPR]

Delton, A. W., Krasnow, M. M., Cosmides, L. \& Tooby, J. (2011) Evolution of direct reciprocity under uncertainty can explain human generosity in one-shot encounters. Proceedings of the National Academy of Sciences USA 108(32):1333540. doi: 10.1073/pnas.1102131108. [arPR, JH, JT]

Derex, M., Godelle, B. \& Raymond, M. (2014) How does competition affect the transmission of information? Evolution and Human Behavior 35(2):89-95. doi: dx.doi.org/10.1016/j.evolhumbehav.2013.11.001. [aPR]

Derfus, P., Maggitti, P., Grimm, C. \& Smith, K. (2008) The Red Queen effect: Competitive actions and firm performance. The Academy of Management Journal 51(1):61-80. [aPR]

Deutsch, M. (1973) The resolution of conflict. Academic Press. [CKWDD]

Diamond, J. (1993) New Guineans and their natural world. In: The biophilia hypothesis, ed. S. R. Kellert \& E. O. Wilson, pp. 251-71. Island Press. [HH]

Doebel, S., Koenig, M. A. \& Rowell, S. (2011) Young children detect logical inconsistency. Poster presented at the 2011 Biennial Meeting of the Society for Research in Child Development, Montreal, Quebec, Canada, March 31-April 2, 2011. [SS]

Donald, M. (1991) Origins of the modern mind: Three stages in the evolution of culture and cognition. Harvard University Press. [aPR]

Dreber, A., Fudenberg, D., Levine, D. K. \& Rand, D. G. (2014) Altruism and selfcontrol. Available at Social Science Research Network (SSRN): http://ssrn.com/ abstract $=2477454 . \quad[\mathrm{DA}]$

Dunham, Y., Baron, A. S. \& Banaji, M. R. (2008) The development of implicit intergroup cognition. Trends in Cognitive Sciences 12:248-53. [aPR]

Durham, W. H. (1991) Coevolution: Genes, culture, and human diversity. Stanford University Press. [aPR]

Durkheim, E. (1912) The elementary forms of the religious life. Allen \& Unwin. $\quad$ [aPR]

Eccleston, C. \& Crombez, G. (1999) Pain demands attention: A cognitive-affective model on the interruptive function of pain. Psychological Bulletin 125:35666. [JMV]

Edgerton, R. B. (1971) The individual in cultural adaptation: A study of four East African peoples. University of California Press. [aPR, JH]

Efferson, C., Lalive, R. \& Fehr, E. (2008) The coevolution of cultural groups and ingroup favoritism. Science 321:1844-49. [aPR]

Eisenberger, N. I. \& Cole, S. W. (2012) Social neuroscience and health: Neurophysiological mechanisms linking social ties with physical health. Nature Neuroscience 15(5):669-74. doi:10.1038/nn.3086. [JMV]

Eldakar, O. T. \& Wilson, D. S. (2008) Selfishness as second order altruism. Proceedings of the National Academy of Sciences USA 105(19):6982-86. [aPR]

Ellingsen, T., Herrmann, B., Nowak, M. A., Rand, D. G. \& Tarnita, C. E. (2012) Civic capital in two cultures: The nature of cooperation in Romania and USA. CESifo Working Paper: Behavioural Economics. Available at Social Science Research Network (SSRN): http://ssrn.com/abstract=2179575. [DA]

Endler, J. A. (1986) Natural selection in the wild. Princeton University Press. [aPR]

Erdal, D. \& Whiten, A. (1996) Egalitarianism and Machiavellian intelligence in human evolution. In: Modelling the early human mind, ed. P. Mellars \& K. Gibson, pp. 139-50. McDonald Institute Monographs. [AW]

Evans, N. \& Levinson, S. C. (2009) The myth of language universals: Language diversity and its importance for cognitive science. Behavioral and Brain Sciences 32(5):429-92. [aPR]

Evans, N. \& McConvell, P. (1998) The enigma of Pama-Nyungan expansion in Australia. In: Archaeology and Language II: Correlating archaeological and linguistic hypotheses, ed. R. Blench \& M. Spriggs, pp. 174-91. Routledge. [JH]

Evans-Pritchard, E. E. (1940) The Nuer: A description of the modes of livelihood and political institutions of a Nilotic people. Clarendon Press. [DR, rPR]

Falk, A. \& Zehnder, C. (2013) A city-wide experiment on trust discrimination. Journal of Public Economics 100: 15-27. [SL]

Fearon, R. P., Bakermans-Kranenburg, M. J., van IJzendoorn, M. H., Lapsley, A.-M. \& Roisman, G. I. (2010) The significance of insecure attachment and disorganization in the development of children's externalizing behavior: A meta-analytic study. Child Development 81:435-56. [JSC]

Fehr, E. \& Fischbacher, U. (2003) The nature of human altruism. Nature 425:784-91. [OM]

Fehr, E. \& Gächter, S. (2002) Altruistic punishment in humans. Nature 415 (6868):137-40. [aPR]

Fehr, E. \& Henrich, J. (2003) Is strong reciprocity a maladaptation? On the evolutionary foundations of human altruism. In: Genetic and cultural evolution of cooperation, ed. P. Hammerstein, pp. 55-82. MIT Press. [aPR]
Feldman, M. W. \& Laland, K. N. (1996) Gene-culture coevolutionary theory. Trends in Ecology and Evolution 11(11):453-57. doi: 10.1016/0169-5347(96)10052-5 [aPR]

Finke, R. \& Stark, R. (1992) The Churching of America, 1776-1990: Winners and losers in our religious economy. Rutgers University Press. [aPR]

Fischer, D. H. (1989) Albion's seed: Four British folkways in America. Oxford University Press. [rPR]

Fiske, A. (1992) The four elementary forms of sociality: Framework for a unified theory of social relations. Psychological Review 99(4):689-23. [JH]

Flack, J. C., de Waal, F. B. M. \& Krakauer, D. C. (2005) Social structure, robustness, and policing cost in a cognitively sophisticated species. The American Naturalist 165(5):E126-39. doi:10.1086/429277. [MS]

Flack, J. C., Girvan, M., de Waal, F. B. M. \& Krakauer, D. C. (2006) Policing stabilizes construction of social niches in primates. Nature 439(7075):426-29. doi: 10.1038/nature04326. [MS]

Flegr, J. (2010) Elastic, not plastic species: Frozen plasticity theory and the origin of adaptive evolution in sexually reproducing organisms. Biology Direct 5(1):2. doi: $10.1186 / 1745-6150-5-2 . \quad[\mathrm{PH}]$

Flinn, M. V. \& Alexander, R. D. (2007) Runaway social selection in human evolution. In: The evolution of mind: Fundamental questions and controversies, ed. S. W. Gangestad \& J. A. Simpson, pp. 249-55. Guilford Press. [RMN]

Foley, R. \& Gamble, C. (2009) The ecology of social transitions in human evolution. Philosophical Transactions of the Royal Society, B 364:3267-79. [CA-C]
[C

Fonagy, P., Gergely, G. \& Target, M. (2007) The parent-infant dyad and the construction of the subjective self. Journal of Child Psychology and Psychiatry 48:288-28. [JSC]

Fonagy, P. \& Target, M. (1997) Attachment and reflective function: Their role in self-organization. Development and Psychopathology 9(4):679-700. [JSC]

Foster, G. M. (1960) Culture and conquest: America's Spanish heritage. WennerGren Foundation for Anthropological Research. [rPR]

Frank, S. A. (2006) Social selection. In: Evolutionary genetics: Concepts and case studies, ed. C. W. Fox \& J. B. Wolf, pp. 350-63. Oxford University Press. [RMN]

Fuentes, A. (2015) Integrative anthropology and the human niche: Towards a contemporary approach to human evolution. American Anthropologist 117 (2):302-15. doi: 10.1111/aman.12248. [AF]

Fukuyama, F. (2011) The origins of political order: From prehuman times to the French Revolution. Farrar, Straus and Giroux. [aPR]

Gächter, S., Herrmann, B. \& Thöni, C. (2010) Culture and cooperation. Philosophical Transactions of the Royal Society B: Biological Sciences 365 (1553):2651-61. [DA]

Gambetta, D. (1993) The Sicilian Mafia: The business of private protection. Harvard University Press. [aPR]

Gardner, A. \& Grafen, A. (2009) Capturing the superorganism: A formal theory of group adaptation. Journal of Evolutionary Biology 22:659-71. [PB]

Gardner, T. M. (2003) In the trenches at the talent wars: An examination of competitive interaction for human resources in the software industry. CAHRS Working Paper Series. Cornell University, School of Industrial and Labor Relations. [aPR]

Gat, A. (2008) War in human civilization. Oxford University Press. [aPR]

Geary, D. C. (2010) Male, female: The evolution of human sex differences, 2nd edition. American Psychological Association. [JMV]

Geary, D. C., Byrd-Craven, J., Hoard, M. K., Vigil, J. \& Numtee, C. (2003) Evolution and development of boys' social behavior. Developmental Review 23:444-70. [JMV]

Gelfand, M. J., Raver, J., Nishii, L., Leslie, L. \& Lun, J., Lim, B. C., Duan, L., Almaliach, A., Ang, S., Arnadottir, J., Aycan, Z., Boehnke, K., Boski, K., Cabecinhas, R., Chan, D., Chhokar, J., D’Amato, A., Ferrer, M., Fischlmayr, I. C., Fischer, R., Fulop, M., Georgas, J., Kashima, E. S., Kashima, Y., Kim, K. Lempereur, A., Marquez, P., Othman, R., Overlaet, B., Panagiotopoulou, P., Peltzer, K., Perez-Florizno, L. R., Ponomarenko, L., Realo, A., Schei, V., Schmitt, M., Smith, P. B., Soomro, N., Szabo, E., Taveesin, N., Toyama, M., Van de Vliert, E., Vohra, N., Ward, C. \& Yamaguchi, S. (2011) Differences between tight and loose cultures: A 33-nation study. Science 332:1100-104. $[\mathrm{MJG}]$

Gerbault, P., Allaby, R. G., Boivin, N., Rudzinski, A., Grimaldi, I. M., Pires, J. C., Vigueira, C. C., Dobney, K., Gremillion, K. J., Barton, L., Arroyo-Kalin, M., Purugganan, M. D., Rubio de Casas, R., Bollongino, R., Burger, J., Fuller, D. Q., Bradley, D. G., Balding, D. J., Richerson, P.J., Gilbert, M. T. P., Larsen, G. \& Thomas, M. G. (2014) Storytelling and story testing in domestication. Proceedings of the National Academy of Sciences USA 111(17):6159-64. doi: 10.1073/pnas.1400425111. [rPR]

Gervais, W. M., Willard, A. K., Norenzayan, A. \& Henrich, J. (2011) The cultural transmission of faith: Why innate intuitions are necessary, but insufficient, to explain religious belief. Religion 41(3):389-10. doi: 10.1080/ $0048721 x .2011 .604510 . \quad$ [arPR]

Ghirlanda, S., Acerbi, A. \& Herzog, H. (2014) Dog movie stars and dog breed popularity: A case study in media influence on choice. PLoS One 9(9): el06565. [HH] 
Ghirlanda, S., Acerbi, A., Herzog, H. \& Serpell, J. A. (2013) Fashion vs. function in cultural evolution: The case of dog breed popularity. PLoS One 8(9):e74770. $[\mathrm{HH}]$

Gibbons, A. (2014) How we tamed ourselves - and became modern. Science 346 (6208):405-406. [HG]

Gibson, C. C., Andersson, K., Ostrom, E. \& Shivakumar, S. (2005) The Samaritan's dilemma: The political economy of development aid. Oxford University Press. [ [rPR]

Gintis, H. (2008) Punishment and cooperation. Science 319(5868):1345-46. doi: 10.1126/science.1155333. [PH]

Gintis, H. (2014) Inclusive fitness and the sociobiology of the genome. Biology and Philosophy 29(4):477-15. [HG]

Gintis, H., van Schaik, C. \& Boehm, C. (2015) The evolutionary origins of human political systems. Current Anthropology 56(3):327-53. Available at: http://carta. anthropogeny.org/libraries/bibliography/zoon-politikon-the-evolutionary-originsof-human-political-systems. Also available at: http://people.umass.edu/ gintis. [HG]

Glaeser, E., La Porta, R., Lopez-de-Silanes, F. \& Shleifer, A. (2004) Do institutions cause growth? Journal of Economic Growth 9(3):271-303. doi: 10.1023/B: JOEG.0000038933.16398.ed. [PH]

Glantz, D. M. \& House, J. M. (1998) When Titans clashed: How the Red Army stopped Hitler. University Press of Kansas. [aPR]

Gliga, T. \& Csibra, G. (2009) One-year-old infants appreciate the referential nature of deictic gestures and words. Psychological Science 20(3):347-53. [SS]

Glowacki, L. \& Wrangham, R. W. (2013) The role of rewards in motivating participation in simple warfare. Human Nature 24(4):444-60. doi:10.1007/s12110013-9178-8. [MS]

Grafen, A. (1984) Natural selection, kin selection, and group selection. In: Behavioural ecology: An evolutionary approach, ed. J. R. Krebs \& N. B. Davies, Ch. 3, pp. 62-84. Sinauer. [HG]

Graham, J. \& Haidt, J. (2010) Beyond beliefs: Religions bind individuals into moral communities. Personality and Social Psychology Review 14(1):140-50. [aPR]

Gray, P. B. \& Young, S. M. (2011) Human-pet dynamics in cross-cultural perspective. Anthrozoös 24(1):17-30. [HH]

Gray, R. D., Drummond, A. J. \& Greenhill, S. J. (2009) Language phylogenies reveal expansion pulses and pauses in Pacific settlement. Science 323(5913):479-83. doi: 10.1126/science.1166858. [aPR]

Greenwald, A. G. \& Pettigrew, T. F. (2014) With malice toward none and charity for some: Ingroup favoritism enables discrimination. American Psychologist 69:669-84. [CKWDD]

Greif, A. (2006) Institutions and the path to the modern economy: Lessons from medieval trade. Cambridge University Press. [aPR]

Grove, M. \& Coward, F. (2008) From individual neurons to social brains. Cambridge Archaeological Journal 18:387-400. doi: 10.1017/S0959774308000437. [AF]

Guglielmino, C. R., Viganotti, C., Hewlett, B. \& Cavalli-Sforza, L. L. (1995) Cultural variation in Africa: Role of mechanisms of transmission and adaptation. Proceedings of the National Academy of Sciences 92(16):7585-89. [aPR]

Gürerk, Ö., Irlenbusch, B. \& Rockenbach, B. (2006) The competitive advantage of sanctioning institutions. Science 312(5770):108-11. doi: 10.1126/ science.1123633. [DA, aPR]

Gurven, M. (2004) Economic games among the Amazonian Tsimane: Exploring the roles of market access, costs of giving, and cooperation on pro-social game behavior. Experimental Economics 7(1):5-24. [SL]

Gurven, M., Zanolini, A. \& Schniter, E. (2008) Culture sometimes matters: Intracultural variation in pro-social behavior among Tsimane Amerindians. Journal of Economic Behavior and Organization 67(3-4):587-607. [SL]

Guthrie, R. D. (2005) The nature of Paleolithic art. Chicago University Press. [aPR]

Haak, W., Balanovsky, O., Sanchez, J. J., Koshel, S., Zaporozhchenko, V., Adler, C. J., Der Sarkissian, C. S. I., Brandt, G., Schwarz, C., Nicklisch, N., Dresely, V., Fritsch, B., Balanovska, E., Villems, R., Meller, H., Alt, K. W., Cooper, A. \& the Genographic Consortium (2010) Ancient DNA from European early Neolithic farmers reveals their Near Eastern affinities. PLoS Biology 8(11):e1000536. doi: 10.1371/journal.pbio.1000536 [aPR]

Hagen, E. H. \& Hammerstein, P. (2006) Game theory and human evolution: A critique of some recent interpretations of experimental games. Theoretical Population Biology 69(3):339-48. doi: 10.1016/.j.tpb.2005.09.005. [aPR, CKWDD]

Halevy, N., Bornstein, G. \& Sagiv, L. (2008) "In-group love" and "out-group hate" as motives for individual participation in intergroup conflict: A new game paradigm. Psychological Science 19:405-11. [CKWDD]

Halevy, N., Weisel, O. \& Bornstein, G. (2012) "In-group love" and "out-group hate" in repeated interaction between groups. Journal of Behavioral Decision Making 25:188-95. [CKWDD]

Hämäläinen, P. (2003) The rise and fall of Plains Indian horse cultures. The Journal of American History 90(3):833-62. [HH]

Hämäläinen, P. (2008) The Comanche empire. Yale University Press. [arPR]

Hamilton, W. D. (1975) Innate social aptitudes of man: An approach from evolutionary genetics. In: Biosocial Anthropology, ed. R. Fox, pp. 133-53. Malaby Press. [PB]
Hamlin, J. K., Wynn, K., Bloom, P. \& Mahajan, N. (2011) How infants and toddlers react to antisocial others. Proceedings of the National Academy of Sciences USA 108(50):19931-36. doi: 10.1073/pnas.1110306108. [aPR, DA]

Handley, L. J. L., Manica, A., Goudet, J. \& Balloux, F. (2007) Going the distance: Human population genetics in a clinal world. Trends in Genetics 23(9):432-39. doi: 10.1016/j.tig.2007.07.002. [RMR]

Hannan, M. T. \& Freeman, J. (1993) Organizational ecology: Harvard University Press. [aPR]

Hare, B., Plyusnina, I., Ignacio, N., Schepina, O., Stepika, A., Wrangham, R. \& Trut, L. (2005) Social cognitive evolution in captive foxes is a correlated by-product of experimental domestication. Current Biology 15:226-30. [JSC]

Harrington, J. \& Gelfand, M. J. (2014) Tightness-looseness across the U.S. 50 states Proceedings of the National Academy of Sciences USA 111:7990-95. [MJG]

Harris, P. L. (2012) Trusting what you're told: How children learn from others. Harvard University Press. [aPR]

Haslam, S. A. (2001) Psychology in organizations: The social identity approach. Sage. [arPR]

Haunschild, P. R. (1993) Interorganizational imitation: The impact of interlocks on corporate acquisition activity. Administrative Science Quarterly 38(4):564-92. doi: $10.2307 / 2393337$ [aPR]

Hauser, O. P., Rand, D. G., Peysakhovich, A. \& Nowak, M. A. (2014) Cooperating with the future. Nature 511:220-23. [DA]

Havlíček, J. \& Roberts, S. C. (2013) The perfume-body odour complex: An insightful model for culture-gene coevolution? In: Chemical signals in vertebrates 12, ed. M. L. East \& M. Dehnhard, pp. 185-95. Springer. [PH]

Hayes, C. (1951) The ape in our house. Harper. [aPR]

Henrich, J. (2004) Cultural group selection, co-evolutionary processes, and largescale cooperation. Journal of Economic Behavior and Organization 53:3-35. [CKWDD]

Henrich, J. (2004) Demography and cultural evolution: Why adaptive cultural processes produced maladaptive losses in Tasmania. American Antiquity 69 (2):197-214. [aPR]

Henrich, J. (2016) The secret of our success: How learning from others drove human evolution, domesticated our species, and made us smart. Princeton University Press. [JH]

Henrich, J., Bowles, S., Boyd, R. T., Hopfensitz, A., Richerson, P. J., Sigmund, K. Smith, E. A., Weissing, F. J. \& Young, H. P. (2003) Group report: The cultural and genetic evolution of human cooperation. In: Genetic and cultural evolution of cooperation, ed. P. Hammerstein, pp. 445-68. MIT Press. [aPR]

Henrich, J., Boyd, R., Bowles, S., Camerer, C., Fehr, E. \& Gintis, H. (2004) Foundations of human sociality: Economic experiments and ethnographic evidence from fifteen small-scale societies. Oxford University Press. [arPR, SL]

Henrich, J., Boyd, R., Bowles, S., Camerer, C., Fehr, E., Gintis, H. \& McElreath, R. (2001) In search of Homo economicus: Behavioral experiments in 15 smallscale societies. American Economic Review 73-78. [DA]

Henrich, J., Boyd, R., Bowles, S., Camerer, C. F., Fehr, E., Gintis, H., McElreath, R., Alvard, M., Barr, A., Ensminger, J., Henrich, N. S., Hill, K., Gil-White, F., Gurven, M., Marlowe, F. W., Patton, J. Q. \& Tracer, D. (2005) "Economic man" in cross-cultural perspective: Behavioral experiments in 15 small-scale societies. Behavioral and Brain Sciences 28(6):795-15. doi:10.1017/ S0140525X05000142. [RM]

Henrich, J., Boyd, R., McElreath, R., Gurven, M., Richerson, P. J., Ensminger, J., Alvard, M., Barr, A., Barrett, C., Bolyanatz, A., Camerer, C., Cardenas, J.-C., Fehr, E., Gintis, H. M., Gil-White, F., Gwako, E. L., Henrich, N., Hill, K., Lesorogol, C., Patton, J. Q, Marlowe, F. W., Tracer, D. P. \& Ziker, J. (2012a) Culture does account for variation in game behavior. Proceedings of the $\mathrm{Na}$ tional Academy of Sciences USA 109(2):E32-33. doi: 10.1073/ pnas.1118607109. [rPR]

Henrich, J., Boyd, R. \& Richerson, P. J. (2012b) The puzzle of monogamous marriage. Philosophical Transactions of the Royal Society B: Biological Sciences 367 (1589):657-69. doi: 10.1098/rstb.2011.0290. [aPR, JH]

Henrich, J., Ensminger, J., McElreath, R., Barr, A., Barrett, C., Bolyanatz, A., Cardenas, J. C., Gurven, M., Gwako, E., Henrich, N., Lesorogol, C., Marlowe, F., Tracer, D. P. \& Ziker, J. (2010a) Markets, religion, community size, and the evolution of fairness and punishment. Science 327(5972):1480-84. doi: 10.1126/ science.1182238. Available at: http://www.sciencemag.org/content/327/5972/ 1480. [arPR, SL, RM]

Henrich, J. \& Gil-White, F. J. (2001) The evolution of prestige - Freely conferred deference as a mechanism for enhancing the benefits of cultural transmission. Evolution and Human Behavior 22(3):165-96. [rPR]

Henrich, J., Heine, S. J. \& Norenzayan, A. (2010b) The weirdest people in the world? Behavioral and Brain Sciences 33(2-3):61-83; discussion: 83-135. doi: 10.1017/ S0140525X0999152X. [DA]

Henrich, J., McElreath, R., Barr, A., Ensminger, J., Barrett, C., Bolyanatz, A., Cardenas, J. C., Gurven, M., Gwako, E. L., Henrich, N. S., Lesorogol, C., Marlowe, F., Tracer, D. \& Ziker, J. (2006) Costly punishment across human societies. Science 312(5781):1767-70. doi: 10.1126/science.1127333. [arPR, SL, RM] 
References/Richerson et al.: Cultural group selection plays an essential role in explaining human cooperation

Henrich, N. \& Henrich, J. (2007) Why humans cooperate: A cultural and evolutionary explanation. Oxford University Press. [JH]

Henzi, S. P., de Sousa Pereira, L. F., Hawker-Bond, D., Stiller, J., Dunbar, R. I. M \& Barrett, L. (2007) Look who's talking: Developmental trends in the size of conversational cliques. Evolution and Human Behavior 28:66-74. [CA-C]

Herrmann, B., Thöni, C. \& Gächter, S. (2008) Antisocial punishment across societies. Science 319(5868):1362-67. doi: 10.1126/science.1153808. [arPR, DA, $\mathrm{PH}, \mathrm{SL}]$

Herrmann, E., Call, J., Hernandez-Lloreda, M. V., Hare, B. \& Tomasello, M. (2007) Humans have evolved specialized skills of social cognition: The cultural intelligence hypothesis. Science 317:1360-66. [AF]

Herrmann, P. A., Legare, C. H., Harris, P. L. \& Whitehouse, H. (2013) Stick to the script: The effect of witnessing multiple actors on children's imitation. Cognition 129(3):536-43. Available at: http://dx.doi.org/10.1016/j.cognition.2013.08. 010. [aPR]

Herzog, H. (2006) Forty-two thousand and one Dalmatians: Fads, social contagion, and dog breed popularity. Society and Animals 14(4):383-97. [HH]

Herzog, H. (2011) The impact of pets on human health and psychological well-being: Fact, fiction, or hypothesis? Current Directions in Psychological Science 20(4):236-39. [HH]

Herzog, H. A. (2014) Biology, culture, and the origins of pet-keeping. Animal Behavior and Cognition 1(3):296-308. [HH]

Herzog, H. A., Bentley, R. A. \& Hahn, M. W. (2004) Random drift and large shifts in popularity of dog breeds. Proceedings of the Royal Society B: Biological Sciences 271:353-56. [HH]

Hewlett, B., Fouts, H. N., Boyette, A. H. \& Hewlett, B. L. (2011) Social learning among Congo Basin hunter-gatherers. Philosophical Transactions of the Royal Society B 366:1168-78. [AW]

Hilbe, C. \& Traulsen, A. (2012) Emergence of responsible sanctions without second order free riders, antisocial punishment or spite. Scientific Reports 2, Article No. 458. Published Online, June 13, 2012. doi: 10.1036/srep00458. [MJG]

Hill, K., Barton, M. \& Hurtado, A. M. (2009) The emergence of human uniqueness: Characters underlying behavioral modernity. Evolutionary Anthropology 18:174-87. [aPR]

Hill, K. R., Walker, R. S., Božičević, M., Eder, J., Headland, T., Hewlett, B., Hurtado, A. M., Marlowe, F., Wiessner, P. \& Wood, B. (2011) Co-residence patterns in hunter-gatherer societies show unique human social structure. Science 331(6022):1286-89. [aPR]

Hill, K. R., Wood, B. M., Baggio, J., Hurtado, A. M. \& Boyd, R. T. (2014) Huntergatherer inter-band interaction rates: Implications for cumulative culture. PLoS ONE 9(7):e102806. doi: 10.1371/journal.pone.0102806. [arPR]

Hobson, P. (2004) The cradle of thought: Exploring the origins of thinking. Oxford University Press. [JSC]

Hodges, B. H. (2014) Rethinking conformity and imitation: divergence, convergence, and social understanding. Frontiers in Psychology: Cognitive Science 5, Article No. 726. doi: 10.3389/fpsyg.2014.00726. [SS]

Holden, C. J. (2002) Bantu language trees reflect the spread of farming across SubSaharan Africa: A maximum-parsimony analysis. Proceedings of the Royal Society: Biological Sciences 269(1493):793-99. doi: 10.2307/3067712. [aPR]

Holsinger, K. E. \& Weir, B. S. (2009) Genetics in geographically structured populations: Defining, estimating and interpreting $\mathrm{F}_{\mathrm{ST}}$. Nature Reviews Genetics 10 (9):639-50. doi: 10.1038/nrg2611. [RMR]

Horan, R. D., Bulte, E. \& Shoran, J. F. (2005) How trade saved humanity from biological exclusion: An economic theory of Neanderthal extinction. Journal of Economic Behavior and Organization 58:68-76. [CKWDD]

Horowitz, M. C. (2010) The diffusion of military power: Causes and consequences for international politics. Princeton University Press. [rPR]

Hout, M., Greeley, A. M. \& Wilde, M. J. (2001) The demographic imperative in religious change in the United States. American Journal of Sociology 107 (2):468-86. [aPR]

Howitt, A. W. (1885) The Jeraeil, or initiation ceremonies of the Kurnai tribe. Journal of the Anthropological Institute of Great Britain and Ireland 14:301-25. [MS]

Hrdy, S. B. (2009) Mothers and others: The evolutionary origins of mutual understanding. Harvard University Press. [aPR, JMB]

Hrdy, S. B. (2000) Mother nature: Maternal instincts and how they shape the human species. Ballantine. [HG]

Hugenberg, K. \& Bodenhausen, G. V. (2004) Ambiguity in social categorization: The role of prejudice and facial affect in racial categorization. Psychological Science $15: 342-45$. [aPR

Humle, T. \& Snowdon, C. T. (2008) Socially biased learning in the acquisition of a complex foraging task in juvenile cottontop tamarins (Saguinus oedipus). Animal Behaviour 27(1):267-77. [JMB]

Humle, T., Snowdon, C. T. \& Matsuzawa, T. (2009) Social influences on ant-dipping acquisition in the wild chimpanzees (Pan troglodytes verus) of Bossou, Guinea, West Africa. Animal Cognition 12(1):37-48. [JMB]

Hurford, J. R. (2007) The origins of meaning. Oxford University Press. [aPR]

Hurford, J. R. (2011) The origins of grammar: Language in the light of evolution II. Oxford University Press. [aPR]
Huxley, T. H. (1894) Discourses, biological and geological: Essays (vol. 8). D. Appleton. [SL]

Hwang, V. W. \& Horowitt, G. (2012) The rainforest: The secret to building the next Silicon Valley. Regewald. [aPR]

Iannaccone, L. R. (1992) Sacrifice and stigma: Reducing free-riding in cults, communes, and other collectives. The Journal of Political Economy 100 (2):271-91. [aPR]

Iannaccone, L. R. (1994) Why strict churches are strong. American Journal of Sociology 99(5):1180-1211. [aPR]

Iannaccone, L. R. (1998) Introduction to the economics of religion. Journal of Economic Literature 36(2):1465-95. [PH]

Ibrahim, M. (1990) Merchant capital and Islam. University of Texas Press. [aPR]

Insko, C. A., Gilmore, R., Drenan, S., Lipsitz, A., Moehle, D. \& Thibaut, J. (1983) Trade versus expropriation in open groups: A comparison of two type of social power. Journal of Personality and Social Psychology 44:977-99. [arPR]

Itan, Y., Powell, A., Beaumont, M. A., Burger, J. \& Thomas, M. G. (2009) The origins of lactase persistence in Europe. PLoS Computational Biology 5(8):e1000491. doi: 10.1371/journal.pcbi.1000491. [arPR]

Jablonka, E. \& Lamb, M. (2005) Evolution in four dimensions: Genetic, epigenetic, behavioral, and symbolic variation in the history of life. MIT Press. [AF]

Jacobs, R. C. \& Campbell, D. T. (1961) The perpetuation of an arbitrary tradition through several generations of laboratory microculture. Journal of Abnormal and Social Psychology 62:649-58. [aPR]

Jaswal, V. K. (2010) Believing what you're told: Young children's trust in unexpected testimony about the physical world. Cognitive Psychology 61(3):248-72. [SS]

Jaswal, V. K., Croft, A. C., Setia, A. R. \& Cole, C. A. (2010) Young children have a specific, highly robust bias to trust testimony. Psychological Science 21 (10):1541-47. [SS]

Jaswal, V. K. \& Neely, L. A. (2006) Adults don’t always know best: Preschoolers use past reliability over age when learning new words. Psychological Science 17:757-58. [SS]

Jobling, M. A. (2012) The impact of recent events on human genetic diversity. Philosophical Transactions of the Royal Society B: Biological Sciences 367 (1590):793-99. doi: 10.1098/rstb.2011.0297. [RMR]

John, O. P., Naumann, L. P. \& Soto, C. J. (2008) Paradigm shift to the integrative big five trait taxonomy. Handbook of personality: Theory and research 3:114-58. [MJG]

Johnson, A. W. \& Earle, T. (2000) The evolution of human societies: From foraging group to agrarian state, 2 nd edition. Stanford University Press. [PT]

Johnson, D. (2005) God's punishment and public goods. Human Nature 16 (4):410-46. [aPR]

Jones, D. M., Bremer, S. A. \& Singer, J. D. (1996) Militarized interstate disputes, 1816-1992: Rationale, coding rules, and empirical patterns. Conflict Management and Peace Science 15:163-213. [CKWDD]

Jordan, P. (2015) Technology as human social tradition: Cultural transmission among hunter-gatherers. University of California Press. [rPR, AW]

Jorgensen, J. G. (1980) Western Indians: Comparative environments, languages, and cultures of 172 Western American Indian tribes. W. H. Freeman. [aPR]

Jurmain, R., Nelson, H., Kilgore, L. \& Travathan, W. (1997) Introduction to physical anthropology. Wadsworth. [HG]

Keeley, L. H. (1997) War before civilization. Oxford University Press. [aPR]

Kelly, R. C. (1980) Etoro social structure: A study in structural contradiction. The University of Michigan Press. [MS]

Kelly, R. C. (1985) The Nuer conquest: The structure and development of an expansionist system. University of Michigan Press. [arPR]

Kempe, M. \& Mesoudi, A. (2014) Experimental and theoretical models of human cultural evolution. Wiley Interdisciplinary Reviews: Cognitive Science 5(3):31726. doi: 10.1002/wcs.1288. [aPR]

Kinzler, K. D., Corriveau, K. H. \& Harris, P. L. (2011) Children's selective trust in native-accented speakers. Developmental Science 14:106-111. [aPR]

Kinzler, K. D., Shutts, K., DeJesus, J. \& Spelke, E. S. (2009) Accent trumps race in guiding children's social preferences. Social Cognition 27(4):623-34. doi: 10.1521/soco.2009.27.4.623 [aPR]

Kirschner, S. \& Tomasello, M. (2010) Joint music making promotes prosocial behavior in 4-year-old children. Evolution and Human Behavior 31(5):354-64 doi: 10.1016/j.evolhumbehav.2010.04.004. [aPR]

Klepper, S. \& Sleeper, S. (2005) Entry by spinoffs. Management Science 51(8):12911306. [aPR]

Kline, M. A. (2015) How to learn about teaching: An evolutionary framework for the study of teaching in humans and other animals. Behavioral and Brain Sciences 38:e31. Available at: dx.doi.org/10.1017/S0140525X14000090 [aPR, JMB]

Knauft, B. M. (1985) Good company and violence: Sorcery and social action in a lowland New Guinea Society. University of California Press. [rPR]

Knobel, D. L. (2009) Aspects of dog ownership and canine rabies control in Africa and Asia. Doctoral dissertation, Centre for Tropical Veterinary Medicine, Roya (Dick) School of Veterinary Studies, University of Edinburgh. [HH]

Koenig, M. A. \& Echols, C. H. (2003) Infants' understanding of false labeling events: The referential roles of words and the speakers who use them. Cognition 87 (3): 179-208. [SS] 
Koenig, M. A. \& Harris, P. L. (2005) Preschoolers mistrust ignorant and inaccurate speakers. Child Development 76(6):1261-77. [SS]

Koenig, M. A. \& Jaswal, V. K. (2011) Characterizing children's expectations about expertise and incompetence: Halo or pitchfork effects? Child Development 82 (5):1634-47. [SS]

Koenig, M. A. \& Stephens, E., (2014) Characterizing children's responsiveness to cues of speaker trustworthiness: Two proposals. In: Trust and skepticism: Children's selective learning from testimony, ed. E. Robinson \& S. Einav, pp. 13-27. Psychology Press. [SS]

Koenig, M. A. \& Woodward, A. L. (2010) Sensitivity of 24-month-olds to the prior inaccuracy of the source: Possible mechanisms. Developmental Psychology 46 (4):815-26. [SS]

Kohler, H.-P., Rogers, J. L. \& Christensen, K. (1999) Is fertility behavior in our genes? Findings from a Danish twin study. Population and Development Review 25:253-88. [aPR]

Kosfeld, M., Heinrichs, M., Zak, P. J., Fischbacher, U. \& Fehr, E. (2005) Oxytocin increases trust in humans. Nature 435:673-76. [JSC]

Koster, J. M. (2009) Hunting dogs in the lowland Neotropics. Journal of Anthropological Research 65:575-610. [HH]

Kovarik, J. (2009) Giving it now or later: Altruism and discounting. Economics Letters 102(3):152-54. [DA]

Kramer, K. L. (2010) Cooperative breeding and its significance to the demographic success of humans. Annual Review of Anthropology 39:417-36. [JMB]

Krasnow, M. M., Cosmides, L., Pedersen, E. J. \& Tooby, J. (2012) What are punishment and reputation for? PLoS ONE 7(9):e45662. doi: 10.1371/journal. pone.0045662. [aPR, MMK, JT]

Kraybill, D. B. \& Bowman, C. F. (2001) On the backroad to heaven: Old Order Hutterites, Mennonites, Amish, and Brethren. Johns Hopkins University Press. [aPR]

Krebs, J. R. \& Davies, N. B. (1993) An introduction to behavioural ecology, 3rd edition. Blackwell. [MMK]

Kuběna, A. A., Houdek, P., Lindová, J., Příplatová, L. \& Flegr, J. (2014) Justine effect: Punishment of the unduly self-sacrificing cooperative individuals. PLoS One 9(3):e92336. doi: 10.1371/journal.pone.0092336. [PH]

Kurzban, R. \& Houser, D. (2005) Experiments investigating cooperative types in humans: A complement to evolutionary theory and simulations. Proceedings of the National Academy of Sciences USA 102(5):1803-1807. doi: 10.1073/ pnas.0408759102. [aPR]

Labov, W. (2001) Principles of linguistic change: Social factors, vol. 29. Blackwell. $\quad$ [aPR]

Lachmann, M., Szamado, S. \& Bergstrom, C. T. (2001) Cost and conflict in animal signals and human language. Proceedings of the National Academy of Sciences USA 98(23):13189-94. [aPR]

Lakatos, I. (1978) The methodology of scientific research programmes. Cambridge University Press. [PT]

Laland, K. N., Odling-Smee, J. \& Myles, S. (2010) How culture shaped the human genome: Bringing genetics and the human sciences together. Nature Reviews Genetics 11(2):137-48. [arPR]

Laland, K. N., Sterelny, K., Odling-Smee, J., Hoppitt, W. \& Uller, T. (2011) Cause and effect in biology revisited: Is Mayr's proximate-ultimate dichotomy still useful? Science 334(6062):1512-16. doi: 10.1126/ science.1210879. [arPR]

Laland, K. N., Tobias, U., Feldman, M., Sterelny, K., Muller, G., Moczek, A., Jabonka, E. \& Odling-Smee, J. (2014) Does evolutionary theory need a rethink? Yes, urgently. Nature 514:161-64. [AF]

Laland, K. N., Uller, T., Feldman, M. W., Sterelny, K., Müller, G. B., Moczek, A., Jablonka, E. \& Odling-Smee, J. (2015) The extended evolutionary synthesis: Its structure, assumptions and predictions Proceedings of the Royal Society of London B 282(1813):20151019. http://dx.doi.org/10.1098/rspb.2015.1019. [AF]

Lamba, S. (2014) Social learning in cooperative dilemmas. Proceedings of the Royal Society of London B: Biological Sciences 281(1787):e20140417. (Published Online May 28, 2014). Available at: http://dx.doi.org/10.1098/rspb.2014.0417. [SL, rPR]

Lamba, S. \& Mace, R. (2011) Demography and ecology drive variation in cooperation across human populations. Proceedings of the National Academy of Sciences USA 108(35):14426-30. doi: 10.1073/pnas.1105186108. [JH, SL, $\mathrm{RM}, \mathrm{rPR}]$

Lamba, S. \& Mace, R. (2012) Reply to Henrich et al.: Behavioral variation needs to be quantified at multiple levels. Proceedings of the National Academy of Sciences USA 109(2):e34. doi: 10.1073/pnas.1118858109. Available at: http://www pnas.org/content/109/2/E34.full. [SL, RM]

Lamba, S. \& Mace, R. (2013) The evolution of fairness: Explaining variation in bargaining behaviour. Proceedings of the Royal Society B: Biological Sciences 280(1750):e201220128. (Published Online November 21, 2012). doi: 10.1098/ rspb.2012.2028. Available at: http://rspb.royalsocietypublishing.org/content/ 280/1750/20122028. [SL]

Lane, A., Luminet, O., Rimé, B., Gross, J. J., de Timary, P. \& Mikolajczak, M. (2013) Oxytocin increases willingness to socially share one's emotions. International Journal of Psychology 48(4):676-81. [JSC]
Lane, D., Maxfield, R. M., Read, D. \& van der Leeuw, S. (2009) From population to organization thinking. In: Complexity perspective in innovation and social change, ed. D. Lane, D. Pumain, S. E. van der Leeuw \& G. West, pp. 11-42. Springer. [DR]

Lao, O., Lu, T. T., Nothnagel, M., Junge, O., Freitag-Wolf, S., Caliebe, A., Balascakova, M., Bertranpetit, J., Bindoff, L. A., Comas, D., Holmlund, G., Kouvatsi, A., Macek, M., Mollet, I., Parson, W., Palo, J., Ploski, R., Sajantila, A., Tagliabracci, A., Gether, U., Werge, T., Rivadeneira, F., Hofman, A., Uitterlinden, A. G., Gieger, C., Wichmann, H. E., Rüther, A., Schreiber, S., Becker, C., Nümberg, P., Nelson, M. R., Krawczak, M. \& Kayser, M. (2008) Correlation between genetic and geographic structure in Europe. Current Biology 18 (16):1241-48. doi: 10.1016/j.cub.2008.07.049. [RMR]

Leaf, M. \& Read, D. (2012) Human thought and social organization: Anthropology on a new plane. Lexington Press. [DR]

Lee, R. B. (1972) !Kung spatial organization: An ecological and historical perspective. Human Ecology 1(2):125-47. doi: 10.2307/4602251. [aPR]

Lehmann, L., Feldman, M. W. \& Foster, K. (2008) Cultural transmission can inhibit the evolution of altruistic helping. The American Naturalist 172:12-24. [aPR]

Levieil, D. P. \& Orlove, B. (1990) Local control of aquatic resources: Community and ecology in Lake Titicaca, Peru. American Anthropologist 92(2):362-82. doi: $10.2307 / 680150$. [rPR]

Levy, G. D., Taylor, M. G. \& Gelman, S. A. (1995) Traditional and evaluative aspects of flexibility in gender roles, social conventions, moral rules, and physical laws. Child Development 66(2):515-31. [aPR]

Li, H. \& Durbin, R. (2011) Inference of human population history from individual whole-genome sequences. Nature 475(7357):493-96. doi: 10.1038/ nature10231. Available at: http://www.nature.com/nature/journal/v475/n7357/ full/nature10231.html. [rPR]

List, J. (2007) On the interpretation of giving in dictator games. Journal of Political Economy 115(3):482-93. [JT, rPR]

Llewellyn, K. N. \& Hoebel, E. A. (1941) The Cheyenne way: Conflict and case law in primitive jurisprudence. University of Oklahoma Press. [MS]

Lonsdorf, E. (2006) What is the role of mothers in the acquisition of termite-fishing behaviors in wild chimpanzees (Pan troglodytes schweinfurthii)? Animal Cognition 9(1):36-46. [JMB]

Lotz, S. (2014) Spontaneous giving under structural inequality: Intuition promotes cooperation in asymmetric social dilemmas. Available at Social Science Research Network (SSRN): http://ssrn.com/abstract=2513498. [DA]

Lyon, B. E. \& Montgomerie, R. (2012) Sexual selection is a form of social selection. Philosophical Transactions of the Royal Society of London. Series B: Biological Sciences 367(1600):2266-73. doi: 10.1098/rstb.2012.0012. [RMN]

Macdonald, G. \& Leary, M. R. (2005) Why does social exclusion hurt? The relationship between social and physical pain. Psychological Bulletin 131(2):202-23. doi:10.1037/0033-2909.131.2.202. [JMV]

Mace, R. \& Jordan, F. M. (2011) Macro-evolutionary studies of cultural diversity: A review of empirical studies of cultural transmission and cultural adaptation. Philosophical Transactions of the Royal Society B: Biological Sciences 366 (1563):402-11. [aPR]

MacKinnon, K. C. \& Fuentes, A. (2011) Primates, niche construction, and social complexity: The roles of social cooperation and altruism. In: Origins of altruism and cooperation. Developments in primatology: Progress and prospects, vol. 36 Part 2, ed. R. W. Sussman \& R. C. Cloninger, pp. 121-43. Springer. [AF]

Madsen, W. (1967) Religious syncretism. In: Handbook of Middle American Indians, vol. 6, ed. R. Wauchope, pp. 369-91. University of Texas Press. [CSa]

Malinowski, B. (1926) Crime and custom in savage society. Harcourt, Brace. [MS]

Malone, N. M., Fuentes, A. \& White, F. J. (2012) Variation in the social systems of extant hominoids: Comparative insight into the social behaviour of early hominins. International Journal of Primatology 33(6):1251-77. doi: 10.10007/ s10764-012-9617-0. [AF]

Mania, D. \& Mania, U. (1988) Deliberate engravings on bone artefacts of Homo erectus. Rock Art Research 5:91-107. [AF]

Marean, C. W. (2010) Pinnacle Point Cave 13B (Western Cape Province, South Africa) in context: The Cape Floral Kingdom, shellfish, and modern human origins. Journal of Human Evolution 59(3-4):425-43. doi: 10.1016/j. jhevol.2010.07.011. [aPR]

Markus, H. R. \& Kitayama, S. (1991) Culture and the self: Implications for cognition, emotion, and motivation. Psychological Review 98:224-53. [PB]

Marshall, J. A. R. (2011) Group selection and kin selection: Formally equivalent approaches. Trends in Ecology and Evolution 26:325-32. doi:10.1016/j. tree.2011.04.008. [PB]

Martynova, M. \& Renneboog, L. (2008) A century of corporate takeovers: What have we learned and where do we stand? Journal of Banking and Finance 32:214877. [CKWDD]

Mäs, M. \& Dijkstra, J. (2014) Do intergroup conflicts necessarily result from outgroup hate? PLoS One 9(6):e97848. [CA-C]

Mathew, S. \& Boyd, R. (2011) Punishment sustains large-scale cooperation in prestate warfare. Proceedings of the National Academy of Sciences USA 108 (28):11375-80. doi: 10.1073/pnas.1105604108. [aPR] 
References/Richerson et al.: Cultural group selection plays an essential role in explaining human cooperation

Mathew, S., Boyd, R. \& Van Veelen, M. (2013) Human cooperation among kin and close associates may require enforcement of norms by third parties. In: Cultural evolution, ed. P. J. Richerson \& M. Christiansen, pp. 45-60. MIT Press. [arPR, JH]

Maynard Smith, J. \& Szathmáry, E. (1995) The major transitions in evolution. W. H. Freeman/Spectrum. [arPR, PT]

McCullough, M. E., Pedersen, E. J., Schroder, J. M., Tabak, B. A. \& Carver, C. S. (2013) Harsh childhood environmental characteristics predict exploitation and retaliation in humans. Proceedings of the Royal Society B: Biological Sciences 280(1750): 20122104. doi: 10.1098/rspb.2012.2104. [JH]

McDowell, A. (2004) Real property, spontaneous order, and norms in the gold mines. Law and Social Inquiry 29(4):771-818. Available at: http://onlinelibrary. wiley.com/doi/10.1111/j.1747-4469.2004.tb01076.x/abstract. [MS]

McElreath, R. (2004) Social learning and the maintenance of cultural variation: An evolutionary model and data from East Africa. American Anthropologist 106 (2):308-21. doi: 10.1525/aa.2004.106.2.308. [JH]

McElreath, R., Bell, A. V., Efferson, C., Lubell, M., Richerson, P. J. \& Waring, T (2008) Beyond existence and aiming outside the laboratory: Estimating frequency-dependent and pay-off-biased social learning strategies. Philosophical Transactions of the Royal Society B 363:3515-28. [rPR]

McElreath, R., Boyd, R. \& Richerson, P. (2003) Shared norms can lead to the evolution of ethnic markers. Current Anthropology 44(1):123-29. [aPR]

McGovern, T. (2007) Why do successful companies fail? A case study of the decline of Dunlop. Business History 49:886-907. doi: 10.1080/00076790701710407. [aPR]

McKay, R. T., Efferson, C., Whitehouse, H. \& Fehr, E. (2011) Wrath of God: Religious primes and punishment. Proceedings of the Royal Society B 278 (1713):1858-63. (Epub November 24, 2010). doi: 10.1098/rspb.2010.2125. [aPR]

McNeill, W. H. (1995) Keeping together in time: Dance and drill in human history. Harvard University Press. [aPR]

Meins, E., Fernyhough, C., Wainwright, R., Das Gupta, M., Fradley, E. \& Tuckey, M. (2002) Maternal mind-mindedness and attachment security as predictors of theory of mind understanding. Child Development 73(6):1715-26. [JSC]

Mercier, H. \& Sperber, D. (2011) Why do humans reason? Arguments for an argumentative theory. Behavioral and Brain Sciences 34(2):57-74. [SS]

Moffett, M. W. (2013) Human identity and the evolution of societies. Human Nature 24(3):219-67. doi: 10.1007/s12110-013-9170-3. [aPR]

Molleman, L., van den Berg, P. \& Weissing, F. J. (2014) Consistent individual differences in human social learning strategies. Nature Communications 5:1-9, Article No. 3570. doi: 10.1038/ncomms4570. (Online publication) Available at: http://www.nature.com/ncomms/2014/140404/ncomms4570/full/ncomms4570. html. [aPR]

Morin, O. (2014) Is cooperation a maladaptive by-product of social learning? The docility hypothesis reconsidered. Biological Theory 9(3):286-95. [OM]

Motta, M. (2004) Competition policy: Theory and practice. Cambridge University Press. [aPR]

Mrazek, A. J., Chiao, J. Y., Blizinsky, K. D., Lun, J. \& Gelfand, M. J. (2013) The role of culture-gene coevolution in morality judgment: Examining the interplay between tightness-looseness and allelic variation of the serotonin transporter gene. Culture and Brain 1(2-4):100-117. [MJG]

Mu, Y., Kitayama, S., Han, S., \& Gelfand, M. J. (2015) How culture gets embrained: Cultural differences in event-related potentials of social norm violations. Proceedings of the National Academy of Sciences USA 112(50):15348-53. doi: 10.1073/pnas.1509839112. [MJG, rPR]

Müller, M. (1862) Lectures on the science of language, 3rd edition. Longman, Green, Longman and Roberts. [aPR]

Murmann, J. P. (2003) Knowledge and competitive advantage: The coevolution of firms, technology, and National institutions. Cambridge University Press. [aPR]

Murphy, R. F. \& Murphy, Y. (1986) Northern Shoshone and Bannock. In: Handbook of North American Indians: Great Basin, vol. 11, ed. W. L. d'Azevedo, pp. 284307. Smithsonian Institution Press. [aPR]

Nader, L. (1969) Law in culture and society. Aldine. [aPR]

Nelson, E. E. \& Panksepp, J. (1998) Brain substrates of infant-mother attachment: Contributions of opioids, oxytocin, and norepinephrine. Neuroscience and Biobehavioral Reviews 22(3):437-52. [JSC]

Nelson, R. R. \& Winter, S. G. (1982) An evolutionary theory of economic change. Belknap Press of Harvard University Press. [aPR]

Nesse, R. M. (2009) Runaway social selection for displays of partner value and altruism. In: The moral brain: Essays on the evolutionary and neuroscientific aspects of morality, ed. J. Verplaetse, J. De Schrijver, S. Vanneste \& J. Braeckman, pp. 211-32. Springer-Verlag. [RMN]

Nesse, R. M. (2010) Social selection and the origins of culture. In: Evolution, culture and the human mind, ed. M. Schaller, S. J. Heine, A. Norenzayan, T. Yamagishi \& T. Kameda, pp. 137-50. Psychology Press. [RMN]

Nettle, D. (1999) Linguistic diversity. Oxford University Press. [aPR]

Nettle, D., Colléony, A. \& Cockerill, M. (2011) Variation in cooperative behaviour within a single city. PLoS One 6(10):e26922. [SL, RM]
Newmeyer, F. J. (2004) Against a parameter-setting approach to typological variation. Linguistic Variation Yearbook 4(1):181-34. doi: 10.1075/livy.4.06new. [aPR]

Newson, L. (2013) Cultural evolution and human reproductive behavior. In: Building babies: Primate development in proximate and ultimate perspective, ed. K. B. H. Clancy, K. Hinde \& J. N. Rutherford, pp. 481-503. Springer Science. [aPR]

Nisbett, R. E. \& Cohen, D. (1996) Culture of Honor: The psychology of violence in the South. Westview Press. [rPR, PB]

Nivette, A. E. (2011) Cross-national predictors of crime: A meta-analysis. Homicide Studies 15(2):103-31. doi: 10.1177/1088767911406397. [aPR]

Noë, R., van Hooff, J. A. R. A. M. \& Hammerstein, P. (2001) Economics in nature Social dilemmas, mate choice and biological markets. Cambridge University Press. [RMN]

Norenzayan, A. \& Shariff, A. F. (2008) The origin and evolution of religious prosociality. Science 322(5898):58-62. doi: 10.1126/science.1158757 [aPR]

Norenzayan, A., Shariff, A. F., Gervais, W. M., Willard, A. K., McNamara, R. A., Slingerland, E. \& Henrich, J. (2016) The cultural evolution of prosocial religions. Behavioral and Brain Sciences 39. doi: 10.1017/S0140525X14001356. [PH, MS]

North, D. C., Wallis, J. J. \& Weingast, B. R. (2009) Violence and social orders: A conceptual framework for interpreting recorded human history. Cambridge University Press. [arPR]

Novembre, J., Johnson, T., Bryc, K., Kutalik, Z., Boyko, A. R., Auton, A., Indap, A., King, K. S., Bergmann, S., Nelson, M. R., Stephens, M. \& Bustamante, C. D. (2008) Genes mirror geography within Europe. Nature 456(7218):98-101. doi 10.1038/nature07331. [RMR]

Okasha, S. (2015) The relation between kin and multi-level selection: An approach using causal graphs. British Journal for the Philosophy of Science. doi: 10.1093/ bjps/axu047. [PB]

Okasha, S. \& Paternotte, C. (2012) Group adaptation, formal Darwinism and contextual analysis. Journal of Evolutionary Biology 25:1127-39. [PB]

Olsson, O. \& Paik, C. (2013) A western reversal since the Neolithic? The long-run impact of early agriculture. University of Gothenburg Working Papers in Economics. [PH]

Ostrom, E. (1990) Governing the Commons: The evolution of institutions for collective action. Cambridge University Press. [aPR, MS]

Ostrom, E. (2005) Understanding institutional diversity. Princeton University Press. [arPR]

Paciotti, B., Hadley, C., Holmes, C. \& Borgerhoff Mulder, M. (2005) Grass-roots justice in Tanzania. American Scientist 93:58-65. [aPR]

Panchanathan, K. \& Boyd, R. (2004) Indirect reciprocity can stabilize cooperation without the second-order free rider problem. Nature 432:499-502. $[\mathrm{aPR}, \mathrm{JH}]$

Panksepp, J. (1998) Affective neuroscience: The foundations of human and animal emotions. Oxford university press. [JMV]

Panksepp, J. (2011) The basic emotional circuits of mammalian brains: Do animals have affective lives? Neuroscience and Biobehavioral Reviews 35:1791-804. [JSC]

Panksepp, J. \& Biven, L. (2012) The archaeology of mind: Neuroevolutionary origins of human emotions. W. W. Norton. [rPR]

Pedersen, E. J., Kurzban, R. \& McCullough, M. E. (2013) Do humans really punish altruistically? A closer look. Proceedings of the Royal Society B: Biological Sciences 280(1758):e20122723. doi: 10.1098/rspb.2012.2723. Online paper available at: http://rspb.royalsocietypublishing.org/content/280/1758/20122723. [JT]

Peer, P. Van, Rots, V. \& Vroomans, J. (2004) A story of colourful diggers and grinders: The Sangoan and Lupemban at site 8-B-11, Sai Island, Northern Sudan. Before Farming 3:1-28. [AF]

Perreault, C. (2012) The pace of cultural evolution. PLoS ONE 7(9):e45150. doi: 10.1371/journal.pone.0045150. [aPR]

Petersen, M. B., Sznycer, D., Cosmides, L. \& Tooby, J. (2012) Who deserves help? Evolutionary psychology, social emotions, and public opinion about welfare. Political Psychology 33(3):395-18. [JT]

Petersen, M. B., Sznycer, D., Sell, A., Cosmides, L. \& Tooby, J. (2013) The ancestral logic of politics: Upper-body strength regulates men's assertion of self-interest over economic redistribution. Psychological Science 24(7):1098-103. [JT]

Peysakhovich, A. \& Rand, D. G. (2015) Habits of virtue: Creating norms of cooperation and defection in the laboratory. Management Science. doi: 10.1287/ mnsc.2015.2168 [DA]

Pickrell, J. K. \& Reich, D. (2014) Toward a new history and geography of human genes informed by ancient DNA. Trends in Genetics 30(9):377-89. Available at: http://dx.doi.org/10.1016/j.tig.2014.07.007. [rPR]

Pinker, S. (1994) The language instinct: How the mind creates language. Morrow. [aPR]

Pinker, S. (2010) The cognitive niche: Coevolution of intelligence, sociality, and language. Proceedings of the National Academy of Sciences USA 107(Suppl. 2):8993-99. doi: 10.1073/pnas.0914630107. [aPR, JT]

Pinker, S. (2012) The false allure of group selection. An Edge original essay. Available at: http://edge.org/conversation/the-false-allure-of-group-selection. [aPR, MMK, JH] 
Pinker, S. \& Bloom, P. (1990) Natural language and natural selection. Behavioral and Brain Sciences 13:707-84. [aPR]

Preston, S. D. \& De Waal, F. (2002) Empathy: Its ultimate and proximate bases. Behavioral and Brain Sciences 25(1):1-20. [HH]

Price, G. R. (1970) Selection and covariance. Nature 277:520-21. [rPR]

Price, G. R. (1972) Extensions of covariance selection mathematics. Annals of Human Genetics 35:485-90. [rPR, PB]

Prietula, M. \& Conway, D. (2009) The evolution of metanorms: Quis custodiet ipsos custodes? Computational and Mathematical Organization Theory 15(3):14768. doi: 10.1007/s10588-009-9056-4. [PH]

Pritchard, J. K., Pickrell, J. K. \& Coop, G. (2010) The genetics of human adaptation: Hard sweeps, soft sweeps, and polygenic adaptation. Current Biology 20(4): R208-R215. doi: 10.1016/j.cub.2009.11.055. [aPR]

Pruitt, D. G. \& Rubin, J. Z. (1986) Social conflict: Escalation, stalemate, and settlement. McGraw Hill. [CKWDD]

Putnam, R. (1993) Making democracy work. Princeton University Press. [CA-C]

Queller, D. C. (1992) Quantitative genetics, inclusive fitness, and group selection. American Naturalist 139:540-58. [PB]

Rachlin, H. (2002) Altruism and selfishness. Behavioral and Brain Sciences 25:23996. [CSi]

Rachlin, H. (2004) The science of self-control. Harvard University Press. [CSi]

Rand, D. G., Greene, J. D. \& Nowak, M. A. (2012) Spontaneous giving and calculated greed. Nature 489(7416):427-30. [DA]

Rand, D. G. \& Kraft-Todd, G. T. (2014) Reflection does not undermine self-interested prosociality. Frontiers in Behavioral Neuroscience 8:300. [DA]

Rand, D. G., Newman, G. E. \& Wurzbacher, O. (2014a) Social context and the dynamics of cooperative choice. Journal of Behavioral Decision Making. doi: 10.1002/bdm.1837. [DA]

Rand, D. G. \& Nowak, M. A. (2013) Human cooperation. Trends in Cognitive Sciences 17(8):413-25. [DA]

Rand, D. G., Peysakhovich, A., Kraft-Todd, G. T., Newman, G. E., Wurzbacher, O., Nowak, M. A. \& Green, J. D. (2014b) Social heuristics shape intuitive cooperation. Nature Communications 5:3677. [DA]

Rapaport, L. G. (2011) Progressive parenting behavior in wild golden lion tamarins. Behavioral Ecology 22(4):745-54. [JMB]

Rappaport, R. (1999) Ritual and religion in the making of humanity. Cambridge University Press. [aPR]

Read, D. (1984) An algebraic account of the American kinship terminology. Current Anthropology 25:417-40. [DR]

Read, D. (2005) Some observations on resilience and robustness in human systems. Cybernetics and Systems (Special Issue) 36:773-802. [DR]

Read, D. (2012) How culture makes us human. [Big Ideas in Little Books series]. Left Coast Press. [DR]

Read, D. (2013) Reconstructing the Proto-Polynesian terminology: Kinship terminologies as evolving logical structures. In: Kinship systems: Change and reconstruction, ed. P. McConvell, I. Keen \& R. Hendery, pp. 59-91. University of Utah Press. [DR]

Read, D., Fischer, M. D. \& Chit Hlaing, F. K. L. (2014) The cultural grounding of kinship: A paradigm shift. L'Homme 210(2):63-89. [DR]

Reeve, H. K. (1992) Queen activation of lazy workers in colonies of the eusocial naked mole-rat. Nature 358:147-49. [MS]

Reifen Tagar, M., Federico, C. M., Lyons, K. E., Ludeke, S. \& Koenig, M. A. (2014) Heralding the authoritarian? Orientation toward authority in early childhood. Psychological Science 25(4):883-92. [SS]

Reiter, D. \& Stam, A. C. (2002) Democracies at war: Princeton University Press. [aPR]

Relethford, J. H. (2007) The human species: An introduction to biological anthropology. McGraw-Hill. [HG]

Richerson, P. J. \& Boyd, R. (1998) The evolution of human ultrasociality. In: Indoctrinability, ideology, and warfare: Evolutionary perspectives, ed. I. EiblEibesfeldt \& F. K. Salter, pp. 71-95. Berghahn Books. [aPR]

Richerson, P. J. \& Boyd, R. (1999) Complex societies: The evolutionary origins of a crude superorganism. Human Nature 10(3):253-89. [aPR, JH]

Richerson, P. J. \& Boyd, R. (2005) Not by genes alone: How culture transformed human evolution. University of Chicago Press. [PB]

Richerson, P. J. \& Boyd, R. (2010) The Darwinian theory of cultural evolution and gene-culture coevolution. In: Evolution since Darwin: The first 150 years, ed. M. A. Bell, D. J. Futuyma, W. F. Eanes \& J. S. Levinton, pp. 561-88. Sinauer. [aPR]

Richerson, P. J., Boyd, R. \& Henrich, J. (2010) Gene-culture coevolution in the age of genomics. Proceedings of the National Academy of Sciences USA 107(Suppl. 2):8985-92. [arPR]

Roach, N. T., Venkadesan, M., Rainbow, M. J. \& Lieberman, D. E. (2013) Elastic energy storage in the shoulder and the evolution of high-speed throwing in Homo. Nature 498(7455):483-86. [HG]

Roberts, S. G. B., Dunbar, R. I. M., Pollet, T. V. \& Kuppens, T. (2009) Exploring variation in active network size: Constraints and ego characteristics. Social Networks 31:138-46. [CA-C]
Roch, S. G., Lane, J. A. S., Samuelson, C. D., Allison, S. T. \& Dent, J. L. (2000) Cognitive load and the equality heuristic: A two-stage model of resource overconsumption in small groups. Organizational Behavior and Human Decision Processes 83(2):185-212. doi: 10.1006/obhd.2000.2915. [DA]

Rockenbach, B. \& Milinski, M. (2006) The efficient interaction of indirect reciprocity and costly punishment. Nature 444(7120):718-23. [DA]

Roebroeks, W., Sier, M. J., Nielsen, T. K., De Loecker, D., Parés, J. M., Arps, C. E. S. \& Mücher, H. J. (2012) Use of red ochre by early Neandertals. Proceedings of the National Academy of Sciences USA 109(6):1889-94. [AF]

Rogers, A. R. (1990) Group selection by selective emigration. The American Naturalist 135(3):398-13. [rPR]

Rogers, E. M. \& Shoemaker, F. F. (1971) Communication of innovations: A crosscultural approach, 2 nd edition. Free Press. [aPR]

Roos, P., Gelfand, M., Nau, D. \& Carr, R. (2014) High strength-of-ties and low mobility enable the evolution of third-party punishment. Proceedings of the Royal Society B: Biological Sciences 281(1776): Article no. 2661. doi: 10.1098/ rspb.2013.2661. [MJG]

Roos, P., Gelfand, M. J., Nau, D. \& Lun, J. (2015) Societal threat and cultural variation in the strength of social norms: An evolutionary basis. Organizational Behavior and Human Decision Processes 129:14-23. [MJG]

Ross, C. T. \& Richerson, P. J. (2014) New frontiers in the study of cultural and genetic evolution. Current Opinion in Genetics and Development 29:103109. [aPR]

Ross, R. M., Greenhill, S. J. \& Atkinson, Q. D. (2013) Population structure and cultural geography of a folktale in Europe. Proceedings of the Royal Society B: Biological Sciences 280(1756):20123065. doi: 10.1098/rspb.2012.3065. [RMR]

Roy, S., Miller, C. T., Gottsch, D. \& Wang, X. (2011) Vocal control by the common marmoset in the presence of interfering noise. The Journal of Experimental Biology 214(21):3619-29. [JMB]

Rukstalis, M., Fite, J. E. \& French, J. A. (2003) Social change affects vocal structure in a callitrichid primate (Callithrix kuhlii). Ethology 109:327-40. [JMB]

Runciman, W. G. (2005) Stone Age sociology. Journal of the Royal Anthropological Institute 11(1):129-42. doi: 10.1111/j.1467-9655.2005.00229.x. [aPR]

Rushton, J. P. (1989) Genetic similarity, human altruism, and group selection. Behavioral and Brain Sciences 12(3):503-18. doi:10.1017/ S0140525X00057320. [aPR]

Sabbagh, M. A. \& Shafman, D. (2009) How children block learning from ignorant speakers. Cognition 112:415-22. [SS]

Salali, G. D., Juda, M. \& Henrich, J. (2015) Transmission and development of costly punishment in children. Evolution and Human Behavior 36(2):86-94. doi: 10.1016/j.evolhumbehav.2014.09.004. [JH]

Sampson, G. (2005) The "Language instinct" debate, revised edition. Continuum. [aPR]

Sato, T., Nonaka, K., Miura, T. \& Peter, K. (1994) Trends in cohort fertility of the Dariusleut Hutterite population. Human Biology 66(3):421-32. [aPR]

Schapera, I. (1970) Tribal innovators: Tswana chiefs and social change, 1795-1940. The Athlone Press. [MS]

Schelling, T. (1960) The strategy of conflict. Harvard University Press. [CKWDD]

Schore, A. (2013) Bowlby's "environment of evolutionary adaptedness": Recent studies on the interpersonal neurobiology of attachment and emotional development. In: Evolution, early experience and human development, ed. D. Narvaez, J. Panksepp, A. Schore \& T. Gleason, pp. 31-67. Oxford University Press. [JSC]

Schulz, J. F., Fischbacher, U., Thöni, C. \& Utikal, V. (2014) Affect and fairness: Dictator games under cognitive load. Journal of Economic Psychology 41:7787. doi: http://dx.doi.org/10.1016/j.joep.2012.08.007. [DA]

Schumpeter, J. A., Opie, R. \& Elliott, J. E. (1934) The theory of economic development: An inquiry into profits, capital, credit, interest, and the business cycle. Harvard University Press. [aPR]

Scofield, J., Gilpin, A. T., Pierucci, J. \& Morgan, R. (2013) Matters of accuracy and conventionality: Prior accuracy guides children's evaluations of others' actions. Developmental psychology 49(3):432. [SS]

Scott, W. R. (2001) Institutions and organizations: Ideas and interests, 2nd edition. Sage. [aPR]

Sell, A., Tooby, J. \& Cosmides, L. (2009) Formidability and the logic of human anger. Proceedings of the National Academy of Sciences USA 106:15073-78. doi:10.1073/pnas.0904312106. [MMK]

Seston, R. \& Kelemen, D. (2014) Children's conformity when acquiring novel conventions: The case of artifacts. Journal of Cognition and Development 15 (4):569-83. [SS]

Shalvi, S. \& De Dreu, C. K. W. (2014) Oxytocin promotes group serving dishonesty. Proceedings of the National Academy of Sciences USA 111:5503-507. [CKWDD]

Shariff, A. F., Norenzayan, A. \& Henrich, J. (2009) The birth of high gods: How the cultural evolution of supernatural policing influenced the emergence of complex, cooperative human societies, paving the way for civilization. In: Evolution, culture, and the human brain, ed. M. Schaller, A. Norenzayan, S. J. Heine, T. Yamagishi \& T. Kameda, pp. 119-36. Taylor and Francis. [aPR] 
References/Richerson et al.: Cultural group selection plays an essential role in explaining human cooperation

Shennan, S. (2001) Demography and cultural innovation: A model and its implications for the emergence of human culture. Cambridge Archaeological Journal 11(1):5-16. [aPR]

Shennan, S. J., Crema, E. R. \& Kerig, T. (2015) Isolation-by-distance, homophily, and "core" vs. "package" cultural evolution models in Neolithic Europe. Evolution and Human Behavior 36(2):103-19. doi: 10.1016/j.evolhumbehav.2014.09.006. [RMR]

Sheskin, M., Wynn, K. \& Bloom, P. (2014) Anti-equality: Social comparison in young children. Cognition 130(2):152-56. [JT, rPR]

Shtulman, A. \& Carey, S. (2007) Improbable or impossible? How children reason about the possibility of extraordinary events. Child Development 78(3):101532. $[\mathrm{SS}]$

Shutts, K., Kinzler, K. D., McKee, C. B. \& Spelke, E. S. (2009) Social information guides infants' selection of foods. Journal of Cognition and Development 10(12):1-17. [aPR]

Sigmund, K. (2007) Punish or perish? Retaliation and collaboration among humans. Trends in Ecology and Evolution 22(11):593-600. Available at: http://dx.doi.org/ 10.1016/j.tree.2007.06.012 [aPR]

Silva, A. S. \& Mace, R. (2014) Cooperation and conflict: Field experiments in Northern Ireland. Proceedings of the Royal Society B: Biological Sciences 281 (1792). (Published Online August 20, 2014). doi: 10.1098/rspb.2014.1435. Available at: http://rspb.royalsocietypublishing.org/content/281/1792/ 20141435 [RM, rPR]

Silva, A. S. \& Mace, R. (2015) Inter-group conflict and cooperation: Field experiments before, during and after sectarian riots in Northern Ireland. Frontiers in Psychology 6(1790). (Published Online November 6, 2015). doi: 10.3389/ fpsyg.2015.01790. Available at: http://journal.frontiersin.org/article/10.3389/ fpsyg.2015.01790/. [RM]

Simmons, M. (1979) History of Pueblo-Spanish relations to 1821. In: Handbook of North American Indians. Vol. 9: Southwest, ed. A. Ortiz, pp. 178-205. Smithsonian Institution. [rPR]

Simon, H. A. (1990) A mechanism for social selection and successful altruism Science 250(4988):1665-68. [aPR]

Singh, M. \& Boomsma, J. J. (2015) Policing and punishment across the domains of social evolution. Oikos 124(8): 971-82. doi:10.1111/oik.02064. [MS]

Smaldino, P. E. (2014) The cultural evolution of emergent group-level traits. Behavioral and Brain Sciences 37(3):243-95. doi: 10.1017/ S0140525X13001544. [rPR, DR, RMR]

Smaldino, P. E., Schank, J. C. \& McElreath, R. (2013) Increased costs of cooperation help cooperators in the long run. The American Naturalist 4:451-63. [rPR]

Smith, E. A. (2010) Communication and collective action: Language and the evolution of human cooperation. Evolution and Human Behavior 31(4):231-45. doi: 10.1016/j.evolhumbehav.2010.03.001. [aPR]

Snowdon, C. T. (2001) Social processes in communication and cognition in callitrichid monkeys: A review. Animal Cognition 4:247-57. [JMB]

Snowdon, C. T. (2013) Language parallels in New World primates. In: Animal models of speech and language disorders, ed. S. H. Helekar, pp. 241-61. Springer. [JMB]

Sobel, D. M. \& Kushnir, T. (2013) Knowledge matters: How children evaluate the reliability of testimony as a process of rational inference. Psychological Review 120(4):779-97. [SS]

Sobel, D. M. \& Macris, D. M. (2013) Children's understanding of speaker reliability between lexical and syntactic knowledge. Developmental Psychology 49(3):52332. $[\mathrm{SS}]$

Sober, E. \& Wilson, D. S. (1999) Unto others: The evolution and psychology of unselfish behavior. Harvard University Press. [OM]

Soltis, J., Boyd, R. \& Richerson, P. J. (1995) Can group-functional behaviors evolve by cultural group selection? An empirical test. Current Anthropology 36 (3):473-94. [arPR]

Sosis, R. \& Bressler, E. R. (2003) Cooperation and commune longevity: A test of the costly signaling theory of religion. Cross-Cultural Research 37:211-39. [aPR]

Sosis, R. \& Ruffle, B. (2003) Religious ritual and cooperation: Testing for a relationship on Israeli religious and secular Kibbutzim. Current Anthropology 44 (5):713-22. [aPR]

Spencer, B. \& Gillen, F. J. (1927) The Arunta: A study of a Stone Age people. Macmillan. [MS]

Spolaore, E. \& Wacziarg, R. (2013) How deep are the roots of economic development? Journal of Economic Literature 51(2):325-69. doi: 10.1257/ jel.51.2.325. $\quad[\mathrm{PH}]$

Stanford, C. B. (1999) The hunting apes: Meat eating and the origins of human behavior. Princeton University Press. [aPR]

Stark, R. (1997) The rise of Christianity: How the obscure, marginal Jesus movement became the dominant religious force in the western world in a few centuries. HarperCollins. [aPR]

Steffens, N. K., Haslam, S. A. \& Reicher, S. D. (2014) Up close and personal: Evidence that shared social identity is a basis for the "special" relationship that binds followers to leaders. The Leadership Quarterly 25(2):296-13. Available at: http://dx.doi.org/10.1016/j.leaqua.2013.08.008 [aPR]

Steinmo, S. (2010) The evolution of modern states: Sweden, Japan, and the United States. Cambridge University Press. [rPR]

Stephens, E. \& Koenig, M. (2015) Varieties of testimony: Children's selective learning in semantic and episodic domains. Cognition 137:182-88. [SS]

Stephens, E., Suárez, S. \& Koenig, M.A. (2015) Early testimonial learning: Monitoring speech acts and speakers. Advances in Child Development and Behavior 48:151-83. [SS]

Sterelny, K. (2012) The evolved apprentice: How evolution made humans unique. MIT Press. [rPR]

Sterelny, K. \& Hiscock, P. (2014) Symbols, signals, and the archaeological record. Biological Theory 9:1-3. [AF]

Steward, J. H. (1955) Theory of culture change: The methodology of multilinear evolution. University of Illinois Press. [aPR]

Stiller, J. \& Dunbar, R. I. M. (2007) Perspective-taking and memory capacity predict social network size. Social Networks 29:93-104. [CA-C]

Stiner, M. C. \& Kuhn, S. L. (2009) Paleolithic diet and the division of labor in Mediterranean Eurasia. In: Evolution of hominin diets: Integrating approaches to the study of Paleolithic subsistence, ed. J.-J. Hublin \& M. P. Richards, pp. 155-67. Springer Science+Business Media. [rPR]

Stivers, T., Enfield, N. J., Brown, P., Englert, C., Hayashi, M., Heinemann, T., Hoymann, G., Rossario, F., de Ruiter, J. P., Yoon, K.-E. \& Levinson, S. C. (2009) Universals and cultural variation in turn-taking in conversation. Proceedings of the National Academy of Sciences USA 106(26):10587-92. [JMB]

Stoeckel, L. E., Palley, L. S., Gollub, R. L., Niemi, S. M. \& Evins, A. E. (2014) Patterns of brain activation when mothers view their own child and dog: An fMRI study. PLoS One 9(10):e107205. [HH]

Stoll, D. (1990) Is Latin America turning Protestant? The politics of evangelical growth. University of California Press. [aPR]

Stout, D. (2012) Stone toolmaking and the evolution of human culture and cognition. In: Culture evolves, ed. A. Whitten, R. A. Hinde, C. B. Stringer \& K. N. Laland, pp. 197-14. Oxford University Press. [AF]

Strathearn, L. (2011) Maternal neglect: Oxytocin, dopamine and the neurobiology of attachment. Journal of Neuroendocrinology 23(11):1054-65. [JSC]

Suddendorf, T. (2013) The gap: The science of what separates us from other animals. Basic Books. [JSC]

Takahashi, D. Y., Narayanan, D. Z. \& Ghazanfar, A. A. (2013) Coupled oscillator dynamics of vocal turn-taking in monkeys. Current Biology 23(21):2162-68. [JMB]

Tanaka, Y. (1996) Social selection and the evolution of animal signals. Evolution 50:512-23. [RMN]

Tanis, M. \& Postmes, T. (2005) A social identity approach to trust: Interpersonal perception, group membership and trusting behavior. European Journal of Social Psychology 35:413-24. [CA-C]

Taylor, D. J. (2014) Evolution of the social contract. Doctoral dissertation, University of Bath, England. [DR]

Taylor, K. I. (1981) Knowledge and praxis in Sanumá food prohibitions. In: Working Papers on South American Indians: Food taboos in Lowland South America, vol. 3, ed. K. M. Kensinger \& W. H. Kracke. Bennington College. [MS]

Tennie, C., Call, J. \& Tomasello, M. (2009) Ratcheting up the ratchet: On the evolution of cumulative culture. Philosophical Transactions of the Royal Society B: Biological Sciences 364(1528):2405-15. doi: 10.1098/rstb.2009.0052. [aPR]

Tilly, C. (1992) Coercion, capital and European states: Wiley-Blackwell. [aPR]

Tishkoff, S. A., Reed, F. A., Ranciaro, A., Voight, B. F., Babbitt, C. C., Silverman, J. S., Powell, K., Mortensen, H., Hirbo, J.B., Osman, M., Ibrahim, M., Omar, S. A. Lema, G., Nyambo, T. B., Ghori, J., Bumpstead, S., Pritchard, J. K., Wray, G. A. \& Deloukas, P. (2007) Convergent adaptation of human lactase persistence in Africa and Europe. Nature Genetics 39(1):31-40. [aPR]

Tobias, J. A., Montgomerie, R. \& Lyon, B. E. (2012) The evolution of female ornaments and weaponry: Social selection, sexual selection and ecological competition. Philosophical Transactions of the Royal Society of London. Series B: Biological Sciences 367(1600):2274-93. doi: 10.1098/rstb.2011.0280. [RMN]

Tognetti, A., Berticat, C., Raymond, M. \& Faurie, C. (2012) Sexual selection of human cooperative behaviour: An experimental study in rural Senegal. PLoS ONE 7(9):e44403. [SL]

Tomasello, M. (1999) The cultural origins of human cognition. Harvard University Press. [aPR]

Tomasello, M. (2007) If they're so good at grammar, then why don't they talk? Hints from apes' and humans' use of gestures. Language Learning and Development 3 (2):133-56. [JMB]

Tomasello, M. (2008) Origins of human communication. MIT Press. [HG]

Tomasello, M. (2014) A natural history of human thinking. Harvard University Press. [HG]

Tomasello, M. \& Carpenter, M. (2007) Shared intentionality. Developmental Science 10(1):121-25. doi: 10.1111/j.1467-7687.2007.00573.x. [aPR] 
Tomasello, M., Carpenter, M., Call, J., Behne, T. \& Moll, H. (2005) Understanding and sharing intentions: The origins of cultural cognition. Behavioral and Brain Sciences 28(5):675-91. doi: 10.1017/S0140525X05000129. [aPR, JSC]

Tomasello, M., Melis, A. P., Tennie, C., Wyman, E. \& Herrmann, E. (2012) Two key steps in the evolution of human cooperation: The interdependence hypothesis. Current Anthropology 53(6):673-92. doi: 10.1086/668207. [aPR]

Tooby, J. (2014) What scientific idea is ready for retirement? Learning and culture Edge. (Online publication). Available at: http://edge.org/responses/what-scientific-idea-is-ready-for-retirement [aPR]

Tooby, J. \& Cosmides, L. (1989) Evolutionary psychology and the generation of culture, 1. Theoretical considerations. Ethology and Sociobiology 10(1-3):2949. [arPR, MMK]

Tooby, J. \& Cosmides, L. (1992) The psychological foundations of culture. In: The adapted mind: Evolutionary psychology and the generation of culture, ed. J. Barkow, L. Cosmides \& J. Tooby, pp. 19-136. Oxford University Press. [arPR, MMK]

Tooby, J. \& Cosmides, L. (2010) Groups in mind: The coalitional roots of war and morality. In: Human morality and sociality: Evolutionary and comparative perspectives, ed. H. Hogh-Olesen, pp. 91-234. Palgrave Macmillan. [JT]

Tooby, J. \& DeVore, I. (1987) The reconstruction of hominid behavioral evolution through strategic modeling. In: The evolution of human behavior: Primate models, ed. W. G. Kinzey, pp. 183-237. State University of New York Press. [MMK, JT]

Tostevin, G. B. (2013) Seeing lithics: A middle-range theory for testing cultural transmission in the Pleistocene. Harvard University American School of Prehistoric Research and Oxbow Books. [aPR]

Trevarthen, C. (2007) The intersubjective psychobiology of human meaning: Learning of culture depends on interest for co-operative practical work - and affection for the joyful art of good company. Psychoanalytic Dialogues: The International Journal of Relational Perspectives 19(5):507-18. [JSC]

Trevarthen, C. (2011) What is it like to be a person who knows nothing? Defining the active intersubjective mind of a newborn human being. Infant and Child Development 20(1):119-35. [JSC]

Tronick, E. Z. (2007) The neurobehavioral and social-emotional development of infants and children. W. W. Norton. [JSC]

Trut, L. (1999) Early canid domestication: The farm-fox experiment. American Scientist 87:160-69. [JSC]

Turchin, P. (2003) Historical dynamics. Princeton University Press. [aPR]

Turchin, P. (2006) War and peace and war: The life cycles of imperial nations. Pi Press. [arPR]

Turchin, P. (2008) Arise “cliodynamics." Nature 454(7200):34-35. doi 10.1038/ 454034a. [aPR]

Turchin, P. (2009) A theory for the formation of large empires. Journal of Global History 4:191-217. [arPR]

Turchin, P., Brennan, R., Currie, T., Feeney, K., Francois, P., Hoyer, D., Manning J., Marciniak, A., Mullins, D., Palmisano, A., Peregrine, P., Turner, E. A. L., Whitehouse, H. (2015). Seshat: The Global History Databank. Cliodynamics 6:77-107. [PT]

Turchin, P., Currie, T. E., Turner, E. A. L. \& Gavrilets, S. (2013) War, space, and the evolution of Old World complex societies. Proceedings of the National Academy of Sciences USA 110:16384-89. [PT]

Turchin, P. \& Nefedov, S. A. (2009) Secular cycles. Princeton University Press. [arPR]

Turchin, P., Whitehouse, H., Francois, P., Slingerland, E. \& Collard, M. (2012) A historical database of sociocultural evolution. Cliodynamics: The Journal of Theoretical and Mathematical History 3(2):271-93. [aPR]

van Schaik, C. P. \& Burkart, J. M. (2010) Mind the gap: Cooperative breeding and the evolution of our unique features. In: Mind the gap: Tracing the origins of human universals, ed. P. M. Kappeler \& J. Silk, pp. 477-96. Springer. [JMB]

Veblen, T. (1898) Why is economics not an evolutionary science? The Quarterly Journal of Economics 12(4):373-97. [aPR]

Vigil, J. M. (2009) A socio-relational framework of sex differences in the expression of emotion. Behavioral and Brain Sciences 32(5):375-428. [JMV]

Vigil, J. M. \& Alcock, J. (2014) Tough guys or cry babies? Disentangling the role of examiner gender on patient pain reports. Pain Research and Management 19: e9-e12. [JMV]

Vigil, J. M. \& Coulombe, P. (2011) Biological sex and audience affects pain intensity and observational coding of other people's pain behaviors. Pain 152:2125-30. [JMV]

Vigil, J. M., Pendleton, P., Coulombe, P, Vowels, K., Alcock, J. \& Smith, B. (2014a) Pain patients and who they live with: A correlational study of co-residence patterns and pain interference. Pain Research and Management 19:el09el14. [JMV]

Vigil, J. M., Rowell, L. N., Alcock, J. \& Maestes, R. (2014b) Laboratory personnel gender and cold pressor apparatus affect subjective pain reports. Pain Research and Management 19:e13-e18. [JMV]

Vigil, J. M., Rowell, N., Chouteau, S., Chavez, A., Jaramillo, E., Neal, M. \& Waid, D. (2013) Sex differences in how social networks and relationship quality influence experimental pain sensitivity. PLoS ONE 8:e78663. doi:10.1371/journal. pone.0078663. [JMV]

Vigil, J. M. \& Strenth, C. (2014) No pain, no social gains: A social-signaling perspective of human pain behaviors. World Journal of Anesthesiology 3(1):18-30. Available at: http://www.wjgnet.com/2218-6182/abstract/v3/i1/18.htm. [JMV]

Vigil, J. M., Strenth, C, Trujillo, T. \& Gangestad, S. W. (2014c) Fluctuating experimental pain sensitivities across the menstrual cycle are contingent on women's current romantic relationship status. PLoS ONE 9:e91993. doi:10.1371/journal pone.0091993. [JMV]

Vigil, J. M., Torres, D., Wolff, A. \& Hughes, K. (2014d) Exposure to virtual social stimuli modulates subjective pain reports. Pain Research and Management 19: el03-e108. [JMV]

Vitale, A., Zanzoni, M., Queyras, A. \& Chiarotti, F. (2003) Degree of social contact affects the emission of food calls in the common marmoset (Callithrix jacchus). American Journal of Primatology 59:21-28. [JMB]

Walker, J. M., Gardner, R., Herr, A. \& Ostrom, E. (2000) Collective choice in the commons: Experimental results on proposed allocation rules and votes. The Economic Journal 110(460):212-34. [DA]

Walker, R. S., Wichmann, S., Mailund, T. \& Atkisson, C. J. (2012) Cultural phylogenetics of the Tupi language family in lowland South America. PLOS ONE 7(4): e35025. doi: 10.1371/journal.pone.0035025. [aPR]

Warneken, F. \& Tomasello, M. (2009) Varieties of altruism in children and chimpanzees. Trends in Cognitive Sciences 13(9):397-402. [aPR]

Washburn, S. L. (1959) Speculations on the interrelations of the history of tools and biological evolution. Human Biology 31(1):21-31. [aPR]

Watanabe, J. M. (1990) From saints to shibboleths: Image, structure, and identity in Maya religious syncretism. American Ethnologist 17(1):131-50. [CSa]

Wehmeier, S. \& Hornby, A. S. (2000) Oxford advanced learner's dictionary of current English. Cornelsen \& Oxford University Press. [CSi]

Weingarten, C. \& Chisholm, J. (2009) Attachment and cooperation in religious groups: An example of a mechanism for cultural group selection. Current Anthropology 50(6):759-85. [JSC]

West, R. (1941) Black lamb and grey falcon. Penguin. [aPR]

West, S. A., El Mouden, C. \& Gardner, A. (2011) Sixteen common misconceptions about the evolution of cooperation in humans. Evolution and Human Behavior 32(4):231-62. doi: 10.1016/j.evolhumbehav.2010.08.001. [aPR, PB]

West, S. A., Griffin, A. S. \& Gardner, A. (2007) Social semantics: Altruism, cooperation, mutualism, strong reciprocity and group selection. Journal of Evolutionary Biology 20(2):415-32. doi: 10.1111/j.1420-9101.2006.01258.x. [aPR, RMN]

West-Eberhard, M. J. (1979) Sexual selection, social competition, and evolution Proceedings of the American Philosophical Society 123(4):222-34. [RMN]
[R

West-Eberhard, M. J. (1983) Sexual selection, social competition, and speciation. Quarterly Review of Biology 58(2):155-83. [RMN]

Westgarth, C., Heron, J., Ness, A. R., Bundred, P., Gaskell, R. M., Coyne, K. P., German, A. J., McCune, S. \& Dawson, S. (2010) Family pet ownership during childhood: Findings from a UK birth cohort and implications for public health research. International Journal of Environmental Research and Public Health $7(10): 3704-29 . \quad[\mathrm{HH}]$

White, L. (1959) The evolution of culture. McGrew-Hill. [DR]

Whitehead, H. \& Rendell, L. (2015) The cultural lives of whales and dolphins. University of Chicago Press. [rPR]

Whitehouse, H. (2002) Modes of religiosity: Towards a cognitive explanation of the sociopolitical dynamics of religion. Method and Theory in the Study of Religion 14(3/4):293-315. [aPR]

Whiten, A. \& Erdal, D. (2012) The human socio-cognitive niche and its evolutionary origins. Philosophical Transactions of the Royal Society, B 367:2119-29. [AF, AW]

Whiten, A., Goodall, J., McGrew, W. C., Nishida, T., Reynolds, V., Sugiyama, Y., Tutin, C. E. G., Wrangham, R. W. \& Boesch, C. (1999) Cultures in chimpanzees. Nature 399:682-85. [rPR]

Whiten, A., McGuigan, N., Marshall-Pescini, S. \& Hopper, L. M. (2009) Emulation, imitation, over-imitation and the scope of culture for child and chimpanzee. Philosophical Transactions of the Royal Society B 364:2417-28. [arPR]

Wierzbicka, A. (1992) Semantics, culture, and cognition: Human concepts in culturespecific configurations. Oxford University Press. [aPR]

Wiessner, P. (2002) The vines of complexity: Egalitarian structures and the institutionalization of inequality among the Enga. Current Anthropology 43(2):23369. [MS]

Wilkins, A. S., Wrangham, R. W. \& Tecumseh Fitch, W. (2014) The "domestication syndrome" in mammals: A unified explanation based on neural crest cell behavior and genetics. Genetics 197(3):795-808. [HG]

Wilkins, J., Schoville, B. J., Brown, K. S. \& Chazan, M. (2012) Evidence for early hafted hunting technology. Science 338:942-46. [HG]

Wilks, M., Collier-Baker, E. \& Nielsen, M. (2015) Preschool children favor copying a successful individual over an unsuccessful group. Developmental Science 18 (6):1014-24. doi: 10.1111/desc.12274. [SS]

Williams, G. C. (1966) Adaptation and natural selection. Princeton University Press. [PB, MMK] 
References/Richerson et al.: Cultural group selection plays an essential role in explaining human cooperation

Williams, G. C. (1992) Natural selection: Domains, levels and challenges: Oxford University Press. [rPR]

Wilson, D. S. (2002) Darwin's cathedral: Evolution, religion, and the nature of society. University of Chicago Press. [aPR]

Wilson, D. S. \& Kniffin, K. M. (1999) Multilevel selection and the social transmission of behavior. Human Nature 10(3):291-310. [aPR]

Wilson, E. O. (2012) The social conquest of earth. W. W. Norton. [HG, aPR]

Wolf, J. B., Brodie, E. D., III \& Moore, A. J. (1999) Interacting phenotypes and the evolutionary process. II. Selection resulting from social interactions. American Naturalist 153:254-66. [RMN]

Wolfe, N. D., Dunavan, C. P. \& Diamond, J. (2007) Origins of major human infectious diseases. Nature 447(7142):279-83. [aPR]

Woolley, J. D. \& E Ghossainy, M. (2013) Revisiting the fantasy-reality distinction: Children as naïve skeptics. Child Development 84(5):1496-510. [SS]

Wrangham, R. (2009) Catching fire: How cooking made us human. Basic Books. [aPR]

Wrangham, R. W. \& Carmody, R. (2010) Human adaptation to the control of fire. Evolutionary Anthropology 19:187-99. [HG]

Wright, S. (1943) Isolation by distance. Genetics 28:114-38. [RMR]
Yamagishi, T., Hashimoto, H. \& Schug, J. (2008) Preferences versus strategies as explanations for culture-specific behavior. Psychological Science 19:579-84. [PB]

Yamagishi, T., Jin, N. \& Kiyonari, T. (1999) Bounded generalized reciprocity: Ingroup boasting and ingroup favoritism. Advances in Group Processes 16:161-97. [CKWDD]

Zefferman, M. R. (2014a) Direct reciprocity under uncertainty does not explain oneshot cooperation, but demonstrates the benefits of a norm psychology. Evolution and Human Behavior 35:358-67. doi: 10.1016/j.evolhumbehav.2014.04.003. [arPR, JH]

Zefferman, M. R. (2014b) When repentance and forgiveness are possible, direct reciprocity does not explain one-shot generosity, even under arbitrarily high levels of uncertainty. Evolution and Human Behavior 35(6):548-49. Available at: http://dx.doi.org/10.1016/j.evolhumbehav.2014.07.003 [rPR]

Zhou, W. X., Sornette, D., Hill, R. A. \& Dunbar, R. I. M. (2005) Discrete hierarchical organization of social group sizes. Proceedings of the Royal Society $B$ 272:439-44. [CA-C]

Zuberbühler, K. (2012) Cooperative breeding and the evolution of vocal flexibility. In: The Oxford handbook of language evolution, ed. M. Tallerman \& K. Gibson, pp. 71-81. Oxford University Press. [JMB] 